\title{
Ligand-Accelerated Iron Photocatalysis Enabling Decarboxylative Alkylation of Heteroarenes
}

\author{
Zhenlong Li, Xiaofei Wang, Siqi Xia, and Jian Jin*
}

\section{Table of Contents}

I. General Information

II. Preliminary Mechanistic Studies

III. Reaction Setup

IV. Experimental Procedures and Product Characterization

V. NMR Spectra 


\section{General Information}

Commercial reagents, lepidine, isobutyric acid, $\mathrm{FeSO}_{4} \cdot 7 \mathrm{H}_{2} \mathrm{O}$, picolinic acid, $\mathrm{NaBrO}_{3}$, water and DMSO were purchased from J\&K, TCI, Acros, Sigma-Aldrich and Fisher Chemical, and used directly without purification. All heteroarenes, carboxylic acids and other reagents were utilized directly from commercial suppliers. Organic solutions were concentrated under reduced pressure on a Büchi rotary evaporator with a recirculating cooling system. Chromatographic purification of products was accomplished by flash chromatography on silica gel (Santai, 230-400 mesh). Thin layer chromatography (TLC) was performed on Huanghai $0.4-0.5 \mathrm{~mm}$ silica gel plates. Visualization of the developed chromatogram was performed by fluorescence quenching. ${ }^{1} \mathrm{H}$ and ${ }^{13} \mathrm{C}$ NMR spectra were recorded on a Bruker UltraShield Plus $400 \mathrm{MHz}(101 \mathrm{MHz})$ instrument, and are internally referenced to residual protio solvent signals (note: $\mathrm{CDCl}_{3}$ referenced at 7.26 and $77.0 \mathrm{ppm}$ respectively). Data for ${ }^{1} \mathrm{H}$ NMR are reported as follows: chemical shift ( $\delta$ ppm), multiplicity $(\mathrm{s}=$ singlet, $\mathrm{d}=$ doublet, $\mathrm{t}=$ triplet, $\mathrm{q}=$ quartet, $\mathrm{m}=$ multiplet, $\mathrm{dd}=$ doublet of doublets, $\mathrm{dt}=$ doublet of triplets, $\mathrm{br}=$ broad $)$, coupling constant $(\mathrm{Hz})$ and integration. Data for ${ }^{13} \mathrm{C}$ NMR are reported in terms of chemical shift and no special nomenclature is used for equivalent carbons. IR spectra were recorded on a Perkin Elmer Spectrum 100 FTIR spectrometer and are reported in wavenumbers $\left(\mathrm{cm}^{-1}\right)$. High resolution mass spectra were obtained at National Center for Organic Mass Spectrometry in Shanghai Institute of Organic Chemistry, Chinese Academy of Sciences on a Thermo Fisher Scientific LTQ FTICR-MS instrument with electrospray ionization method. 


\section{Preliminary Mechanistic Studies}

A Radical quenching experiments

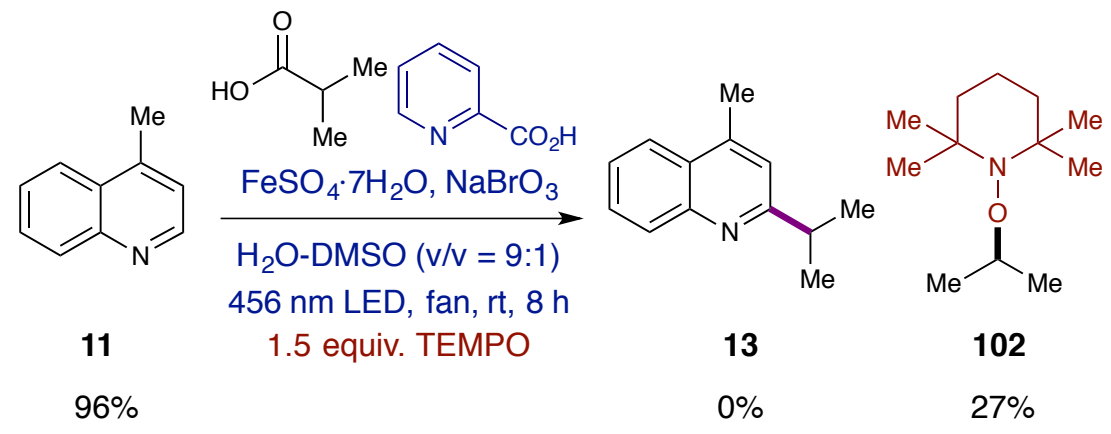

B Light/dark experiments

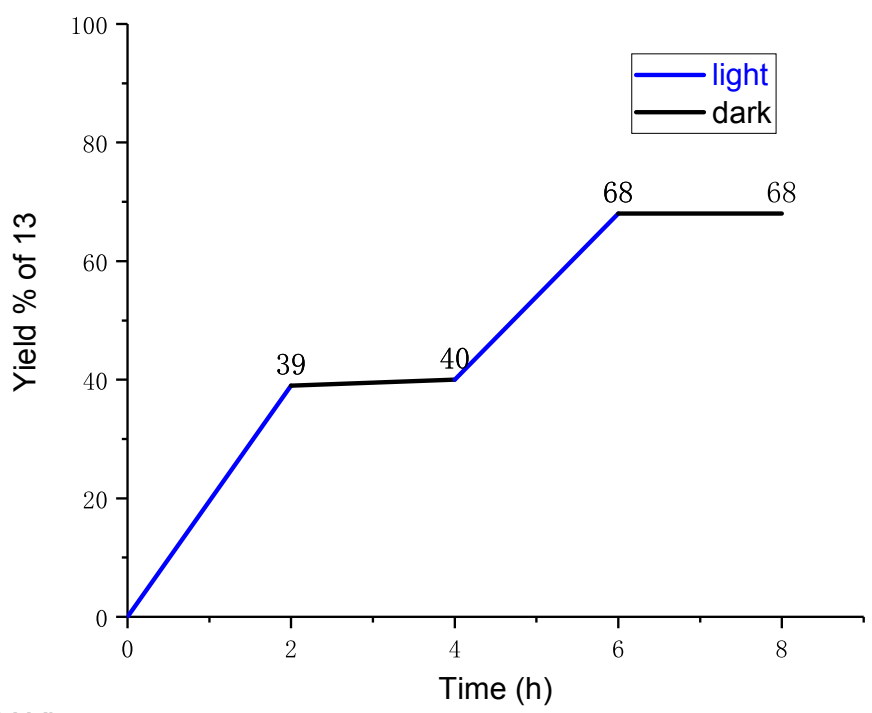

C UV-Vis spectra

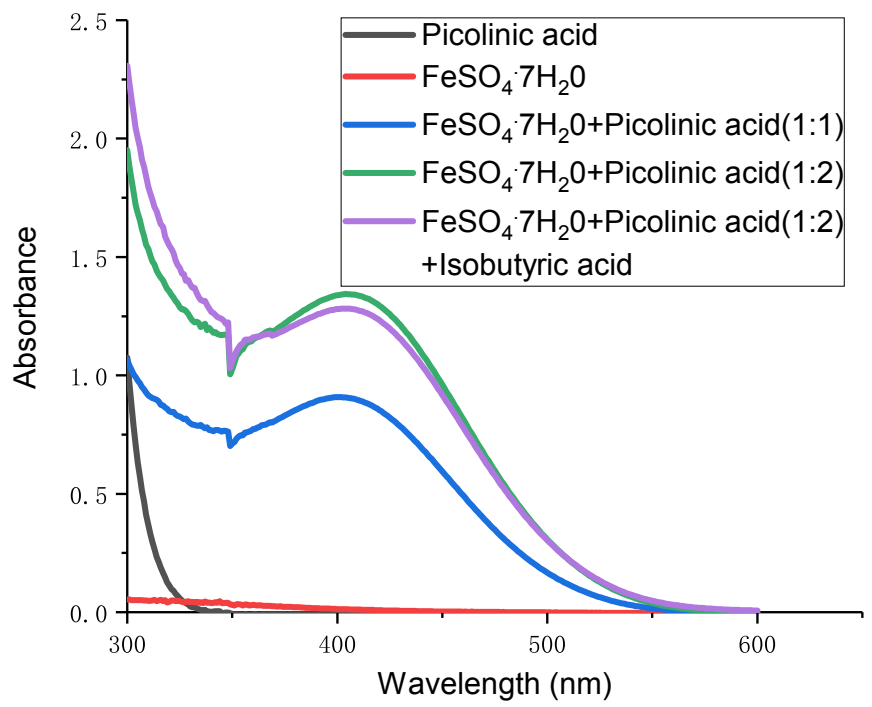

Figure S1. Preliminary mechanistic studies. 


\begin{tabular}{|c|c|c|c|c|c|}
\hline \multicolumn{2}{|c|}{$0.5 \mathrm{mmol} 11$} & O equiv. 12 & $\begin{array}{l}\mathrm{FeSO}_{4} \cdot 7 \mathrm{H}_{2} \mathrm{O}, \text { oxida } \\
\mathrm{H}_{2} \mathrm{O}-\mathrm{DMSO}(\mathrm{v} / \mathrm{v}=9 \\
40 \mathrm{~W} \text { LED, fan, rt, } 8\end{array}$ & $\begin{array}{l}\longrightarrow \\
\mathrm{h}\end{array}$ & 3 \\
\hline ntry & iron (II) & picolinic acid & oxidant & wavelength & yield [\%] \\
\hline $1^{b}$ & - & - & - & $456 \mathrm{~nm}$ & 40 \\
\hline $2^{c}$ & - & - & 2 equiv. $\mathrm{NaBrO}_{3}$ & $456 \mathrm{~nm}$ & 0 \\
\hline 3 & $5 \mathrm{~mol} \%$ & - & 2 equiv. $\mathrm{NaBrO}_{3}$ & $456 \mathrm{~nm}$ & 0 \\
\hline 4 & $5 \mathrm{~mol} \%$ & $5 \mathrm{~mol} \%$ & 2 equiv. $\mathrm{NaBrO}_{3}$ & $456 \mathrm{~nm}$ & 60 \\
\hline 5 & $5 \mathrm{~mol} \%$ & $10 \mathrm{~mol} \%$ & 2 equiv. $\mathrm{NaBrO}_{3}$ & $456 \mathrm{~nm}$ & 94 \\
\hline 6 & $5 \mathrm{~mol} \%$ & $15 \mathrm{~mol} \%$ & 2 equiv. $\mathrm{NaBrO}_{3}$ & $456 \mathrm{~nm}$ & 85 \\
\hline 7 & $5 \mathrm{~mol} \%$ & $10 \mathrm{~mol} \%$ & 2 equiv. $\mathrm{NaBrO}_{3}$ & $440 \mathrm{~nm}$ & 85 \\
\hline 8 & $5 \mathrm{~mol} \%$ & $10 \mathrm{~mol} \%$ & 2 equiv. $\mathrm{NaBrO}_{3}$ & $427 \mathrm{~nm}$ & 83 \\
\hline 9 & $5 \mathrm{~mol} \%$ & $10 \mathrm{~mol} \%$ & 2 equiv. $\mathrm{NaBrO}_{3}$ & $390 \mathrm{~nm}$ & 49 \\
\hline 10 & $5 \mathrm{~mol} \%$ & $10 \mathrm{~mol} \%$ & 2 equiv. $\mathrm{NaClO}_{3}$ & $456 \mathrm{~nm}$ & 39 \\
\hline 11 & $10 \mathrm{~mol} \%$ & $20 \mathrm{~mol} \%$ & 3 equiv. $\mathrm{NaClO}_{3}$ & $440 \mathrm{~nm}$ & 90 \\
\hline 12 & $10 \mathrm{~mol} \%$ & $20 \mathrm{~mol} \%$ & 3 equiv. $\mathrm{NaIO}_{4}$ & $440 \mathrm{~nm}$ & 80 \\
\hline 13 & $10 \mathrm{~mol} \%$ & $20 \mathrm{~mol} \%$ & 3 equiv. $\mathrm{KIO}_{3}$ & $440 \mathrm{~nm}$ & 86 \\
\hline 14 & - & $10 \mathrm{~mol} \%$ & 2 equiv. $\mathrm{NaBrO}_{3}$ & $456 \mathrm{~nm}$ & 0 \\
\hline $15^{b}$ & - & - & - & dark & 0 \\
\hline 16 & $5 \mathrm{~mol} \%$ & $10 \mathrm{~mol} \%$ & 2 equiv. $\mathrm{NaBrO}_{3}$ & dark & 0 \\
\hline 17 & $5 \mathrm{~mol} \%$ & $10 \mathrm{~mol} \%$ & 2 equiv. $\mathrm{NaBrO}_{3}$ & dark, $80^{\circ} \mathrm{C}$ & 0 \\
\hline
\end{tabular}

\section{Figure S2. Control experiments.}




\section{Reaction Setup}

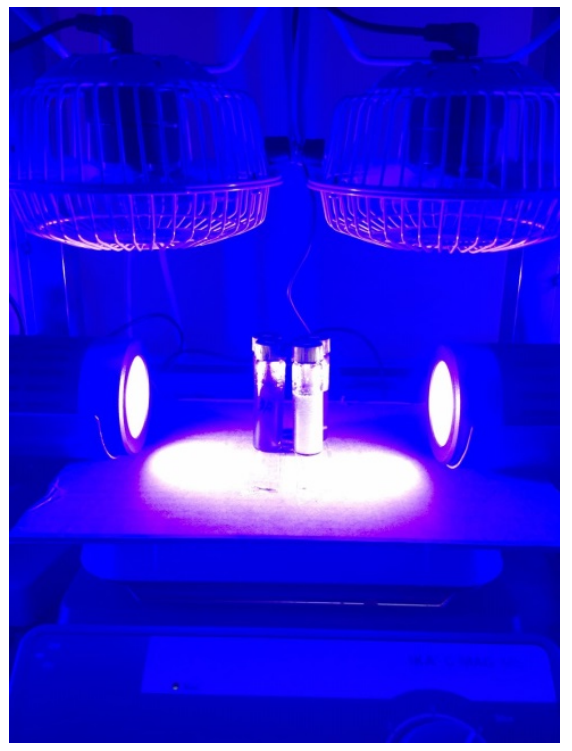

Figure S3. Reaction setup with a magnetic stirrer, two Kessil 40 W 456 nm LED lamps, two mini fans and four reaction vials. 


\section{Experimental Procedures and Product Characterization}

General Procedure A for the Decarboxylative Alkylation: To an $8 \mathrm{~mL}$ vial equipped with a Teflon septum and a magnetic stir bar was charged $\mathrm{FeSO}_{4} \cdot 7 \mathrm{H}_{2} \mathrm{O}(7.0 \mathrm{mg}, 0.025$ mmol, 0.05 equiv.), picolinic acid (6.2 $\mathrm{mg}, 0.05 \mathrm{mmol}, 0.1$ equiv.), heteroarene (0.50 mmol, 1.0 equiv.), carboxylic acid (5.0 mmol, 10 equiv.), $\mathrm{NaBrO}_{3}(150.9 \mathrm{mg}, 1.0 \mathrm{mmol}$, 2.0 equiv.), $4.5 \mathrm{~mL}$ of $\mathrm{H}_{2} \mathrm{O}$ and $0.5 \mathrm{~mL}$ of DMSO. The reaction mixture was degassed by sparging with nitrogen for 10 min with an outlet needle, and then irradiated with Kessil $40 \mathrm{~W} 456 \mathrm{~nm}$ LEDs (approximately $8 \mathrm{~cm}$ away from the light source) under two mini fans at room temperature. Upon reaction completion as judged by TLC and LCMS (8-24 hours), the reaction mixture was diluted with $1 \mathrm{M} \mathrm{NaOH}$ aqueous solution $(10 \mathrm{~mL})$, extracted with $\mathrm{CH}_{2} \mathrm{Cl}_{2}(3 \times 30 \mathrm{~mL})$, dried over $\mathrm{Na}_{2} \mathrm{SO}_{4}$, and concentrated in vacuo. Purification of the crude product by flash chromatography on silica gel using the indicated solvent system afforded the desired product.

General Procedure B for the Decarboxylative Alkylation: To an $8 \mathrm{~mL}$ vial equipped with a Teflon septum and a magnetic stir bar was charged $\mathrm{FeSO}_{4} \cdot 7 \mathrm{H}_{2} \mathrm{O}(14.0 \mathrm{mg}, 0.05$ mmol, 0.1 equiv.), picolinic acid (12.4 mg, $0.1 \mathrm{mmol}, 0.2$ equiv.), heteroarene (0.50 mmol, 1.0 equiv.), carboxylic acid (5.0 mmol, 10 equiv.), $\mathrm{NaClO}_{3}(161.3 \mathrm{mg}, 1.5 \mathrm{mmol}$, 3.0 equiv.), $4.5 \mathrm{~mL}$ of $\mathrm{H}_{2} \mathrm{O}$ and $0.5 \mathrm{~mL}$ of DMSO. The reaction mixture was degassed by sparging with nitrogen for 10 min with an outlet needle, and then irradiated with Kessil $40 \mathrm{~W} 440 \mathrm{~nm}$ LEDs (approximately $8 \mathrm{~cm}$ away from the light source) under two mini fans at room temperature. Upon reaction completion as judged by TLC and LCMS (8-36 hours), the reaction mixture was diluted with $1 \mathrm{M} \mathrm{NaOH}$ aqueous solution $(10 \mathrm{~mL})$, extracted with $\mathrm{CH}_{2} \mathrm{Cl}_{2}(3 \times 30 \mathrm{~mL})$, dried over $\mathrm{Na}_{2} \mathrm{SO}_{4}$, and concentrated in vacuo. 
Purification of the crude product by flash chromatography on silica gel using the indicated solvent system afforded the desired product.

Representative example at 2 mmol scale: To a $40 \mathrm{~mL}$ vial equipped with a Teflon septum and a magnetic stir bar was charged $\mathrm{FeSO}_{4} \cdot 7 \mathrm{H}_{2} \mathrm{O}(28.0 \mathrm{mg}, 0.1 \mathrm{mmol}, 0.05$ equiv.), picolinic acid (24.8 mg, $0.2 \mathrm{mmol}, 0.1$ equiv.), lepidine (289.2 mg, $2.0 \mathrm{mmol}, 1.0$ equiv.), isobutyric acid (1.78 g, $20.0 \mathrm{mmol}, 10$ equiv.), $\mathrm{NaBrO}_{3}$ (603.6 mg, $4.0 \mathrm{mmol}, 2.0$ equiv.), $18 \mathrm{~mL}$ of $\mathrm{H}_{2} \mathrm{O}$ and $2 \mathrm{~mL}$ of DMSO. The reaction mixture was degassed by sparging with nitrogen for 10 min with an outlet needle, and then irradiated with Kessil $40 \mathrm{~W} 456 \mathrm{~nm}$ LEDs (approximately $8 \mathrm{~cm}$ away from the light source) under two mini fans at room temperature. After $8 \mathrm{~h}$, the reaction mixture was diluted with $1 \mathrm{M} \mathrm{NaOH}$ aqueous solution $(40 \mathrm{~mL})$, extracted with $\mathrm{CH}_{2} \mathrm{Cl}_{2}(3 \times 120 \mathrm{~mL})$, dried over $\mathrm{Na}_{2} \mathrm{SO}_{4}$, and concentrated in vacuo. Purification of the crude product by flash chromatography $(10 \%$ ethyl acetate/hexanes) on silica gel provided the desired product 2-isopropyl-4methylquinoline as a colorless oil (320.6 mg, 87\% yield).

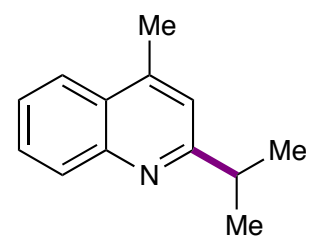

13

2-Isopropyl-4-methylquinoline (13): According to the general procedure A, $\mathrm{FeSO}_{4} \cdot 7 \mathrm{H}_{2} \mathrm{O}(7.0 \mathrm{mg}, 0.025 \mathrm{mmol}, 0.05$ equiv.), picolinic acid $(6.2 \mathrm{mg}, 0.05 \mathrm{mmol}, 0.1$ equiv.), lepidine (72.3 mg, $0.50 \mathrm{mmol}, 1.0$ equiv.), isobutyric acid (445.0 mg, $5.0 \mathrm{mmol}$, 10 equiv.), $\mathrm{NaBrO}_{3}$ (150.9 mg, $1.0 \mathrm{mmol}, 2.0$ equiv.), $4.5 \mathrm{~mL}$ of $\mathrm{H}_{2} \mathrm{O}$ and $0.5 \mathrm{~mL}$ of DMSO, $456 \mathrm{~nm}$ LEDs were used. After 8 hours, the reaction mixture was subjected to the workup procedure outlined in the general procedure and purified by flash 
chromatography ( $10 \%$ ethyl acetate/hexanes) to provide the title compound as a colorless oil (87.1 mg, 94\% yield). ${ }^{1} \mathrm{H}$ NMR (400 MHz, $\left.\mathrm{CDCl}_{3}\right) \delta 8.07$ (d, $\left.J=8.4 \mathrm{~Hz}, 1 \mathrm{H}\right), 7.94$ (d, $J=8.3 \mathrm{~Hz}, 1 \mathrm{H}), 7.67(\mathrm{t}, J=7.6 \mathrm{~Hz}, 1 \mathrm{H}), 7.50(\mathrm{t}, J=7.5 \mathrm{~Hz}, 1 \mathrm{H}), 7.18(\mathrm{~s}, 1 \mathrm{H}), 3.23$ (hept, $J=7.0 \mathrm{~Hz}, 1 \mathrm{H}), 2.69(\mathrm{~s}, 3 \mathrm{H}), 1.39(\mathrm{~d}, J=7.0 \mathrm{~Hz}, 6 \mathrm{H}) ;{ }^{13} \mathrm{C} \mathrm{NMR}\left(101 \mathrm{MHz}, \mathrm{CDCl}_{3}\right) \delta$ $167.28,147.40,144.41,129.36,128.94,126.95,125.38,123.51,119.69,37.14,22.48$, 18.80; HRMS (ESI) $\mathrm{m} / \mathrm{z}$ calculated for $\mathrm{C}_{13} \mathrm{H}_{16} \mathrm{~N}\left[(\mathrm{M}+\mathrm{H})^{+}\right]$186.1277, found 186.1276; IR (film) 2960, 2925, 2868, 1603, 1507, 1448, 1411, 1088, 948, 862, $755 \mathrm{~cm}^{-1}$. Spectra data are consistent with those reported in the literature: J. Am. Chen. Soc. 2017, 139, 1431514321.

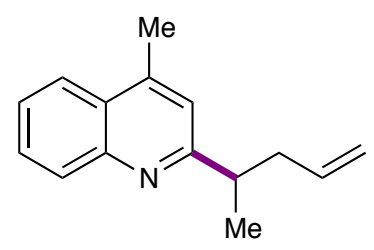

14

4-Methyl-2-(pent-4-en-2-yl)quinoline (14): According to the general procedure B, $\mathrm{FeSO}_{4} \cdot 7 \mathrm{H}_{2} \mathrm{O}$ (14.0 mg, $0.05 \mathrm{mmol}, 0.1$ equiv.), picolinic acid (12.4 mg, $0.1 \mathrm{mmol}, 0.2$ equiv.), lepidine (72.3 mg, $0.50 \mathrm{mmol}, 1.0$ equiv.), 2-methylpent-4-enoic acid (582.3 mg, 5.0 mmol, 10 equiv.), $\mathrm{NaClO}_{3}$ (161.3 mg, $1.5 \mathrm{mmol}, 3.0$ equiv.), $4.0 \mathrm{~mL}$ of $\mathrm{H}_{2} \mathrm{O}$ and 1.0 $\mathrm{mL}$ of DMSO, $440 \mathrm{~nm}$ LEDs were used. After 24 hours, the reaction mixture was subjected to the workup procedure outlined in the general procedure and purified by flash chromatography ( $5 \%$ ethyl acetate/hexanes) to provide the title compound as a colorless oil (65.4 mg, 62\% yield). ${ }^{1} \mathrm{H}$ NMR (400 MHz, $\left.\mathrm{CDCl}_{3}\right) \delta 8.06(\mathrm{~d}, J=8.1 \mathrm{~Hz}, 1 \mathrm{H}), 7.94(\mathrm{~d}$, $J=8.3 \mathrm{~Hz}, 1 \mathrm{H}), 7.67(\mathrm{ddd}, J=8.4,6.8,1.4 \mathrm{~Hz}, 1 \mathrm{H}), 7.50(\mathrm{ddd}, J=8.2,6.8,1.3 \mathrm{~Hz}, 1 \mathrm{H})$, $7.14(\mathrm{~s}, 1 \mathrm{H}), 5.87-5.71(\mathrm{~m}, 1 \mathrm{H}), 5.05(\mathrm{dd}, J=17.1,1.8 \mathrm{~Hz}, 1 \mathrm{H}), 5.00-4.91(\mathrm{~m}, 1 \mathrm{H})$, $3.14(\mathrm{~h}, J=7.1 \mathrm{~Hz}, 1 \mathrm{H}), 2.68(\mathrm{~s}, 3 \mathrm{H}), 2.62(\mathrm{dt}, J=13.7,6.9 \mathrm{~Hz}, 1 \mathrm{H}), 2.42(\mathrm{dt}, J=14.2$, 
$7.7 \mathrm{~Hz}, 1 \mathrm{H}), 1.37(\mathrm{~d}, J=7.0 \mathrm{~Hz}, 3 \mathrm{H}) ;{ }^{13} \mathrm{C} \mathrm{NMR}\left(101 \mathrm{MHz}, \mathrm{CDCl}_{3}\right) \delta 165.86,147.47$, $144.37,136.82,129.40,128.96,127.00,125.46,123.54,120.34,116.15,42.40,41.06$, 20.09, 18.82; HRMS (ESI) $\mathrm{m} / \mathrm{z}$ calculated for $\mathrm{C}_{15} \mathrm{H}_{18} \mathrm{~N}\left[(\mathrm{M}+\mathrm{H})^{+}\right] 212.1434$, found 212.1433; IR (film) 2960, 2924, 1602, 1560, 1507, 1448, 991, 911, 872, 862, $786 \mathrm{~cm}^{-1}$.

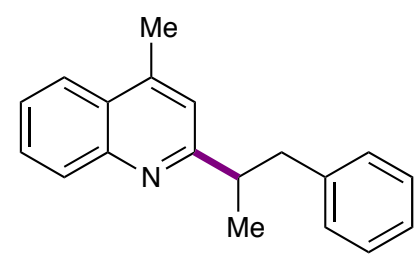

15

4-Methyl-2-(1-phenylpropan-2-yl)quinoline (15): According to the general procedure $\mathrm{A}, \mathrm{FeSO}_{4} \cdot 7 \mathrm{H}_{2} \mathrm{O}(7.0 \mathrm{mg}, 0.025 \mathrm{mmol}, 0.05$ equiv.), picolinic acid (6.2 mg, $0.05 \mathrm{mmol}$, 0.1 equiv.), lepidine (72.3 mg, $0.50 \mathrm{mmol}, 1.0$ equiv.), 2-methyl-3-phenylpropanoic acid (665.8 mg, 2.5 mmol, 5 equiv.), $\mathrm{NaBrO}_{3}(150.9 \mathrm{mg}, 1.0 \mathrm{mmol}, 2.0$ equiv.), $4.0 \mathrm{~mL}$ of $\mathrm{H}_{2} \mathrm{O}$ and $1.0 \mathrm{~mL}$ of DMSO, $440 \mathrm{~nm}$ LEDs were used. After 24 hours, the reaction mixture was subjected to the workup procedure outlined in the general procedure and purified by flash chromatography ( $5 \%$ ethyl acetate/hexanes) to provide the title compound as a light yellow oil (97.6 mg, 75\% yield). ${ }^{1} \mathrm{H}$ NMR (400 MHz, $\left.\mathrm{CDCl}_{3}\right) \delta 8.13$ $(\mathrm{d}, J=8.4 \mathrm{~Hz}, 1 \mathrm{H}), 7.96(\mathrm{~d}, J=8.3 \mathrm{~Hz}, 1 \mathrm{H}), 7.70(\mathrm{ddd}, J=8.4,6.8,1.5 \mathrm{~Hz}, 1 \mathrm{H}), 7.52$ (ddd, $J=8.2,6.8,1.3 \mathrm{~Hz}, 1 \mathrm{H}), 7.29-7.16(\mathrm{~m}, 5 \mathrm{H}), 7.13(\mathrm{~s}, 1 \mathrm{H}), 3.40(\mathrm{dq}, J=13.6,6.8$ Hz, 1H), $3.29(\mathrm{dd}, J=13.4,6.3 \mathrm{~Hz}, 1 \mathrm{H}), 2.94(\mathrm{dd}, J=13.4,8.6 \mathrm{~Hz}, 1 \mathrm{H}), 2.67$ (s, 3H), $1.38(\mathrm{~d}, J=6.9 \mathrm{~Hz}, 3 \mathrm{H}) ;{ }^{13} \mathrm{C} \mathrm{NMR}\left(101 \mathrm{MHz}, \mathrm{CDCl}_{3}\right) \delta 165.70,147.58,144.16,140.58$, $129.47,129.15,128.89,128.09,126.98,125.80,125.41,123.52,120.76,44.35,42.81$, 19.94, 18.70; HRMS (ESI) m/z calculated for $\mathrm{C}_{19} \mathrm{H}_{20} \mathrm{~N}\left[(\mathrm{M}+\mathrm{H})^{+}\right] 262.1590$, found 262.1589; IR (film) 3025, 2962, 1601, 1560, 1506, 1494, 1449, 1030, 862, $753 \mathrm{~cm}^{-1}$. 
Spectra data are consistent with those reported in the literature: Org. Lett. 2018, 20, 46864690.

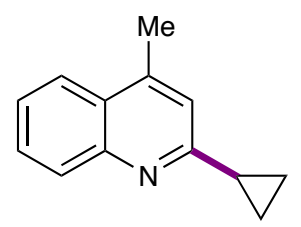

16

2-Cyclopropyl-4-methylquinoline (16): According to the general procedure B, $\mathrm{FeSO}_{4} \cdot 7 \mathrm{H}_{2} \mathrm{O}(14.0 \mathrm{mg}, 0.05 \mathrm{mmol}, 0.1$ equiv.), picolinic acid ( $12.4 \mathrm{mg}, 0.1 \mathrm{mmol}, 0.2$ equiv.), lepidine (72.3 mg, $0.50 \mathrm{mmol}, 1.0$ equiv.), cyclopropanecarboxylic acid ( $2.0 \mathrm{~mL}$, 25.0 mmol, 50 equiv.), $\mathrm{NaClO}_{3}$ ( $161.3 \mathrm{mg}, 1.5 \mathrm{mmol}, 3.0$ equiv.), $4.0 \mathrm{~mL}$ of $\mathrm{H}_{2} \mathrm{O}$ and 1.0 $\mathrm{mL}$ of DMSO, $390 \mathrm{~nm}$ LEDs were used. After 24 hours, the reaction mixture was subjected to the workup procedure outlined in the general procedure and purified by flash chromatography (5\% ethyl acetate/hexanes) to provide the title compound as a colorless oil (29.4 mg, 32\% yield). ${ }^{1} \mathrm{H}$ NMR (400 MHz, $\left.\mathrm{CDCl}_{3}\right) \delta 7.97$ (d, $\left.J=8.4 \mathrm{~Hz}, 1 \mathrm{H}\right), 7.90$ (d, $J=8.3 \mathrm{~Hz}, 1 \mathrm{H}), 7.63(\mathrm{t}, J=7.6 \mathrm{~Hz}, 1 \mathrm{H}), 7.45$ (t, $J=7.6 \mathrm{~Hz}, 1 \mathrm{H}), 6.99(\mathrm{~s}, 1 \mathrm{H}), 2.65(\mathrm{~s}$, $3 \mathrm{H}), 2.20(\mathrm{tt}, J=8.4,5.0 \mathrm{~Hz}, 1 \mathrm{H}), 1.17-1.10(\mathrm{~m}, 2 \mathrm{H}), 1.10-1.03(\mathrm{~m}, 2 \mathrm{H}) ;{ }^{13} \mathrm{C} \mathrm{NMR}$ $\left(101 \mathrm{MHz}, \mathrm{CDCl}_{3}\right) \delta 162.95,147.70,143.71,129.06,128.91,126.74,124.87,123.51$, 119.74, 18.64, 17.89, 9.91; HRMS (ESI) $\mathrm{m} / \mathrm{z}$ calculated for $\mathrm{C}_{13} \mathrm{H}_{14} \mathrm{~N}\left[(\mathrm{M}+\mathrm{H})^{+}\right]$184.1121, found 184.1120; IR (film) 3061, 3003, 1603, 1558, 1506, 1449, 1168, 1085, 1024, 955, $852,753 \mathrm{~cm}^{-1}$. Spectra data are consistent with those reported in the literature: Org. Lett. 2018, 20, 3229-3232. 


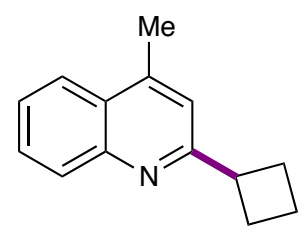

17

2-Cyclobutyl-4-methylquinoline (17): According to the general procedure A,

$\mathrm{FeSO}_{4} \cdot 7 \mathrm{H}_{2} \mathrm{O}(7.0 \mathrm{mg}, 0.025 \mathrm{mmol}, 0.05$ equiv.), picolinic acid ( $6.2 \mathrm{mg}, 0.05 \mathrm{mmol}, 0.1$

equiv.), lepidine (72.3 mg, $0.50 \mathrm{mmol}, 1.0$ equiv.), cyclobutanecarboxylic acid (510.8 mg, 5.0 mmol, 10 equiv.), $\mathrm{NaBrO}_{3}\left(226.4 \mathrm{mg}, 1.5 \mathrm{mmol}, 3.0\right.$ equiv.), $4.0 \mathrm{~mL}$ of $\mathrm{H}_{2} \mathrm{O}$ and 1.0 $\mathrm{mL}$ of DMSO, $440 \mathrm{~nm}$ LEDs were used. After 12 hours, the reaction mixture was subjected to the workup procedure outlined in the general procedure and purified by flash chromatography ( $5 \%$ ethyl acetate/hexanes) to provide the title compound as a colorless oil (73.8 mg, 75\% yield). ${ }^{1} \mathrm{H}$ NMR (400 MHz, $\left.\mathrm{CDCl}_{3}\right) \delta 8.07(\mathrm{~d}, J=8.3 \mathrm{~Hz}, 1 \mathrm{H}), 7.93(\mathrm{~d}$, $J=8.3 \mathrm{~Hz}, 1 \mathrm{H}), 7.66(\mathrm{ddd}, J=8.4,6.8,1.5 \mathrm{~Hz}, 1 \mathrm{H}), 7.49(\mathrm{ddd}, J=8.2,6.8,1.3 \mathrm{~Hz}, 1 \mathrm{H})$, $7.19(\mathrm{~s}, 1 \mathrm{H}), 3.83(\mathrm{p}, J=8.9 \mathrm{~Hz}, 1 \mathrm{H}), 2.68(\mathrm{~s}, 3 \mathrm{H}), 2.50-2.44(\mathrm{~m}, 2 \mathrm{H}), 2.44-2.40(\mathrm{~m}$, 2H), $2.19-2.05(\mathrm{~m}, 1 \mathrm{H}), 1.99-1.90(\mathrm{~m}, 1 \mathrm{H}) ;{ }^{13} \mathrm{C} \mathrm{NMR}\left(101 \mathrm{MHz}, \mathrm{CDCl}_{3}\right) \delta 164.62$, $147.42,144.20,129.37,128.95,126.81,125.37,123.49,120.15,42.54,28.18,18.72$, 18.30; HRMS (ESI) $\mathrm{m} / \mathrm{z}$ calculated for $\mathrm{C}_{14} \mathrm{H}_{16} \mathrm{~N}\left[(\mathrm{M}+\mathrm{H})^{+}\right]$198.1277, found 198.1276; IR (film) 3032, 2939, 2864, 1603, 1559, 1507, 1447, 1410, 1295, 1176, $757 \mathrm{~cm}^{-1}$. Spectra data are consistent with those reported in the literature: Org. Lett. 2018, 20, 3229-3232.<smiles>O=[N+]([O-])c1cc(C2CCCC2)nc2ccccc12</smiles>

18 
2-Cyclopentyl-4-methylquinoline (18): According to the general procedure B,

$\mathrm{FeSO}_{4} \cdot 7 \mathrm{H}_{2} \mathrm{O}$ (14.0 mg, $0.05 \mathrm{mmol}, 0.1$ equiv.), picolinic acid (12.4 mg, $0.1 \mathrm{mmol}, 0.2$ equiv.), lepidine (72.3 mg, $0.50 \mathrm{mmol}, 1.0$ equiv.), cyclopentanecarboxylic acid (582.3 mg, 5.0 mmol, 10 equiv.), $\mathrm{NaClO}_{3}$ (161.3 mg, $1.5 \mathrm{mmol}, 3.0$ equiv.), $3.0 \mathrm{~mL}$ of $\mathrm{H}_{2} \mathrm{O}$ and $2.0 \mathrm{~mL}$ of DMSO, $427 \mathrm{~nm}$ LEDs were used. After 24 hours, the reaction mixture was subjected to the workup procedure outlined in the general procedure and purified by flash chromatography ( $5 \%$ ethyl acetate/hexanes) to provide the title compound as a colorless oil (84.8 mg, 80\% yield). ${ }^{1} \mathrm{H}$ NMR (400 MHz, $\left.\mathrm{CDCl}_{3}\right) \delta 8.05(\mathrm{~d}, J=8.4 \mathrm{~Hz}, 1 \mathrm{H}), 7.93$ (d, $J=8.3 \mathrm{~Hz}, 1 \mathrm{H}), 7.66(\mathrm{ddd}, J=8.4,6.8,1.5 \mathrm{~Hz}, 1 \mathrm{H}), 7.48(\mathrm{ddd}, J=8.2,6.8,1.3 \mathrm{~Hz}, 1 \mathrm{H})$, $7.17(\mathrm{~s}, 1 \mathrm{H}), 3.34(\mathrm{p}, J=8.5,7.9 \mathrm{~Hz}, 1 \mathrm{H}), 2.67(\mathrm{~s}, 3 \mathrm{H}), 2.22-2.12(\mathrm{~m}, 2 \mathrm{H}), 1.93-1.82$ (m, 4H), $1.80-1.69(\mathrm{~m}, 2 \mathrm{H}) ;{ }^{13} \mathrm{C}$ NMR $\left(101 \mathrm{MHz}, \mathrm{CDCl}_{3}\right) \delta$ 165.84, 147.34, 144.16, 129.30, 128.89, 126.89, 125.31, 123.47, 120.55, 48.66, 33.52, 25.99, 18.76; HRMS (ESI) $\mathrm{m} / \mathrm{z}$ calculated for $\mathrm{C}_{15} \mathrm{H}_{18} \mathrm{~N}\left[(\mathrm{M}+\mathrm{H})^{+}\right]$212.1434, found 212.1433; IR (film) 2948, 2866, $1602,1559,1507,1410,1176,1032,861,754 \mathrm{~cm}^{-1}$. Spectra data are consistent with those reported in the literature: Org. Lett. 2018, 20, 3229-3232.

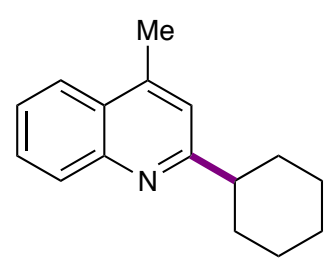

19

2-Cyclohexyl-4-methylquinoline (19): According to the general procedure A, $\mathrm{FeSO}_{4} \cdot 7 \mathrm{H}_{2} \mathrm{O}(7.0 \mathrm{mg}, 0.025 \mathrm{mmol}, 0.05$ equiv.), picolinic acid (6.2 mg, $0.05 \mathrm{mmol}, 0.1$ equiv.), lepidine (72.3 mg, $0.50 \mathrm{mmol}, 1.0$ equiv.), cyclohexanecarboxylic acid (647.3 mg, 5.0 mmol, 10 equiv.), $\mathrm{NaBrO}_{3}$ (150.9 mg, $1.0 \mathrm{mmol}, 2.0$ equiv.), $4.0 \mathrm{~mL}$ of $\mathrm{H}_{2} \mathrm{O}$ and $1.0 \mathrm{~mL}$ of DMSO, $440 \mathrm{~nm}$ LEDs were used. After 24 hours, the reaction mixture was 
subjected to the workup procedure outlined in the general procedure and purified by flash chromatography (5\% ethyl acetate/hexanes) to provide the title compound as a colorless oil (81.5 mg, 72\% yield). ${ }^{1} \mathrm{H}$ NMR (400 MHz, $\left.\mathrm{CDCl}_{3}\right) \delta 8.06(\mathrm{~d}, J=8.4 \mathrm{~Hz}, 1 \mathrm{H}), 7.93(\mathrm{~d}$, $J=8.3 \mathrm{~Hz}, 1 \mathrm{H}), 7.66(\mathrm{ddd}, J=8.4,6.9,1.5 \mathrm{~Hz}, 1 \mathrm{H}), 7.48(\mathrm{ddd}, J=8.2,6.8,1.3 \mathrm{~Hz}, 1 \mathrm{H})$, $7.16(\mathrm{~s}, 1 \mathrm{H}), 2.88(\mathrm{tt}, J=12.0,3.4 \mathrm{~Hz}, 1 \mathrm{H}), 2.67(\mathrm{~s}, 3 \mathrm{H}), 2.05-1.96(\mathrm{~m}, 2 \mathrm{H}), 1.93-1.84$ (m, 2H), $1.82-1.74(\mathrm{~m}, 1 \mathrm{H}), 1.62(\mathrm{qd}, J=12.4,2.8 \mathrm{~Hz}, 2 \mathrm{H}), 1.53-1.40(\mathrm{~m}, 2 \mathrm{H}), 1.39-$ $21.28(\mathrm{~m}, 1 \mathrm{H}) ;{ }^{13} \mathrm{C} \mathrm{NMR}\left(101 \mathrm{MHz}, \mathrm{CDCl}_{3}\right) \delta 166.44,147.49,144.27,129.38,128.91$, $126.98,125.33,123.51,120.19,47.52,32.78,26.51,26.07,18.80 ;$ HRMS (ESI) m/z calculated for $\mathrm{C}_{16} \mathrm{H}_{20} \mathrm{~N}\left[(\mathrm{M}+\mathrm{H})^{+}\right]$226.1590, found 226.1589; IR (film) 2925, 2851, 1604, $1559,1507,1448,1411,1342,954,860,758 \mathrm{~cm}^{-1}$. Spectra data are consistent with those reported in the literature: J. Am. Chem. Soc. 2017, 49, 188-194.

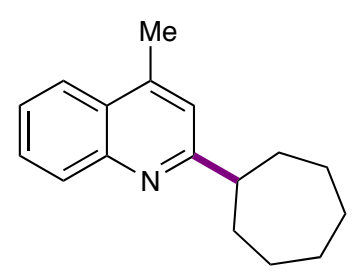

20

2-Cycloheptyl-4-methylquinoline (20): According to the general procedure A, $\mathrm{FeSO}_{4} \cdot 7 \mathrm{H}_{2} \mathrm{O}(7.0 \mathrm{mg}, 0.025 \mathrm{mmol}, 0.05$ equiv.), picolinic acid $(6.2 \mathrm{mg}, 0.05 \mathrm{mmol}, 0.1$ equiv.), lepidine (72.3 mg, $0.50 \mathrm{mmol}, 1.0$ equiv.), cycloheptanecarboxylic acid (710.0 mg, 5.0 mmol, 10 equiv.), $\mathrm{NaBrO}_{3}$ (226.4 mg, $1.5 \mathrm{mmol}, 3.0$ equiv.), $4.5 \mathrm{~mL}$ of $\mathrm{H}_{2} \mathrm{O}$ and $0.5 \mathrm{~mL}$ of DMSO, $456 \mathrm{~nm}$ LEDs were used. After 24 hours, the reaction mixture was subjected to the workup procedure outlined in the general procedure and purified by flash chromatography ( $10 \%$ ethyl acetate/hexanes) to provide the title compound as a colorless oil (96.2 mg, 81\% yield). ${ }^{1} \mathrm{H}$ NMR (400 MHz, $\left.\mathrm{CDCl}_{3}\right) \delta 8.05(\mathrm{~d}, J=8.4 \mathrm{~Hz}, 1 \mathrm{H}), 7.93$ (dd, $J=8.3,1.5 \mathrm{~Hz}, 1 \mathrm{H}), 7.66$ (ddd, $J=8.4,6.8,1.5 \mathrm{~Hz}, 1 \mathrm{H}$ ), 7.49 (ddd, $J=8.2,6.8,1.3$ 
Hz, 1H), 7.14 (s, 1H), 3.04 (tt, $J=10.1,3.3 \mathrm{~Hz}, 1 \mathrm{H}), 2.68(\mathrm{~s}, 3 \mathrm{H}), 2.08-1.99(\mathrm{~m}, 2 \mathrm{H})$, $1.91-1.78(\mathrm{~m}, 4 \mathrm{H}), 1.78-1.70(\mathrm{~m}, 2 \mathrm{H}), 1.69-1.59(\mathrm{~m}, 4 \mathrm{H}) ;{ }^{13} \mathrm{C} \mathrm{NMR}(101 \mathrm{MHz}$, $\left.\mathrm{CDCl}_{3}\right) \delta 168.05,147.21,144.52,129.30,128.98,126.91,125.35,123.51,120.26,49.49$, 35.04, 27.94, 27.44, 18.84; HRMS (ESI) $\mathrm{m} / \mathrm{z}$ calculated for $\mathrm{C}_{17} \mathrm{H}_{22} \mathrm{~N}\left[(\mathrm{M}+\mathrm{H})^{+}\right]$240.1747, found 240.1746; IR (film) 2919, 2852, 1602, 1560, 1506, 1445, 1411, 1340, 1026, 855, $754 \mathrm{~cm}^{-1}$.

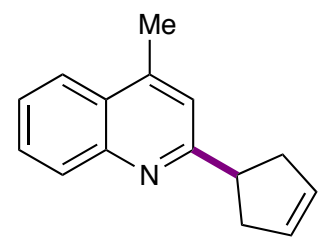

21

2-(Cyclopent-3-en-1-yl)-4-methylquinoline (21): According to the general procedure B, $\mathrm{FeSO}_{4} \cdot 7 \mathrm{H}_{2} \mathrm{O}$ (14.0 mg, $0.05 \mathrm{mmol}, 0.1$ equiv.), picolinic acid (12.4 mg, $0.1 \mathrm{mmol}, 0.2$ equiv.), lepidine (72.3 mg, $0.50 \mathrm{mmol}, 1.0$ equiv.), cyclopent-3-enecarboxylic acid (578.0 mg, 5.0 mmol, 10 equiv.), $\mathrm{NaClO}_{3}$ (161.3 mg, $1.5 \mathrm{mmol}, 3.0$ equiv.), $4.0 \mathrm{~mL}$ of $\mathrm{H}_{2} \mathrm{O}$ and $1.0 \mathrm{~mL}$ of DMSO, $440 \mathrm{~nm}$ LEDs were used. After 24 hours, the reaction mixture was subjected to the workup procedure outlined in the general procedure and purified by flash chromatography ( $5 \%$ ethyl acetate/hexanes) to provide the title compound as a yellow oil (61.7 mg, 59\% yield). ${ }^{1} \mathrm{H}$ NMR (400 MHz, $\left.\mathrm{CDCl}_{3}\right) \delta 8.05(\mathrm{~d}, J=8.4 \mathrm{~Hz}, 1 \mathrm{H}), 7.93(\mathrm{~d}, J$ $=8.3 \mathrm{~Hz}, 1 \mathrm{H}), 7.66(\mathrm{ddd}, J=8.4,6.9,1.5 \mathrm{~Hz}, 1 \mathrm{H}), 7.49(\mathrm{ddd}, J=8.2,6.8,1.3 \mathrm{~Hz}, 1 \mathrm{H})$, $7.19(\mathrm{~s}, 1 \mathrm{H}), 5.84-5.80(\mathrm{~m}, 2 \mathrm{H}), 3.82(\mathrm{tt}, J=9.5,6.5 \mathrm{~Hz}, 1 \mathrm{H}), 3.01-2.90(\mathrm{~m}, 2 \mathrm{H}), 2.75$ - $2.68(\mathrm{~m}, 2 \mathrm{H}), 2.67(\mathrm{~s}, 3 \mathrm{H}) ;{ }^{13} \mathrm{C} \mathrm{NMR}\left(101 \mathrm{MHz}, \mathrm{CDCl}_{3}\right) \delta 166.48,147.17,144.75$, $129.75,129.39,129.04,126.97,125.48,123.52,120.19,45.51,40.18,18.82$; HRMS (ESI) $\mathrm{m} / \mathrm{z}$ calculated for $\mathrm{C}_{15} \mathrm{H}_{16} \mathrm{~N}\left[(\mathrm{M}+\mathrm{H})^{+}\right]$210.1277, found 210.1276; IR (film) 2920, 2847, 
$1602,1559,1506,1444,1169,941,861,755 \mathrm{~cm}^{-1}$. Spectra data are consistent with those reported in the literature: Org. Lett. 2018, 20, 3229-3232.

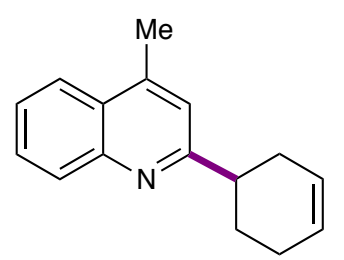

22

2-(Cyclohex-3-en-1-yl)-4-methylquinoline (22): According to the general procedure A, $\mathrm{FeSO}_{4} \cdot 7 \mathrm{H}_{2} \mathrm{O}(7.0 \mathrm{mg}, 0.025 \mathrm{mmol}, 0.05$ equiv.), picolinic acid (6.2 mg, $0.05 \mathrm{mmol}, 0.1$ equiv.), lepidine (72.3 mg, $0.50 \mathrm{mmol}, 1.0$ equiv.), cyclohex-3-enecarboxylic acid (643.6 mg, 5.0 mmol, 10 equiv.), $\mathrm{NaBrO}_{3}$ (150.9 mg, 1.0 mmol, 2.0 equiv.), $4.5 \mathrm{~mL}$ of $\mathrm{H}_{2} \mathrm{O}$ and $0.5 \mathrm{~mL}$ of DMSO, $456 \mathrm{~nm}$ LEDs were used. After 24 hours, the reaction mixture was subjected to the workup procedure outlined in the general procedure and purified by flash chromatography ( $5 \%$ ethyl acetate/hexanes) to provide the title compound as a colorless oil (64.9 mg, 58\% yield). ${ }^{1} \mathrm{H}$ NMR (400 MHz, $\left.\mathrm{CDCl}_{3}\right) \delta 8.06(\mathrm{~d}, J=8.4 \mathrm{~Hz}, 1 \mathrm{H}), 7.93$ (d, $J=8.3 \mathrm{~Hz}, 1 \mathrm{H}), 7.67(\mathrm{ddd}, J=8.4,6.9,1.5 \mathrm{~Hz}, 1 \mathrm{H}), 7.49(\mathrm{ddd}, J=8.3,6.8,1.3 \mathrm{~Hz}, 1 \mathrm{H})$, $7.17(\mathrm{~s}, 1 \mathrm{H}), 5.84-5.77(\mathrm{~m}, 2 \mathrm{H}), 3.15(\mathrm{dddd}, J=12.3,9.5,6.3,3.0 \mathrm{~Hz}, 1 \mathrm{H}), 2.67(\mathrm{~s}, 3 \mathrm{H})$, $2.48-2.35(\mathrm{~m}, 2 \mathrm{H}), 2.30-2.15(\mathrm{~m}, 2 \mathrm{H}), 2.12-2.03(\mathrm{~m}, 1 \mathrm{H}), 1.98-1.87(\mathrm{~m}, 1 \mathrm{H}) ;{ }^{13} \mathrm{C}$ NMR $\left(101 \mathrm{MHz}, \mathrm{CDCl}_{3}\right) \delta 165.80,147.47,144.36,129.35,128.93,126.94,126.81$, 126.32, 125.40, 123.48, 120.30, 42.99, 31.28, 28.50, 25.58, 18.76; HRMS (ESI) m/z calculated for $\mathrm{C}_{16} \mathrm{H}_{18} \mathrm{~N}\left[(\mathrm{M}+\mathrm{H})^{+}\right]$224.1434, found 224.1433; IR (film) 2920, 2835, 1603, $1560,1507,1448,1412,1186,1026,758 \mathrm{~cm}^{-1}$. Spectra data are consistent with those reported in the literature: CN109134362, 2019, A. 


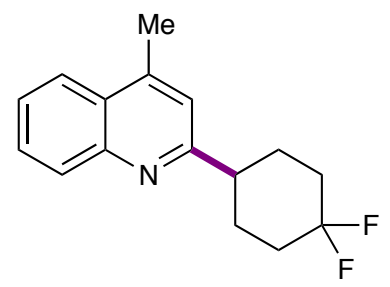

23

2-(4,4-Difluorocyclohexyl)-4-methylquinoline (23): According to the general procedure $\mathrm{B}, \mathrm{FeSO}_{4} \cdot 7 \mathrm{H}_{2} \mathrm{O}$ (14.0 mg, $0.05 \mathrm{mmol}, 0.1$ equiv.), picolinic acid (12.4 mg, $0.1 \mathrm{mmol}, 0.2$ equiv.), lepidine (72.3 mg, $0.50 \mathrm{mmol}, 1.0$ equiv.), 4,4-difluorocyclohexanecarboxylic acid (821.4 mg, 5.0 mmol, 10 equiv.), $\mathrm{NaClO}_{3}(161.3 \mathrm{mg}, 1.5 \mathrm{mmol}, 3.0$ equiv.), $4.0 \mathrm{~mL}$ of $\mathrm{H}_{2} \mathrm{O}$ and $1.0 \mathrm{~mL}$ of DMSO, $440 \mathrm{~nm}$ LEDs were used. After 24 hours, the reaction mixture was subjected to the workup procedure outlined in the general procedure and purified by flash chromatography (10\% ethyl acetate/hexanes) to provide the title compound as a white solid (74.5 mg, 57\% yield). ${ }^{1} \mathrm{H}$ NMR (400 MHz, $\left.\mathrm{CDCl}_{3}\right) \delta 8.04(\mathrm{~d}$, $J=8.4 \mathrm{~Hz}, 1 \mathrm{H}), 7.95(\mathrm{~d}, J=8.3 \mathrm{~Hz}, 1 \mathrm{H}), 7.68(\mathrm{t}, J=7.6 \mathrm{~Hz}, 1 \mathrm{H}), 7.52(\mathrm{t}, J=7.5 \mathrm{~Hz}, 1 \mathrm{H})$, $7.16(\mathrm{~s}, 1 \mathrm{H}), 2.97(\mathrm{tt}, J=12.1,3.7 \mathrm{~Hz}, 1 \mathrm{H}), 2.69(\mathrm{~s}, 3 \mathrm{H}), 2.34-2.19(\mathrm{~m}, 2 \mathrm{H}), 2.13-1.82$ $(\mathrm{m}, 6 \mathrm{H}) ;{ }^{13} \mathrm{C} \mathrm{NMR}\left(101 \mathrm{MHz}, \mathrm{CDCl}_{3}\right) \delta 163.80$ (d), 147.49, 144.72, 129.43, 129.16, 127.07, 125.71, 123.58, 123.20 (dd), 119.73, 45.07 (d), 33.67 (dd), 28.67 (d), 18.81; HRMS (ESI) $\mathrm{m} / \mathrm{z}$ calculated for $\mathrm{C}_{16} \mathrm{H}_{18} \mathrm{~F}_{2} \mathrm{~N}\left[(\mathrm{M}+\mathrm{H})^{+}\right]$262.1402, found 262.1405; IR (film) $2957,2870,1603,1446,1367,1269,1099,955,940,756 \mathrm{~cm}^{-1}$. Spectra data are consistent with those reported in the literature: Org. Lett. 2018, 20, 4686-4690.<smiles>Cc1cc(C2CCC(=O)CC2)nc2ccccc12</smiles> 
4-(4-Methylquinolin-2-yl)cyclohexan-1-one (24): According to the general procedure B, $\mathrm{FeSO}_{4} \cdot 7 \mathrm{H}_{2} \mathrm{O}$ (14.0 mg, $0.05 \mathrm{mmol}, 0.1$ equiv.), picolinic acid (12.4 mg, $0.1 \mathrm{mmol}, 0.2$ equiv.), lepidine (72.3 mg, $0.50 \mathrm{mmol}, 1.0$ equiv.), 4-oxocyclohexanecarboxylic acid (732.7 mg, 5.0 mmol, 10 equiv.), $\mathrm{NaClO}_{3}$ (161.3 mg, $1.5 \mathrm{mmol}, 3.0$ equiv.), $4.0 \mathrm{~mL}$ of $\mathrm{H}_{2} \mathrm{O}$ and $1.0 \mathrm{~mL}$ of DMSO, $440 \mathrm{~nm}$ LEDs were used. After 24 hours, the reaction mixture was subjected to the workup procedure outlined in the general procedure and purified by flash chromatography ( $33 \%$ ethyl acetate/hexanes) to provide the title compound as a white solid (95.5 mg, 80\% yield). ${ }^{1} \mathrm{H}$ NMR (400 MHz, $\left.\mathrm{CDCl}_{3}\right) \delta 8.02(\mathrm{~d}$, $J=8.4 \mathrm{~Hz}, 1 \mathrm{H}), 7.95(\mathrm{~d}, J=8.3 \mathrm{~Hz}, 1 \mathrm{H}), 7.67(\mathrm{t}, J=7.6 \mathrm{~Hz}, 1 \mathrm{H}), 7.51(\mathrm{t}, J=7.6 \mathrm{~Hz}, 1 \mathrm{H})$, $7.16(\mathrm{~s}, 1 \mathrm{H}), 3.33(\mathrm{tt}, J=11.5,3.6 \mathrm{~Hz}, 1 \mathrm{H}), 2.68(\mathrm{~s}, 3 \mathrm{H}), 2.60-2.47(\mathrm{~m}, 4 \mathrm{H}), 2.39-2.30$ $(\mathrm{m}, 2 \mathrm{H}), 2.23-2.11(\mathrm{~m}, 2 \mathrm{H}) ;{ }^{13} \mathrm{C} \mathrm{NMR}\left(101 \mathrm{MHz}, \mathrm{CDCl}_{3}\right) \delta 211.10,163.39,147.48$, $144.74,129.41,129.18,127.03,125.73,123.56,119.93,44.90,40.89,32.16,18.79$; HRMS (ESI) m/z calculated for $\mathrm{C}_{16} \mathrm{H}_{18} \mathrm{NO}\left[(\mathrm{M}+\mathrm{H})^{+}\right]$240.1383, found 240.1382; IR (film) $2930,2870,1709,1597,1506,1447,1280,1163,947,872,763 \mathrm{~cm}^{-1}$.

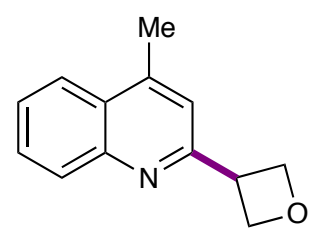

25

4-Methyl-2-(oxetan-3-yl)quinoline (25): According to the general procedure A, $\mathrm{FeSO}_{4} \cdot 7 \mathrm{H}_{2} \mathrm{O}(7.0 \mathrm{mg}, 0.025 \mathrm{mmol}, 0.05$ equiv.), picolinic acid (6.2 mg, $0.05 \mathrm{mmol}, 0.1$ equiv.), lepidine (72.3 mg, $0.50 \mathrm{mmol}, 1.0$ equiv.), oxetane-3-carboxylic acid (537.3 mg, 5.0 mmol, 10 equiv.), $\mathrm{NaBrO}_{3}$ (150.9 mg, $1.0 \mathrm{mmol}, 2.0$ equiv.), $4.0 \mathrm{~mL}$ of $\mathrm{H}_{2} \mathrm{O}$ and 1.0 $\mathrm{mL}$ of DMSO, $440 \mathrm{~nm}$ LEDs were used. After 24 hours, the reaction mixture was subjected to the workup procedure outlined in the general procedure and purified by flash 
chromatography ( $20 \%$ ethyl acetate/hexanes) to provide the title compound as a white solid (32.5 mg, 33\% yield). ${ }^{1} \mathrm{H}$ NMR (400 MHz, $\left.\mathrm{CDCl}_{3}\right) \delta 8.07(\mathrm{~d}, J=8.4 \mathrm{~Hz}, 1 \mathrm{H}), 7.97$ (d, $J=8.3 \mathrm{~Hz}, 1 \mathrm{H}), 7.70$ (ddd, $J=8.4,6.8,1.4 \mathrm{~Hz}, 1 \mathrm{H}), 7.54$ (ddd, $J=8.3,6.9,1.3 \mathrm{~Hz}$, 1H), $7.37(\mathrm{~s}, 1 \mathrm{H}), 5.15(\mathrm{dd}, J=8.4,6.0 \mathrm{~Hz}, 2 \mathrm{H}), 5.04(\mathrm{t}, J=6.3 \mathrm{~Hz}, 2 \mathrm{H}), 4.51(\mathrm{tt}, J=8.5$, $6.5 \mathrm{~Hz}, 1 \mathrm{H}), 2.72(\mathrm{~s}, 3 \mathrm{H}) ;{ }^{13} \mathrm{C} \mathrm{NMR}\left(101 \mathrm{MHz}, \mathrm{CDCl}_{3}\right) \delta 160.43,147.45,145.30,129.55$, 129.37, 127.11, 126.00, 123.59, 119.86, 76.75, 42.42, 18.78; HRMS (ESI) m/z calculated for $\mathrm{C}_{13} \mathrm{H}_{14} \mathrm{NO}\left[(\mathrm{M}+\mathrm{H})^{+}\right]$200.1070, found 200.1071; IR (film) 2935, 2867, 1602, 1506, $1447,1291,1108,981,846,756 \mathrm{~cm}^{-1}$. Spectra data are consistent with those reported in the literature: J. Org. Chem. 2009, 74, 6354-6357.

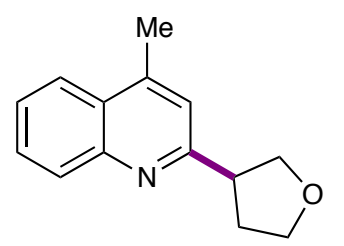

26

4-Methyl-2-(tetrahydrofuran-3-yl)quinoline (26): According to the general procedure A, $\mathrm{FeSO}_{4} \cdot 7 \mathrm{H}_{2} \mathrm{O}(7.0 \mathrm{mg}, 0.025 \mathrm{mmol}, 0.05$ equiv.), picolinic acid $(6.2 \mathrm{mg}, 0.05 \mathrm{mmol}$, 0.1 equiv.), lepidine (72.3 mg, $0.50 \mathrm{mmol}, 1.0$ equiv.), tetrahydrofuran-3-carboxylic acid (598.6 mg, $5.0 \mathrm{mmol}, 10$ equiv.), $\mathrm{NaBrO}_{3}(150.9 \mathrm{mg}, 1.0 \mathrm{mmol}, 2.0$ equiv.), $4.0 \mathrm{~mL}$ of $\mathrm{H}_{2} \mathrm{O}$ and $1.0 \mathrm{~mL}$ of DMSO, $440 \mathrm{~nm}$ LEDs were used. After 24 hours, the reaction mixture was subjected to the workup procedure outlined in the general procedure and purified by flash chromatography ( $20 \%$ ethyl acetate/hexanes) to provide the title compound as a colorless oil (71.4 mg, 67\% yield). ${ }^{1} \mathrm{H}$ NMR (400 MHz, $\left.\mathrm{CDCl}_{3}\right) \delta 8.03(\mathrm{~d}$, $J=8.2 \mathrm{~Hz}, 1 \mathrm{H}), 7.94(\mathrm{dd}, J=8.4,1.4 \mathrm{~Hz}, 1 \mathrm{H}), 7.67$ (ddd, $J=8.4,6.9,1.5 \mathrm{~Hz}, 1 \mathrm{H}), 7.51$

(ddd, $J=8.2,6.8,1.3 \mathrm{~Hz}, 1 \mathrm{H}), 7.20(\mathrm{~s}, 1 \mathrm{H}), 4.22(\mathrm{t}, J=8.1 \mathrm{~Hz}, 1 \mathrm{H}), 4.15(\mathrm{td}, J=8.3,4.8$ Hz, 1H), 4.05 (dd, $J=8.6,6.6 \mathrm{~Hz}, 1 \mathrm{H}), 3.94(\mathrm{q}, J=7.6 \mathrm{~Hz}, 1 \mathrm{H}), 3.74$ (p, $J=7.5 \mathrm{~Hz}, 1 \mathrm{H})$, 
$2.68(\mathrm{~s}, 3 \mathrm{H}), 2.50-2.41(\mathrm{~m}, 1 \mathrm{H}), 2.29(\mathrm{dq}, J=12.4,7.7 \mathrm{~Hz}, 1 \mathrm{H}) ;{ }^{13} \mathrm{C} \mathrm{NMR}(101 \mathrm{MHz}$, $\left.\mathrm{CDCl}_{3}\right) \delta 162.47,147.36,144.84,129.45,129.16,127.00,125.72,123.53,120.53,73.48$, 68.71, 47.61, 33.40, 18.75; HRMS (ESI) m/z calculated for $\mathrm{C}_{14} \mathrm{H}_{16} \mathrm{NO}\left[(\mathrm{M}+\mathrm{H})^{+}\right]$ 214.1226, found 214.1226; IR (film) 2946, 2895, 1601, 1560, 1507, 1446, 1175, 1061, $905,862,756 \mathrm{~cm}^{-1}$. Spectra data are consistent with those reported in the literature: Org. Lett. 2018, 20, 3229-3232.

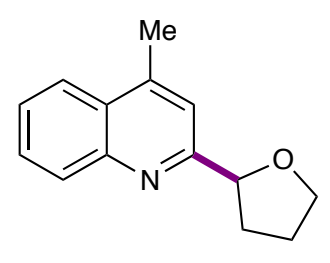

27

4-Methyl-2-(tetrahydrofuran-2-yl)quinoline (27): According to the general procedure A, $\mathrm{FeSO}_{4} \cdot 7 \mathrm{H}_{2} \mathrm{O}$ (7.0 mg, $0.025 \mathrm{mmol}, 0.05$ equiv.), picolinic acid ( $6.2 \mathrm{mg}, 0.05 \mathrm{mmol}$, 0.1 equiv.), lepidine (72.3 mg, $0.50 \mathrm{mmol}, 1.0$ equiv.), tetrahydrofuran-2-carboxylic acid (586.5 mg, $5.0 \mathrm{mmol}, 10$ equiv.), $\mathrm{NaBrO}_{3}(150.9 \mathrm{mg}, 1.0 \mathrm{mmol}, 2.0$ equiv.), $4.5 \mathrm{~mL}$ of $\mathrm{H}_{2} \mathrm{O}$ and $0.5 \mathrm{~mL}$ of DMSO, $456 \mathrm{~nm}$ LEDs were used. After 24 hours, the reaction mixture was subjected to the workup procedure outlined in the general procedure and purified by flash chromatography ( $33 \%$ ethyl acetate/hexanes) to provide the title compound as a white solid ( $85.7 \mathrm{mg}, 80 \%$ yield). ${ }^{1} \mathrm{H} \mathrm{NMR}\left(400 \mathrm{MHz}, \mathrm{CDCl}_{3}\right) \delta 8.04(\mathrm{~d}$, $J=8.4 \mathrm{~Hz}, 1 \mathrm{H}), 7.93(\mathrm{~d}, J=7.8 \mathrm{~Hz}, 1 \mathrm{H}), 7.65$ (ddd $J=8.4,6.8,1.4 \mathrm{~Hz}, 1 \mathrm{H}), 7.48$ (ddd, $J=8.2,6.9,1.3 \mathrm{~Hz}, 1 \mathrm{H}), 7.42(\mathrm{~s}, 1 \mathrm{H}), 5.12(\mathrm{t}, J=7.0 \mathrm{~Hz}, 1 \mathrm{H}), 4.15(\mathrm{dt}, J=8.1,6.5 \mathrm{~Hz}$, 1H), $4.01(\mathrm{dt}, J=8.2,6.7 \mathrm{~Hz}, 1 \mathrm{H}), 2.67(\mathrm{~s}, 3 \mathrm{H}), 2.54-2.43(\mathrm{~m}, 1 \mathrm{H}), 2.09-1.94(\mathrm{~m}, 3 \mathrm{H})$; ${ }^{13} \mathrm{C}$ NMR (101 MHz, $\left.\mathrm{CDCl}_{3}\right) \delta 162.94,147.16,144.80,129.38,128.99,127.30,125.68$, 123.55, 118.45, 81.93, 69.11, 33.18, 25.84, 18.75; HRMS (ESI) m/z calculated for $\mathrm{C}_{14} \mathrm{H}_{16} \mathrm{NO}\left[(\mathrm{M}+\mathrm{H})^{+}\right]$214.1226, found 214.1226; IR (film) 2972, 2868, 1600, 1561, 1508, 
$1446,1063,880,863,755 \mathrm{~cm}^{-1}$. Spectra data are consistent with those reported in the literature: Synlett 2016, 27, 1282-1286.

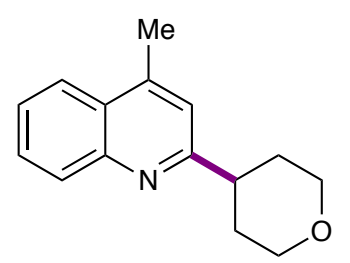

28

4-Methyl-2-(tetrahydro-2H-pyran-4-yl)quinoline (28): According to the general procedure $\mathrm{A}, \mathrm{FeSO}_{4} \cdot 7 \mathrm{H}_{2} \mathrm{O}(7.0 \mathrm{mg}, 0.025 \mathrm{mmol}, 0.05$ equiv.), picolinic acid (6.2 $\mathrm{mg}$, $0.05 \mathrm{mmol}, 0.1$ equiv.), lepidine (72.3 mg, $0.50 \mathrm{mmol}, 1.0$ equiv.), tetrahydro- $2 H$-pyran4-carboxylic acid (664.0 mg, 5.0 mmol, 10 equiv.), $\mathrm{NaBrO}_{3}$ (150.9 mg, 1.0 mmol, 2.0 equiv.), $4.5 \mathrm{~mL}$ of $\mathrm{H}_{2} \mathrm{O}$ and $0.5 \mathrm{~mL}$ of DMSO, $456 \mathrm{~nm}$ LEDs were used. After 24 hours, the reaction mixture was subjected to the workup procedure outlined in the general procedure and purified by flash chromatography (20\% ethyl acetate/hexanes) to provide the title compound as a white solid $\left(68.3 \mathrm{mg}, 60 \%\right.$ yield). ${ }^{1} \mathrm{H} \mathrm{NMR}\left(400 \mathrm{MHz}, \mathrm{CDCl}_{3}\right) \delta$ $8.04(\mathrm{~d}, J=8.4 \mathrm{~Hz}, 1 \mathrm{H}), 7.94(\mathrm{~d}, J=8.3 \mathrm{~Hz}, 1 \mathrm{H}), 7.67(\mathrm{t}, J=7.6 \mathrm{~Hz}, 1 \mathrm{H}), 7.50(\mathrm{t}, J=7.5$ $\mathrm{Hz}, 1 \mathrm{H}), 7.17(\mathrm{~s}, 1 \mathrm{H}), 4.12(\mathrm{dd}, J=10.6,3.8 \mathrm{~Hz}, 2 \mathrm{H}), 3.59(\mathrm{td}, J=11.7,2.3 \mathrm{~Hz}, 2 \mathrm{H})$, $3.12(\mathrm{tt}, J=12.0,4.0 \mathrm{~Hz}, 1 \mathrm{H}), 2.69(\mathrm{~s}, 3 \mathrm{H}), 2.01(\mathrm{qd}, J=12.2,4.3 \mathrm{~Hz}, 2 \mathrm{H}), 1.95-1.84$ $(\mathrm{m}, 2 \mathrm{H}) ;{ }^{13} \mathrm{C} \mathrm{NMR}\left(101 \mathrm{MHz}, \mathrm{CDCl}_{3}\right) \delta 164.17,147.53,144.64,129.44,129.10,127.04$, 125.62, 123.56, 119.83, 68.08, 44.35, 32.24, 18.84; HRMS (ESI) m/z calculated for $\mathrm{C}_{15} \mathrm{H}_{18} \mathrm{NO}\left[(\mathrm{M}+\mathrm{H})^{+}\right] 228.1383$, found 228.1382; IR (film) 2957, 1936, 1600, 1442, 1273, $1125,1115,1019,879,770 \mathrm{~cm}^{-1}$. Spectra data are consistent with those reported in the literature: Org. Lett. 2018, 20, 3229-3232. 


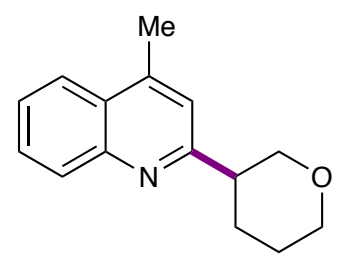

29

4-Methyl-2-(tetrahydro-2H-pyran-3-yl)quinoline (29): According to the general procedure $\mathrm{A}, \mathrm{FeSO}_{4} \cdot 7 \mathrm{H}_{2} \mathrm{O}(7.0 \mathrm{mg}, 0.025 \mathrm{mmol}, 0.05$ equiv.), picolinic acid $(6.2 \mathrm{mg}$, $0.05 \mathrm{mmol}, 0.1$ equiv.), lepidine ( $72.3 \mathrm{mg}, 0.50 \mathrm{mmol}, 1.0$ equiv.), tetrahydro- $2 H$-pyran3-carboxylic acid (670.8 mg, 5.0 mmol, 10 equiv.), $\mathrm{NaBrO}_{3}$ (150.9 mg, 1.0 mmol, 2.0 equiv.), $4.5 \mathrm{~mL}$ of $\mathrm{H}_{2} \mathrm{O}$ and $0.5 \mathrm{~mL}$ of DMSO, $456 \mathrm{~nm}$ LEDs were used. After 24 hours, the reaction mixture was subjected to the workup procedure outlined in the general procedure and purified by flash chromatography (20\% ethyl acetate/hexanes) to provide the title compound as a colorless oil $(84.6 \mathrm{mg}, 75 \%$ yield $) .{ }^{1} \mathrm{H}$ NMR $\left(400 \mathrm{MHz}, \mathrm{CDCl}_{3}\right) \delta$ $8.04(\mathrm{~d}, J=8.4 \mathrm{~Hz}, 1 \mathrm{H}), 7.91(\mathrm{~d}, J=8.3 \mathrm{~Hz}, 1 \mathrm{H}), 7.65(\mathrm{t}, J=7.6 \mathrm{~Hz}, 1 \mathrm{H}), 7.47(\mathrm{t}, J=7.5$ $\mathrm{Hz}, 1 \mathrm{H}), 7.13(\mathrm{~s}, 1 \mathrm{H}), 4.18(\mathrm{dd}, J=11.3,2.2 \mathrm{~Hz}, 1 \mathrm{H}), 4.05-3.95(\mathrm{~m}, 1 \mathrm{H}), 3.73(\mathrm{t}, J=$ $10.9 \mathrm{~Hz}, 1 \mathrm{H}), 3.52(\mathrm{td}, J=11.4,2.2 \mathrm{~Hz}, 1 \mathrm{H}), 3.16(\mathrm{tt}, J=11.0,4.1 \mathrm{~Hz}, 1 \mathrm{H}), 2.64(\mathrm{~s}, 3 \mathrm{H})$, $2.21-2.09(\mathrm{~m}, 1 \mathrm{H}), 2.00(\mathrm{qd}, J=12.2,4.2 \mathrm{~Hz}, 1 \mathrm{H}), 1.88-1.68(\mathrm{~m}, 2 \mathrm{H}) ;{ }^{13} \mathrm{C} \mathrm{NMR}(101$ $\left.\mathrm{MHz}, \mathrm{cdcl}_{3}\right) \delta 161.91,147.58,144.23,129.46,128.92,126.90,125.53,123.45,120.95$ 72.05, 68.04, 45.17, 29.24, 25.75, 18.60; HRMS (ESI) m/z calculated for $\mathrm{C}_{15} \mathrm{H}_{18} \mathrm{NO}$ $\left[(\mathrm{M}+\mathrm{H})^{+}\right]$228.1383, found 228.1382; IR (film) 2938, 2846, 1603, 1507, 1465, 1449, $1144,1102,1085,1026,759 \mathrm{~cm}^{-1}$.<smiles>Cc1cc(C2CCCCO2)nc2ccccc12</smiles> 
4-Methyl-2-(tetrahydro-2H-pyran-2-yl)quinoline (30): According to the general procedure $\mathrm{A}, \mathrm{FeSO}_{4} \cdot 7 \mathrm{H}_{2} \mathrm{O}(7.0 \mathrm{mg}, 0.025 \mathrm{mmol}, 0.05$ equiv.), picolinic acid $(6.2 \mathrm{mg}$, $0.05 \mathrm{mmol}, 0.1$ equiv.), lepidine (72.3 mg, $0.50 \mathrm{mmol}, 1.0$ equiv.), tetrahydro- $2 H$-pyran2-carboxylic acid (684.9 mg, 5.0 mmol, 10 equiv.), $\mathrm{NaBrO}_{3}$ (150.9 mg, $1.0 \mathrm{mmol}, 2.0$ equiv.), $4.0 \mathrm{~mL}$ of $\mathrm{H}_{2} \mathrm{O}$ and $1.0 \mathrm{~mL}$ of DMSO, $440 \mathrm{~nm}$ LEDs were used. After 24 hours, the reaction mixture was subjected to the workup procedure outlined in the general procedure and purified by flash chromatography (20\% ethyl acetate/hexanes) to provide the title compound as a colorless oil $(80.5 \mathrm{mg}, 71 \%$ yield $) .{ }^{1} \mathrm{H} \mathrm{NMR}\left(400 \mathrm{MHz}, \mathrm{CDCl}_{3}\right) \delta$ $8.06(\mathrm{~d}, J=8.4 \mathrm{~Hz}, 1 \mathrm{H}), 7.96(\mathrm{~d}, J=8.3 \mathrm{~Hz}, 1 \mathrm{H}), 7.67(\mathrm{t}, J=7.6 \mathrm{~Hz}, 1 \mathrm{H}), 7.51(\mathrm{t}, J=7.6$ $\mathrm{Hz}, 1 \mathrm{H}), 7.46(\mathrm{~s}, 1 \mathrm{H}), 4.61(\mathrm{dd}, J=11.1,2.4 \mathrm{~Hz}, 1 \mathrm{H}), 4.21(\mathrm{dd}, J=11.4,4.1 \mathrm{~Hz}, 1 \mathrm{H})$, $3.69(\mathrm{td}, J=11.5,2.5 \mathrm{~Hz}, 1 \mathrm{H}), 2.70(\mathrm{~s}, 3 \mathrm{H}), 2.14-2.06(\mathrm{~m}, 1 \mathrm{H}), 2.02-1.92(\mathrm{~m}, 1 \mathrm{H})$, $1.82-1.69(\mathrm{~m}, 2 \mathrm{H}), 1.69-1.55(\mathrm{~m}, 2 \mathrm{H}) ;{ }^{13} \mathrm{C} \mathrm{NMR}\left(101 \mathrm{MHz}, \mathrm{CDCl}_{3}\right) \delta 162.07,147.04$, $145.02,129.52,129.04,127.46,125.80,123.60,118.75,81.56,68.86,32.75,25.80,23.67$, 18.81; HRMS (ESI) m/z calculated for $\mathrm{C}_{15} \mathrm{H}_{18} \mathrm{NO}\left[(\mathrm{M}+\mathrm{H})^{+}\right]$228.1383, found 228.1382; IR (film) 2935, 2848, 1603, 1507, 1440, 1227, 1202, 1089, 1046, 909, $757 \mathrm{~cm}^{-1}$. Spectra data are consistent with those reported in the literature: Angew. Chem. Int. Ed. 2015, 54, 1565-1569.

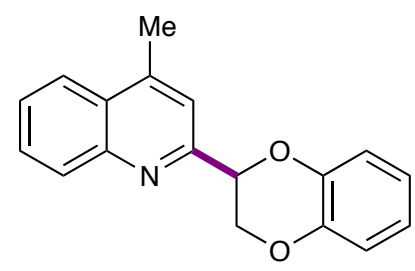

31

2-(2,3-Dihydrobenzo[b][1,4]dioxin-2-yl)-4-methylquinoline (31): According to the general procedure $\mathrm{A}, \mathrm{FeSO}_{4} \cdot 7 \mathrm{H}_{2} \mathrm{O}(7.0 \mathrm{mg}, 0.025 \mathrm{mmol}, 0.05$ equiv.), picolinic acid (6.2 mg, 0.05 mmol, 0.1 equiv.), lepidine (72.3 mg, 0.50 mmol, 1.0 equiv.), 2,3- 
dihydrobenzo[b][1,4]dioxine-2-carboxylic acid (908.6 mg, 5.0 mmol, 10 equiv.), $\mathrm{NaBrO}_{3}$ (150.9 mg, $1.0 \mathrm{mmol}, 2.0$ equiv.), $4.0 \mathrm{~mL}$ of $\mathrm{H}_{2} \mathrm{O}$ and $1.0 \mathrm{~mL}$ of DMSO, $440 \mathrm{~nm}$ LEDs were used. After 24 hours, the reaction mixture was subjected to the workup procedure outlined in the general procedure and purified by flash chromatography (5\% ethyl acetate/hexanes) to provide the title compound as a white solid (101.7 mg, $73 \%$ yield). ${ }^{1} \mathrm{H}$ NMR (400 MHz, $\left.\mathrm{CDCl}_{3}\right) \delta 8.10(\mathrm{~d}, J=8.4 \mathrm{~Hz}, 1 \mathrm{H}), 8.01(\mathrm{~d}, J=8.3 \mathrm{~Hz}, 1 \mathrm{H}), 7.73$ (ddd, $J=8.4,6.8,1.4 \mathrm{~Hz}, 1 \mathrm{H}), 7.58(\mathrm{ddd}, J=8.3,6.8,1.3 \mathrm{~Hz}, 1 \mathrm{H}), 7.54(\mathrm{~s}, 1 \mathrm{H}), 7.11-7.06(\mathrm{~m}$, 1H), $6.99-6.95(\mathrm{~m}, 1 \mathrm{H}), 6.95-6.87(\mathrm{~m}, 2 \mathrm{H}), 5.43(\mathrm{dd}, J=8.0,2.6 \mathrm{~Hz}, 1 \mathrm{H}), 4.72(\mathrm{dd}, J$ $=11.3,2.6 \mathrm{~Hz}, 1 \mathrm{H}), 4.31(\mathrm{dd}, J=11.3,8.0 \mathrm{~Hz}, 1 \mathrm{H}), 2.74(\mathrm{~s}, 3 \mathrm{H}) ;{ }^{13} \mathrm{C} \mathrm{NMR}(101 \mathrm{MHz}$, $\left.\mathrm{CDCl}_{3}\right) \delta 156.31,147.29,145.69,143.41,143.27,129.67,129.52,127.69,126.52,123.75$, 123.73, 121.61, 118.97, 117.44, 117.30, 75.97, 67.90, 18.96; HRMS (ESI) m/z calculated for $\mathrm{C}_{18} \mathrm{H}_{16} \mathrm{NO}_{2}\left[(\mathrm{M}+\mathrm{H})^{+}\right]$278.1176, found 278.1174; IR (film) 3062, 2922, 2870, 1594, $1493,1306,1291,1257,1130,1112,781 \mathrm{~cm}^{-1}$. Spectra data are consistent with those reported in the literature: CN109134362, 2019, A.

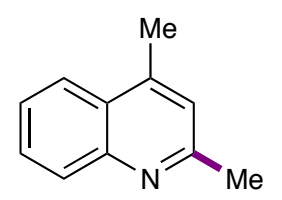

32

2,4-dimethylquinoline (32): According to the general procedure $\mathrm{B}, \mathrm{FeSO}_{4} \cdot 7 \mathrm{H}_{2} \mathrm{O}(14.0$ $\mathrm{mg}, 0.05 \mathrm{mmol}, 0.1$ equiv.), picolinic acid (12.4 mg, $0.1 \mathrm{mmol}, 0.2$ equiv.), lepidine (72.3 mg, 0.50 mmol, 1.0 equiv.), acetic acid ( $1.5 \mathrm{~mL}, 25.0 \mathrm{mmol}$, 50 equiv.), $\mathrm{NaClO}_{3}(161.3$ mg, $1.5 \mathrm{mmol}$, 3.0 equiv.), $4.0 \mathrm{~mL}$ of $\mathrm{H}_{2} \mathrm{O}$ and $1.0 \mathrm{~mL}$ of DMSO, $390 \mathrm{~nm}$ LEDs were used. After 24 hours, the reaction mixture was subjected to the workup procedure outlined in the general procedure and purified by flash chromatography (10\% ethyl 
acetate/hexanes) to provide the title compound as a colorless oil (30.7 mg, 39\% yield). ${ }^{1} \mathrm{H}$ NMR $\left(400 \mathrm{MHz}, \mathrm{CDCl}_{3}\right) \delta 8.01(\mathrm{~d}, J=8.4 \mathrm{~Hz}, 1 \mathrm{H}), 7.93(\mathrm{~d}, J=8.3 \mathrm{~Hz}, 1 \mathrm{H}), 7.66(\mathrm{t}, J=$ $7.6 \mathrm{~Hz}, 1 \mathrm{H}), 7.49(\mathrm{t}, J=7.5 \mathrm{~Hz}, 1 \mathrm{H}), 7.12(\mathrm{~s}, 1 \mathrm{H}), 2.69(\mathrm{~s}, 3 \mathrm{H}), 2.65(\mathrm{~s}, 3 \mathrm{H}) ;{ }^{13} \mathrm{C} \mathrm{NMR}$ $\left(101 \mathrm{MHz}, \mathrm{CDCl}_{3}\right) \delta 158.60,147.58,144.16,129.06,129.03,126.48,125.36,123.53$, 122.66, 25.17, 18.55; HRMS (ESI) $\mathrm{m} / \mathrm{z}$ calculated for $\mathrm{C}_{11} \mathrm{H}_{12} \mathrm{~N}\left[(\mathrm{M}+\mathrm{H})^{+}\right] 158.0964$, found 158.0963; IR (film) 2920, 2853, 1602, 1562, 1509, 1445, 1406, 1227, 1024, 858 $\mathrm{cm}^{-1}$. Spectra data are consistent with those reported in the literature: Synthesis 2017, 49, 188-194.

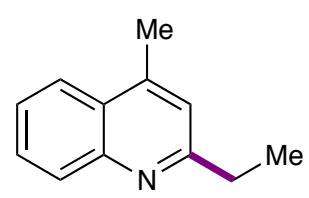

33

2-Ethyl-4-methylquinoline (33): According to the general procedure $\mathrm{A}, \mathrm{FeSO}_{4} \cdot 7 \mathrm{H}_{2} \mathrm{O}$ (7.0 mg, $0.025 \mathrm{mmol}, 0.05$ equiv.), picolinic acid (6.2 mg, $0.05 \mathrm{mmol}, 0.1$ equiv.), lepidine (72.3 mg, $0.50 \mathrm{mmol}, 1.0$ equiv.), propionic acid (374.1 mg, $5.0 \mathrm{mmol}, 10$ equiv.), $\mathrm{NaBrO}_{3}$ (150.9 mg, $1.0 \mathrm{mmol}, 2.0$ equiv.), $4.0 \mathrm{~mL}$ of $\mathrm{H}_{2} \mathrm{O}$ and $1.0 \mathrm{~mL}$ of DMSO, $440 \mathrm{~nm}$ LEDs were used. After 24 hours, the reaction mixture was subjected to the workup procedure outlined in the general procedure and purified by flash chromatography ( $10 \%$ ethyl acetate/hexanes) to provide the title compound as a colorless oil (66.5 mg, 78\% yield). ${ }^{1} \mathrm{H}$ NMR (400 MHz, $\left.\mathrm{CDCl}_{3}\right) \delta 8.08(\mathrm{~d}, J=8.4 \mathrm{~Hz}, 1 \mathrm{H}), 7.96$ (d, $J=8.3 \mathrm{~Hz}, 1 \mathrm{H}), 7.68(\mathrm{t}, J=7.6 \mathrm{~Hz}, 1 \mathrm{H}), 7.51(\mathrm{t}, J=7.6 \mathrm{~Hz}, 1 \mathrm{H}), 7.17(\mathrm{~s}, 1 \mathrm{H}), 2.98$ (q, $J$ $=7.6 \mathrm{~Hz}, 2 \mathrm{H}), 2.69(\mathrm{~s}, 3 \mathrm{H}), 1.39(\mathrm{t}, J=7.6 \mathrm{~Hz}, 3 \mathrm{H}) ;{ }^{13} \mathrm{C} \mathrm{NMR}\left(101 \mathrm{MHz}, \mathrm{CDCl}_{3}\right) \delta$ $163.65,147.55,144.44,129.21,129.06,126.76,125.42,123.56,121.52,32.15,18.69$, 14.05; HRMS (ESI) m/z calculated for $\mathrm{C}_{12} \mathrm{H}_{14} \mathrm{~N}\left[(\mathrm{M}+\mathrm{H})^{+}\right]$172.1121, found 172.1120; IR 
(film) $2967,2932,1603,1561,1506,1447,1410,977,923,861,754 \mathrm{~cm}^{-1}$. Spectra data are consistent with those reported in the literature: Synthesis 2017, 49, 188-194.

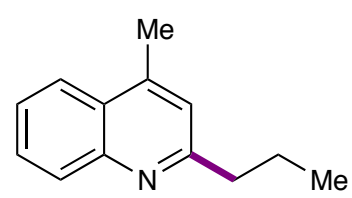

34

4-Methyl-2-propylquinoline (34): According to the general procedure $\mathrm{A}, \mathrm{FeSO}_{4} \cdot 7 \mathrm{H}_{2} \mathrm{O}$ (7.0 mg, $0.025 \mathrm{mmol}, 0.05$ equiv.), picolinic acid (6.2 mg, $0.05 \mathrm{mmol}, 0.1$ equiv.), lepidine (72.3 mg, $0.50 \mathrm{mmol}, 1.0$ equiv.), butyric acid (445.0 mg, $5.0 \mathrm{mmol}, 10$ equiv.), $\mathrm{NaBrO}_{3}\left(150.9 \mathrm{mg}, 1.0 \mathrm{mmol}, 2.0\right.$ equiv.), $4.5 \mathrm{~mL}$ of $\mathrm{H}_{2} \mathrm{O}$ and $0.5 \mathrm{~mL}$ of DMSO, $456 \mathrm{~nm}$ LEDs were used. After 24 hours, the reaction mixture was subjected to the workup procedure outlined in the general procedure and purified by flash chromatography $(10 \%$ ethyl acetate/hexanes) to provide the title compound as a colorless oil (72.1 mg, 78\% yield). ${ }^{1} \mathrm{H}$ NMR (400 MHz, $\left.\mathrm{CDCl}_{3}\right) \delta 8.05(\mathrm{~d}, J=8.4 \mathrm{~Hz}, 1 \mathrm{H}), 7.94(\mathrm{~d}, J=8.3 \mathrm{~Hz}, 1 \mathrm{H})$, $7.66(\mathrm{t}, J=7.6 \mathrm{~Hz}, 1 \mathrm{H}), 7.49$ (t, $J=7.6 \mathrm{~Hz}, 1 \mathrm{H}), 7.13(\mathrm{~s}, 1 \mathrm{H}), 2.90(\mathrm{t}, J=7.6 \mathrm{~Hz}, 2 \mathrm{H})$, $2.66(\mathrm{~s}, 3 \mathrm{H}), 1.83(\mathrm{~h}, J=7.4 \mathrm{~Hz}, 2 \mathrm{H}), 1.02(\mathrm{t}, J=7.4 \mathrm{~Hz}, 3 \mathrm{H}) ;{ }^{13} \mathrm{C} \mathrm{NMR}(101 \mathrm{MHz}$, $\left.\mathrm{CDCl}_{3}\right) \delta 162.48,147.59,144.14,129.21,128.96,126.72,125.34,123.51,122.03,41.10$, 23.24, 18.63, 14.00; HRMS (ESI) m/z calculated for $\mathrm{C}_{13} \mathrm{H}_{16} \mathrm{~N}\left[(\mathrm{M}+\mathrm{H})^{+}\right]$186.1277, found 186.1276; IR (film) 3061, 2959, 2870, 1603, 1560, 1508, 1482, 1379, 1357, $758 \mathrm{~cm}^{-1}$. Spectra data are consistent with those reported in the literature: Org. Lett. 2017, 19, 65946597. 


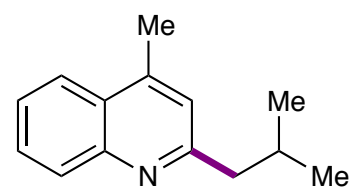

35

2-Isobutyl-4-methylquinoline (35): According to the general procedure $\mathrm{A}, \mathrm{FeSO}_{4} \cdot 7 \mathrm{H}_{2} \mathrm{O}$ (14.0 mg, $0.05 \mathrm{mmol}, 0.1$ equiv.), picolinic acid ( $12.4 \mathrm{mg}, 0.1 \mathrm{mmol}, 0.2$ equiv.), lepidine (72.3 mg, $0.50 \mathrm{mmol}, 1.0$ equiv.), 3-methylbutanoic acid (515.8 mg, $5.0 \mathrm{mmol}, 10$ equiv.), $\mathrm{NaBrO}_{3}$ (226.4 mg, $1.5 \mathrm{mmol}, 3.0$ equiv.), $4.0 \mathrm{~mL}$ of $\mathrm{H}_{2} \mathrm{O}$ and $1.0 \mathrm{~mL}$ of DMSO, $440 \mathrm{~nm}$ LEDs were used. After 24 hours, the reaction mixture was subjected to the workup procedure outlined in the general procedure and purified by flash chromatography $(10 \%$ ethyl acetate/hexanes) to provide the title compound as a colorless oil (60.5 mg, $61 \%$ yield). ${ }^{1} \mathrm{H} \mathrm{NMR}\left(400 \mathrm{MHz}, \mathrm{CDCl}_{3}\right) \delta 8.06(\mathrm{~d}, J=8.4 \mathrm{~Hz}, 1 \mathrm{H}), 7.94(\mathrm{~d}, J=8.3 \mathrm{~Hz}, 1 \mathrm{H})$, 7.66 (t, $J=7.6 \mathrm{~Hz}, 1 \mathrm{H}), 7.49$ (t, $J=7.5 \mathrm{~Hz}, 1 \mathrm{H}), 7.10(\mathrm{~s}, 1 \mathrm{H}), 2.79(\mathrm{~d}, J=7.4 \mathrm{~Hz}, 2 \mathrm{H})$, $2.66(\mathrm{~s}, 3 \mathrm{H}), 2.21$ (hept, $J=6.7 \mathrm{~Hz}, 1 \mathrm{H}), 0.97(\mathrm{~d}, J=6.6 \mathrm{~Hz}, 6 \mathrm{H}) ;{ }^{13} \mathrm{C} \mathrm{NMR}(101 \mathrm{MHz}$, $\left.\mathrm{CDCl}_{3}\right) \delta 161.80,147.58,143.89,129.24,128.92,126.69,125.33,123.50,122.65,48.09$, 29.33, 22.51, 18.62; HRMS (ESI) $\mathrm{m} / \mathrm{z}$ calculated for $\mathrm{C}_{14} \mathrm{H}_{18} \mathrm{~N}\left[(\mathrm{M}+\mathrm{H})^{+}\right] 200.1434$, found 200.1433; IR (film) 2953, 2927, 2867, 1601, 1560, 1508, 1463, 1447, 1125, 994, $753 \mathrm{~cm}^{-}$ 1. Spectra data are consistent with those reported in the literature: Org. Lett. 2017, 19, 6594-6597.<smiles>C=CCCc1cc(C)c2ccccc2n1</smiles> 
2-(But-3-en-1-yl)-4-methylquinoline (36): According to the general procedure B, $\mathrm{FeSO}_{4} \cdot 7 \mathrm{H}_{2} \mathrm{O}(14.0 \mathrm{mg}, 0.05 \mathrm{mmol}, 0.1$ equiv.), picolinic acid (12.4 mg, $0.1 \mathrm{mmol}, 0.2$ equiv.), lepidine (72.3 mg, $0.50 \mathrm{mmol}, 1.0$ equiv.), pent-4-enoic acid (505.7 mg, 5.0 mmol, 10 equiv.), $\mathrm{NaClO}_{3}$ (161.3 mg, $1.5 \mathrm{mmol}, 3.0$ equiv.), $4.0 \mathrm{~mL}$ of $\mathrm{H}_{2} \mathrm{O}$ and $1.0 \mathrm{~mL}$ of DMSO, $427 \mathrm{~nm}$ LEDs were used. After 24 hours, the reaction mixture was subjected to the workup procedure outlined in the general procedure and purified by flash chromatography (5\% ethyl acetate/hexanes) to provide the title compound as a yellow oil (44.7 mg, 45\% yield). ${ }^{1} \mathrm{H}$ NMR (400 MHz, $\left.\mathrm{CDCl}_{3}\right) \delta 8.04$ (d, $\left.J=8.4 \mathrm{~Hz}, 1 \mathrm{H}\right), 7.94$ (d, $J$ $=8.3 \mathrm{~Hz}, 1 \mathrm{H}), 7.67(\mathrm{ddd}, J=8.3,6.8,1.5 \mathrm{~Hz}, 1 \mathrm{H}), 7.49(\mathrm{ddd}, J=8.2,6.8,1.3 \mathrm{~Hz}, 1 \mathrm{H})$, 7.13 (s, 1H), 5.93 (ddt, $J=16.8,10.2,6.5 \mathrm{~Hz}, 1 \mathrm{H}), 5.09$ (dd, $J=17.1,1.7 \mathrm{~Hz}, 1 \mathrm{H}), 4.99$ $(\mathrm{dd}, J=10.2,1.7 \mathrm{~Hz}, 1 \mathrm{H}), 3.02(\mathrm{t}, J=8.2 \mathrm{~Hz}, 2 \mathrm{H}), 2.66(\mathrm{~s}, 3 \mathrm{H}), 2.61-2.54(\mathrm{~m}, 2 \mathrm{H}) ;{ }^{13} \mathrm{C}$ NMR (101 MHz, $\left.\mathrm{CDCl}_{3}\right) \delta 161.61,147.65,144.23,137.74,129.25,129.02,126.77$, 125.45, 123.54, 122.05, 115.08, 38.36, 33.78, 18.64; HRMS (ESI) m/z calculated for $\mathrm{C}_{14} \mathrm{H}_{16} \mathrm{~N}\left[(\mathrm{M}+\mathrm{H})^{+}\right]$198.1277, found 198.1276; IR (film) 2975, 2921, 1640, 1602, 1561, $1508,1446,1411,910,861,755 \mathrm{~cm}^{-1}$. Spectra data are consistent with those reported in the literature: Synthesis 2017, 49, 188-194.

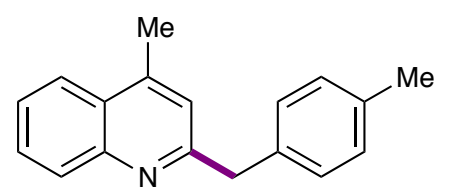

37

4-Methyl-2-(4-methylbenzyl)quinoline (37): According to the general procedure B, $\mathrm{FeSO}_{4} \cdot 7 \mathrm{H}_{2} \mathrm{O}(14.0 \mathrm{mg}, 0.05 \mathrm{mmol}, 0.1$ equiv.), picolinic acid ( $12.4 \mathrm{mg}, 0.1 \mathrm{mmol}, 0.2$ equiv.), lepidine (72.3 mg, $0.50 \mathrm{mmol}, 1.0$ equiv.), 2-(p-tolyl)acetic acid (379.2 mg, 2.5 mmol, 5.0 equiv.), $\mathrm{NaClO}_{3}\left(161.3 \mathrm{mg}, 1.5 \mathrm{mmol}, 3.0\right.$ equiv.), $4.0 \mathrm{~mL}$ of $\mathrm{H}_{2} \mathrm{O}$ and $1.0 \mathrm{~mL}$ 
of DMSO, 440 nm LEDs were used. After 24 hours, the reaction mixture was subjected to the workup procedure outlined in the general procedure and purified by flash chromatography (5\% ethyl acetate/hexanes) to provide the title compound as a white solid (104.2 mg, 84\% yield). ${ }^{1} \mathrm{H}$ NMR (400 MHz, $\left.\mathrm{CDCl}_{3}\right) \delta 8.13(\mathrm{~d}, J=8.3 \mathrm{~Hz}, 1 \mathrm{H}), 7.93$ $(\mathrm{d}, J=8.3 \mathrm{~Hz}, 1 \mathrm{H}), 7.70(\mathrm{t}, J=7.6 \mathrm{~Hz}, 1 \mathrm{H}), 7.52$ (t, $J=7.6 \mathrm{~Hz}, 1 \mathrm{H}), 7.24(\mathrm{~d}, J=7.7 \mathrm{~Hz}$ 2H), $7.13(\mathrm{~d}, J=7.5 \mathrm{~Hz}, 2 \mathrm{H}), 7.07(\mathrm{~s}, 1 \mathrm{H}), 4.28(\mathrm{~s}, 2 \mathrm{H}), 2.59(\mathrm{~s}, 3 \mathrm{H}), 2.34(\mathrm{~s}, 3 \mathrm{H}) ;{ }^{13} \mathrm{C}$ NMR $\left(101 \mathrm{MHz} \mathrm{CDCl}_{3}\right) \delta 161.01,147.49,144.42,136.18,135.84,129.38,129.19$, 129.02, 128.98, 126.75, 125.57, 123.51, 122.02, 45.00, 20.95, 18.58; HRMS (ESI) m/z calculated for $\mathrm{C}_{18} \mathrm{H}_{18} \mathrm{~N}\left[(\mathrm{M}+\mathrm{H})^{+}\right]$248.1434, found 248.1433; IR (film) 2949, 2918, 2854, $1599,1444,1353,1153,1022,806,786,752 \mathrm{~cm}^{-1}$.

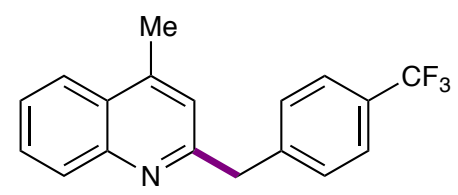

38

4-Methyl-2-(4-(trifluoromethyl)benzyl)quinoline (38): According to the general procedure $\mathrm{B}, \mathrm{FeSO}_{4} \cdot 7 \mathrm{H}_{2} \mathrm{O}$ (14.0 $\mathrm{mg}, 0.05 \mathrm{mmol}, 0.1$ equiv.), picolinic acid (12.4 $\mathrm{mg}, 0.1$ mmol, 0.2 equiv.), lepidine ( $72.3 \mathrm{mg}, 0.50 \mathrm{mmol}, 1.0$ equiv.), 2-(4(trifluoromethyl)phenyl)acetic acid (520.8 mg, 2.5 mmol, 5.0 equiv.), $\mathrm{NaClO}_{3}$ (161.3 mg, $1.5 \mathrm{mmol}, 3.0$ equiv.), $4.0 \mathrm{~mL}$ of $\mathrm{H}_{2} \mathrm{O}$ and $1.0 \mathrm{~mL}$ of DMSO, $440 \mathrm{~nm}$ LEDs were used. After 24 hours, the reaction mixture was subjected to the workup procedure outlined in the general procedure and purified by flash chromatography (10\% ethyl acetate/hexanes) to provide the title compound as a light yellow oil (97.1 mg, 65\% yield). ${ }^{1} \mathrm{H}$ NMR (400 $\left.\mathrm{MHz}, \mathrm{CDCl}_{3}\right) \delta 8.09(\mathrm{~d}, J=8.4 \mathrm{~Hz}, 1 \mathrm{H}), 7.95(\mathrm{~d}, J=8.3 \mathrm{~Hz}, 1 \mathrm{H}), 7.71(\mathrm{ddd}, J=8.4,6.8$, $1.4 \mathrm{~Hz}, 1 \mathrm{H}), 7.58-7.51(\mathrm{~m}, 3 \mathrm{H}), 7.43(\mathrm{~d}, J=8.1 \mathrm{~Hz}, 2 \mathrm{H}), 7.05(\mathrm{~s}, 1 \mathrm{H}), 4.34(\mathrm{~s}, 2 \mathrm{H})$, 
2.63 (s, 3H); ${ }^{13} \mathrm{C}$ NMR (101 MHz, $\left.\mathrm{CDCl}_{3}\right) \delta 159.61,147.52,145.16,143.33$ (d), 129.41

(2), 129.38, 128.75 (q), 126.88, 126.02, 125.47 (q), 124.22 (q), 123.65, 122.04, 45.02, 18.68; HRMS (ESI) $\mathrm{m} / \mathrm{z}$ calculated for $\mathrm{C}_{18} \mathrm{H}_{15} \mathrm{~F}_{3} \mathrm{~N}\left[(\mathrm{M}+\mathrm{H})^{+}\right]$302.1151, found 302.1150; IR (film) 2953, 2924, 1601, 1561, 1509, 1417, 1312, 1118, 1065, 1018, $755 \mathrm{~cm}^{-1}$.

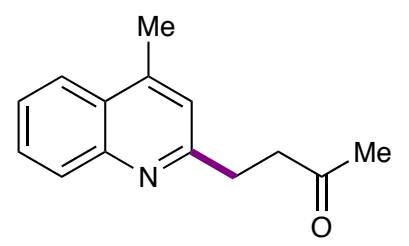

39

4-(4-Methylquinolin-2-yl)butan-2-one (39): According to the general procedure B, $\mathrm{FeSO}_{4} \cdot 7 \mathrm{H}_{2} \mathrm{O}(14.0 \mathrm{mg}, 0.05 \mathrm{mmol}, 0.1$ equiv.), picolinic acid ( $12.4 \mathrm{mg}, 0.1 \mathrm{mmol}, 0.2$ equiv.), lepidine (72.3 mg, $0.50 \mathrm{mmol}, 1.0$ equiv.), 4-oxopentanoic acid (586.5 mg, 5.0 mmol, 10 equiv.), $\mathrm{NaClO}_{3}$ (161.3 mg, $1.5 \mathrm{mmol}, 3.0$ equiv.), $4.0 \mathrm{~mL}$ of $\mathrm{H}_{2} \mathrm{O}$ and $1.0 \mathrm{~mL}$ of DMSO, $427 \mathrm{~nm}$ LEDs were used. After 24 hours, the reaction mixture was subjected to the workup procedure outlined in the general procedure and purified by flash chromatography (20\% ethyl acetate/hexanes) to provide the title compound as a colorless oil (48.5 mg, 46\% yield). ${ }^{1} \mathrm{H}$ NMR (400 MHz, $\left.\mathrm{CDCl}_{3}\right) \delta 7.99(\mathrm{~d}, J=8.4 \mathrm{~Hz}, 1 \mathrm{H}), 7.94(\mathrm{~d}$, $J=8.3 \mathrm{~Hz}, 1 \mathrm{H}), 7.66(\mathrm{t}, J=7.6 \mathrm{~Hz}, 1 \mathrm{H}), 7.50$ (t, $J=7.6 \mathrm{~Hz}, 1 \mathrm{H}), 7.16(\mathrm{~s}, 1 \mathrm{H}), 3.20$ (t, $J$ $=7.1 \mathrm{~Hz}, 2 \mathrm{H}), 3.04(\mathrm{t}, J=7.2 \mathrm{~Hz}, 2 \mathrm{H}), 2.66(\mathrm{~s}, 3 \mathrm{H}), 2.23(\mathrm{~s}, 3 \mathrm{H}) ;{ }^{13} \mathrm{C} \mathrm{NMR}(101 \mathrm{MHz}$, $\left.\mathrm{CDCl}_{3}\right) \delta 208.15,160.46,147.46,144.40,129.09,129.05,126.82,125.55,123.59,122.37$, 42.06, 32.19, 30.13, 18.59; HRMS (ESI) m/z calculated for $\mathrm{C}_{14} \mathrm{H}_{16} \mathrm{NO}\left[(\mathrm{M}+\mathrm{H})^{+}\right]$ 214.1226, found 214.1226; IR (film) 2908, 1712, 1603, 1561, 1507, 1446, 1409, 1356, $1159,860,755 \mathrm{~cm}^{-1}$. 


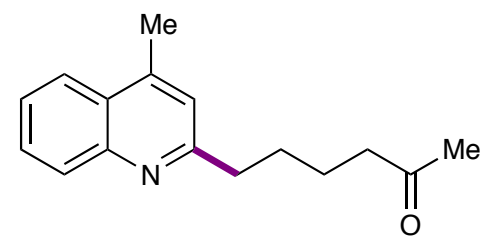

40

6-(4-Methylquinolin-2-yl)hexan-2-one (40): According to the general procedure B, $\mathrm{FeSO}_{4} \cdot 7 \mathrm{H}_{2} \mathrm{O}$ (14.0 mg, $0.05 \mathrm{mmol}, 0.1$ equiv.), picolinic acid (12.4 mg, $0.1 \mathrm{mmol}, 0.2$ equiv.), lepidine (72.3 mg, $0.50 \mathrm{mmol}, 1.0$ equiv.), 6-oxoheptanoic acid (726.7 mg, 5.0 mmol, 10 equiv.), $\mathrm{NaClO}_{3}$ (161.3 mg, $1.5 \mathrm{mmol}, 3.0$ equiv.), $4.5 \mathrm{~mL}$ of $\mathrm{H}_{2} \mathrm{O}$ and $0.5 \mathrm{~mL}$ of DMSO, $440 \mathrm{~nm}$ LEDs were used. After 24 hours, the reaction mixture was subjected to the workup procedure outlined in the general procedure and purified by flash chromatography ( $20 \%$ ethyl acetate/hexanes) to provide the title compound as a colorless oil (84.6 mg, 70\% yield). ${ }^{1} \mathrm{H}$ NMR (400 MHz, $\left.\mathrm{CDCl}_{3}\right) \delta 8.01(\mathrm{~d}, J=8.4 \mathrm{~Hz}, 1 \mathrm{H}), 7.92$ $(\mathrm{dd}, J=8.3,1.6 \mathrm{~Hz}, 1 \mathrm{H}), 7.65(\mathrm{ddd}, J=8.4,6.9,1.5 \mathrm{~Hz}, 1 \mathrm{H}), 7.48(\mathrm{ddd}, J=8.2,6.9,1.3$ $\mathrm{Hz}, 1 \mathrm{H}), 7.11(\mathrm{~s}, 1 \mathrm{H}), 2.91(\mathrm{t}, J=7.5 \mathrm{~Hz}, 2 \mathrm{H}), 2.64(\mathrm{~s}, 3 \mathrm{H}), 2.47(\mathrm{t}, J=7.3 \mathrm{~Hz}, 2 \mathrm{H}), 2.10$ (s, 3H), $1.85-1.75(\mathrm{~m}, 2 \mathrm{H}), 1.72-1.63(\mathrm{~m}, 2 \mathrm{H}) ;{ }^{13} \mathrm{C} \mathrm{NMR}\left(101 \mathrm{MHz}, \mathrm{CDCl}_{3}\right) \delta 208.96$, $161.95,147.59,144.34,129.20,129.04,126.75,125.45,123.56,121.99,43.47,38.78$, 29.85, 29.26, 23.57, 18.64; HRMS (ESI) m/z calculated for $\mathrm{C}_{16} \mathrm{H}_{20} \mathrm{NO}\left[(\mathrm{M}+\mathrm{H})^{+}\right]$ 242.1539, found 242.1539; IR (film) 2934, 2860, 1710, 1602, 1561, 1507, 1447, 1356, $1160,863,757 \mathrm{~cm}^{-1}$.<smiles>CCOC(=O)CCCc1cc(C)c2ccccc2n1</smiles> 
Ethyl 4-(4-methylquinolin-2-yl)butanoate (41): According to the general procedure B, $\mathrm{FeSO}_{4} \cdot 7 \mathrm{H}_{2} \mathrm{O}$ (14.0 mg, $0.05 \mathrm{mmol}, 0.1$ equiv.), picolinic acid (12.4 mg, $0.1 \mathrm{mmol}, 0.2$ equiv.), lepidine (72.3 mg, $0.50 \mathrm{mmol}, 1.0$ equiv.), 5-ethoxy-5-oxopentanoic acid (817.2 mg, 5.0 mmol, 10 equiv.), $\mathrm{NaClO}_{3}$ (161.3 mg, $1.5 \mathrm{mmol}, 3.0$ equiv.), $4.0 \mathrm{~mL}$ of $\mathrm{H}_{2} \mathrm{O}$ and $1.0 \mathrm{~mL}$ of DMSO, $440 \mathrm{~nm}$ LEDs were used. After 24 hours, the reaction mixture was subjected to the workup procedure outlined in the general procedure and purified by flash chromatography (33\% ethyl acetate/hexanes) to provide the title compound as a colorless oil (70.9 mg, 55\% yield). ${ }^{1} \mathrm{H}$ NMR (400 MHz, $\left.\mathrm{CDCl}_{3}\right) \delta 8.03(\mathrm{~d}, J=8.4 \mathrm{~Hz}, 1 \mathrm{H}), 7.95$ (d, $J=8.2 \mathrm{~Hz}, 1 \mathrm{H}), 7.67$ (t, $J=7.6 \mathrm{~Hz}, 1 \mathrm{H}), 7.51(\mathrm{t}, J=7.3 \mathrm{~Hz}, 1 \mathrm{H}), 7.15(\mathrm{~s}, 1 \mathrm{H}), 4.12(\mathrm{q}, J$ $=7.1 \mathrm{~Hz}, 2 \mathrm{H}), 2.97(\mathrm{t}, J=7.7 \mathrm{~Hz}, 2 \mathrm{H}), 2.68(\mathrm{~s}, 3 \mathrm{H}), 2.41(\mathrm{t}, J=7.5 \mathrm{~Hz}, 2 \mathrm{H}), 2.15(\mathrm{p}, J=$ $7.6 \mathrm{~Hz}, 2 \mathrm{H}), 1.24(\mathrm{t}, J=7.1 \mathrm{~Hz}, 3 \mathrm{H}) ;{ }^{13} \mathrm{C} \mathrm{NMR}\left(101 \mathrm{MHz}, \mathrm{CDCl}_{3}\right) \delta$ 173.40, 161.30, $147.42,144.65,129.14,129.09,126.78,125.59,123.55,122.01,60.26,37.96,33.74$, 24.90, 18.64, 14.16; HRMS (ESI) m/z calculated for $\mathrm{C}_{16} \mathrm{H}_{20} \mathrm{NO}_{2}\left[(\mathrm{M}+\mathrm{H})^{+}\right] 258.1489$, found 258.1488; IR (film) 2977, 2933, 1728, 1602, 1446, 1243, 1178, 1145, 1025, 800, $756 \mathrm{~cm}^{-1}$.

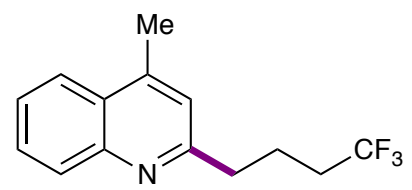

42

4-Methyl-2-(4,4,4-trifluorobutyl)quinoline (42): According to the general procedure A, $\mathrm{FeSO}_{4} \cdot 7 \mathrm{H}_{2} \mathrm{O}$ (14.0 mg, $0.05 \mathrm{mmol}, 0.1$ equiv.), picolinic acid (12.4 mg, $0.1 \mathrm{mmol}, 0.2$ equiv.), lepidine (72.3 mg, $0.50 \mathrm{mmol}, 1.0$ equiv.), 5,5,5-trifluoropentanoic acid (781.7 mg, 5.0 mmol, 10 equiv.), $\mathrm{NaBrO}_{3}\left(226.4 \mathrm{mg}, 1.5 \mathrm{mmol}, 3.0\right.$ equiv.), $4.0 \mathrm{~mL}$ of $\mathrm{H}_{2} \mathrm{O}$ and $1.0 \mathrm{~mL}$ of DMSO, $440 \mathrm{~nm}$ LEDs were used. After 24 hours, the reaction mixture was 
subjected to the workup procedure outlined in the general procedure and purified by flash chromatography ( $10 \%$ ethyl acetate/hexanes) to provide the title compound as a colorless oil (38.3 mg, 30\% yield). ${ }^{1} \mathrm{H}$ NMR (400 MHz, $\left.\mathrm{CDCl}_{3}\right) \delta 8.04(\mathrm{~d}, J=8.4 \mathrm{~Hz}, 1 \mathrm{H}), 7.96(\mathrm{~d}$, $J=8.3 \mathrm{~Hz}, 1 \mathrm{H}), 7.69(\mathrm{t}, J=7.6 \mathrm{~Hz}, 1 \mathrm{H}), 7.53$ (t, $J=7.6 \mathrm{~Hz}, 1 \mathrm{H}), 7.14$ (s, 1H), 3.00 (t, $J$ $=7.4 \mathrm{~Hz}, 2 \mathrm{H}), 2.69(\mathrm{~s}, 3 \mathrm{H}), 2.27-2.15(\mathrm{~m}, 2 \mathrm{H}), 2.15-2.06(\mathrm{~m}, 2 \mathrm{H}) ;{ }^{13} \mathrm{C} \mathrm{NMR}(101$ $\left.\mathrm{MHz}, \mathrm{CDCl}_{3}\right) \delta 160.50,147.54,144.87,129.29,129.23,127.11$ (q), 126.86, 125.77, 123.62, 121.84, 37.43, 33.27 (q), 21.83 (q), 18.67; HRMS (ESI) m/z calculated for $\mathrm{C}_{14} \mathrm{H}_{15} \mathrm{~F}_{3} \mathrm{~N}\left[(\mathrm{M}+\mathrm{H})^{+}\right]$254.1151, found 254.1150; IR (film) 2946, 1603, 1412, 1386, 1524, $1208,1129,1105,1067,1015,756 \mathrm{~cm}^{-1}$.

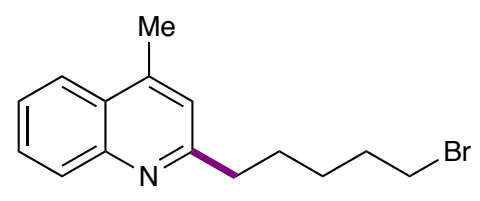

43

2-(5-Bromopentyl)-4-methylquinoline (43): According to the general procedure A, $\mathrm{FeSO}_{4} \cdot 7 \mathrm{H}_{2} \mathrm{O}(14.0 \mathrm{mg}, 0.05 \mathrm{mmol}, 0.1$ equiv.), picolinic acid ( $12.4 \mathrm{mg}, 0.1 \mathrm{mmol}, 0.2$ equiv.), lepidine (72.3 mg, $0.50 \mathrm{mmol}, 1.0$ equiv.), 6-bromohexanoic acid (1005.4 mg, $5.0 \mathrm{mmol}, 10$ equiv.), $\mathrm{NaBrO}_{3}$ (226.4 mg, $1.5 \mathrm{mmol}, 3.0$ equiv.), $4.0 \mathrm{~mL}$ of $\mathrm{H}_{2} \mathrm{O}$ and 1.0 $\mathrm{mL}$ of DMSO, $427 \mathrm{~nm}$ LEDs were used. After 24 hours, the reaction mixture was subjected to the workup procedure outlined in the general procedure and purified by flash chromatography ( $10 \%$ ethyl acetate/hexanes) to provide the title compound as a yellow solid (76.5 mg, 53\% yield). ${ }^{1} \mathrm{H}$ NMR (400 MHz, $\left.\mathrm{CDCl}_{3}\right) \delta 8.04(\mathrm{~d}, J=8.4 \mathrm{~Hz}, 1 \mathrm{H}), 7.95$ (dd, $J=8.4,1.2 \mathrm{~Hz}, 1 \mathrm{H}), 7.67$ (ddd, $J=8.3,6.8,1.3 \mathrm{~Hz}, 1 \mathrm{H}), 7.50$ (ddd, $J=8.2,6.8,1.2$ Hz, 1H), 7.13 (s, 1H), 3.41 (t, $J=6.8 \mathrm{~Hz}, 2 \mathrm{H}), 2.93$ (t, $J=7.8 \mathrm{~Hz}, 2 \mathrm{H}), 2.67$ (s, 3H), 1.92 (p, $J=7.8 \mathrm{~Hz}, 2 \mathrm{H}), 1.84$ (p, $J=7.8 \mathrm{~Hz}, 2 \mathrm{H}), 1.56(\mathrm{p}, J=7.6 \mathrm{~Hz}, 2 \mathrm{H}) ;{ }^{13} \mathrm{C}$ NMR $(101$ 
$\left.\mathrm{MHz}, \mathrm{CDCl}_{3}\right) \delta 162.07,147.56,144.47,129.20,129.13,126.78,125.52,123.60,122.01$, 38.83, 33.74, 32.64, 29.03, 28.04, 18.70; HRMS (ESI) m/z calculated for $\mathrm{C}_{15} \mathrm{H}_{19} \mathrm{BrN}$ $\left[(\mathrm{M}+\mathrm{H})^{+}\right]$292.0695, found 292.0695; IR (film) 2923, 2856, 1602, 1561, 1507, 1446, $1244,1129,1020,862,756 \mathrm{~cm}^{-1}$. Spectra data are consistent with those reported in the literature: J. Org. Chem. 2018, 83, 3000-3012.

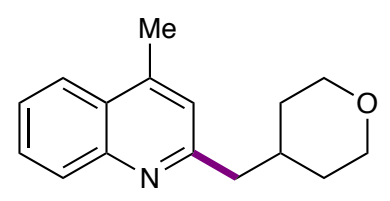

44

4-Methyl-2-((tetrahydro-2H-pyran-4-yl)methyl)quinoline (44): According to the general procedure $\mathrm{A}, \mathrm{FeSO}_{4} \cdot 7 \mathrm{H}_{2} \mathrm{O}$ ( $7.0 \mathrm{mg}, 0.025 \mathrm{mmol}, 0.05$ equiv.), picolinic acid (6.2 $\mathrm{mg}, 0.05 \mathrm{mmol}, 0.1$ equiv.), lepidine ( $72.3 \mathrm{mg}, 0.50 \mathrm{mmol}, 1.0$ equiv.), 2-(tetrahydro- $2 \mathrm{H}$ pyran-4-yl)acetic acid (743.1 mg, $5.0 \mathrm{mmol}, 10$ equiv.), $\mathrm{NaBrO}_{3}(150.9 \mathrm{mg}, 1.0 \mathrm{mmol}$, 2.0 equiv.), $4.0 \mathrm{~mL}$ of $\mathrm{H}_{2} \mathrm{O}$ and $1.0 \mathrm{~mL}$ of DMSO, $440 \mathrm{~nm}$ LEDs were used. After 24 hours, the reaction mixture was subjected to the workup procedure outlined in the general procedure and purified by flash chromatography ( $50 \%$ ethyl acetate/hexanes) to provide the title compound as a white solid ( $80.5 \mathrm{mg}, 67 \%$ yield). ${ }^{1} \mathrm{H} \mathrm{NMR}\left(400 \mathrm{MHz}, \mathrm{CDCl}_{3}\right) \delta$ $8.06(\mathrm{~d}, J=8.4 \mathrm{~Hz}, 1 \mathrm{H}), 7.95(\mathrm{~d}, J=8.3 \mathrm{~Hz}, 1 \mathrm{H}), 7.67(\mathrm{t}, J=7.6 \mathrm{~Hz}, 1 \mathrm{H}), 7.50$ (t, $J=7.5$ Hz, 1H), 7.10 (s, 1H), 3.93 (dd, $J=11.8,4.4 \mathrm{~Hz}, 2 \mathrm{H}), 3.35$ (t, $J=11.4 \mathrm{~Hz}, 2 \mathrm{H}), 2.86(\mathrm{~d}, J$ $=7.3 \mathrm{~Hz}, 2 \mathrm{H}), 2.67(\mathrm{~s}, 3 \mathrm{H}), 2.19-2.05(\mathrm{~m}, 1 \mathrm{H}), 1.64-1.52(\mathrm{~m}, 2 \mathrm{H}), 1.45(\mathrm{qd}, J=12.1$, $4.5 \mathrm{~Hz}, 2 \mathrm{H}) ;{ }^{13} \mathrm{C}$ NMR $\left(101 \mathrm{MHz}, \mathrm{cdcl}_{3}\right) \delta 160.29,147.48,144.15,129.10,129.05$, 126.65, 125.48, 123.49, 122.69, 67.81, 45.99, 35.86, 32.90, 18.58; HRMS (ESI) m/z calculated for $\mathrm{C}_{16} \mathrm{H}_{20} \mathrm{NO}\left[(\mathrm{M}+\mathrm{H})^{+}\right]$242.1539, found 242.1538; IR (film) 2915, 2839, 
$1601,1443,1132,1089,1013,982,844,801,756 \mathrm{~cm}^{-1}$. Spectra data are consistent with those reported in the literature: Angew. Chem. Int. Ed. 2017, 56, 15309-15313.

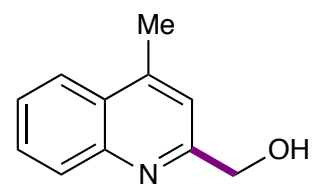

45

(4-Methylquinolin-2-yl)methanol (45): According to the general procedure B,

$\mathrm{FeSO}_{4} \cdot 7 \mathrm{H}_{2} \mathrm{O}$ (14.0 mg, $0.05 \mathrm{mmol}, 0.1$ equiv.), picolinic acid (12.4 mg, $0.1 \mathrm{mmol}, 0.2$ equiv.), lepidine (72.3 mg, $0.50 \mathrm{mmol}, 1.0$ equiv.), 2-hydroxyacetic acid (384.1 mg, 5.0 mmol, 10 equiv.), $\mathrm{NaClO}_{3}$ (161.3 mg, $1.5 \mathrm{mmol}, 3.0$ equiv.), $4.0 \mathrm{~mL}$ of $\mathrm{H}_{2} \mathrm{O}$ and $1.0 \mathrm{~mL}$ of DMSO, $440 \mathrm{~nm}$ LEDs were used. After 24 hours, the reaction mixture was subjected to the workup procedure outlined in the general procedure and purified by flash chromatography (50\% ethyl acetate/hexanes) to provide the title compound as a white solid (36.6 mg, 42\% yield). ${ }^{1} \mathrm{H}$ NMR (400 MHz, $\left.\mathrm{CDCl}_{3}\right) \delta 8.06(\mathrm{~d}, J=8.4 \mathrm{~Hz}, 1 \mathrm{H}), 7.96$ $(\mathrm{d}, J=8.3 \mathrm{~Hz}, 1 \mathrm{H}), 7.70$ (ddd, $J=8.4,6.8,1.4 \mathrm{~Hz}, 1 \mathrm{H}), 7.55$ (ddd, $J=8.2,6.8,1.3 \mathrm{~Hz}$, 1H), $7.12(\mathrm{~s}, 1 \mathrm{H}), 4.87$ (s, 2H), $4.23($ br s, $1 \mathrm{H}), 2.68(\mathrm{~s}, 3 \mathrm{H}) ;{ }^{13} \mathrm{C} \mathrm{NMR}\left(101 \mathrm{MHz}, \mathrm{CDCl}_{3}\right)$ $\delta 158.65,146.42,145.08,129.39,129.04,127.55,126.03,123.75,118.93,63.98,18.74 ;$ HRMS (ESI) m/z calculated for $\mathrm{C}_{11} \mathrm{H}_{12} \mathrm{NO}\left[(\mathrm{M}+\mathrm{H})^{+}\right]$174.0913, found 174.0912; IR (film) $3145,2921,2825,1598,1566,1510,1447,1059,1035,866,753 \mathrm{~cm}^{-1}$. Spectra data are consistent with those reported in the literature: Org. Lett. 2018, 20, 3229-3232.<smiles>O=[N+]([O-])c1cc(COc2ccccc2)nc2ccccc12</smiles> 
4-Methyl-2-(phenoxymethyl)quinoline (46): According to the general procedure A, $\mathrm{FeSO}_{4} \cdot 7 \mathrm{H}_{2} \mathrm{O}$ (7.0 mg, $0.025 \mathrm{mmol}, 0.05$ equiv.), picolinic acid (6.2 mg, $0.05 \mathrm{mmol}, 0.1$ equiv.), lepidine (72.3 mg, $0.50 \mathrm{mmol}, 1.0$ equiv.), 2-phenoxyacetic acid (768.4 mg, 5.0 mmol, 10 equiv.), $\mathrm{NaBrO}_{3}$ (150.9 mg, $1.0 \mathrm{mmol}, 2.0$ equiv.), $4.5 \mathrm{~mL}$ of $\mathrm{H}_{2} \mathrm{O}$ and $0.5 \mathrm{~mL}$ of DMSO, $456 \mathrm{~nm}$ LEDs were used. After 8 hours, the reaction mixture was subjected to the workup procedure outlined in the general procedure and purified by flash chromatography (10\% ethyl acetate/hexanes) to provide the title compound as a yellow oil (100.0 mg, 80\% yield). ${ }^{1} \mathrm{H}$ NMR $\left(400 \mathrm{MHz}, \mathrm{CDCl}_{3}\right) \delta 8.09(\mathrm{~d}, J=8.4 \mathrm{~Hz}, 1 \mathrm{H}), 8.00$ $(\mathrm{d}, J=8.2 \mathrm{~Hz}, 1 \mathrm{H}), 7.73(\mathrm{ddd}, J=8.4,6.8,1.4 \mathrm{~Hz}, 1 \mathrm{H}), 7.57(\mathrm{ddd}, J=8.3,6.8,1.3 \mathrm{~Hz}$, 1H), $7.53(\mathrm{~s}, 1 \mathrm{H}), 7.33-7.27(\mathrm{~m}, 2 \mathrm{H}), 7.05(\mathrm{~d}, J=8.0 \mathrm{~Hz}, 2 \mathrm{H}), 6.97(\mathrm{t}, J=7.3 \mathrm{~Hz}, 1 \mathrm{H})$, $5.35(\mathrm{~s}, 2 \mathrm{H}), 2.72(\mathrm{~s}, 3 \mathrm{H}) ;{ }^{13} \mathrm{C} \mathrm{NMR}\left(101 \mathrm{MHz}, \mathrm{cdcl}_{3}\right) \delta 158.45,157.51,147.26,145.41$ $129.53,129.43,129.39,127.59,126.23,123.78,121.11,119.72,114.83,71.18,18.91$; HRMS (ESI) m/z calculated for $\mathrm{C}_{17} \mathrm{H}_{16} \mathrm{NO}\left[(\mathrm{M}+\mathrm{H})^{+}\right]$250.1226, found 250.1226; IR (film) $3060,3040,2923,1599,1495,1448,1242,1215,1172,1055,754 \mathrm{~cm}^{-1}$.

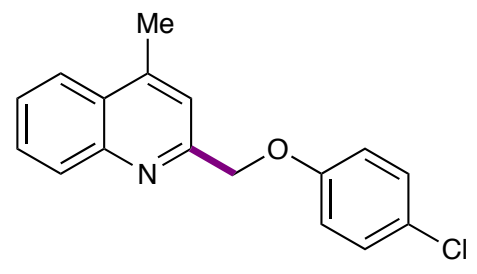

47

2-((4-Chlorophenoxy)methyl)-4-methylquinoline (47): According to the general procedure $\mathrm{B}, \mathrm{FeSO}_{4} \cdot 7 \mathrm{H}_{2} \mathrm{O}$ (14.0 mg, $0.05 \mathrm{mmol}, 0.1$ equiv.), picolinic acid (12.4 mg, 0.1 mmol, 0.2 equiv.), lepidine (72.3 $\mathrm{mg}, 0.50 \mathrm{mmol}, 1.0$ equiv.), 2-(4-chlorophenoxy)acetic acid (952.0 mg, 5.0 mmol, 10 equiv.), $\mathrm{NaClO}_{3}$ (161.3 mg, $1.5 \mathrm{mmol}, 3.0$ equiv.), $4.0 \mathrm{~mL}$ of $\mathrm{H}_{2} \mathrm{O}$ and $1.0 \mathrm{~mL}$ of DMSO, $440 \mathrm{~nm}$ LEDs were used. After 24 hours, the reaction 
mixture was subjected to the workup procedure outlined in the general procedure and purified by flash chromatography (10\% ethyl acetate/hexanes) to provide the title compound as a white solid (126.1 mg, 89\% yield). ${ }^{1} \mathrm{H}$ NMR (400 MHz, $\left.\mathrm{CDCl}_{3}\right) \delta 8.08(\mathrm{~d}$, $J=8.4 \mathrm{~Hz}, 1 \mathrm{H}), 7.99(\mathrm{~d}, J=8.3 \mathrm{~Hz}, 1 \mathrm{H}), 7.73(\mathrm{ddd}, J=8.4,6.8,1.4 \mathrm{~Hz}, 1 \mathrm{H}), 7.57$ (ddd, $J=8.2,6.8,1.3 \mathrm{~Hz}, 1 \mathrm{H}), 7.47(\mathrm{~s}, 1 \mathrm{H}), 7.25-7.20(\mathrm{~m}, 2 \mathrm{H}), 6.98-6.93(\mathrm{~m}, 2 \mathrm{H}), 5.30(\mathrm{~s}$, 2H), $2.71(\mathrm{~s}, 3 \mathrm{H}) ;{ }^{13} \mathrm{C} \mathrm{NMR}\left(101 \mathrm{MHz}, \mathrm{CDCl}_{3}\right) \delta 157.03,156.93,147.25,145.53,129.52$, $129.40,129.39,127.60,126.36,126.00,123.79,119.66,116.13,71.52,18.91$; HRMS (ESI) $\mathrm{m} / \mathrm{z}$ calculated for $\mathrm{C}_{17} \mathrm{H}_{15} \mathrm{ClNO}\left[(\mathrm{M}+\mathrm{H})^{+}\right] 284.0837$, found 284.0835; IR (film) $2913,2851,1598,1488,1447,1280,1244,1166,1060,817,750 \mathrm{~cm}^{-1}$.

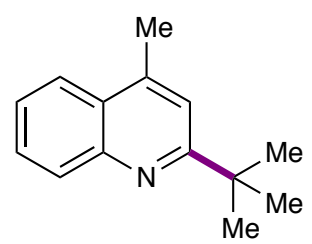

48

2-(tert-Butyl)-4-methylquinoline (48): According to the general procedure A, $\mathrm{FeSO}_{4} \cdot 7 \mathrm{H}_{2} \mathrm{O}(7.0 \mathrm{mg}, 0.025 \mathrm{mmol}, 0.05$ equiv.), picolinic acid (6.2 mg, $0.05 \mathrm{mmol}, 0.1$ equiv.), lepidine (72.3 mg, $0.50 \mathrm{mmol}, 1.0$ equiv.), pivalic acid (515.8 mg, $5.0 \mathrm{mmol}, 10$ equiv.), $\mathrm{NaBrO}_{3}$ (226.4 mg, $1.5 \mathrm{mmol}, 3.0$ equiv.), $4.0 \mathrm{~mL}$ of $\mathrm{H}_{2} \mathrm{O}$ and $1.0 \mathrm{~mL}$ of DMSO, $440 \mathrm{~nm}$ LEDs were used. After 24 hours, the reaction mixture was subjected to the workup procedure outlined in the general procedure and purified by flash chromatography ( $5 \%$ ethyl acetate/hexanes) to provide the title compound as a colorless oil (63.5 mg, 64\% yield). ${ }^{1} \mathrm{H}$ NMR (400 MHz, $\left.\mathrm{CDCl}_{3}\right) \delta 8.15(\mathrm{~d}, J=8.4 \mathrm{~Hz}, 1 \mathrm{H}), 7.96$ $(\mathrm{dd}, J=8.4,1.2 \mathrm{~Hz}, 1 \mathrm{H}), 7.70$ (ddd, $J=8.3,7.0,1.3 \mathrm{~Hz}, 1 \mathrm{H}), 7.52$ (ddd, $J=8.1,7.0,1.1$ $\mathrm{Hz}, 1 \mathrm{H}), 7.41(\mathrm{~s}, 1 \mathrm{H}), 2.71(\mathrm{~s}, 3 \mathrm{H}), 1.54(\mathrm{~s}, 9 \mathrm{H}) ;{ }^{13} \mathrm{C} \mathrm{NMR}\left(101 \mathrm{MHz}, \mathrm{CDCl}_{3}\right) \delta 168.79$, 
$147.23,143.51,129.88,128.60,126.46,125.30,123.28,118.78,37.83,30.07,18.84$;

HRMS (ESI) m/z calculated for $\mathrm{C}_{14} \mathrm{H}_{18} \mathrm{~N}\left[(\mathrm{M}+\mathrm{H})^{+}\right] 200.1434$, found 200.1433; IR (film) $2956,2864,1602,1558,1507,1480,1448,1411,1377,1153,758 \mathrm{~cm}^{-1}$. Spectra data are consistent with those reported in the literature: Org. Lett. 2018, 20, 3487-3490.

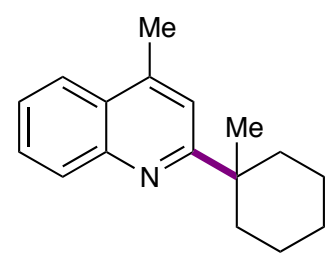

49

4-Methyl-2-(1-methylcyclohexyl)quinoline (49): According to the general procedure A, $\mathrm{FeSO}_{4} \cdot 7 \mathrm{H}_{2} \mathrm{O}$ (7.0 mg, $0.025 \mathrm{mmol}, 0.05$ equiv.), picolinic acid ( $6.2 \mathrm{mg}, 0.05 \mathrm{mmol}, 0.1$ equiv.), lepidine (72.3 mg, $0.50 \mathrm{mmol}, 1.0$ equiv.), 1-methylcyclohexanecarboxylic acid (718.2 mg, 5.0 mmol, 10 equiv.), $\mathrm{NaBrO}_{3}(150.9 \mathrm{mg}, 1.0 \mathrm{mmol}, 2.0$ equiv.), $4.5 \mathrm{~mL}$ of $\mathrm{H}_{2} \mathrm{O}$ and $0.5 \mathrm{~mL}$ of DMSO, $456 \mathrm{~nm}$ LEDs were used. After 24 hours, the reaction mixture was subjected to the workup procedure outlined in the general procedure and purified by flash chromatography ( $2 \%$ ethyl acetate/hexanes) to provide the title compound as a colorless oil (113.6 mg, 95\% yield). ${ }^{1} \mathrm{H} \mathrm{NMR}\left(400 \mathrm{MHz}, \mathrm{CDCl}_{3}\right) \delta 8.07$ (d, $J=8.3 \mathrm{~Hz}, 1 \mathrm{H}), 7.95(\mathrm{~d}, J=8.2 \mathrm{~Hz}, 1 \mathrm{H}), 7.66(\mathrm{t}, J=7.6 \mathrm{~Hz}, 1 \mathrm{H}), 7.50(\mathrm{t}, J=7.6 \mathrm{~Hz}$, 1H), $7.33(\mathrm{~s}, 1 \mathrm{H}), 2.69(\mathrm{~s}, 3 \mathrm{H}), 2.41-2.31(\mathrm{~m}, 2 \mathrm{H}), 1.67-1.55(\mathrm{~m}, 4 \mathrm{H}), 1.51-1.43(\mathrm{~m}$, 4H), $1.30(\mathrm{~s}, 3 \mathrm{H}) ;{ }^{13} \mathrm{C} \mathrm{NMR}\left(101 \mathrm{MHz}, \mathrm{CDCl}_{3}\right) \delta 168.16,147.52,143.48,129.90,128.55$, 126.42, 125.32, 123.36, 119.32, 41.24, 37.13, 29.13, 26.32, 22.90, 18.99; HRMS (ESI) $\mathrm{m} / \mathrm{z}$ calculated for $\mathrm{C}_{17} \mathrm{H}_{22} \mathrm{~N}\left[(\mathrm{M}+\mathrm{H})^{+}\right]$240.1747, found 240.1746; IR (film) 2925, 2855, $1602,1557,1506,1466,1446,1410,1338,950,758 \mathrm{~cm}^{-1}$. 


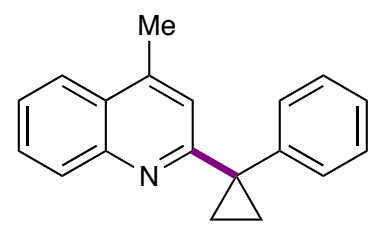

50

4-Methyl-2-(1-phenylcyclopropyl)quinoline (50): According to the general procedure $\mathrm{A}, \mathrm{FeSO}_{4} \cdot 7 \mathrm{H}_{2} \mathrm{O}(7.0 \mathrm{mg}, 0.025 \mathrm{mmol}, 0.05$ equiv.), picolinic acid (6.2 $\mathrm{mg}, 0.05 \mathrm{mmol}$, 0.1 equiv.), lepidine ( $72.3 \mathrm{mg}, 0.50 \mathrm{mmol}, 1.0$ equiv.), 1-phenylcyclopropanecarboxylic acid (413.8 mg, 2.5 mmol, 5 equiv.), $\mathrm{NaBrO}_{3}$ (150.9 mg, 1.0 mmol, 2.0 equiv.), $4.0 \mathrm{~mL}$ of $\mathrm{H}_{2} \mathrm{O}$ and $1.0 \mathrm{~mL}$ of DMSO, $456 \mathrm{~nm}$ LEDs were used. After 24 hours, the reaction mixture was subjected to the workup procedure outlined in the general procedure and purified by flash chromatography ( $2 \%$ ethyl acetate/hexanes) to provide the title compound as a white solid (87.5 mg, 68\% yield). ${ }^{1} \mathrm{H}$ NMR (400 $\left.\mathrm{MHz}, \mathrm{CDCl}_{3}\right) \delta 8.05$ (d, $J=8.4 \mathrm{~Hz}, 1 \mathrm{H}), 7.91(\mathrm{~d}, J=8.3 \mathrm{~Hz}, 1 \mathrm{H}), 7.67(\mathrm{t}, J=7.6 \mathrm{~Hz}, 1 \mathrm{H}), 7.49(\mathrm{t}, J=7.4 \mathrm{~Hz}, 1 \mathrm{H})$, $7.44(\mathrm{~d}, J=7.5 \mathrm{~Hz}, 2 \mathrm{H}), 7.38(\mathrm{t}, J=7.5 \mathrm{~Hz}, 2 \mathrm{H}), 7.30(\mathrm{t}, J=7.2 \mathrm{~Hz}, 1 \mathrm{H}), 6.97(\mathrm{~s}, 1 \mathrm{H})$, $2.55(\mathrm{~s}, 3 \mathrm{H}), 1.85(\mathrm{q}, J=3.8 \mathrm{~Hz}, 2 \mathrm{H}), 1.38(\mathrm{q}, J=3.8 \mathrm{~Hz}, 2 \mathrm{H}) ;{ }^{13} \mathrm{C}$ NMR $(101 \mathrm{MHz}$, $\left.\mathrm{CDCl}_{3}\right) \delta 163.68,147.48,143.72,143.46,130.07,129.51,128.90,128.47,126.59,126.50$ 125.28, 123.47, 121.90, 32.08, 18.62, 17.16; HRMS (ESI) m/z calculated for $\mathrm{C}_{19} \mathrm{H}_{18} \mathrm{~N}$ $\left[(\mathrm{M}+\mathrm{H})^{+}\right]$260.1434, found 260.1432; IR (film) 3059, 3007, 2921, 1596, 1555, 1503, $1445,1404,1341,949,759 \mathrm{~cm}^{-1}$. Spectra data are consistent with those reported in the literature: CN109134362, 2019, A. 


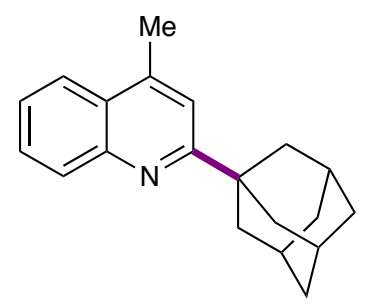

51

2-(Adamantan-1-yl)-4-methylquinoline (51): According to the general procedure B, $\mathrm{FeSO}_{4} \cdot 7 \mathrm{H}_{2} \mathrm{O}$ (14.0 mg, $0.05 \mathrm{mmol}, 0.1$ equiv.), picolinic acid (12.4 mg, $0.1 \mathrm{mmol}, 0.2$ equiv.), lepidine (72.3 mg, $0.50 \mathrm{mmol}, 1.0$ equiv.), adamantane-1-carboxylic acid (919.6 mg, 5.0 mmol, 10 equiv.), $\mathrm{NaClO}_{3}$ (161.3 mg, $1.5 \mathrm{mmol}, 3.0$ equiv.), $5.0 \mathrm{~mL}$ of DCE, $440 \mathrm{~nm}$ LEDs were used. After 24 hours, the reaction mixture was subjected to the workup procedure outlined in the general procedure and purified by flash chromatography ( $2 \%$ ethyl acetate/hexanes) to provide the title compound as a white solid (114.3 mg, 83\% yield). ${ }^{1} \mathrm{H}$ NMR (400 MHz, $\left.\mathrm{CDCl}_{3}\right) \delta 8.11(\mathrm{~d}, J=8.4 \mathrm{~Hz}, 1 \mathrm{H}), 7.95$ $(\mathrm{d}, J=8.2 \mathrm{~Hz}, 1 \mathrm{H}), 7.67(\mathrm{t}, J=7.6 \mathrm{~Hz}, 1 \mathrm{H}), 7.50(\mathrm{t}, J=7.6 \mathrm{~Hz}, 1 \mathrm{H}), 7.35(\mathrm{~s}, 1 \mathrm{H}), 2.70(\mathrm{~s}$, 3H), $2.18(\mathrm{~s}, 3 \mathrm{H}), 2.15(\mathrm{~s}, 6 \mathrm{H}), 1.86(\mathrm{~s}, 6 \mathrm{H}) ;{ }^{13} \mathrm{C} \mathrm{NMR}\left(101 \mathrm{MHz}, \mathrm{CDCl}_{3}\right) \delta 168.57$, $147.44,143.49,129.85,128.54,126.62,125.24,123.33,118.43,41.73,39.47,36.83$, 28.78, 18.89; HRMS (ESI) m/z calculated for $\mathrm{C}_{20} \mathrm{H}_{24} \mathrm{~N}\left[(\mathrm{M}+\mathrm{H})^{+}\right] 278.1903$, found 278.1902; IR (film) 2900, 2847, 1714, 1505, 1341, 1309, 1234, 1062, 975, 859, $755 \mathrm{~cm}^{-1}$. Spectra data are consistent with those reported in the literature: Org. Lett. 2018, 20, 32293232.<smiles>Cc1cc(C23CC4CC(CC(O)(C4)C2)C3)nc2ccccc12</smiles> 
3-(4-Methylquinolin-2-yl)adamantan-1-ol (52): According to the general procedure A, $\mathrm{FeSO}_{4} \cdot 7 \mathrm{H}_{2} \mathrm{O}$ (7.0 mg, $0.025 \mathrm{mmol}, 0.05$ equiv.), picolinic acid $(6.2 \mathrm{mg}, 0.05 \mathrm{mmol}, 0.1$ equiv.), lepidine (72.3 mg, $0.50 \mathrm{mmol}, 1.0$ equiv.), 3-hydroxyadamantane-1-carboxylic acid (1001.2 mg, 5.0 mmol, 10 equiv.), $\mathrm{NaBrO}_{3}(150.9 \mathrm{mg}, 1.0 \mathrm{mmol}, 2.0$ equiv.), 4.0 $\mathrm{mL}$ of $\mathrm{H}_{2} \mathrm{O}$ and $1.0 \mathrm{~mL}$ of DMSO, $440 \mathrm{~nm}$ LEDs were used. After 24 hours, the reaction mixture was subjected to the workup procedure outlined in the general procedure and purified by flash chromatography ( $20 \%$ ethyl acetate/hexanes) to provide the title compound as a white solid (109.1 mg, 74\% yield). ${ }^{1} \mathrm{H}$ NMR (400 MHz, $\left.\mathrm{CDCl}_{3}\right) \delta 8.06$ (d, $J=8.4 \mathrm{~Hz}, 1 \mathrm{H}), 7.93(\mathrm{~d}, J=8.3 \mathrm{~Hz}, 1 \mathrm{H}), 7.66(\mathrm{t}, J=7.6 \mathrm{~Hz}, 1 \mathrm{H}), 7.49$ (t, $J=7.6 \mathrm{~Hz}, 1 \mathrm{H})$, $7.29(\mathrm{~s}, 1 \mathrm{H}), 2.68(\mathrm{~s}, 3 \mathrm{H}), 2.38(\mathrm{~s}, 2 \mathrm{H}), 2.09(\mathrm{~s}, 2 \mathrm{H}), 2.07-1.93(\mathrm{~m}, 4 \mathrm{H}), 1.85-1.77(\mathrm{~m}$, 4H), $1.73(\mathrm{~s}, 1 \mathrm{H}), 1.72-1.60(\mathrm{~m}, 2 \mathrm{H}) ;{ }^{13} \mathrm{C} \mathrm{NMR}\left(101 \mathrm{MHz}, \mathrm{CDCl}_{3}\right) \delta$ 166.76, 147.33, $143.88,129.79,128.73,126.68,125.46,123.37,118.34,69.18,49.13,44.45,43.19,40.53$, 35.23, 30.86, 18.91; HRMS (ESI) m/z calculated for $\mathrm{C}_{20} \mathrm{H}_{24} \mathrm{NO}\left[(\mathrm{M}+\mathrm{H})^{+}\right]$294.1852, found 294.1851; IR (film) 3436, 2902, 2849, 1600, 1445, 1338, 1307, 1087, 1028, 944, $755 \mathrm{~cm}^{-1}$.

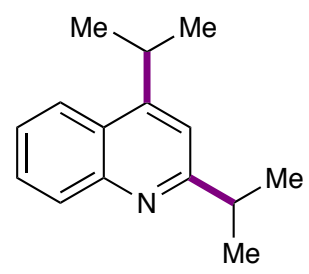

53

2,4-Diisopropylquinoline (53): According to the general procedure $\mathrm{A}, \mathrm{FeSO}_{4} \cdot 7 \mathrm{H}_{2} \mathrm{O}(7.0$ $\mathrm{mg}, 0.025 \mathrm{mmol}, 0.05$ equiv.), picolinic acid ( $6.2 \mathrm{mg}, 0.05 \mathrm{mmol}, 0.1$ equiv.), quinoline (65.0 mg, $0.50 \mathrm{mmol}, 1.0$ equiv.), isobutyric acid (445.0 mg, $5.0 \mathrm{mmol}, 10$ equiv.), $\mathrm{NaBrO}_{3}$ (150.9 mg, $1.0 \mathrm{mmol}, 2.0$ equiv.), $4.5 \mathrm{~mL}$ of $\mathrm{H}_{2} \mathrm{O}$ and $0.5 \mathrm{~mL}$ of DMSO, $456 \mathrm{~nm}$ LEDs were used. After 24 hours, the reaction mixture was subjected to the workup 
procedure outlined in the general procedure and purified by flash chromatography ( $10 \%$ ethyl acetate/hexanes) to provide the title compound as a colorless oil (68.8 mg, $64 \%$ yield). ${ }^{1} \mathrm{H}$ NMR (400 MHz, $\left.\mathrm{CDCl}_{3}\right) \delta 8.08(\mathrm{~d}, J=8.4 \mathrm{~Hz}, 1 \mathrm{H}), 8.04(\mathrm{~d}, J=8.4 \mathrm{~Hz}, 1 \mathrm{H})$, 7.65 (ddd, $J=8.4,6.8,1.4 \mathrm{~Hz}, 1 \mathrm{H}), 7.49$ (ddd, $J=8.4,6.8,1.3 \mathrm{~Hz}, 1 \mathrm{H}), 7.24$ (s, 1H), 3.72 (hept, $J=6.8 \mathrm{~Hz}, 1 \mathrm{H}), 3.25$ (hept, $J=6.9 \mathrm{~Hz}, 1 \mathrm{H}), 1.41(\mathrm{~d}, J=7.0 \mathrm{~Hz}, 12 \mathrm{H}) ;{ }^{13} \mathrm{C}$ NMR (101 MHz, $\left.\mathrm{CDCl}_{3}\right) \delta 167.40,154.37,147.87,129.80,128.57,125.55,125.24$, $122.81,114.68,37.41,28.34,22.92,22.55 ;$ HRMS (ESI) m/z calculated for $\mathrm{C}_{15} \mathrm{H}_{20} \mathrm{~N}$ $\left[(\mathrm{M}+\mathrm{H})^{+}\right]$214.1590, found 214.1589; IR (film) 2962, 1599, 1560, 1505, 1457, 1385, $1148,1090,941,879,792,759 \mathrm{~cm}^{-1}$. Spectra data are consistent with those reported in the literature: Chem. - Eur. J. 2015, 21, 17618-17622.

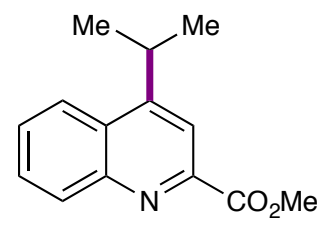

54

Methyl 4-isopropylquinoline-2-carboxylate (54): According to the general procedure A, $\mathrm{FeSO}_{4} \cdot 7 \mathrm{H}_{2} \mathrm{O}$ (7.0 mg, $0.025 \mathrm{mmol}, 0.05$ equiv.), picolinic acid $(6.2 \mathrm{mg}, 0.05 \mathrm{mmol}, 0.1$ equiv.), methyl quinoline-2-carboxylate ( $95.0 \mathrm{mg}, 0.50 \mathrm{mmol}, 1.0$ equiv.), isobutyric acid (445.0 mg, $5.0 \mathrm{mmol}, 10$ equiv.), $\mathrm{NaBrO}_{3}(150.9 \mathrm{mg}, 1.0 \mathrm{mmol}, 2.0$ equiv.), $4.5 \mathrm{~mL}$ of $\mathrm{H}_{2} \mathrm{O}$ and $0.5 \mathrm{~mL}$ of DMSO, $456 \mathrm{~nm}$ LEDs were used. After 24 hours, the reaction mixture was subjected to the workup procedure outlined in the general procedure and purified by flash chromatography ( $10 \%$ ethyl acetate/hexanes) to provide the title compound as a light yellow oil (57.3 mg, $50 \%$ yield). ${ }^{1} \mathrm{H}$ NMR $\left(400 \mathrm{MHz}, \mathrm{CDCl}_{3}\right) \delta 8.29$ (d, $J=8.5 \mathrm{~Hz}, 1 \mathrm{H}), 8.11(\mathrm{~d}, J=8.5 \mathrm{~Hz}, 1 \mathrm{H}), 8.10(\mathrm{~s}, 1 \mathrm{H}), 7.72(\mathrm{ddd}, J=8.4,6.8,1.4 \mathrm{~Hz}$, 1H), 7.62 (ddd, $J=8.4,6.8,1.4 \mathrm{~Hz}, 1 \mathrm{H}), 4.05$ (s, 3H), 3.75 (hept, $J=6.8 \mathrm{~Hz}, 1 \mathrm{H}), 1.40$ 
$(\mathrm{d}, J=6.9 \mathrm{~Hz}, 6 \mathrm{H}) ;{ }^{13} \mathrm{C} \mathrm{NMR}\left(101 \mathrm{MHz}, \mathrm{CDCl}_{3}\right) \delta 166.22,156.04,147.65,147.61$, 131.50, 129.54, 128.18, 127.88, 122.89, 116.80, 53.05, 28.53, 22.76; HRMS (ESI) m/z calculated for $\mathrm{C}_{14} \mathrm{H}_{16} \mathrm{NO}_{2}\left[(\mathrm{M}+\mathrm{H})^{+}\right]$230.1176, found 230.1176; IR (film) 2964, 1717, $1436,1259,1242,1147,1113,1080,911,761 \mathrm{~cm}^{-1}$. Spectra data are consistent with those reported in the literature: J. Am. Chem. Soc. 2017, 139. 14315-14321.

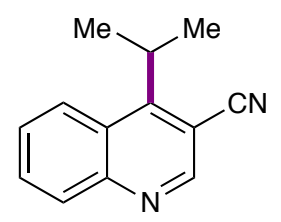

55

4-Isopropylquinoline-3-carbonitrile (55): According to the general procedure A, $\mathrm{FeSO}_{4} \cdot 7 \mathrm{H}_{2} \mathrm{O}$ (7.0 mg, $0.025 \mathrm{mmol}, 0.05$ equiv.), picolinic acid (6.2 mg, $0.05 \mathrm{mmol}, 0.1$ equiv.), quinoline-3-carbonitrile ( $81.0 \mathrm{mg}, 0.50 \mathrm{mmol}, 1.0$ equiv.), isobutyric acid (445.0 mg, 5.0 mmol, 10 equiv.), $\mathrm{NaBrO}_{3}\left(150.9 \mathrm{mg}, 1.0 \mathrm{mmol}, 2.0\right.$ equiv.), $4.0 \mathrm{~mL}$ of $\mathrm{H}_{2} \mathrm{O}$ and $1.0 \mathrm{~mL}$ of DMSO, $440 \mathrm{~nm}$ LEDs were used. After 24 hours, the reaction mixture was subjected to the workup procedure outlined in the general procedure and purified by flash chromatography (10\% ethyl acetate/hexanes) to provide the title compound as a white solid (80.0 mg, 81\% yield). ${ }^{1} \mathrm{H}$ NMR (400 MHz, $\left.\mathrm{CDCl}_{3}\right) \delta 8.92(\mathrm{~s}, 1 \mathrm{H}), 8.28(\mathrm{~d}, J=8.5$ $\mathrm{Hz}, 1 \mathrm{H}), 8.14(\mathrm{~d}, J=9.1 \mathrm{~Hz}, 1 \mathrm{H}), 7.82(\mathrm{ddd}, J=8.4,6.9,1.4 \mathrm{~Hz}, 1 \mathrm{H}), 7.65(\mathrm{ddd}, J=8.5$, 6.8, 1.4 Hz, 1H), 4.02 (hept, $J=7.1 \mathrm{~Hz}, 1 \mathrm{H}), 1.62$ (d, $J=7.2 \mathrm{~Hz}, 6 \mathrm{H}) ;{ }^{13} \mathrm{C}$ NMR $(101$ $\left.\mathrm{MHz}, \mathrm{CDCl}_{3}\right) \delta 160.19,151.14,148.98,131.83,130.80,127.70,125.18,124.12,117.64$, 104.73, 30.49, 21.45; HRMS (ESI) m/z calculated for $\mathrm{C}_{13} \mathrm{H}_{13} \mathrm{~N}_{2}\left[(\mathrm{M}+\mathrm{H})^{+}\right]$197.1073, found 197.1073; IR (film) 2966, 2222, 1577, 1500, 1460, 1387, 1197, 1104, 1027, 917, $761 \mathrm{~cm}^{-1}$. Spectra data are consistent with those reported in the literature: J. Am. Chem. Soc. 2017, 139, 14315-14321. 


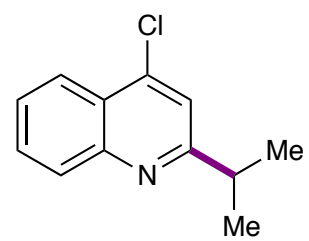

56

4-Chloro-2-isopropylquinoline (56): According to the general procedure A,

$\mathrm{FeSO}_{4} \cdot 7 \mathrm{H}_{2} \mathrm{O}(7.0 \mathrm{mg}, 0.025 \mathrm{mmol}, 0.05$ equiv.), picolinic acid (6.2 mg, $0.05 \mathrm{mmol}, 0.1$ equiv.), 4-chloroquinoline (82.0 mg, $0.50 \mathrm{mmol}, 1.0$ equiv.), isobutyric acid (445.0 mg, 5.0 mmol, 10 equiv.), $\mathrm{NaBrO}_{3}$ (150.9 mg, $1.0 \mathrm{mmol}, 2.0$ equiv.), $4.5 \mathrm{~mL}$ of $\mathrm{H}_{2} \mathrm{O}$ and 0.5 $\mathrm{mL}$ of DMSO, $456 \mathrm{~nm}$ LEDs were used. After 24 hours, the reaction mixture was subjected to the workup procedure outlined in the general procedure and purified by flash chromatography (5\% ethyl acetate/hexanes) to provide the title compound as a light yellow oil (69.9 mg, 68\% yield). ${ }^{1} \mathrm{H}$ NMR $\left(400 \mathrm{MHz}, \mathrm{CDCl}_{3}\right) \delta 8.17(\mathrm{~d}, J=8.3 \mathrm{~Hz}, 1 \mathrm{H})$, $8.06(\mathrm{~d}, J=8.4 \mathrm{~Hz}, 1 \mathrm{H}), 7.72(\mathrm{ddd}, J=8.5,6.9,1.5 \mathrm{~Hz}, 1 \mathrm{H}), 7.56(\mathrm{ddd}, J=8.2,6.9,1.2$ $\mathrm{Hz}, 1 \mathrm{H}), 7.42(\mathrm{~s}, 1 \mathrm{H}), 3.23$ (hept, $J=6.9 \mathrm{~Hz}, 1 \mathrm{H}), 1.39(\mathrm{~d}, J=6.9 \mathrm{~Hz}, 6 \mathrm{H}) ;{ }^{13} \mathrm{C} \mathrm{NMR}$ $\left(101 \mathrm{MHz}, \mathrm{CDCl}_{3}\right) \delta 167.57,148.50,142.67,130.14,129.24,126.59,125.05,123.83$, 119.32, 37.12, 22.32; HRMS (ESI) m/z calculated for $\mathrm{C}_{12} \mathrm{H}_{13} \mathrm{ClN}\left[(\mathrm{M}+\mathrm{H})^{+}\right]$206.0731, found 206.0730; IR (film) 2963, 1589, 1552, 1493, 1148, 1088, 979, 919, 837, $757 \mathrm{~cm}^{-1}$. Spectra data are consistent with those reported in the literature: Org. Lett. 2017, 19, 65946597.<smiles>Cc1cc(C(C)C)c2ccc(Cl)cc2n1</smiles>

57 
7-Chloro-4-isopropyl-2-methylquinoline (57): According to the general procedure A, $\mathrm{FeSO}_{4} \cdot 7 \mathrm{H}_{2} \mathrm{O}(7.0 \mathrm{mg}, 0.025 \mathrm{mmol}, 0.05$ equiv.), picolinic acid (6.2 mg, $0.05 \mathrm{mmol}, 0.1$ equiv.), 7-chloro-2-methylquinoline (90.0 mg, $0.50 \mathrm{mmol}, 1.0$ equiv.), isobutyric acid (445.0 mg, 5.0 mmol, 10 equiv.), $\mathrm{NaBrO}_{3}$ (150.9 mg, $1.0 \mathrm{mmol}, 2.0$ equiv.), $4.5 \mathrm{~mL}$ of $\mathrm{H}_{2} \mathrm{O}$ and $0.5 \mathrm{~mL}$ of DMSO, $456 \mathrm{~nm}$ LEDs were used. After 24 hours, the reaction mixture was subjected to the workup procedure outlined in the general procedure and purified by flash chromatography ( $5 \%$ ethyl acetate/hexanes) to provide the title compound as a colorless oil (106.7 mg, 97\% yield). ${ }^{1} \mathrm{H} \mathrm{NMR}\left(400 \mathrm{MHz}, \mathrm{CDCl}_{3}\right) \delta 8.02$ $(\mathrm{d}, J=2.1 \mathrm{~Hz}, 1 \mathrm{H}), 7.94(\mathrm{~d}, J=9.0 \mathrm{~Hz}, 1 \mathrm{H}), 7.41(\mathrm{dd}, J=9.0,2.2 \mathrm{~Hz}, 1 \mathrm{H}), 7.16(\mathrm{~s}, 1 \mathrm{H})$, 3.62 (hept, $J=6.8 \mathrm{~Hz}, 1 \mathrm{H}), 2.70(\mathrm{~s}, 3 \mathrm{H}), 1.36(\mathrm{~d}, J=6.9 \mathrm{~Hz}, 6 \mathrm{H}) ;{ }^{13} \mathrm{C}$ NMR $(101 \mathrm{MHz}$, $\left.\mathrm{CDCl}_{3}\right) \delta 160.05,154.47,148.42,134.64,128.25,126.21,124.29,123.55,117.95,28.30$, 25.35, 22.80; HRMS (ESI) m/z calculated for $\mathrm{C}_{13} \mathrm{H}_{15} \mathrm{ClN}\left[(\mathrm{M}+\mathrm{H})^{+}\right] 220.0888$, found 220.0887; IR (film) 2965, 1600, 1496, 1332, 1187, 1074, 955, 880, 821, $770 \mathrm{~cm}^{-1}$.

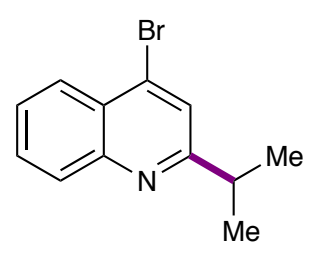

58

4-Bromo-2-isopropylquinoline (58): According to the general procedure A, $\mathrm{FeSO}_{4} \cdot 7 \mathrm{H}_{2} \mathrm{O}(7.0 \mathrm{mg}, 0.025 \mathrm{mmol}, 0.05$ equiv.), picolinic acid (6.2 mg, $0.05 \mathrm{mmol}, 0.1$ equiv.), 4-bromoquinoline (105.0 mg, $0.50 \mathrm{mmol}, 1.0$ equiv.), isobutyric acid (445.0 mg, 5.0 mmol, 10 equiv.), $\mathrm{NaBrO}_{3}$ (150.9 mg, $1.0 \mathrm{mmol}, 2.0$ equiv.), $4.0 \mathrm{~mL}$ of $\mathrm{H}_{2} \mathrm{O}$ and 1.0 $\mathrm{mL}$ of DMSO, $440 \mathrm{~nm}$ LEDs were used. After 24 hours, the reaction mixture was subjected to the workup procedure outlined in the general procedure and purified by flash chromatography ( $5 \%$ ethyl acetate/hexanes) to provide the title compound as a light 
yellow oil (84.2 mg, 67\% yield). ${ }^{1} \mathrm{H}$ NMR (400 MHz, $\left.\mathrm{CDCl}_{3}\right) \delta 8.13(\mathrm{~d}, J=8.4 \mathrm{~Hz}, 1 \mathrm{H})$, $8.04(\mathrm{~d}, J=8.4 \mathrm{~Hz}, 1 \mathrm{H}), 7.72(\mathrm{ddd}, J=8.4,6.9,1.4 \mathrm{~Hz}, 1 \mathrm{H}), 7.63(\mathrm{~s}, 1 \mathrm{H}), 7.56$ (ddd, $J=$ 8.2, 6.9, 1.2 Hz, 1H), 3.23 (hept, $J=6.9 \mathrm{~Hz}, 1 \mathrm{H}), 1.39$ (d, $J=6.9 \mathrm{~Hz}, 6 \mathrm{H}) ;{ }^{13} \mathrm{C} \mathrm{NMR}$ $\left(101 \mathrm{MHz}, \mathrm{CDCl}_{3}\right) \delta 167.52,148.29,134.31,130.19,129.31,126.89,126.50,126.42$, 123.25, 36.98, 22.34; HRMS (ESI) m/z calculated for $\mathrm{C}_{12} \mathrm{H}_{13} \mathrm{BrN}\left[(\mathrm{M}+\mathrm{H})^{+}\right] 250.0226$, found 250.0226; IR (film) 2962, 1614, 1550, 1456, 1295, 1147, 1087, 911, 866, 815, 756 $\mathrm{cm}^{-1}$.<smiles>Cc1cc(C(C)C)c2cc(Br)ccc2n1</smiles>

59

6-Bromo-4-isopropyl-2-methylquinoline (59): According to the general procedure A, $\mathrm{FeSO}_{4} \cdot 7 \mathrm{H}_{2} \mathrm{O}$ (7.0 mg, $0.025 \mathrm{mmol}, 0.05$ equiv.), picolinic acid $(6.2 \mathrm{mg}, 0.05 \mathrm{mmol}, 0.1$ equiv.), 6-bromo-2-methylquinoline ( $113.0 \mathrm{mg}, 0.50 \mathrm{mmol}, 1.0$ equiv.), isobutyric acid (445.0 mg, $5.0 \mathrm{mmol}, 10$ equiv.), $\mathrm{NaBrO}_{3}(150.9 \mathrm{mg}, 1.0 \mathrm{mmol}, 2.0$ equiv.), $4.5 \mathrm{~mL}$ of $\mathrm{H}_{2} \mathrm{O}$ and $0.5 \mathrm{~mL}$ of DMSO, $456 \mathrm{~nm}$ LEDs were used. After 24 hours, the reaction mixture was subjected to the workup procedure outlined in the general procedure and purified by flash chromatography ( $10 \%$ ethyl acetate/hexanes) to provide the title compound as a yellow oil (118.1 mg, 89\% yield). ${ }^{1} \mathrm{H}$ NMR (400 MHz, $\left.\mathrm{CDCl}_{3}\right) \delta 8.17$ (d, $J=2.0 \mathrm{~Hz}, 1 \mathrm{H}), 7.91(\mathrm{~d}, J=8.9 \mathrm{~Hz}, 1 \mathrm{H}), 7.72(\mathrm{dd}, J=8.9,2.1 \mathrm{~Hz}, 1 \mathrm{H}), 7.20(\mathrm{~s}, 1 \mathrm{H})$, 3.60 (hept, $J=6.8 \mathrm{~Hz}, 1 \mathrm{H}), 2.71$ (s, 3H), $1.38(\mathrm{~d}, J=6.9 \mathrm{~Hz}, 6 \mathrm{H}) ;{ }^{13} \mathrm{C} \mathrm{NMR}(101 \mathrm{MHz}$, $\left.\mathrm{CDCl}_{3}\right) \delta 159.21,153.61,146.40,132.17,131.01,126.39,125.33,119.42,118.50,28.18$, 25.32, 22.79; HRMS (ESI) $\mathrm{m} / \mathrm{z}$ calculated for $\mathrm{C}_{13} \mathrm{H}_{15} \mathrm{BrN}\left[(\mathrm{M}+\mathrm{H})^{+}\right]$264.0382, found 264.0382; IR (film) 2964, 1598, 1488, 1384, 1333, 1218, 1070, 992, 950, 876, $826 \mathrm{~cm}^{-1}$. 


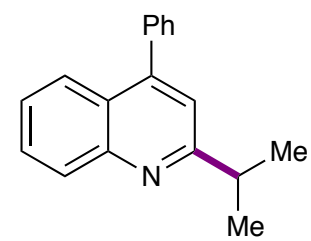

60

2-Isopropyl-4-phenylquinoline (60): According to the general procedure A, $\mathrm{FeSO}_{4} \cdot 7 \mathrm{H}_{2} \mathrm{O}$ (7.0 mg, $0.025 \mathrm{mmol}, 0.05$ equiv.), picolinic acid (6.2 mg, $0.05 \mathrm{mmol}, 0.1$ equiv.), 4-phenylquinoline (104.5 mg, $0.50 \mathrm{mmol}, 1.0$ equiv.), isobutyric acid (445.0 mg, 5.0 mmol, 10 equiv.), $\mathrm{NaBrO}_{3}$ (150.9 mg, $1.0 \mathrm{mmol}, 2.0$ equiv.), $4.5 \mathrm{~mL}$ of $\mathrm{H}_{2} \mathrm{O}$ and 0.5 $\mathrm{mL}$ of DMSO, $456 \mathrm{~nm}$ LEDs were used. After 24 hours, the reaction mixture was subjected to the workup procedure outlined in the general procedure and purified by flash chromatography (10\% ethyl acetate/hexanes) to provide the title compound as a colorless oil (101.7 mg, 82\% yield). ${ }^{1} \mathrm{H}$ NMR $\left(400 \mathrm{MHz}, \mathrm{CDCl}_{3}\right) \delta 8.16(\mathrm{~d}, J=8.4 \mathrm{~Hz}, 1 \mathrm{H}), 7.88$ $(\mathrm{d}, J=8.4 \mathrm{~Hz}, 1 \mathrm{H}), 7.69(\mathrm{ddd}, J=8.4,6.8,1.5 \mathrm{~Hz}, 1 \mathrm{H}), 7.57-7.46(\mathrm{~m}, 5 \mathrm{H}), 7.43$ (ddd, $J$ $=8.3,6.8,1.3 \mathrm{~Hz}, 1 \mathrm{H}), 7.30(\mathrm{~s}, 1 \mathrm{H}), 3.33$ (hept, $J=6.9 \mathrm{~Hz}, 1 \mathrm{H}), 1.45(\mathrm{~d}, J=7.0 \mathrm{~Hz}, 6 \mathrm{H})$; ${ }^{13} \mathrm{C}$ NMR $\left(101 \mathrm{MHz}, \mathrm{CDCl}_{3}\right) \delta 167.09,148.74,148.08,138.43,129.48,129.25,129.11$, 128.44, 128.20, 125.67, 125.53, 125.43, 119.31, 37.25, 22.53; HRMS (ESI) m/z calculated for $\mathrm{C}_{18} \mathrm{H}_{18} \mathrm{~N}\left[(\mathrm{M}+\mathrm{H})^{+}\right]$248.1434, found 248.1433; IR (film) 2971, 1562, 1464 , $1361,1314,1134,1109,1085,913,847,758 \mathrm{~cm}^{-1}$. Spectra data are consistent with those reported in the literature: J. Am. Chem. Soc. 2017, 139, 14315-14321.<smiles>COc1cc(C(C)C)nc2ccccc12</smiles>

61 
2-Isopropyl-4-methoxyquinoline (61): According to the general procedure A, $\mathrm{FeSO}_{4} \cdot 7 \mathrm{H}_{2} \mathrm{O}$ (7.0 mg, $0.025 \mathrm{mmol}, 0.05$ equiv.), picolinic acid (6.2 mg, $0.05 \mathrm{mmol}, 0.1$ equiv.), 4-methoxyquinoline (83.5 $\mathrm{mg}, 0.50 \mathrm{mmol}, 1.0$ equiv.), isobutyric acid (445.0 $\mathrm{mg}$, 5.0 mmol, 10 equiv.), $\mathrm{NaBrO}_{3}$ (150.9 mg, $1.0 \mathrm{mmol}, 2.0$ equiv.), $4.0 \mathrm{~mL}$ of $\mathrm{H}_{2} \mathrm{O}$ and 1.0 $\mathrm{mL}$ of DMSO, $440 \mathrm{~nm}$ LEDs were used. After 24 hours, the reaction mixture was subjected to the workup procedure outlined in the general procedure and purified by flash chromatography ( $5 \%$ ethyl acetate/hexanes) to provide the title compound as a light yellow oil (73.2 mg, 72\% yield). ${ }^{1} \mathrm{H}$ NMR $\left(400 \mathrm{MHz}, \mathrm{CDCl}_{3}\right) \delta 8.13(\mathrm{~d}, J=8.1 \mathrm{~Hz}, 1 \mathrm{H})$, $7.99(\mathrm{~d}, J=8.4 \mathrm{~Hz}, 1 \mathrm{H}), 7.64(\mathrm{t}, J=7.7 \mathrm{~Hz}, 1 \mathrm{H}), 7.42(\mathrm{t}, J=7.3 \mathrm{~Hz}, 1 \mathrm{H}), 6.65(\mathrm{~s}, 1 \mathrm{H})$, $4.03(\mathrm{~s}, 3 \mathrm{H}), 3.22$ (hept, $J=6.9 \mathrm{~Hz}, 1 \mathrm{H}), 1.39(\mathrm{~d}, J=7.0 \mathrm{~Hz}, 6 \mathrm{H}) ;{ }^{13} \mathrm{C}$ NMR $(101 \mathrm{MHz}$, $\left.\mathrm{CDCl}_{3}\right) \delta 168.89,162.52,148.40,129.55,128.20,124.71,121.48,120.15,97.30,55.37$, 37.68, 22.51; HRMS (ESI) m/z calculated for $\mathrm{C}_{13} \mathrm{H}_{16} \mathrm{NO}\left[(\mathrm{M}+\mathrm{H})^{+}\right] 202.1226$, found 202.1226; IR (film) 2961, 1594, 1564, 1505, 1350, 1196, 1111, 991, 947, 837, $763 \mathrm{~cm}^{-1}$.<smiles>CC(C)c1cc(O)nc2ccccc12</smiles>

62

4-Isopropylquinolin-2-ol (62): According to the general procedure $\mathrm{B}, \mathrm{FeSO}_{4} \cdot 7 \mathrm{H}_{2} \mathrm{O}(7.0$ mg, $0.025 \mathrm{mmol}, 0.05$ equiv.), picolinic acid (6.2 mg, $0.05 \mathrm{mmol}, 0.1$ equiv.), quinoline2-ol (72.5 mg, $0.50 \mathrm{mmol}, 1.0$ equiv.), isobutyric acid (445.0 mg, $5.0 \mathrm{mmol}, 10$ equiv.), $\mathrm{NaClO}_{3}$ (106.5 mg, $1.0 \mathrm{mmol}, 2.0$ equiv.), $4.0 \mathrm{~mL}$ of $\mathrm{H}_{2} \mathrm{O}$ and $1.0 \mathrm{~mL}$ of DMSO, $427 \mathrm{~nm}$ LEDs were used. After 36 hours, the reaction mixture was subjected to the workup procedure outlined in the general procedure and purified by flash chromatography $(33 \%$ ethyl acetate/hexanes) to provide the title compound as a white solid ( $83.5 \mathrm{mg}, 89 \%$ 
yield). ${ }^{1} \mathrm{H}$ NMR (400 MHz, $\left.\mathrm{CDCl}_{3}\right) \delta 12.13(\mathrm{~s}, 1 \mathrm{H}), 7.62(\mathrm{~s}, 1 \mathrm{H}), 7.54(\mathrm{~d}, J=7.7 \mathrm{~Hz}, 1 \mathrm{H})$, 7.46 (ddd, $J=8.3,6.9,1.4 \mathrm{~Hz}, 1 \mathrm{H}), 7.40(\mathrm{~d}, J=7.8 \mathrm{~Hz}, 1 \mathrm{H}), 7.19$ (ddd, $J=8.0,7.0,1.4$ $\mathrm{Hz}, 1 \mathrm{H}), 3.37$ (hept, $J=6.8 \mathrm{~Hz}, 1 \mathrm{H}), 1.32(\mathrm{~d}, J=6.9 \mathrm{~Hz}, 6 \mathrm{H}) ;{ }^{13} \mathrm{C} \mathrm{NMR}(101 \mathrm{MHz}$, $\left.\mathrm{CDCl}_{3}\right) \delta 163.97,139.81,137.08,133.97,129.32,127.17,122.35,120.34,115.54,27.51$, 21.86; HRMS (ESI) $\mathrm{m} / \mathrm{z}$ calculated for $\mathrm{C}_{12} \mathrm{H}_{14} \mathrm{NO}\left[(\mathrm{M}+\mathrm{H})^{+}\right]$188.1070, found 188.1069; IR (film) 2966, 1652, 1518, 1462, 1335, 1254, 1197, 1103, 983, 768, $733 \mathrm{~cm}^{-1}$.<smiles>CC(=O)c1cc2ccccc2c(C(C)C)n1</smiles>

63

Methyl 1-isopropylisoquinoline-3-carboxylate (63): According to the general procedure $\mathrm{A}, \mathrm{FeSO}_{4} \cdot 7 \mathrm{H}_{2} \mathrm{O}(7.0 \mathrm{mg}, 0.025 \mathrm{mmol}, 0.05$ equiv.), picolinic acid $(6.2 \mathrm{mg}$, $0.05 \mathrm{mmol}, 0.1$ equiv.), methyl isoquinoline-3-carboxylate ( $93.5 \mathrm{mg}, 0.50 \mathrm{mmol}, 1.0$ equiv.), isobutyric acid (445.0 mg, $5.0 \mathrm{mmol}, 10$ equiv.), $\mathrm{NaBrO}_{3}(150.9 \mathrm{mg}, 1.0 \mathrm{mmol}$, 2.0 equiv.), $4.5 \mathrm{~mL}$ of $\mathrm{H}_{2} \mathrm{O}$ and $0.5 \mathrm{~mL}$ of DMSO, $456 \mathrm{~nm}$ LEDs were used. After 24 hours, the reaction mixture was subjected to the workup procedure outlined in the general procedure and purified by flash chromatography (5\% ethyl acetate/hexanes) to provide the title compound as a white solid (70.5 $\mathrm{mg}, 62 \%$ yield). ${ }^{1} \mathrm{H}$ NMR $\left(400 \mathrm{MHz}, \mathrm{CDCl}_{3}\right) \delta$ $8.40(\mathrm{~s}, 1 \mathrm{H}), 8.30-8.22(\mathrm{~m}, 1 \mathrm{H}), 7.96-7.89(\mathrm{~m}, 1 \mathrm{H}), 7.74-7.64(\mathrm{~m}, 2 \mathrm{H}), 4.02(\mathrm{~s}, 3 \mathrm{H})$, 3.95 (hept, $J=6.8 \mathrm{~Hz}, 1 \mathrm{H}), 1.49(\mathrm{~d}, J=6.8 \mathrm{~Hz}, 6 \mathrm{H}) ;{ }^{13} \mathrm{C} \mathrm{NMR}\left(101 \mathrm{MHz}, \mathrm{CDCl}_{3}\right) \delta$ $166.75,166.61,140.51,135.95,130.07,129.03,128.97,127.62,124.90,122.48,52.57$, 31.64, 21.95; HRMS (ESI) $\mathrm{m} / \mathrm{z}$ calculated for $\mathrm{C}_{14} \mathrm{H}_{16} \mathrm{NO}_{2}\left[(\mathrm{M}+\mathrm{H})^{+}\right] 230.1176$, found 230.1175; IR (film) 2968, 1733, 1357, 1322, 1146, 1116, 1102, 1077, 979, 897, 778, 754, 
$677 \mathrm{~cm}^{-1}$. Spectra data are consistent with those reported in the literature: J. Am. Chem.

Soc. 2008, 130, 1558-1559.<smiles>CC(=O)c1cnc(C(C)C)c2ccccc12</smiles>

Methyl 1-isopropylisoquinoline-4-carboxylate (64): According to the general procedure $\mathrm{A}, \mathrm{FeSO}_{4} \cdot 7 \mathrm{H}_{2} \mathrm{O}(7.0 \mathrm{mg}, 0.025 \mathrm{mmol}, 0.05$ equiv.), picolinic acid $(6.2 \mathrm{mg}$, $0.05 \mathrm{mmol}, 0.1$ equiv.), methyl isoquinoline-4-carboxylate ( $96.0 \mathrm{mg}, 0.50 \mathrm{mmol}, 1.0$ equiv.), isobutyric acid (445.0 mg, $5.0 \mathrm{mmol}, 10$ equiv.), $\mathrm{NaBrO}_{3}(150.9 \mathrm{mg}, 1.0 \mathrm{mmol}$, 2.0 equiv.), $4.0 \mathrm{~mL}$ of $\mathrm{H}_{2} \mathrm{O}$ and $1.0 \mathrm{~mL}$ of DMSO, $440 \mathrm{~nm}$ LEDs were used. After 24 hours, the reaction mixture was subjected to the workup procedure outlined in the general procedure and purified by flash chromatography ( $10 \%$ ethyl acetate/hexanes) to provide the title compound as a light yellow oil $\left(74.0 \mathrm{mg}, 64 \%\right.$ yield). ${ }^{1} \mathrm{H}$ NMR (400 MHz, $\left.\mathrm{CDCl}_{3}\right) \delta 9.13(\mathrm{~s}, 1 \mathrm{H}), 8.97(\mathrm{~d}, J=8.6 \mathrm{~Hz}, 1 \mathrm{H}), 8.27(\mathrm{~d}, J=8.5 \mathrm{~Hz}, 1 \mathrm{H}), 7.77$ (ddd, $J=$ 8.4, 6.8, $1.3 \mathrm{~Hz}, 1 \mathrm{H}), 7.62$ (ddd, $J=8.3,6.8,1.3 \mathrm{~Hz}, 1 \mathrm{H}), 4.05-3.93(\mathrm{~m}, 4 \mathrm{H}), 1.44$ (d, $J$ $=6.8 \mathrm{~Hz}, 6 \mathrm{H}) ;{ }^{13} \mathrm{C} \mathrm{NMR}\left(101 \mathrm{MHz}, \mathrm{CDCl}_{3}\right) \delta 171.28,167.17,145.76,134.22,131.18$, 127.14, 125.81, 125.70, 124.96, 118.68, 52.12, 31.45, 22.08; HRMS (ESI) m/z calculated for $\mathrm{C}_{14} \mathrm{H}_{16} \mathrm{NO}_{2}\left[(\mathrm{M}+\mathrm{H})^{+}\right]$230.1176, found 230.1175; IR (film) 2965, 1714, 1556, 1507, $1434,1298,1220,1130,1032,926,782 \mathrm{~cm}^{-1}$.<smiles>CC(C)c1ncc(C#N)c2ccccc12</smiles>

65 
1-Isopropylisoquinoline-4-carbonitrile (65): According to the general procedure A, $\mathrm{FeSO}_{4} \cdot 7 \mathrm{H}_{2} \mathrm{O}$ (7.0 mg, $0.025 \mathrm{mmol}, 0.05$ equiv.), picolinic acid (6.2 mg, $0.05 \mathrm{mmol}, 0.1$ equiv.), isoquinoline-4-carbonitrile (79.0 $\mathrm{mg}, 0.50 \mathrm{mmol}, 1.0$ equiv.), isobutyric acid (445.0 mg, 5.0 mmol, 10 equiv.), $\mathrm{NaBrO}_{3}(150.9 \mathrm{mg}, 1.0 \mathrm{mmol}, 2.0$ equiv.), $4.0 \mathrm{~mL}$ of $\mathrm{H}_{2} \mathrm{O}$ and $1.0 \mathrm{~mL}$ of DMSO, $440 \mathrm{~nm}$ LEDs were used. After 24 hours, the reaction mixture was subjected to the workup procedure outlined in the general procedure and purified by flash chromatography (10\% ethyl acetate/hexanes) to provide the title compound as a white solid (66.1 mg, 67\% yield). ${ }^{1} \mathrm{H}$ NMR (400 MHz, $\left.\mathrm{CDCl}_{3}\right) \delta 8.84(\mathrm{~s}$, 1H), $8.32(\mathrm{~d}, J=8.5 \mathrm{~Hz}, 1 \mathrm{H}), 8.18(\mathrm{~d}, J=8.3 \mathrm{~Hz}, 1 \mathrm{H}), 7.87$ (ddd, $J=8.2,7.0,1.2 \mathrm{~Hz}$, 1H), 7.74 (ddd, $J=8.3,6.9,1.3 \mathrm{~Hz}, 1 \mathrm{H}), 3.99$ (hept, $J=6.7 \mathrm{~Hz}, 1 \mathrm{H}), 1.44$ (d, $J=6.8 \mathrm{~Hz}$, $6 \mathrm{H}) ;{ }^{13} \mathrm{C} \mathrm{NMR}\left(101 \mathrm{MHz}, \mathrm{CDCl}_{3}\right) \delta 171.57,147.47,134.76,132.08,128.71,125.47$, 125.27, 124.99, 116.50, 104.00, 31.68, 21.99; HRMS (ESI) m/z calculated for $\mathrm{C}_{13} \mathrm{H}_{13} \mathrm{~N}_{2}$ $\left[(\mathrm{M}+\mathrm{H})^{+}\right]$197.1073, found 197.1073; IR (film) 2963, 2222, 1552, 1504, 1390, 1344, $1249,1066,998,917,761 \mathrm{~cm}^{-1}$.

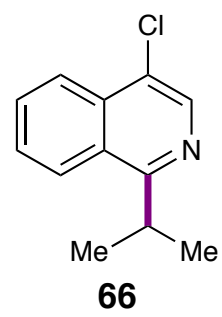

4-Chloro-1-isopropylisoquinoline (66): According to the general procedure A, $\mathrm{FeSO}_{4} \cdot 7 \mathrm{H}_{2} \mathrm{O}(7.0 \mathrm{mg}, 0.025 \mathrm{mmol}, 0.05$ equiv.), picolinic acid (6.2 mg, $0.05 \mathrm{mmol}, 0.1$ equiv.), 4-chloroisoquinoline ( $83.5 \mathrm{mg}, 0.50 \mathrm{mmol}, 1.0$ equiv.), isobutyric acid (445.0 mg, 5.0 mmol, 10 equiv.), $\mathrm{NaBrO}_{3}$ (150.9 mg, $1.0 \mathrm{mmol}, 2.0$ equiv.), $4.5 \mathrm{~mL}$ of $\mathrm{H}_{2} \mathrm{O}$ and 0.5 $\mathrm{mL}$ of DMSO, $456 \mathrm{~nm}$ LEDs were used. After 24 hours, the reaction mixture was subjected to the workup procedure outlined in the general procedure and purified by flash 
chromatography (5\% ethyl acetate/hexanes) to provide the title compound as a light yellow solid (63.5 mg, 64\% yield). ${ }^{1} \mathrm{H}$ NMR (400 MHz, $\left.\mathrm{CDCl}_{3}\right) \delta 8.53(\mathrm{~s}, 1 \mathrm{H}), 8.24(\mathrm{~d}, J$ $=8.4 \mathrm{~Hz}, 2 \mathrm{H}), 7.79(\mathrm{ddd}, J=8.3,6.9,1.1 \mathrm{~Hz}, 1 \mathrm{H}), 7.67(\mathrm{ddd}, J=8.2,6.9,1.3 \mathrm{~Hz}, 1 \mathrm{H})$, 3.92 (hept, $J=6.7 \mathrm{~Hz}, 1 \mathrm{H}), 1.43(\mathrm{~d}, J=6.8 \mathrm{~Hz}, 6 \mathrm{H}) ;{ }^{13} \mathrm{C}$ NMR $\left(101 \mathrm{MHz}, \mathrm{CDCl}_{3}\right) \delta$ $165.26,140.53,133.76,130.59,127.70,127.06,126.30,125.07,124.17,30.97$, 22.16; HRMS (ESI) $\mathrm{m} / \mathrm{z}$ calculated for $\mathrm{C}_{12} \mathrm{H}_{13} \mathrm{ClN}\left[(\mathrm{M}+\mathrm{H})^{+}\right]$206.0731, found 206.0731; IR (film) 2971, 2927, 1661, 1557, 1451, 1386, 1245, 1009, 947, 895, $766 \mathrm{~cm}^{-1}$.

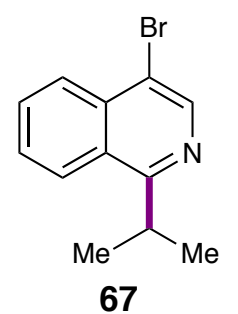

4-Bromo-1-isopropylisoquinoline (67): According to the general procedure A, $\mathrm{FeSO}_{4} \cdot 7 \mathrm{H}_{2} \mathrm{O}(7.0 \mathrm{mg}, 0.025 \mathrm{mmol}, 0.05$ equiv.), picolinic acid $(6.2 \mathrm{mg}, 0.05 \mathrm{mmol}, 0.1$ equiv.), 4-bromoisoquinoline (106.0 $\mathrm{mg}, 0.50 \mathrm{mmol}, 1.0$ equiv.), isobutyric acid (445.0 mg, $5.0 \mathrm{mmol}, 10$ equiv.), $\mathrm{NaBrO}_{3}\left(150.9 \mathrm{mg}, 1.0 \mathrm{mmol}, 2.0\right.$ equiv.), $4.5 \mathrm{~mL}$ of $\mathrm{H}_{2} \mathrm{O}$ and $0.5 \mathrm{~mL}$ of DMSO, $456 \mathrm{~nm}$ LEDs were used. After 24 hours, the reaction mixture was subjected to the workup procedure outlined in the general procedure and purified by flash chromatography ( $5 \%$ ethyl acetate/hexanes) to provide the title compound as a light green oil (71.0 mg, 57\% yield). ${ }^{1} \mathrm{H}$ NMR (400 MHz, $\left.\mathrm{CDCl}_{3}\right) \delta 8.67(\mathrm{~s}, 1 \mathrm{H}), 8.22(\mathrm{~d}, J=8.2 \mathrm{~Hz}$, 1H), 8.19 (d, $J=8.2 \mathrm{~Hz}, 1 \mathrm{H}), 7.77$ (ddd, $J=8.3,6.9,1.2 \mathrm{~Hz}, 1 \mathrm{H}), 7.65$ (ddd, $J=8.3,6.9$, $1.3 \mathrm{~Hz}, 1 \mathrm{H}), 3.92$ (hept, $J=6.8 \mathrm{~Hz}, 1 \mathrm{H}), 1.43(\mathrm{~d}, J=6.8 \mathrm{~Hz}, 6 \mathrm{H}) ;{ }^{13} \mathrm{C}$ NMR $(101 \mathrm{MHz}$, $\left.\mathrm{CDCl}_{3}\right) \delta 165.91,143.43,134.89,130.86,127.81,127.53,126.85,125.10,117.60,30.96$, 22.13; HRMS (ESI) $\mathrm{m} / \mathrm{z}$ calculated for $\mathrm{C}_{12} \mathrm{H}_{13} \mathrm{BrN}\left[(\mathrm{M}+\mathrm{H})^{+}\right]$250.0226, found 250.0226; IR (film) 2964, 1563, 1453, 1386, 1239, 1189, 1064, 1008, 926, 864, $792 \mathrm{~cm}^{-1}$. 


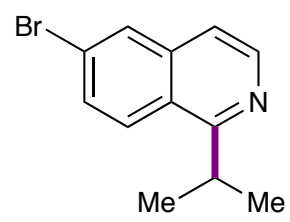

68

6-Bromo-1-isopropylisoquinoline (68): According to the general procedure A, $\mathrm{FeSO}_{4} \cdot 7 \mathrm{H}_{2} \mathrm{O}(7.0 \mathrm{mg}, 0.025 \mathrm{mmol}, 0.05$ equiv.), picolinic acid (6.2 $\mathrm{mg}, 0.05 \mathrm{mmol}, 0.1$ equiv.), 6-bromoisoquinoline (107.0 $\mathrm{mg}, 0.50 \mathrm{mmol}, 1.0$ equiv.), isobutyric acid (445.0 mg, 5.0 mmol, 10 equiv.), $\mathrm{NaBrO}_{3}$ (150.9 mg, 1.0 mmol, 2.0 equiv.), $4.0 \mathrm{~mL}$ of $\mathrm{H}_{2} \mathrm{O}$ and $1.0 \mathrm{~mL}$ of DMSO, $440 \mathrm{~nm}$ LEDs were used. After 24 hours, the reaction mixture was subjected to the workup procedure outlined in the general procedure and purified by flash chromatography (5\% ethyl acetate/hexanes) to provide the title compound as a light yellow oil (81.1 mg, 65\% yield). ${ }^{1} \mathrm{H}$ NMR $\left(400 \mathrm{MHz}, \mathrm{CDCl}_{3}\right) \delta 8.50(\mathrm{~d}, J=5.7 \mathrm{~Hz}, 1 \mathrm{H})$, $8.08(\mathrm{~d}, J=9.0 \mathrm{~Hz}, 1 \mathrm{H}), 7.97(\mathrm{~d}, J=1.9 \mathrm{~Hz}, 1 \mathrm{H}), 7.65(\mathrm{dd}, J=9.0,2.0 \mathrm{~Hz}, 1 \mathrm{H}), 7.39(\mathrm{~d}$, $J=5.7 \mathrm{~Hz}, 1 \mathrm{H}), 3.89$ (hept, $J=6.8 \mathrm{~Hz}, 1 \mathrm{H}), 1.43(\mathrm{~d}, J=6.8 \mathrm{~Hz}, 6 \mathrm{H}) ;{ }^{13} \mathrm{C} \mathrm{NMR}(101$ $\left.\mathrm{MHz}_{\mathrm{CDCl}}\right) \delta 166.52,142.85,137.55,130.37,129.59,126.58,124.59,124.33,117.96$, 31.08, 22.16; HRMS (ESI) m/z calculated for $\mathrm{C}_{12} \mathrm{H}_{13} \mathrm{BrN}\left[(\mathrm{M}+\mathrm{H})^{+}\right] 250.0226$, found 250.0224; IR (film) 2962, 2926, 1611, 1555, 1455, 1239, 1006, 879, 825, $772 \mathrm{~cm}^{-1}$.<smiles>CC(C)c1nc2ccccc2c2ccccc12</smiles>

69

6-Isopropylphenanthridine (69): According to the general procedure $\mathrm{A}, \mathrm{FeSO}_{4} \cdot 7 \mathrm{H}_{2} \mathrm{O}$ (7.0 mg, $0.025 \mathrm{mmol}, 0.05$ equiv.), picolinic acid (6.2 mg, $0.05 \mathrm{mmol}, 0.1$ equiv.), phenanthridine (91.4 mg, $0.50 \mathrm{mmol}, 1.0$ equiv.), isobutyric acid (445.0 mg, $5.0 \mathrm{mmol}$, 
10 equiv.), $\mathrm{NaBrO}_{3}$ (150.9 mg, $1.0 \mathrm{mmol}, 2.0$ equiv.), $4.5 \mathrm{~mL}$ of $\mathrm{H}_{2} \mathrm{O}$ and $0.5 \mathrm{~mL}$ of DMSO, $456 \mathrm{~nm}$ LEDs were used. After 24 hours, the reaction mixture was subjected to the workup procedure outlined in the general procedure and purified by flash chromatography (hexanes) to provide the title compound as a light yellow solid (106.3 mg, 96\% yield). ${ }^{1} \mathrm{H}$ NMR (400 MHz, $\left.\mathrm{CDCl}_{3}\right) \delta 8.65(\mathrm{~d}, J=8.2 \mathrm{~Hz}, 1 \mathrm{H}), 8.54(\mathrm{~d}, J=8.1$ $\mathrm{Hz}, 1 \mathrm{H}), 8.33(\mathrm{~d}, J=8.2 \mathrm{~Hz}, 1 \mathrm{H}), 8.20(\mathrm{~d}, J=8.0 \mathrm{~Hz}, 1 \mathrm{H}), 7.81(\mathrm{t}, J=7.6 \mathrm{~Hz}, 1 \mathrm{H}), 7.73$ (t, $J=7.6 \mathrm{~Hz}, 1 \mathrm{H}), 7.69(\mathrm{t}, J=7.5 \mathrm{~Hz}, 1 \mathrm{H}), 7.62(\mathrm{t}, J=7.5 \mathrm{~Hz}, 1 \mathrm{H}), 4.02$ (hept, $J=6.4$ $\mathrm{Hz}, 1 \mathrm{H}), 1.56(\mathrm{~d}, J=6.8 \mathrm{~Hz}, 6 \mathrm{H}) ;{ }^{13} \mathrm{C} \mathrm{NMR}\left(101 \mathrm{MHz}, \mathrm{CDCl}_{3}\right) \delta 165.77,143.67,132.93$, $129.89,129.83,128.35,127.05,126.12,125.61,124.63,123.35,122.50,121.76,31.43$, 21.91; HRMS (ESI) $\mathrm{m} / \mathrm{z}$ calculated for $\mathrm{C}_{16} \mathrm{H}_{16} \mathrm{~N}\left[(\mathrm{M}+\mathrm{H})^{+}\right]$222.1277, found 222.1276; IR (film) $2963,1582,1485,1381,1267,1083,1006,859,826,757 \mathrm{~cm}^{-1}$. Spectra data are consistent with those reported in the literature: J. Am. Chem. Soc. 2017, 139, 1431514321.<smiles>CC(C)c1ncnc2ccccc12</smiles>

70

4-Isopropylquinazoline (70): According to the general procedure $\mathrm{A}, \mathrm{FeSO}_{4} \cdot 7 \mathrm{H}_{2} \mathrm{O}(7.0$ $\mathrm{mg}, 0.025 \mathrm{mmol}, 0.05$ equiv.), picolinic acid ( $6.2 \mathrm{mg}, 0.05 \mathrm{mmol}, 0.1$ equiv.), quinazoline (66.0 mg, $0.50 \mathrm{mmol}, 1.0$ equiv.), isobutyric acid (445.0 mg, $5.0 \mathrm{mmol}, 10$ equiv.), $\mathrm{NaBrO}_{3}$ (150.9 mg, $1.0 \mathrm{mmol}, 2.0$ equiv.), $4.5 \mathrm{~mL}$ of $\mathrm{H}_{2} \mathrm{O}$ and $0.5 \mathrm{~mL}$ of DMSO, $456 \mathrm{~nm}$ LEDs were used. After 24 hours, the reaction mixture was subjected to the workup procedure outlined in the general procedure and purified by flash chromatography $(5 \%$ ethyl acetate/hexanes) to provide the title compound as a light yellow oil $(62.0 \mathrm{mg}, 72 \%$ 
yield). ${ }^{1} \mathrm{H}$ NMR $\left(400 \mathrm{MHz}, \mathrm{CDCl}_{3}\right) \delta 9.22(\mathrm{~s}, 1 \mathrm{H}), 8.12(\mathrm{~d}, J=8.4 \mathrm{~Hz}, 1 \mathrm{H}), 7.99(\mathrm{~d}, J=$ $8.4 \mathrm{~Hz}, 1 \mathrm{H}), 7.81$ (ddd, $J=8.4,6.9,1.4 \mathrm{~Hz}, 1 \mathrm{H}), 7.57$ (ddd, $J=8.3,6.9,1.3 \mathrm{~Hz}, 1 \mathrm{H}$ ), 3.88 (hept, $J=6.8 \mathrm{~Hz}, 1 \mathrm{H}), 1.39(\mathrm{~d}, J=6.8 \mathrm{~Hz}, 6 \mathrm{H}) ;{ }^{13} \mathrm{C} \mathrm{NMR}\left(101 \mathrm{MHz}, \mathrm{CDCl}_{3}\right) \delta$ $175.72,154.58,149.85,133.20,129.15,127.29,124.04,123.02,30.76,21.58 ;$ HRMS (ESI) $\mathrm{m} / \mathrm{z}$ calculated for $\mathrm{C}_{11} \mathrm{H}_{13} \mathrm{~N}_{2}\left[(\mathrm{M}+\mathrm{H})^{+}\right]$173.1073, found 173.1073; IR (film) 2968, $1579,1396,1376,1318,1193,1155,1100,941,717 \mathrm{~cm}^{-1}$. Spectra data are consistent with those reported in the literature: Angew. Chem. Int. Ed. 2012, 51, 8077-8081.<smiles>CC(C)c1nc(Cl)nc2ccccc12</smiles>
71 2-Chloro-4-isopropylquinazoline (71): According to the general procedure A, $\mathrm{FeSO}_{4} \cdot 7 \mathrm{H}_{2} \mathrm{O}$ (7.0 mg, $0.025 \mathrm{mmol}, 0.05$ equiv.), picolinic acid $(6.2 \mathrm{mg}, 0.05 \mathrm{mmol}, 0.1$ equiv.), 2-Chloroquinazoline ( $83.0 \mathrm{mg}, 0.50 \mathrm{mmol}, 1.0$ equiv.), isobutyric acid (445.0 $\mathrm{mg}$, $5.0 \mathrm{mmol}, 10$ equiv.), $\mathrm{NaBrO}_{3}$ (150.9 mg, $1.0 \mathrm{mmol}, 2.0$ equiv.), $4.5 \mathrm{~mL}$ of $\mathrm{H}_{2} \mathrm{O}$ and 0.5 $\mathrm{mL}$ of DMSO, $456 \mathrm{~nm}$ LEDs were used. After 24 hours, the reaction mixture was subjected to the workup procedure outlined in the general procedure and purified by flash chromatography (5\% ethyl acetate/hexanes) to provide the title compound as a colorless oil (59.0 mg, 57\% yield). ${ }^{1} \mathrm{H}$ NMR (400 MHz, $\left.\mathrm{CDCl}_{3}\right) \delta 8.13(\mathrm{~d}, J=8.4 \mathrm{~Hz}, 1 \mathrm{H}), 7.93(\mathrm{~d}$, $J=8.4 \mathrm{~Hz}, 1 \mathrm{H}), 7.86(\mathrm{ddd}, J=8.4,6.8,1.3 \mathrm{~Hz}, 1 \mathrm{H}), 7.61(\mathrm{ddd}, J=8.3,6.8,1.3 \mathrm{~Hz}, 1 \mathrm{H})$, 3.87 (hept, $J=6.8 \mathrm{~Hz}, 1 \mathrm{H}), 1.41(\mathrm{~d}, J=6.8 \mathrm{~Hz}, 6 \mathrm{H}) ;{ }^{13} \mathrm{C} \mathrm{NMR}\left(101 \mathrm{MHz}, \mathrm{CDCl}_{3}\right) \delta$ 179.76, 157.30, 151.88, 134.41, 128.32, 127.60, 124.40, 121.43, 31.25, 21.50; HRMS (ESI) $\mathrm{m} / \mathrm{z}$ calculated for $\mathrm{C}_{11} \mathrm{H}_{12} \mathrm{ClN}_{2}\left[(\mathrm{M}+\mathrm{H})^{+}\right]$207.0684, found 207.0683; IR (film) 2970, 
$1564,1492,1274,1188,1078,1008,911,868,761 \mathrm{~cm}^{-1}$. Spectra data are consistent with those reported in the literature: Synthesis 2016, 48, 2255-2262.<smiles>CC(C)c1nnc(C(C)C)c2ccccc12</smiles>

72

1,4-Diisopropylphthalazine (72): According to the general procedure $\mathrm{A}, \mathrm{FeSO}_{4} \cdot 7 \mathrm{H}_{2} \mathrm{O}$ (7.0 mg, $0.025 \mathrm{mmol}, 0.05$ equiv.), picolinic acid ( $6.2 \mathrm{mg}, 0.05 \mathrm{mmol}, 0.1$ equiv.), phthalazine (67.0 mg, $0.50 \mathrm{mmol}, 1.0$ equiv.), isobutyric acid (445.0 mg, $5.0 \mathrm{mmol}, 10$ equiv.), $\mathrm{NaBrO}_{3}$ (150.9 mg, $1.0 \mathrm{mmol}, 2.0$ equiv.), $4.0 \mathrm{~mL}$ of $\mathrm{H}_{2} \mathrm{O}$ and $1.0 \mathrm{~mL}$ of DMSO, $440 \mathrm{~nm}$ LEDs were used. After 24 hours, the reaction mixture was subjected to the workup procedure outlined in the general procedure and purified by flash chromatography ( $20 \%$ ethyl acetate/hexanes) to provide the title compound as a light yellow solid (88.1 mg, 82\% yield). ${ }^{1} \mathrm{H}$ NMR (400 MHz, $\left.\mathrm{CDCl}_{3}\right) \delta 8.18(\mathrm{dd}, J=6.3,3.3$ $\mathrm{Hz}, 2 \mathrm{H}), 7.86$ (dd, $J=6.3,3.3 \mathrm{~Hz}, 2 \mathrm{H}), 3.86$ (hept, $J=6.8 \mathrm{~Hz}, 2 \mathrm{H}$ ), $1.52(\mathrm{~d}, J=6.8 \mathrm{~Hz}$, $12 \mathrm{H}) ;{ }^{13} \mathrm{C}$ NMR $\left(101 \mathrm{MHz}, \mathrm{CDCl}_{3}\right) \delta$ 162.54, 131.28, 124.94, 124.27, 30.19, 21.93; HRMS (ESI) $\mathrm{m} / \mathrm{z}$ calculated for $\mathrm{C}_{14} \mathrm{H}_{19} \mathrm{~N}_{2}\left[(\mathrm{M}+\mathrm{H})^{+}\right] 215.1543$, found 215.1542; IR (film) $2965,2930,1538,1467,1318,1187,1086,1033,782,728 \mathrm{~cm}^{-1}$. Spectra data are consistent with those reported in the literature: Org. Lett. 2017, 19, 6594-6597.<smiles>CC(C)c1cnc2ccccc2n1</smiles>

$73 a$<smiles>CC(C)c1nc2ccccc2nc1C(C)C</smiles>

73b 
2-Isopropylquinoxaline (73a) and 2,3-diisopropylquinoxaline (73b): According to the general procedure $\mathrm{A}, \mathrm{FeSO}_{4} \cdot 7 \mathrm{H}_{2} \mathrm{O}(7.0 \mathrm{mg}, 0.025 \mathrm{mmol}, 0.05$ equiv.), picolinic acid (6.2 $\mathrm{mg}, 0.05 \mathrm{mmol}, 0.1$ equiv.), quinoxaline $(66.0 \mathrm{mg}, 0.50 \mathrm{mmol}, 1.0$ equiv.), isobutyric acid (445.0 mg, 5.0 mmol, 10 equiv.), $\mathrm{NaBrO}_{3}$ (150.9 mg, $1.0 \mathrm{mmol}, 2.0$ equiv.), $4.5 \mathrm{~mL}$ of $\mathrm{H}_{2} \mathrm{O}$ and $0.5 \mathrm{~mL}$ of DMSO, $456 \mathrm{~nm}$ LEDs were used. After 24 hours, the reaction mixture was subjected to the workup procedure outlined in the general procedure and purified by flash chromatography ( $5 \%$ ethyl acetate/hexanes) to provide the title compounds as a colorless oil 73a (52.5 mg, 61\% yield) and a white solid 73b (20.0 mg, 19\% yield). 73a: ${ }^{1} \mathrm{H}$ NMR $\left(400 \mathrm{MHz}, \mathrm{CDCl}_{3}\right) \delta 8.76(\mathrm{~s}, 1 \mathrm{H}), 8.09-7.98(\mathrm{~m}, 2 \mathrm{H}), 7.74-$ $7.62(\mathrm{~m}, 2 \mathrm{H}), 3.30$ (hept, $J=6.9 \mathrm{~Hz}, 1 \mathrm{H}), 1.42(\mathrm{~d}, J=7.0 \mathrm{~Hz}, 6 \mathrm{H}) ;{ }^{13} \mathrm{C} \mathrm{NMR}(101 \mathrm{MHz}$, $\left.\mathrm{CDCl}_{3}\right) \delta 161.78,144.69,141.98,141.28,129.73,129.00,128.93,128.80,34.86,21.95$ HRMS (ESI) $\mathrm{m} / \mathrm{z}$ calculated for $\mathrm{C}_{11} \mathrm{H}_{13} \mathrm{~N}_{2}\left[(\mathrm{M}+\mathrm{H})^{+}\right] 173.1073$, found 173.1073; IR (film) $2965,1559,1491,1459,1366,1253,1086,1032,972,921,757 \mathrm{~cm}^{-1}$. Spectra data are consistent with those reported in the literature: Org. Lett. 2014, 16, 4638-4641. 73b: ${ }^{1} \mathrm{H}$ NMR $\left(400 \mathrm{MHz}, \mathrm{CDCl}_{3}\right) \delta 8.00(\mathrm{dd}, J=6.3,3.5 \mathrm{~Hz}, 2 \mathrm{H}), 7.63(\mathrm{dd}, J=6.4,3.4 \mathrm{~Hz}, 2 \mathrm{H})$, 3.54 (hept, $J=6.7 \mathrm{~Hz}, 2 \mathrm{H}), 1.40(\mathrm{~d}, J=6.7 \mathrm{~Hz}, 12 \mathrm{H}) ;{ }^{13} \mathrm{C} \mathrm{NMR}\left(101 \mathrm{MHz}, \mathrm{CDCl}_{3}\right) \delta$ 160.27, 140.91, 128.61, 128.39, 31.19, 22.12; HRMS (ESI) $\mathrm{m} / \mathrm{z}$ calculated for $\mathrm{C}_{14} \mathrm{H}_{19} \mathrm{~N}_{2}$ $\left[(\mathrm{M}+\mathrm{H})^{+}\right]$215.1543, found 215.1542; IR (film) 2964, 1562, 1456, 1380, 1280, 1206, 1094, 1047, 925, $767 \mathrm{~cm}^{-1}$.

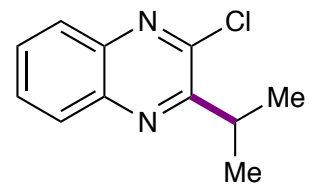


2-Chloro-3-isopropylquinoxaline (74): According to the general procedure A, $\mathrm{FeSO}_{4} \cdot 7 \mathrm{H}_{2} \mathrm{O}$ (7.0 mg, $0.025 \mathrm{mmol}, 0.05$ equiv.), picolinic acid (6.2 mg, $0.05 \mathrm{mmol}, 0.1$ equiv.), 2-chloroquinoxaline ( $81.5 \mathrm{mg}, 0.50 \mathrm{mmol}, 1.0$ equiv.), isobutyric acid (445.0 $\mathrm{mg}$, $5.0 \mathrm{mmol}, 10$ equiv.), $\mathrm{NaBrO}_{3}$ (150.9 mg, $1.0 \mathrm{mmol}, 2.0$ equiv.), $4.5 \mathrm{~mL}$ of $\mathrm{H}_{2} \mathrm{O}$ and 0.5 $\mathrm{mL}$ of DMSO, $456 \mathrm{~nm}$ LEDs were used. After 24 hours, the reaction mixture was subjected to the workup procedure outlined in the general procedure and purified by flash chromatography ( $2 \%$ ethyl acetate/hexanes) to provide the title compound as a white solid (87.8 mg, 86\% yield). ${ }^{1} \mathrm{H}$ NMR (400 MHz, $\left.\mathrm{CDCl}_{3}\right) \delta 8.08-8.01(\mathrm{~m}, 1 \mathrm{H}), 7.99-$ $7.92(\mathrm{~m}, 1 \mathrm{H}), 7.76-7.64(\mathrm{~m}, 2 \mathrm{H}), 3.70$ (hept, $J=6.8 \mathrm{~Hz}, 1 \mathrm{H}), 1.40(\mathrm{~d}, J=6.8 \mathrm{~Hz}, 6 \mathrm{H})$; ${ }^{13} \mathrm{C}$ NMR $\left(101 \mathrm{MHz}, \mathrm{CDCl}_{3}\right) \delta 159.81,147.28,141.02,140.64,129.82,129.78,128.74$, 127.96, 32.55, 20.95; HRMS (ESI) m/z calculated for $\mathrm{C}_{11} \mathrm{H}_{12} \mathrm{ClN}_{2}\left[(\mathrm{M}+\mathrm{H})^{+}\right]$207.0684, found 207.0683; IR (film) 2965, 1561, 1483, 1265, 1194, 1131, 1019, 874, $765 \mathrm{~cm}^{-1}$.

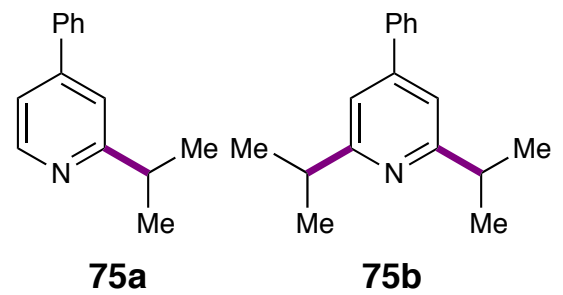

\section{2-Isopropyl-4-phenylpyridine (75a) and 2,6-diisopropyl-4-phenylpyridine (75b):}

According to the general procedure $\mathrm{A}, \mathrm{FeSO}_{4} \cdot 7 \mathrm{H}_{2} \mathrm{O}(7.0 \mathrm{mg}, 0.025 \mathrm{mmol}, 0.05$ equiv.), picolinic acid (6.2 mg, $0.05 \mathrm{mmol}, 0.1$ equiv.), 4-phenylpyridine (79.0 mg, $0.50 \mathrm{mmol}$, 1.0 equiv.), isobutyric acid (445.0 mg, $5.0 \mathrm{mmol}, 10$ equiv.), $\mathrm{NaBrO}_{3}$ (150.9 mg, 1.0 mmol, 2.0 equiv.), $4.5 \mathrm{~mL}$ of $\mathrm{H}_{2} \mathrm{O}$ and $0.5 \mathrm{~mL}$ of DMSO, $456 \mathrm{~nm}$ LEDs were used. After 24 hours, the reaction mixture was subjected to the workup procedure outlined in the general procedure and purified by flash chromatography (5\% ethyl acetate/hexanes) to provide the title compounds as a colorless oil 75a (40.0 mg, 40\% yield) and a colorless 
oil 75b (23.4 mg, 20\% yield). 75a: ${ }^{1} \mathrm{H}$ NMR (400 MHz, $\left.\mathrm{CDCl}_{3}\right) \delta 8.58(\mathrm{~d}, J=5.2 \mathrm{~Hz}$, 1H), $7.68-7.60(\mathrm{~m}, 2 \mathrm{H}), 7.51-7.40(\mathrm{~m}, 3 \mathrm{H}), 7.38(\mathrm{~s}, 1 \mathrm{H}), 7.33(\mathrm{dd}, J=5.2,1.7 \mathrm{~Hz}, 1 \mathrm{H})$, 3.14 (hept, $J=6.9 \mathrm{~Hz}, 1 \mathrm{H}), 1.36(\mathrm{~d}, J=6.9 \mathrm{~Hz}, 6 \mathrm{H}) ;{ }^{13} \mathrm{C} \mathrm{NMR}\left(101 \mathrm{MHz}, \mathrm{CDCl}_{3}\right) \delta$ $167.67,149.24,149.01,138.63,128.98,128.84,127.02,119.22,118.70,36.33,22.58$; HRMS (ESI) m/z calculated for $\mathrm{C}_{14} \mathrm{H}_{16} \mathrm{~N}\left[(\mathrm{M}+\mathrm{H})^{+}\right]$198.1277, found 198.1277; IR (film) $2961,1595,1546,1474,1398,1289,1056,886,841,759 \mathrm{~cm}^{-1}$. Spectra data are consistent with those reported in the literature: J. Am. Chem. Soc. 2017, 139, 1431514321. 75b: ${ }^{1} \mathrm{H}$ NMR (400 MHz, $\left.\mathrm{CDCl}_{3}\right) \delta 7.66-7.60(\mathrm{~m}, 2 \mathrm{H}), 7.51-7.44(\mathrm{~m}, 2 \mathrm{H})$, $7.44-7.39(\mathrm{~m}, 1 \mathrm{H}), 7.19(\mathrm{~s}, 2 \mathrm{H}), 3.13(\mathrm{hept}, J=6.8 \mathrm{~Hz}, 2 \mathrm{H}), 1.35(\mathrm{~d}, J=6.9 \mathrm{~Hz}, 12 \mathrm{H})$ ${ }^{13} \mathrm{C} \mathrm{NMR}\left(101 \mathrm{MHz}, \mathrm{CDCl}_{3}\right) \delta 166.92,149.32,139.50,128.88,128.53,127.12,115.65$, 36.38, 22.75; HRMS (ESI) m/z calculated for $\mathrm{C}_{17} \mathrm{H}_{22} \mathrm{~N}\left[(\mathrm{M}+\mathrm{H})^{+}\right] 240.1747$, found 240.1746; IR (film) 2960, 1596, 1553, 1468, 1405, 1359, 1155, 1052, 872, $760 \mathrm{~cm}^{-1}$.

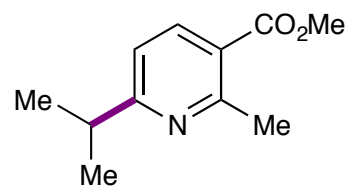

76

Methyl 6-isopropyl-2-methylnicotinate (76): According to the general procedure A, $\mathrm{FeSO}_{4} \cdot 7 \mathrm{H}_{2} \mathrm{O}(7.0 \mathrm{mg}, 0.025 \mathrm{mmol}, 0.05$ equiv.), picolinic acid (6.2 mg, $0.05 \mathrm{mmol}, 0.1$ equiv.), methyl 2-methylnicotinate $(78.0 \mathrm{mg}, 0.50 \mathrm{mmol}, 1.0$ equiv.), isobutyric acid (445.0 mg, 5.0 mmol, 10 equiv.), $\mathrm{NaBrO}_{3}$ (150.9 mg, $1.0 \mathrm{mmol}, 2.0$ equiv.), $4.5 \mathrm{~mL}$ of $\mathrm{H}_{2} \mathrm{O}$ and $0.5 \mathrm{~mL}$ of DMSO, $456 \mathrm{~nm}$ LEDs were used. After 24 hours, the reaction mixture was subjected to the workup procedure outlined in the general procedure and purified by flash chromatography ( $33 \%$ ethyl acetate/hexanes) to provide the title compound as a colorless oil (58.5 mg, 61\% yield). ${ }^{1} \mathrm{H}$ NMR (400 MHz, $\left.\mathrm{CDCl}_{3}\right) \delta 8.12$ (d, 
$J=8.1 \mathrm{~Hz}, 1 \mathrm{H}), 7.08(\mathrm{~d}, J=8.1 \mathrm{~Hz}, 1 \mathrm{H}), 3.90(\mathrm{~s}, 3 \mathrm{H}), 3.08$ (hept, $J=6.5 \mathrm{~Hz}, 1 \mathrm{H}), 2.82$

(s, 3H), $1.29(\mathrm{~d}, J=6.9 \mathrm{~Hz}, 6 \mathrm{H}) ;{ }^{13} \mathrm{C} \mathrm{NMR}\left(101 \mathrm{MHz}, \mathrm{CDCl}_{3}\right) \delta 170.20,167.08,159.22$, 139.14, 122.67, 117.39, 52.06, 36.38, 24.74, 22.36; HRMS (ESI) m/z calculated for $\mathrm{C}_{11} \mathrm{H}_{16} \mathrm{NO}_{2}\left[(\mathrm{M}+\mathrm{H})^{+}\right]$194.1176, found 194.1176; IR (film) 2963, 1723, 1587, 1434, 1271, $1202,1152,1079,845,789,741 \mathrm{~cm}^{-1}$.

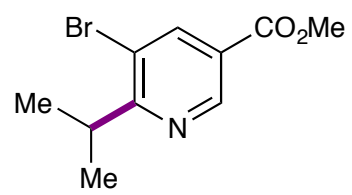

77

Methyl 5-bromo-6-isopropylnicotinate (77): According to the general procedure A, $\mathrm{FeSO}_{4} \cdot 7 \mathrm{H}_{2} \mathrm{O}$ (7.0 mg, $0.025 \mathrm{mmol}, 0.05$ equiv.), picolinic acid (6.2 mg, $0.05 \mathrm{mmol}, 0.1$ equiv.), methyl 5-bromonicotinate (110.0 $\mathrm{mg}, 0.50 \mathrm{mmol}, 1.0$ equiv.), isobutyric acid (445.0 mg, 5.0 mmol, 10 equiv.), $\mathrm{NaBrO}_{3}$ (150.9 mg, $1.0 \mathrm{mmol}, 2.0$ equiv.), $4.0 \mathrm{~mL}$ of $\mathrm{H}_{2} \mathrm{O}$ and $1.0 \mathrm{~mL}$ of DMSO, $440 \mathrm{~nm}$ LEDs were used. After 24 hours, the reaction mixture was subjected to the workup procedure outlined in the general procedure and purified by flash chromatography ( $5 \%$ ethyl acetate/hexanes) to provide the title compound as a colorless oil (91.3 mg, 71\% yield). ${ }^{1} \mathrm{H}$ NMR (400 MHz, $\left.\mathrm{CDCl}_{3}\right) \delta 9.04(\mathrm{~d}$, $J=1.8 \mathrm{~Hz}, 1 \mathrm{H}), 8.37(\mathrm{~d}, J=1.9 \mathrm{~Hz}, 1 \mathrm{H}), 3.92(\mathrm{~s}, 3 \mathrm{H}), 3.58$ (hept, $J=6.7 \mathrm{~Hz}, 1 \mathrm{H}), 1.27$ $(\mathrm{d}, J=6.8 \mathrm{~Hz}, 6 \mathrm{H}) ;{ }^{13} \mathrm{C} \mathrm{NMR}\left(101 \mathrm{MHz}, \mathrm{CDCl}_{3}\right) \delta 169.07,164.68,148.74,140.90$, 124.77, 120.36, 52.45, 34.20, 21.08; HRMS (ESI) m/z calculated for $\mathrm{C}_{10} \mathrm{H}_{13} \mathrm{BrNO}_{2}$ $\left[(\mathrm{M}+\mathrm{H})^{+}\right]$258.0124, found 258.0124; IR (film) 2966, 1727, 1587, 1431, 1271, 1119, $1041,969,850,775 \mathrm{~cm}^{-1}$. Spectra data are consistent with those reported in the literature: Org. Lett. 2016, 18, 3738-3741. 


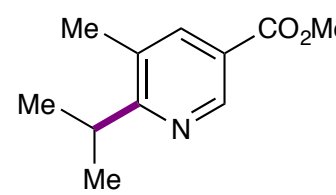

$78 a$

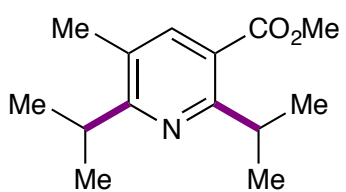

$78 b$

Methyl 6-isopropyl-5-methylnicotinate (78a) and methyl 2,6-diisopropyl-5-

methylnicotinate (78b): According to the general procedure $\mathrm{A}, \mathrm{FeSO}_{4} \cdot 7 \mathrm{H}_{2} \mathrm{O}(7.0 \mathrm{mg}$, $0.025 \mathrm{mmol}, 0.05$ equiv.), picolinic acid (6.2 mg, $0.05 \mathrm{mmol}, 0.1$ equiv.), methyl $5-$ methylnicotinate (77.5 mg, $0.50 \mathrm{mmol}, 1.0$ equiv.), isobutyric acid (445.0 mg, $5.0 \mathrm{mmol}$, 10 equiv.), $\mathrm{NaBrO}_{3}$ (150.9 mg, $1.0 \mathrm{mmol}, 2.0$ equiv.), $4.0 \mathrm{~mL}$ of $\mathrm{H}_{2} \mathrm{O}$ and $1.0 \mathrm{~mL}$ of DMSO, $440 \mathrm{~nm}$ LEDs were used. After 24 hours, the reaction mixture was subjected to the workup procedure outlined in the general procedure and purified by flash chromatography ( $5 \%$ ethyl acetate/hexanes) to provide the title compounds as a colorless oil 78a (44.6 mg, 46\% yield) and a colorless oil 78b (25.4 mg, 22\% yield). 78a: ${ }^{1} \mathrm{H}$ NMR $\left(400 \mathrm{MHz}, \mathrm{CDCl}_{3}\right) \delta 8.97(\mathrm{~d}, J=1.8 \mathrm{~Hz}, 1 \mathrm{H}), 7.97(\mathrm{~d}, J=1.8 \mathrm{~Hz}, 1 \mathrm{H}), 3.89(\mathrm{~s}, 3 \mathrm{H}), 3.26$ (hept, $J=6.8 \mathrm{~Hz}, 1 \mathrm{H}), 2.35(\mathrm{~s}, 3 \mathrm{H}), 1.25(\mathrm{~d}, J=6.8 \mathrm{~Hz}, 6 \mathrm{H}) ;{ }^{13} \mathrm{C}$ NMR $(101 \mathrm{MHz}$, $\left.\mathrm{CDCl}_{3}\right) \delta 169.55,166.15,147.91,138.37,129.75,123.18,52.06,31.62,21.33,18.49$; HRMS (ESI) m/z calculated for $\mathrm{C}_{11} \mathrm{H}_{16} \mathrm{NO}_{2}\left[(\mathrm{M}+\mathrm{H})^{+}\right]$194.1176, found 194.1175; IR (film) 2961, 1722, 1600, 1437, 1408, 1294, 1259, 1222, 1005, $785 \mathrm{~cm}^{-1}$. 78b: ${ }^{1} \mathrm{H}$ NMR $\left(400 \mathrm{MHz}, \mathrm{CDCl}_{3}\right) \delta 7.76(\mathrm{~s}, 1 \mathrm{H}), 3.88(\mathrm{~s}, 3 \mathrm{H}), 3.80$ (hept, $\left.J=6.7 \mathrm{~Hz}, 1 \mathrm{H}\right), 3.22$ (hept, $J$ $=6.7 \mathrm{~Hz}, 1 \mathrm{H}), 2.29(\mathrm{~s}, 3 \mathrm{H}), 1.26(\mathrm{~d}, J=6.4 \mathrm{~Hz}, 6 \mathrm{H}), 1.25(\mathrm{~d}, J=6.3 \mathrm{~Hz}, 6 \mathrm{H}) ;{ }^{13} \mathrm{C} \mathrm{NMR}$ $\left(101 \mathrm{MHz}, \mathrm{CDCl}_{3}\right) \delta 167.90,167.22,164.06,139.16,126.16,121.33,51.95,32.05,31.81$, 22.39, 21.46, 17.75; HRMS (ESI) m/z calculated for $\mathrm{C}_{14} \mathrm{H}_{22} \mathrm{NO}_{2}\left[(\mathrm{M}+\mathrm{H})^{+}\right] 236.1645$, found 236.1644; IR (film) 2961, 1722, 1596, 1550, 1430, 1279, 1235, 1113, 1029, 943 , $807 \mathrm{~cm}^{-1}$. 


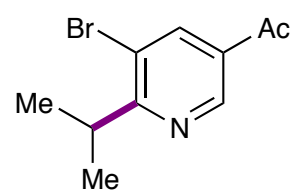

79

1-(5-Bromo-6-isopropylpyridin-3-yl)ethan-1-one (79): According to the general procedure $\mathrm{A}, \mathrm{FeSO}_{4} \cdot 7 \mathrm{H}_{2} \mathrm{O}(7.0 \mathrm{mg}, 0.025 \mathrm{mmol}, 0.05$ equiv.), picolinic acid (6.2 $\mathrm{mg}$, $0.05 \mathrm{mmol}, 0.1$ equiv.), 1-(5-bromopyridine-3-yl)ethanone (103.0 mg, $0.50 \mathrm{mmol}, 1.0$ equiv.), isobutyric acid (445.0 mg, 5.0 mmol, 10 equiv.), $\mathrm{NaBrO}_{3}(150.9 \mathrm{mg}, 1.0 \mathrm{mmol}$, 2.0 equiv.), $4.5 \mathrm{~mL}$ of $\mathrm{H}_{2} \mathrm{O}$ and $0.5 \mathrm{~mL}$ of DMSO, $456 \mathrm{~nm}$ LEDs were used. After 24 hours, the reaction mixture was subjected to the workup procedure outlined in the general procedure and purified by flash chromatography (10\% ethyl acetate/hexanes) to provide the title compound as a colorless oil (76.5 mg, 63\% yield). ${ }^{1} \mathrm{H}$ NMR (400 $\left.\mathrm{MHz}, \mathrm{CDCl}_{3}\right) \delta$ $9.00(\mathrm{~d}, J=2.0 \mathrm{~Hz}, 1 \mathrm{H}), 8.31(\mathrm{~d}, J=2.0 \mathrm{~Hz}, 1 \mathrm{H}), 3.59$ (hept, $J=6.7 \mathrm{~Hz}, 1 \mathrm{H}), 2.59$ (s, 3H), $1.27(\mathrm{~d}, J=6.8 \mathrm{~Hz}, 6 \mathrm{H}) ;{ }^{13} \mathrm{C} \mathrm{NMR}\left(101 \mathrm{MHz}, \mathrm{CDCl}_{3}\right) \delta$ 195.28, 169.22, 147.77, 139.47, 131.24, 121.09, 34.25, 26.67, 21.08; HRMS (ESI) m/z calculated for $\mathrm{C}_{10} \mathrm{H}_{13} \mathrm{BrNO}\left[(\mathrm{M}+\mathrm{H})^{+}\right] 242.0175$, found 242.0175; IR (film) 2967, 1688, 1580, 1383, $1357,1283,1249,1037,915,828,769 \mathrm{~cm}^{-1}$.<smiles>CC(C)c1nc(C#N)ncc1Br</smiles>

$80 a$<smiles>CC(C)c1nc(C#N)nc(C(C)C)c1Br</smiles>

$80 b$

\section{5-Bromo-4-isopropylpyrimidine-2-carbonitrile (80a) and 5-bromo-4,6-}

diisopropylpyrimidine-2-carbonitrile (80b): According to the general procedure A, $\mathrm{FeSO}_{4} \cdot 7 \mathrm{H}_{2} \mathrm{O}(7.0 \mathrm{mg}, 0.025 \mathrm{mmol}, 0.05$ equiv.), picolinic acid (6.2 mg, $0.05 \mathrm{mmol}, 0.1$ 
equiv.), 5-bromopyrimidine-2-carbonitrile ( $95.0 \mathrm{mg}, 0.50 \mathrm{mmol}, 1.0$ equiv.), isobutyric acid (445.0 mg, 5.0 mmol, 10 equiv.), $\mathrm{NaBrO}_{3}(150.9 \mathrm{mg}, 1.0 \mathrm{mmol}, 2.0$ equiv.), $4.5 \mathrm{~mL}$ of $\mathrm{H}_{2} \mathrm{O}$ and $0.5 \mathrm{~mL}$ of DMSO, $456 \mathrm{~nm}$ LEDs were used. After 24 hours, the reaction mixture was subjected to the workup procedure outlined in the general procedure and purified by flash chromatography ( $5 \%$ ethyl acetate/hexanes) to provide the title compounds as a colorless oil $\mathbf{8 0 a}(80.4 \mathrm{mg}, 71 \%$ yield) and a colorless oil $\mathbf{8 0 b}(16.2 \mathrm{mg}$, $12 \%$ yield). 80a: ${ }^{1} \mathrm{H}$ NMR (400 MHz, $\left.\mathrm{CDCl}_{3}\right) \delta 8.79(\mathrm{~s}, 1 \mathrm{H}), 3.50$ (hept, $J=6.8 \mathrm{~Hz}, 1 \mathrm{H}$ ), $1.30(\mathrm{~d}, J=6.8 \mathrm{~Hz}, 6 \mathrm{H}) ;{ }^{13} \mathrm{C} \mathrm{NMR}\left(101 \mathrm{MHz}, \mathrm{CDCl}_{3}\right) \delta 175.27,159.34,143.04,123.82$, 115.37, 34.20, 20.36; HRMS (ESI) m/z calculated for $\mathrm{C}_{8} \mathrm{H}_{8} \mathrm{BrN}_{3}\left[\mathrm{M}^{+}\right]$224.9902, found 224.9907; IR (film) 2968, 1579, 1465, 1396, 1376, 1318, 1193, 1100, 941, 897, $717 \mathrm{~cm}^{-1}$. 80b: ${ }^{1} \mathrm{H}$ NMR $\left(400 \mathrm{MHz}, \mathrm{CDCl}_{3}\right) \delta 3.58$ (hept, $\left.J=6.7 \mathrm{~Hz}, 2 \mathrm{H}\right), 1.28(\mathrm{~d}, J=6.7 \mathrm{~Hz}, 12 \mathrm{H})$; ${ }^{13} \mathrm{C}$ NMR $\left(101 \mathrm{MHz}, \mathrm{CDCl}_{3}\right) \delta 174.88,142.67,123.84,115.74,34.59,20.63$; HRMS (ESI) $\mathrm{m} / \mathrm{z}$ calculated for $\mathrm{C}_{11} \mathrm{H}_{14} \mathrm{BrN}_{3}\left[\mathrm{M}^{+}\right]$267.0371, found 267.0377; IR (film) 2972, $1736,1572,1563,1372,1237,1085,1044,915,854 \mathrm{~cm}^{-1}$.<smiles>COc1ncc(Br)c(C(C)C)n1</smiles>

81a

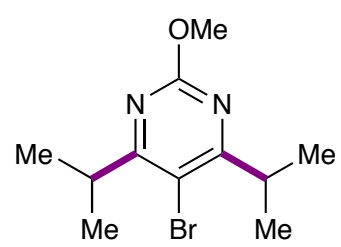

81b

\section{5-Bromo-4-isopropyl-2-methoxypyrimidine (81a) and 5-bromo-4,6-diisopropyl-2-}

methoxypyrimidine (81b): According to the general procedure A, $\mathrm{FeSO}_{4} \cdot 7 \mathrm{H}_{2} \mathrm{O}(7.0 \mathrm{mg}$, $0.025 \mathrm{mmol}, 0.05$ equiv.), picolinic acid ( $6.2 \mathrm{mg}, 0.05 \mathrm{mmol}, 0.1$ equiv.), 5-bromo-2methoxypyrimidine ( $96.5 \mathrm{mg}, 0.50 \mathrm{mmol}, 1.0$ equiv.), isobutyric acid (445.0 mg, 5.0 mmol, 10 equiv.), $\mathrm{NaBrO}_{3}$ (150.9 mg, $1.0 \mathrm{mmol}, 2.0$ equiv.), $4.0 \mathrm{~mL}$ of $\mathrm{H}_{2} \mathrm{O}$ and $1.0 \mathrm{~mL}$ of DMSO, $440 \mathrm{~nm}$ LEDs were used. After 24 hours, the reaction mixture was subjected 
to the workup procedure outlined in the general procedure and purified by flash chromatography (5\% ethyl acetate/hexanes) to provide the title compounds as a colorless oil 81a (55.3 mg, 48\% yield) and a colorless oil $81 \mathrm{~b}$ ( $32.7 \mathrm{mg}, 24 \%$ yield). 81a: ${ }^{1} \mathrm{H}$ NMR $\left(400 \mathrm{MHz}, \mathrm{CDCl}_{3}\right) \delta 8.43(\mathrm{~s}, 1 \mathrm{H}), 3.98(\mathrm{~s}, 3 \mathrm{H}), 3.38$ (hept, $\left.J=6.7 \mathrm{~Hz}, 1 \mathrm{H}\right), 1.25(\mathrm{~d}, J=$ $6.8 \mathrm{~Hz}, 6 \mathrm{H}) ;{ }^{13} \mathrm{C}$ NMR $\left(101 \mathrm{MHz}, \mathrm{CDCl}_{3}\right) \delta 175.04,164.43,160.02,111.88,55.10,33.82$, 20.41; HRMS (ESI) $\mathrm{m} / \mathrm{z}$ calculated for $\mathrm{C}_{8} \mathrm{H}_{12} \mathrm{BrN}_{2} \mathrm{O}\left[(\mathrm{M}+\mathrm{H})^{+}\right]$231.0128, found 231.0127; IR (film) 2969, 1560, 1530, 1460, 1380, 1360, 1311, 1046, 1018, 935, $797 \mathrm{~cm}^{-1}$. Spectra data are consistent with those reported in the literature: Org. Biomol. Chem. 2015, 13, 2750-2755. 81b: ${ }^{1} \mathrm{H}$ NMR (400 MHz, $\left.\mathrm{CDCl}_{3}\right) \delta 3.99$ (s, 3H), 3.51 (hept, $J=6.7 \mathrm{~Hz}, 2 \mathrm{H}$ ), $1.25(\mathrm{~d}, J=6.7 \mathrm{~Hz}, 12 \mathrm{H}) ;{ }^{13} \mathrm{C} \mathrm{NMR}\left(101 \mathrm{MHz}, \mathrm{CDCl}_{3}\right) \delta 174.96,163.93,112.07,54.80$, 34.19, 20.72; HRMS (ESI) m/z calculated for $\mathrm{C}_{11} \mathrm{H}_{18} \mathrm{BrN}_{2} \mathrm{O}\left[(\mathrm{M}+\mathrm{H})^{+}\right] 273.0597$, found 273.0596; IR (film) 2967, 1533, 1457, 1352, 1272, 1203, 1127, 1049, 1020, $805 \mathrm{~cm}^{-1}$.

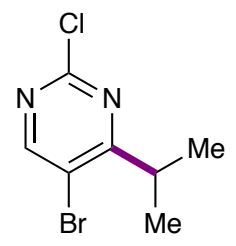

$82 a$

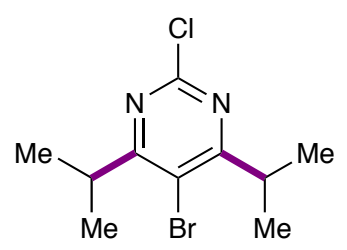

82b

\section{5-Bromo-2-chloro-4-isopropylpyrimidine (82a) and 5-bromo-2-chloro-4,6-}

diisopropylpyrimidine (82b): According to the general procedure $\mathrm{A}, \mathrm{FeSO}_{4} \cdot 7 \mathrm{H}_{2} \mathrm{O}(7.0$ $\mathrm{mg}, 0.025 \mathrm{mmol}, 0.05$ equiv.), picolinic acid (6.2 mg, $0.05 \mathrm{mmol}, 0.1$ equiv.), 5-bromo-2chloropyrimidine ( $98.5 \mathrm{mg}, 0.50 \mathrm{mmol}, 1.0$ equiv.), isobutyric acid (445.0 mg, $5.0 \mathrm{mmol}$, 10 equiv.), $\mathrm{NaBrO}_{3}$ (150.9 mg, $1.0 \mathrm{mmol}, 2.0$ equiv.), $4.0 \mathrm{~mL}$ of $\mathrm{H}_{2} \mathrm{O}$ and $1.0 \mathrm{~mL}$ of DMSO, $440 \mathrm{~nm}$ LEDs were used. After 24 hours, the reaction mixture was subjected to the workup procedure outlined in the general procedure and purified by flash chromatography (5\% ethyl acetate/hexanes) to provide the title compounds as a colorless 
oil 82a (48.4 mg, 41\% yield) and a white solid 82b (20.0 mg, 15\% yield). 82a: ${ }^{1} \mathrm{H}$ NMR $\left(400 \mathrm{MHz}, \mathrm{CDCl}_{3}\right) \delta 8.56(\mathrm{~s}, 1 \mathrm{H}), 3.43$ (hept, $\left.J=6.7 \mathrm{~Hz}, 1 \mathrm{H}\right), 1.28(\mathrm{~d}, J=6.8 \mathrm{~Hz}, 6 \mathrm{H})$; ${ }^{13} \mathrm{C}$ NMR (101 MHz, $\left.\mathrm{CDCl}_{3}\right) \delta 176.33,160.45,159.74,118.77,34.12,20.36$; HRMS (ESI) $\mathrm{m} / \mathrm{z}$ calculated for $\mathrm{C}_{7} \mathrm{H}_{9} \mathrm{BrClN}_{2}\left[(\mathrm{M}+\mathrm{H})^{+}\right]$234.9632, found 234.9631; IR (film) $2971,1543,1513,1378,1319,1187,1079,1021,906,844,783 \mathrm{~cm}^{-1}$. Spectra data are consistent with those reported in the literature: Org. Biomol. Chem. 2015, 13, 2750-2755. 82b: ${ }^{1} \mathrm{H}$ NMR (400 MHz, $\left.\mathrm{CDCl}_{3}\right) \delta 3.54$ (hept, $\left.J=6.6 \mathrm{~Hz}, 2 \mathrm{H}\right), 1.27(\mathrm{~d}, J=6.8 \mathrm{~Hz}, 12 \mathrm{H})$; ${ }^{13} \mathrm{C}$ NMR $\left(101 \mathrm{MHz}, \mathrm{CDCl}_{3}\right) \delta 176.13,159.46,118.92,34.45,20.65 ; \mathrm{HRMS}(\mathrm{ESI}) \mathrm{m} / \mathrm{z}$ calculated for $\mathrm{C}_{10} \mathrm{H}_{15} \mathrm{BrClN}_{2}\left[(\mathrm{M}+\mathrm{H})^{+}\right]$277.0102, found 277.0102; IR (film) 2969, 1542, $1498,1372,1247,1079,1014,900,857,797 \mathrm{~cm}^{-1}$.

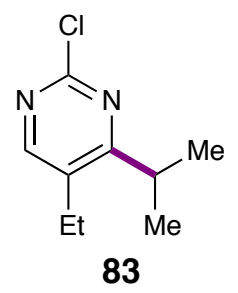

2-Chloro-5-ethyl-4-isopropylpyrimidine (83): According to the general procedure A, $\mathrm{FeSO}_{4} \cdot 7 \mathrm{H}_{2} \mathrm{O}$ (7.0 mg, $0.025 \mathrm{mmol}, 0.05$ equiv.), picolinic acid ( $6.2 \mathrm{mg}, 0.05 \mathrm{mmol}, 0.1$ equiv.), 2-chloro-5-ethylpyrimidine ( $73.5 \mathrm{mg}, 0.50 \mathrm{mmol}, 1.0$ equiv.), isobutyric acid (445.0 mg, $5.0 \mathrm{mmol}, 10$ equiv.), $\mathrm{NaBrO}_{3}(150.9 \mathrm{mg}, 1.0 \mathrm{mmol}, 2.0$ equiv.), $4.0 \mathrm{~mL}$ of $\mathrm{H}_{2} \mathrm{O}$ and $1.0 \mathrm{~mL}$ of DMSO, $440 \mathrm{~nm}$ LEDs were used. After 24 hours, the reaction mixture was subjected to the workup procedure outlined in the general procedure and purified by flash chromatography ( $10 \%$ ethyl acetate/hexanes) to provide the title compound as colorless oil (44.1 mg, 48\% yield). ${ }^{1} \mathrm{H}$ NMR (400 MHz, $\left.\mathrm{CDCl}_{3}\right) \delta 8.30$ (s, 1H), 3.20 (hept, $J=6.8 \mathrm{~Hz}, 1 \mathrm{H}), 2.65$ (q, $J=7.6 \mathrm{~Hz}, 2 \mathrm{H}), 1.27$ (d, $J=6.7 \mathrm{~Hz}, 6 \mathrm{H}), 1.24$ $(\mathrm{t}, J=7.6 \mathrm{~Hz}, 3 \mathrm{H}) ;{ }^{13} \mathrm{C} \mathrm{NMR}\left(101 \mathrm{MHz}, \mathrm{CDCl}_{3}\right) \delta 176.73,159.07,158.89,131.83,31.06$, 
21.94, 21.46, 14.87; HRMS (ESI) m/z calculated for $\mathrm{C}_{9} \mathrm{H}_{14} \mathrm{ClN}_{2}\left[(\mathrm{M}+\mathrm{H})^{+}\right] 185.0840$, found 185.0839; IR (film) 2968, 1566, 1538, 1410, 1377, 1342, 1202, 1161, 1072, 908, $856 \mathrm{~cm}^{-1}$. Spectra data are consistent with those reported in the literature: Org. Biomol. Chem. 2015, 13, 2750-2755.

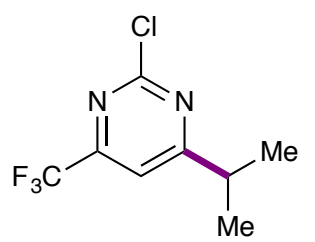

84

2-Chloro-4-isopropyl-6-(trifluoromethyl)pyrimidine (84): According to the general procedure $\mathrm{A}, \mathrm{FeSO}_{4} \cdot 7 \mathrm{H}_{2} \mathrm{O}(7.0 \mathrm{mg}, 0.025 \mathrm{mmol}, 0.05$ equiv.), picolinic acid (6.2 $\mathrm{mg}$, $0.05 \mathrm{mmol}, 0.1$ equiv.), 2-chloro-4-(trifluoromethyl)pyrimidine (92.0 mg, $0.50 \mathrm{mmol}, 1.0$ equiv.), isobutyric acid (445.0 mg, 5.0 mmol, 10 equiv.), $\mathrm{NaBrO}_{3}(150.9 \mathrm{mg}, 1.0 \mathrm{mmol}$, 2.0 equiv.), $4.5 \mathrm{~mL}$ of $\mathrm{H}_{2} \mathrm{O}$ and $0.5 \mathrm{~mL}$ of DMSO, $456 \mathrm{~nm}$ LEDs were used. After 24 hours, the reaction mixture was subjected to the workup procedure outlined in the general procedure and purified by flash chromatography ( $5 \%$ ethyl acetate/hexanes) to provide the title compound as a colorless oil $(60.0 \mathrm{mg}, 54 \%$ yield $) .{ }^{1} \mathrm{H} \mathrm{NMR}\left(400 \mathrm{MHz}, \mathrm{CDCl}_{3}\right) \delta$ $7.44(\mathrm{~s}, 1 \mathrm{H}), 3.13$ (hept, $J=6.9 \mathrm{~Hz}, 1 \mathrm{H}), 1.35(\mathrm{~d}, J=6.9 \mathrm{~Hz}, 6 \mathrm{H}) ;{ }^{13} \mathrm{C} \mathrm{NMR}(101 \mathrm{MHz}$, $\left.\mathrm{CDCl}_{3}\right) \delta 182.53,161.77,157.83(\mathrm{q}), 119.82(\mathrm{q}), 112.76$ (q), 36.48, 21.42; HRMS (ESI) $\mathrm{m} / \mathrm{z}$ calculated for $\mathrm{C}_{8} \mathrm{H}_{9} \mathrm{ClF}_{3} \mathrm{~N}_{2}\left[(\mathrm{M}+\mathrm{H})^{+}\right]$225.0401, found 225.0399; IR (film) 2975, $1582,1542,1378,1327,1262,1148,1118,917,849,707 \mathrm{~cm}^{-1}$. Spectra data are consistent with those reported in the literature: Org. Biomol. Chem. 2015, 13, 2750-2755. 
<smiles>CC(C)c1cc(Cl)nc(Cl)n1</smiles>

85

2,4-Dichloro-6-isopropylpyrimidine (85): According to the general procedure B, $\mathrm{FeSO}_{4} \cdot 7 \mathrm{H}_{2} \mathrm{O}$ (7.0 mg, $0.025 \mathrm{mmol}, 0.05$ equiv.), picolinic acid (6.2 mg, $0.05 \mathrm{mmol}, 0.1$ equiv.), 2,4-dichloropyrimidine (76.0 mg, $0.50 \mathrm{mmol}, 1.0$ equiv.), isobutyric acid (445.0 mg, 5.0 mmol, 10 equiv.), $\mathrm{NaClO}_{3}$ (106.5 mg, 1.0 mmol, 2.0 equiv.), $4.0 \mathrm{~mL}$ of $\mathrm{H}_{2} \mathrm{O}$ and $1.0 \mathrm{~mL}$ of DMSO, $427 \mathrm{~nm}$ LEDs were used. After 36 hours, the reaction mixture was subjected to the workup procedure outlined in the general procedure and purified by flash chromatography (5\% ethyl acetate/hexanes) to provide the title compound as a white solid (43.2 mg, 45\% yield). ${ }^{1} \mathrm{H}$ NMR (400 MHz, $\left.\mathrm{CDCl}_{3}\right) \delta 7.16$ (s, 1H), 3.00 (hept, $J=$ $6.9 \mathrm{~Hz}, 1 \mathrm{H}), 1.30(\mathrm{~d}, J=6.9 \mathrm{~Hz}, 6 \mathrm{H}) ;{ }^{13} \mathrm{C} \mathrm{NMR}\left(101 \mathrm{MHz}, \mathrm{CDCl}_{3}\right) \delta 180.69,162.44$, 160.39, 117.01, 35.93, 21.39; HRMS (ESI) m/z calculated for $\mathrm{C}_{7} \mathrm{H}_{9} \mathrm{Cl}_{2} \mathrm{~N}_{2}\left[(\mathrm{M}+\mathrm{H})^{+}\right]$ 191.0137, found 191.0137; IR (film) 2922, 2851, 1736, 1550, 1517, 1462, 1376, 1259, $1090,1017,798 \mathrm{~cm}^{-1}$. Spectra data are consistent with those reported in the literature: Org. Biomol. Chem. 2015, 13, 2750-2755.

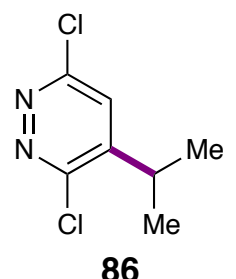

3,6-Dichloro-4-isopropylpyridazine (86): According to the general procedure A, $\mathrm{FeSO}_{4} \cdot 7 \mathrm{H}_{2} \mathrm{O}(7.0 \mathrm{mg}, 0.025 \mathrm{mmol}, 0.05$ equiv.), picolinic acid (6.2 mg, $0.05 \mathrm{mmol}, 0.1$ equiv.), 3,6-dichloropyridazine (76.5 mg, $0.50 \mathrm{mmol}, 1.0$ equiv.), isobutyric acid (445.0 
mg, 5.0 mmol, 10 equiv.), $\mathrm{NaBrO}_{3}$ (150.9 mg, 1.0 mmol, 2.0 equiv.), $4.5 \mathrm{~mL}$ of $\mathrm{H}_{2} \mathrm{O}$ and $0.5 \mathrm{~mL}$ of DMSO, $456 \mathrm{~nm}$ LEDs were used. After 24 hours, the reaction mixture was subjected to the workup procedure outlined in the general procedure and purified by flash chromatography (10\% ethyl acetate/hexanes) to provide the title compound as a white solid (79.7 mg, 82\% yield). ${ }^{1} \mathrm{H}$ NMR (400 MHz, $\left.\mathrm{CDCl}_{3}\right) \delta 7.37$ (s, 1H), 3.24 (hept, $J=$ $6.8 \mathrm{~Hz}, 1 \mathrm{H}), 1.29(\mathrm{~d}, J=6.9 \mathrm{~Hz}, 6 \mathrm{H}) ;{ }^{13} \mathrm{C} \mathrm{NMR}\left(101 \mathrm{MHz}, \mathrm{CDCl}_{3}\right) \delta 156.55,156.25$, 149.80, 126.67, 30.18, 21.14; HRMS (ESI) m/z calculated for $\mathrm{C}_{7} \mathrm{H}_{9} \mathrm{Cl}_{2} \mathrm{~N}_{2}\left[(\mathrm{M}+\mathrm{H})^{+}\right]$ 191.0137, found 191.0137; IR (film) 2964, 1592, 1490, 1406, 1302, 1091, 1043, 910, 863, $762 \mathrm{~cm}^{-1}$. Spectra data are consistent with those reported in the literature: Org. Lett. 2016, $18,3738-3741$.

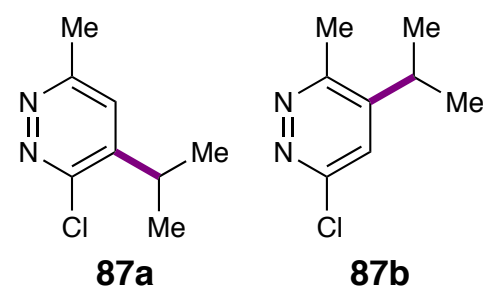

\section{3-Chloro-4-isopropyl-6-methylpyridazine (87a) and 6-chloro-4-isopropyl-3-}

methylpyridazine (87b): According to the general procedure $\mathrm{A}, \mathrm{FeSO}_{4} \cdot 7 \mathrm{H}_{2} \mathrm{O}(7.0 \mathrm{mg}$, $0.025 \mathrm{mmol}, 0.05$ equiv.), picolinic acid (6.2 mg, $0.05 \mathrm{mmol}, 0.1$ equiv.), 3-chloro-6methylpyridazine (65.5 mg, $0.50 \mathrm{mmol}, 1.0$ equiv.), isobutyric acid (445.0 mg, $5.0 \mathrm{mmol}$, 10 equiv.), $\mathrm{NaBrO}_{3}$ (150.9 mg, $1.0 \mathrm{mmol}, 2.0$ equiv.), $4.5 \mathrm{~mL}$ of $\mathrm{H}_{2} \mathrm{O}$ and $0.5 \mathrm{~mL}$ of DMSO, $456 \mathrm{~nm}$ LEDs were used. After 24 hours, the reaction mixture was subjected to the workup procedure outlined in the general procedure and purified by flash chromatography (10\% ethyl acetate/hexanes) to provide the title compounds as a white solid 87a (33.4 mg, 39\% yield) and a white solid $87 \mathbf{b}$ (17.7 mg, 21\% yield). 87a: ${ }^{1} \mathrm{H}$ NMR (400 MHz, $\left.\mathrm{CDCl}_{3}\right) \delta 7.19(\mathrm{~s}, 1 \mathrm{H}), 3.24$ (hept, $\left.J=6.8 \mathrm{~Hz}, 1 \mathrm{H}\right), 2.67$ (s, 3H), 1.27 (d, 
$J=6.9 \mathrm{~Hz}, 6 \mathrm{H}) ;{ }^{13} \mathrm{C} \mathrm{NMR}\left(101 \mathrm{MHz}, \mathrm{CDCl}_{3}\right) \delta 159.77,155.48,146.75,125.66,29.64$, 21.62, 21.35; HRMS (ESI) m/z calculated for $\mathrm{C}_{8} \mathrm{H}_{12} \mathrm{ClN}_{2}\left[(\mathrm{M}+\mathrm{H})^{+}\right] 171.0684$, found 171.0683; IR (film) 2920, 1716, 1543, 1458, 1327, 1298, 1254, 1219, 1165, 1085, 1024, $797 \mathrm{~cm}^{-1}$. Spectra data are consistent with those reported in the literature: J. Heterocyclic Chem. 1991, 28, 583-587. 87b: ${ }^{1} \mathrm{H}$ NMR (400 MHz, $\left.\mathrm{CDCl}_{3}\right) \delta 7.27$ (s, 1H), 3.02 (hept, $J$ $=6.8 \mathrm{~Hz}, 1 \mathrm{H}), 2.69(\mathrm{~s}, 3 \mathrm{H}), 1.25(\mathrm{~d}, J=6.8 \mathrm{~Hz}, 6 \mathrm{H}) ;{ }^{13} \mathrm{C} \mathrm{NMR}\left(101 \mathrm{MHz}, \mathrm{CDCl}_{3}\right) \delta$ $158.44,155.55,149.39,124.08,29.11,21.78,19.40 ;$ HRMS (ESI) $\mathrm{m} / \mathrm{z}$ calculated for $\mathrm{C}_{8} \mathrm{H}_{12} \mathrm{ClN}_{2}\left[(\mathrm{M}+\mathrm{H})^{+}\right]$171.0684, found 171.0683; IR (film) 2976, 2921, 1543, 1468, 1409, $1381,1343,1194,1093,1025,921,795 \mathrm{~cm}^{-1}$. Spectra data are consistent with those reported in the literature: J. Heterocyclic Chem. 1991, 28, 583-587.

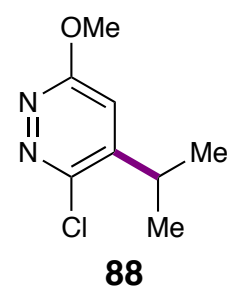

3-Chloro-4-isopropyl-6-methoxypyridazine (88): According to the general procedure A, $\mathrm{FeSO}_{4} \cdot 7 \mathrm{H}_{2} \mathrm{O}(7.0 \mathrm{mg}, 0.025 \mathrm{mmol}, 0.05$ equiv.), picolinic acid $(6.2 \mathrm{mg}, 0.05 \mathrm{mmol}$, 0.1 equiv.), 3-chloro-6-methoxypyridazine ( $74.0 \mathrm{mg}, 0.50 \mathrm{mmol}, 1.0$ equiv.), isobutyric acid (445.0 mg, 5.0 mmol, 10 equiv.), $\mathrm{NaBrO}_{3}(150.9 \mathrm{mg}, 1.0 \mathrm{mmol}, 2.0$ equiv.), $4.5 \mathrm{~mL}$ of $\mathrm{H}_{2} \mathrm{O}$ and $0.5 \mathrm{~mL}$ of DMSO, $456 \mathrm{~nm}$ LEDs were used. After 24 hours, the reaction mixture was subjected to the workup procedure outlined in the general procedure and purified by flash chromatography ( $5 \%$ ethyl acetate/hexanes) to provide the title compound as a colorless oil (63.7 mg, $72 \%$ yield). ${ }^{1} \mathrm{H}$ NMR (400 MHz, $\left.\mathrm{CDCl}_{3}\right) \delta 7.17$ (s, 1H), 4.11 (s, 3H), 3.09 (p, $J=6.9 \mathrm{~Hz}, 1 \mathrm{H}), 1.21$ (d, $J=6.9 \mathrm{~Hz}, 6 \mathrm{H}) ;{ }^{13} \mathrm{C} \mathrm{NMR}(101 \mathrm{MHz}$, $\left.\mathrm{CDCl}_{3}\right) \delta 162.83,151.35,141.03,126.11,55.06,27.08,20.84 ; \mathrm{HRMS}(\mathrm{ESI}) \mathrm{m} / \mathrm{z}$ 
calculated for $\mathrm{C}_{8} \mathrm{H}_{12} \mathrm{ClN}_{2} \mathrm{O}\left[(\mathrm{M}+\mathrm{H})^{+}\right]$187.0633, found 187.0632; IR (film) 2967, 1457, $1375,1336,1216,1085,1008,916,780,747 \mathrm{~cm}^{-1}$.

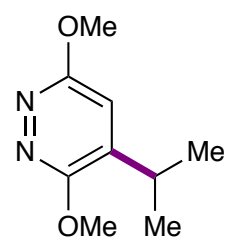

89

4-Isopropyl-3,6-dimethoxypyridazine (89): According to the general procedure A, $\mathrm{FeSO}_{4} \cdot 7 \mathrm{H}_{2} \mathrm{O}$ (7.0 mg, $0.025 \mathrm{mmol}, 0.05$ equiv.), picolinic acid (6.2 mg, $0.05 \mathrm{mmol}, 0.1$ equiv.), 3,6-dimethoxypyridazine (72.0 $\mathrm{mg}, 0.50 \mathrm{mmol}, 1.0$ equiv.), isobutyric acid (445.0 mg, 5.0 mmol, 10 equiv.), $\mathrm{NaBrO}_{3}$ (150.9 mg, $1.0 \mathrm{mmol}, 2.0$ equiv.), $4.5 \mathrm{~mL}$ of $\mathrm{H}_{2} \mathrm{O}$ and $0.5 \mathrm{~mL}$ of DMSO, $456 \mathrm{~nm}$ LEDs were used. After 24 hours, the reaction mixture was subjected to the workup procedure outlined in the general procedure and purified by flash chromatography (10\% ethyl acetate/hexanes) to provide the title compound as a colorless oil (47.0 mg, 51\% yield). ${ }^{1} \mathrm{H}$ NMR (400 $\left.\mathrm{MHz}, \mathrm{CDCl}_{3}\right) \delta 6.72$ (s, 1H), $4.04(\mathrm{~s}, 3 \mathrm{H}), 4.00(\mathrm{~s}, 3 \mathrm{H}), 3.04$ (hept, $J=6.8 \mathrm{~Hz}, 1 \mathrm{H}), 1.18(\mathrm{~d}, J=6.9 \mathrm{~Hz}, 6 \mathrm{H}) ;{ }^{13} \mathrm{C}$ NMR $\left(101 \mathrm{MHz}, \mathrm{CDCl}_{3}\right) \delta 162.53,160.51,141.98,116.19,54.42,54.26,26.99,21.02$ HRMS (ESI) m/z calculated for $\mathrm{C}_{9} \mathrm{H}_{15} \mathrm{~N}_{2} \mathrm{O}_{2}\left[(\mathrm{M}+\mathrm{H})^{+}\right]$183.1128, found 183.1128; IR (film) 2950, 1616, 1461, 1389, 1329, 1231, 1093, 1011, 891, $771 \mathrm{~cm}^{-1}$. Spectra data are consistent with those reported in the literature: Angew. Chem. Int. Ed. 2015, 54, 44784481.<smiles>CCc1nc(C(C)C)cnc1C</smiles>
90 
1-(3-Ethyl-5-isopropylpyrazin-2-yl)ethan-1-one (90): According to the general procedure $\mathrm{A}, \mathrm{FeSO}_{4} \cdot 7 \mathrm{H}_{2} \mathrm{O}(7.0 \mathrm{mg}, 0.025 \mathrm{mmol}, 0.05$ equiv.), picolinic acid (6.2 $\mathrm{mg}$, $0.05 \mathrm{mmol}, 0.1$ equiv.), 1-(3-ethylpyrazin-2-yl)ethanone (76.5 mg, $0.50 \mathrm{mmol}, 1.0$ equiv.), isobutyric acid (445.0 mg, 5.0 mmol, 10 equiv.), $\mathrm{NaBrO}_{3}(150.9 \mathrm{mg}, 1.0 \mathrm{mmol}$, 2.0 equiv.), $4.5 \mathrm{~mL}$ of $\mathrm{H}_{2} \mathrm{O}$ and $0.5 \mathrm{~mL}$ of DMSO, $456 \mathrm{~nm}$ LEDs were used. After 24 hours, the reaction mixture was subjected to the workup procedure outlined in the general procedure and purified by flash chromatography (10\% ethyl acetate/hexanes) to provide the title compound as a white solid (47.3 mg, 49\% yield). ${ }^{1} \mathrm{H} \mathrm{NMR}\left(400 \mathrm{MHz}, \mathrm{CDCl}_{3}\right) \delta$ $8.32(\mathrm{~s}, 1 \mathrm{H}), 3.16-3.08(\mathrm{~m}, 3 \mathrm{H}), 2.67(\mathrm{~s}, 3 \mathrm{H}), 1.33(\mathrm{~d}, J=6.9 \mathrm{~Hz}, 6 \mathrm{H}), 1.26(\mathrm{t}, J=7.4$ $\mathrm{Hz}, 3 \mathrm{H}) ;{ }^{13} \mathrm{C} \mathrm{NMR}\left(101 \mathrm{MHz}, \mathrm{CDCl}_{3}\right) \delta 201.33,163.93,157.85,144.23,138.37,34.24$, 28.88, 28.11, 21.92, 13.16; HRMS (ESI) m/z calculated for $\mathrm{C}_{11} \mathrm{H}_{17} \mathrm{~N}_{2} \mathrm{O}\left[(\mathrm{M}+\mathrm{H})^{+}\right]$ 193.1335, found 193.1335; IR (film) 2956, 2922, 2851, 1721, 1461, 1377, 1259, 1089, $1017,798 \mathrm{~cm}^{-1}$.

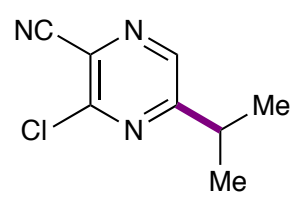

91

3-Chloro-5-isopropylpyrazine-2-carbonitrile (91): According to the general procedure A, $\mathrm{FeSO}_{4} \cdot 7 \mathrm{H}_{2} \mathrm{O}(7.0 \mathrm{mg}, 0.025 \mathrm{mmol}, 0.05$ equiv.), picolinic acid (6.2 mg, $0.05 \mathrm{mmol}$, 0.1 equiv.), 3-chloropyrazine-2-carbonitrile (71.5 $\mathrm{mg}, 0.50 \mathrm{mmol}, 1.0$ equiv.), isobutyric acid (445.0 mg, 5.0 mmol, 10 equiv.), $\mathrm{NaBrO}_{3}(150.9 \mathrm{mg}, 1.0 \mathrm{mmol}, 2.0$ equiv.), $4.0 \mathrm{~mL}$ of $\mathrm{H}_{2} \mathrm{O}$ and $1.0 \mathrm{~mL}$ of DMSO, $440 \mathrm{~nm}$ LEDs were used. After 24 hours, the reaction mixture was subjected to the workup procedure outlined in the general procedure and purified by flash chromatography ( $5 \%$ ethyl acetate/hexanes) to provide the title 
compound as a colorless oil (56.0 mg, $62 \%$ yield). ${ }^{1} \mathrm{H}$ NMR $\left(400 \mathrm{MHz}, \mathrm{CDCl}_{3}\right) \delta 8.50$ (s, 1H), 3.19 (hept, $J=6.9 \mathrm{~Hz}, 1 \mathrm{H}), 1.35(\mathrm{~d}, J=6.9 \mathrm{~Hz}, 6 \mathrm{H}) ;{ }^{13} \mathrm{C} \mathrm{NMR}\left(101 \mathrm{MHz}, \mathrm{CDCl}_{3}\right) \delta$ $166.35,150.80,141.54,127.37,114.03,34.28,21.61 ;$ HRMS (ESI) m/z calculated for $\mathrm{C}_{8} \mathrm{H}_{8} \mathrm{ClN}_{3}\left[\mathrm{M}^{+}\right]$181.0407, found 181.0406; IR (film) 2972, 1549, 1509, 1468, 1338, 1292, $1257,1172,1139,1075,915,864 \mathrm{~cm}^{-1}$.

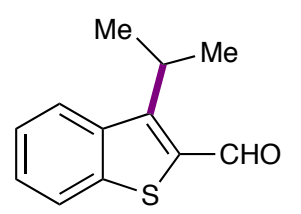

92

3-Isopropylbenzo $[b]$ thiophene-2-carbaldehyde (92): According to the general procedure $\mathrm{A}, \mathrm{FeSO}_{4} \cdot 7 \mathrm{H}_{2} \mathrm{O}(7.0 \mathrm{mg}, 0.025 \mathrm{mmol}, 0.05$ equiv.), picolinic acid $(6.2 \mathrm{mg}$, $0.05 \mathrm{mmol}, 0.1$ equiv.), benzo[b]thiophene-2-carbaldehyde ( $81.0 \mathrm{mg}, 0.50 \mathrm{mmol}, 1.0$ equiv.), isobutyric acid (445.0 mg, $5.0 \mathrm{mmol}, 10$ equiv.), $\mathrm{NaBrO}_{3}(150.9 \mathrm{mg}, 1.0 \mathrm{mmol}$, 2.0 equiv.), $4.0 \mathrm{~mL}$ of $\mathrm{H}_{2} \mathrm{O}$ and $1.0 \mathrm{~mL}$ of DMSO, $440 \mathrm{~nm}$ LEDs were used. After 24 hours, the reaction mixture was subjected to the workup procedure outlined in the general procedure and purified by flash chromatography (5\% ethyl acetate/hexanes) to provide the title compound as a light yellow oil $\left(71.2 \mathrm{mg}, 70 \%\right.$ yield). ${ }^{1} \mathrm{H}$ NMR (400 MHz, $\left.\mathrm{CDCl}_{3}\right) \delta 10.44(\mathrm{~s}, 1 \mathrm{H}), 8.04(\mathrm{~d}, J=8.2 \mathrm{~Hz}, 1 \mathrm{H}), 7.86(\mathrm{~d}, J=8.1 \mathrm{~Hz}, 1 \mathrm{H}), 7.47(\mathrm{t}, J=7.5$ Hz, 1H), 7.40 (t, $J=7.6 \mathrm{~Hz}, 1 \mathrm{H}), 3.99$ (hept, $J=7.1 \mathrm{~Hz}, 1 \mathrm{H}), 1.59$ (d, $J=7.2 \mathrm{~Hz}, 6 \mathrm{H}$ ); ${ }^{13} \mathrm{C}$ NMR $\left(101 \mathrm{MHz}, \mathrm{CDCl}_{3}\right) \delta 184.09,153.13,142.80,138.50,137.51,127.77,125.48$, 124.35, 123.49, 28.29, 23.26; HRMS (ESI) $\mathrm{m} / \mathrm{z}$ calculated for $\mathrm{C}_{12} \mathrm{H}_{13} \mathrm{OS}\left[(\mathrm{M}+\mathrm{H})^{+}\right]$ 205.0682, found 205.0681; IR (film) 2957, 1649, 1568, 1433, 1380, 1254, 1224, 1054, $944,886,831,750 \mathrm{~cm}^{-1}$. 


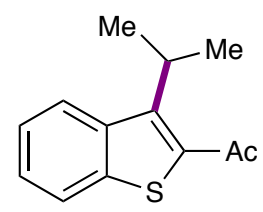

93

1-(3-Isopropylbenzo[b]thiophen-2-yl)ethan-1-one (93): According to the general procedure $\mathrm{B}, \mathrm{FeSO}_{4} \cdot 7 \mathrm{H}_{2} \mathrm{O}(7.0 \mathrm{mg}, 0.025 \mathrm{mmol}, 0.05$ equiv.), picolinic acid (6.2 $\mathrm{mg}$, $0.05 \mathrm{mmol}, 0.1$ equiv.), 1-(benzo[b]thiophen-2-yl)ethenone (90.0 mg, $0.50 \mathrm{mmol}, 1.0$ equiv.), isobutyric acid (445.0 mg, $5.0 \mathrm{mmol}, 10$ equiv.), $\mathrm{NaClO}_{3}(106.5 \mathrm{mg}, 1.0 \mathrm{mmol}$, 2.0 equiv.), $4.0 \mathrm{~mL}$ of $\mathrm{H}_{2} \mathrm{O}$ and $1.0 \mathrm{~mL}$ of DMSO, $427 \mathrm{~nm}$ LEDs were used. After 36 hours, the reaction mixture was subjected to the workup procedure outlined in the general procedure and purified by flash chromatography (5\% ethyl acetate/hexanes) to provide the title compound as a colorless oil $\left(75.2 \mathrm{mg}, 69 \%\right.$ yield). ${ }^{1} \mathrm{H} \mathrm{NMR}\left(400 \mathrm{MHz}, \mathrm{CDCl}_{3}\right) \delta$ $8.14(\mathrm{~d}, J=8.2 \mathrm{~Hz}, 1 \mathrm{H}), 7.84(\mathrm{~d}, J=8.0 \mathrm{~Hz}, 1 \mathrm{H}), 7.45(\mathrm{t}, J=7.3 \mathrm{~Hz}, 1 \mathrm{H}), 7.38(\mathrm{t}, J=7.5$ $\mathrm{Hz}, 1 \mathrm{H}), 4.43$ (hept, $J=7.2 \mathrm{~Hz}, 1 \mathrm{H}), 2.64(\mathrm{~s}, 3 \mathrm{H}), 1.49(\mathrm{~d}, J=7.2 \mathrm{~Hz}, 6 \mathrm{H}) ;{ }^{13} \mathrm{C} \mathrm{NMR}$ $\left(101 \mathrm{MHz}, \mathrm{CDCl}_{3}\right) \delta 193.66,149.36,140.29,138.83,134.38,126.74,125.99,124.12$ 122.99, 31.24, 27.64, 21.36; HRMS (ESI) m/z calculated for $\mathrm{C}_{13} \mathrm{H}_{15} \mathrm{OS}\left[(\mathrm{M}+\mathrm{H})^{+}\right]$ 219.0838, found 219.0839; IR (film) 2960, 1670, 1503, 1422, 1356, 1263, 1211, 1111, $985,950,768,732 \mathrm{~cm}^{-1}$.

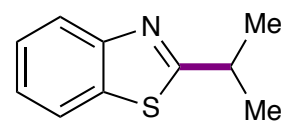

94

2-Isopropylbenzo[d] thiazole (94): According to the general procedure $\mathrm{A}, \mathrm{FeSO}_{4} \cdot 7 \mathrm{H}_{2} \mathrm{O}$ (7.0 mg, $0.025 \mathrm{mmol}, 0.05$ equiv.), picolinic acid (6.2 mg, $0.05 \mathrm{mmol}, 0.1$ equiv.), benzo[d]thiazole $(68.3 \mathrm{mg}, 0.50 \mathrm{mmol}, 1.0$ equiv.), isobutyric acid (445.0 $\mathrm{mg}, 5.0 \mathrm{mmol}$, 10 equiv.), $\mathrm{NaBrO}_{3}$ (150.9 mg, $1.0 \mathrm{mmol}, 2.0$ equiv.), $4.0 \mathrm{~mL}$ of $\mathrm{H}_{2} \mathrm{O}$ and $1.0 \mathrm{~mL}$ of 
DMSO, $440 \mathrm{~nm}$ LEDs were used. After 24 hours, the reaction mixture was subjected to the workup procedure outlined in the general procedure and purified by flash chromatography (5\% ethyl acetate/hexanes) to provide the title compound as a yellow oil (31.8 mg, 36\% yield). ${ }^{1} \mathrm{H}$ NMR (400 MHz, $\left.\mathrm{CDCl}_{3}\right) \delta 7.98(\mathrm{~d}, J=8.2 \mathrm{~Hz}, 1 \mathrm{H}), 7.84(\mathrm{~d}, J$ $=8.0 \mathrm{~Hz}, 1 \mathrm{H}), 7.44(\mathrm{ddd}, J=8.3,7.3,1.3 \mathrm{~Hz}, 1 \mathrm{H}), 7.34(\mathrm{ddd}, J=8.2,7.3,1.2 \mathrm{~Hz}, 1 \mathrm{H})$, 3.43 (hept, $J=6.9 \mathrm{~Hz}, 1 \mathrm{H}), 1.48(\mathrm{~d}, J=6.9 \mathrm{~Hz}, 6 \mathrm{H}) ;{ }^{13} \mathrm{C} \mathrm{NMR}\left(101 \mathrm{MHz}, \mathrm{CDCl}_{3}\right) \delta$ $178.63,153.00,134.60,125.81,124.55,122.50,121.50,34.03,22.86 ;$ HRMS (ESI) m/z calculated for $\mathrm{C}_{10} \mathrm{H}_{12} \mathrm{NS}\left[(\mathrm{M}+\mathrm{H})^{+}\right]$178.0685, found 178.0685; IR (film) 2965, 1517, $1456,1437,1307,1241,1093,1036,999,890,756,728 \mathrm{~cm}^{-1}$. Spectra data are consistent with those reported in the literature: J. Am. Chem. Soc. 2017, 139, 14315-14321.

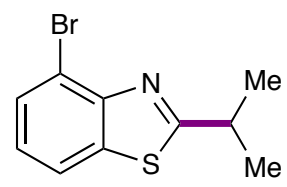

95

4-Bromo-2-isopropylbenzo[d] thiazole (95): According to the general procedure B, $\mathrm{FeSO}_{4} \cdot 7 \mathrm{H}_{2} \mathrm{O}$ (7.0 mg, $0.025 \mathrm{mmol}, 0.05$ equiv.), picolinic acid $(6.2 \mathrm{mg}, 0.05 \mathrm{mmol}, 0.1$ equiv.), 4-bromobenzo[d]thiazole ( $107.0 \mathrm{mg}, 0.50 \mathrm{mmol}, 1.0$ equiv.), isobutyric acid (445.0 mg, 5.0 mmol, 10 equiv.), $\mathrm{NaClO}_{3}(106.5 \mathrm{mg}, 1.0 \mathrm{mmol}, 2.0$ equiv.), $4.0 \mathrm{~mL}$ of $\mathrm{H}_{2} \mathrm{O}$ and $1.0 \mathrm{~mL}$ of DMSO, $427 \mathrm{~nm}$ LEDs were used. After 36 hours, the reaction mixture was subjected to the workup procedure outlined in the general procedure and purified by flash chromatography ( $5 \%$ ethyl acetate/hexanes) to provide the title compound as a colorless oil (55.3 mg, 43\% yield). ${ }^{1} \mathrm{H}$ NMR (400 MHz, $\left.\mathrm{CDCl}_{3}\right) \delta 7.79$ (d, $J=8.0 \mathrm{~Hz}, 1 \mathrm{H}), 7.64(\mathrm{~d}, J=7.8 \mathrm{~Hz}, 1 \mathrm{H}), 7.19$ (t, $J=7.9 \mathrm{~Hz}, 1 \mathrm{H}), 3.53$ (hept, $J=6.9 \mathrm{~Hz}$, 1H), $1.48(\mathrm{~d}, J=6.9 \mathrm{~Hz}, 6 \mathrm{H}) ;{ }^{13} \mathrm{C} \mathrm{NMR}\left(101 \mathrm{MHz}, \mathrm{CDCl}_{3}\right) \delta 179.85,151.24,135.60$, 
129.29, 125.41, 120.76, 116.05, 34.37, 23.17; HRMS (ESI) m/z calculated for

$\mathrm{C}_{10} \mathrm{H}_{11} \mathrm{BrNS}\left[(\mathrm{M}+\mathrm{H})^{+}\right]$255.9790, found 255.9790; IR (film) 2965, 1510, 1450, 1400, $1306,1197,1093,1002,887,765 \mathrm{~cm}^{-1}$.

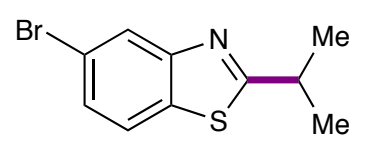

96

5-Bromo-2-isopropylbenzo $[d]$ thiazole (96): According to the general procedure B, $\mathrm{FeSO}_{4} \cdot 7 \mathrm{H}_{2} \mathrm{O}(7.0 \mathrm{mg}, 0.025 \mathrm{mmol}, 0.05$ equiv.), picolinic acid $(6.2 \mathrm{mg}, 0.05 \mathrm{mmol}, 0.1$ equiv.), 5-bromobenzo[d]thiazole ( $107.0 \mathrm{mg}, 0.50 \mathrm{mmol}, 1.0$ equiv.), isobutyric acid (445.0 mg, 5.0 mmol, 10 equiv.), $\mathrm{NaClO}_{3}(106.5 \mathrm{mg}, 1.0 \mathrm{mmol}, 2.0$ equiv.), $4.0 \mathrm{~mL}$ of $\mathrm{H}_{2} \mathrm{O}$ and $1.0 \mathrm{~mL}$ of DMSO, $427 \mathrm{~nm}$ LEDs were used. After 36 hours, the reaction mixture was subjected to the workup procedure outlined in the general procedure and purified by flash chromatography ( $5 \%$ ethyl acetate/hexanes) to provide the title compound as a colorless oil (47.0 mg, 37\% yield). ${ }^{1} \mathrm{H}$ NMR (400 MHz, $\left.\mathrm{CDCl}_{3}\right) \delta 8.12$ (d, $J=1.8 \mathrm{~Hz}, 1 \mathrm{H}), 7.69(\mathrm{~d}, J=8.5 \mathrm{~Hz}, 1 \mathrm{H}), 7.45(\mathrm{dd}, J=8.5,1.9 \mathrm{~Hz}, 1 \mathrm{H}), 3.41$ (hept, $J=$ $6.9 \mathrm{~Hz}, 1 \mathrm{H}), 1.47(\mathrm{~d}, J=6.9 \mathrm{~Hz}, 6 \mathrm{H}) ;{ }^{13} \mathrm{C} \mathrm{NMR}\left(101 \mathrm{MHz}, \mathrm{CDCl}_{3}\right) \delta 180.48,154.27$, 133.47, 127.68, 125.50, 122.57, 119.40, 34.11, 22.79; HRMS (ESI) m/z calculated for $\mathrm{C}_{10} \mathrm{H}_{11} \mathrm{BrNS}\left[(\mathrm{M}+\mathrm{H})^{+}\right]$255.9790, found 255.9791; IR (film) 2964, 1510, 1461, 1431, $1247,1146,1030,903,863,794,725 \mathrm{~cm}^{-1}$.

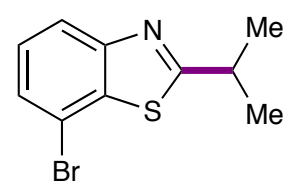

97

7-Bromo-2-isopropylbenzo[d] thiazole (97): According to the general procedure B, $\mathrm{FeSO}_{4} \cdot 7 \mathrm{H}_{2} \mathrm{O}$ ( $7.0 \mathrm{mg}, 0.025 \mathrm{mmol}, 0.05$ equiv.), picolinic acid $(6.2 \mathrm{mg}, 0.05 \mathrm{mmol}, 0.1$ 
equiv.), 7-bromobenzo[d]thiazole (107.0 $\mathrm{mg}, 0.50 \mathrm{mmol}, 1.0$ equiv.), isobutyric acid (445.0 mg, 5.0 mmol, 10 equiv.), $\mathrm{NaClO}_{3}(106.5 \mathrm{mg}, 1.0 \mathrm{mmol}, 2.0$ equiv.), $4.0 \mathrm{~mL}$ of $\mathrm{H}_{2} \mathrm{O}$ and $1.0 \mathrm{~mL}$ of DMSO, $427 \mathrm{~nm}$ LEDs were used. After 36 hours, the reaction mixture was subjected to the workup procedure outlined in the general procedure and purified by flash chromatography ( $5 \%$ ethyl acetate/hexanes) to provide the title compound as a colorless oil (84.9 mg, 66\% yield). ${ }^{1} \mathrm{H}$ NMR (400 MHz, $\left.\mathrm{CDCl}_{3}\right) \delta 7.90$ (d, $J=8.1 \mathrm{~Hz}, 1 \mathrm{H}), 7.46(\mathrm{~d}, J=7.8 \mathrm{~Hz}, 1 \mathrm{H}), 7.31(\mathrm{t}, J=7.9 \mathrm{~Hz}, 1 \mathrm{H}), 3.41$ (hept, $J=6.9 \mathrm{~Hz}$, $1 \mathrm{H}), 1.48(\mathrm{~d}, J=6.9 \mathrm{~Hz}, 6 \mathrm{H}) ;{ }^{13} \mathrm{C} \mathrm{NMR}\left(101 \mathrm{MHz}, \mathrm{CDCl}_{3}\right) \delta 178.87,152.89,137.53$, 127.36, 126.99, 121.36, 113.91, 34.17, 22.79; HRMS (ESI) m/z calculated for $\mathrm{C}_{10} \mathrm{H}_{11} \mathrm{BrNS}\left[(\mathrm{M}+\mathrm{H})^{+}\right]$255.9790, found 255.9790; IR (film) 2965, 1544, 1514, 1448, $1395,1307,1092,1027,917,779,717 \mathrm{~cm}^{-1}$.

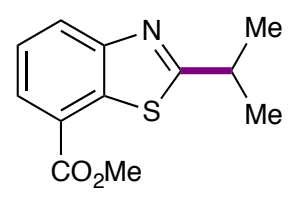

98

Methyl 2-isopropylbenzo[d] thiazole-7-carboxylate (98): According to the general procedure $\mathrm{A}, \mathrm{FeSO}_{4} \cdot 7 \mathrm{H}_{2} \mathrm{O}(7.0 \mathrm{mg}, 0.025 \mathrm{mmol}, 0.05$ equiv.), picolinic acid $(6.2 \mathrm{mg}$, $0.05 \mathrm{mmol}, 0.1$ equiv.), methyl benzo[ $d]$ thiazole-7-carboxylate $(101.7 \mathrm{mg}, 0.50 \mathrm{mmol}$, 1.0 equiv.), isobutyric acid (445.0 mg, $5.0 \mathrm{mmol}, 10$ equiv.), $\mathrm{NaBrO}_{3}(150.9 \mathrm{mg}, 1.0$ mmol, 2.0 equiv.), $4.0 \mathrm{~mL}$ of $\mathrm{H}_{2} \mathrm{O}$ and $1.0 \mathrm{~mL}$ of DMSO, $440 \mathrm{~nm}$ LEDs were used. After 24 hours, the reaction mixture was subjected to the workup procedure outlined in the general procedure and purified by flash chromatography (5\% ethyl acetate/hexanes) to provide the title compound as white solid (39.3 mg, 33\% yield). ${ }^{1} \mathrm{H}$ NMR (400 MHz, $\left.\mathrm{CDCl}_{3}\right) \delta 8.16(\mathrm{dd}, J=8.0,1.1 \mathrm{~Hz}, 1 \mathrm{H}), 8.07(\mathrm{dd}, J=7.6,1.1 \mathrm{~Hz}, 1 \mathrm{H}), 7.51(\mathrm{t}, J=7.8 \mathrm{~Hz}$, 
1H), 4.00 (s, 3H), 3.45 (hept, $J=6.9 \mathrm{~Hz}, 1 \mathrm{H}), 1.50$ (d, $J=6.9 \mathrm{~Hz}, 6 \mathrm{H}) ;{ }^{13} \mathrm{C}$ NMR $(101$ $\left.\mathrm{MHz}, \mathrm{CDCl}_{3}\right) \delta 181.72,166.24,154.04,135.37,127.13,126.48,125.57,124.00,52.50$, 33.85, 22.79; HRMS (ESI) m/z calculated for $\mathrm{C}_{12} \mathrm{H}_{14} \mathrm{NO}_{2} \mathrm{~S}\left[(\mathrm{M}+\mathrm{H})^{+}\right] 236.0740$, found 236.0739; IR (film) 2963, 1706, 1511, 1436, 1402, 1287, 1261, 1035, 919, 813, $749 \mathrm{~cm}^{-1}$.

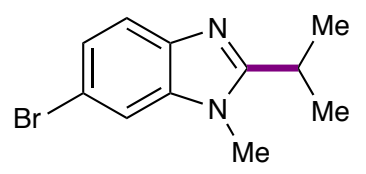

99

6-Bromo-2-isopropyl-1-methyl-1H-benzo[d]imidazole (99): According to the general procedure $\mathrm{A}, \mathrm{FeSO}_{4} \cdot 7 \mathrm{H}_{2} \mathrm{O}(7.0 \mathrm{mg}, 0.025 \mathrm{mmol}, 0.05$ equiv.), picolinic acid (6.2 $\mathrm{mg}$, $0.05 \mathrm{mmol}, 0.1$ equiv.), 6-bromo-1-methyl-1H-benzo[d]imidazole (111.0 mg, $0.50 \mathrm{mmol}$, 1.0 equiv.), isobutyric acid (445.0 mg, $5.0 \mathrm{mmol}, 10$ equiv.), $\mathrm{NaBrO}_{3}(150.9 \mathrm{mg}, 1.0$ mmol, 2.0 equiv.), $4.5 \mathrm{~mL}$ of $\mathrm{H}_{2} \mathrm{O}$ and $0.5 \mathrm{~mL}$ of DMSO, $456 \mathrm{~nm}$ LEDs were used. After 24 hours, the reaction mixture was subjected to the workup procedure outlined in the general procedure and purified by flash chromatography (5\% ethyl acetate/hexanes) to provide the title compound as a white solid (63.4 mg, 50\% yield). ${ }^{1} \mathrm{H}$ NMR (400 MHz, $\left.\mathrm{CDCl}_{3}\right) \delta 7.58(\mathrm{~d}, J=8.5 \mathrm{~Hz}, 1 \mathrm{H}), 7.42(\mathrm{~d}, J=1.6 \mathrm{~Hz}, 1 \mathrm{H}), 7.31(\mathrm{dd}, J=8.5,1.7 \mathrm{~Hz}, 1 \mathrm{H})$, 3.69 (s, 3H), 3.18 (hept, $J=6.9 \mathrm{~Hz}, 1 \mathrm{H}), 1.42(\mathrm{~d}, J=6.9 \mathrm{~Hz}, 6 \mathrm{H}) ;{ }^{13} \mathrm{C}$ NMR $(101 \mathrm{MHz}$, $\left.\mathrm{CDCl}_{3}\right) \delta 160.48,141.07,136.75,124.98,120.35,115.12,112.02,29.66,26.45,20.99 ;$ HRMS (ESI) $\mathrm{m} / \mathrm{z}$ calculated for $\mathrm{C}_{11} \mathrm{H}_{14} \mathrm{BrN}_{2}\left[(\mathrm{M}+\mathrm{H})^{+}\right]$253.0335, found 253.0334; IR (film) 2964, 1611, 1514, 1470, 1401, 1273, 1229, 1084, 917, 833, $816 \mathrm{~cm}^{-1}$.<smiles>CCOC(=O)c1cccc2c1nc(C(C)C)n2C(C)C</smiles> 


\section{Ethyl 2-isopropyl-1-methyl-1 $H$-benzo $[d]$ imidazole-4-carboxylate (100a) and ethyl}

2,5-diisopropyl-1-methyl-1H-benzo $[d]$ imidazole-4-carboxylate (100b): According to the general procedure $\mathrm{A}, \mathrm{FeSO}_{4} \cdot 7 \mathrm{H}_{2} \mathrm{O}(7.0 \mathrm{mg}, 0.025 \mathrm{mmol}, 0.05$ equiv. $)$, picolinic acid (6.2 mg, $0.05 \mathrm{mmol}, 0.1$ equiv.), ethyl 1-methyl-1H-benzo[ $d]$ imidazole-4-carboxylate (107.5 mg, $0.50 \mathrm{mmol}, 1.0$ equiv.), isobutyric acid (445.0 $\mathrm{mg}, 5.0 \mathrm{mmol}, 10$ equiv.), $\mathrm{NaBrO}_{3}$ (150.9 mg, $1.0 \mathrm{mmol}, 2.0$ equiv.), $4.0 \mathrm{~mL}$ of $\mathrm{H}_{2} \mathrm{O}$ and $1.0 \mathrm{~mL}$ of DMSO, $440 \mathrm{~nm}$ LEDs were used. After 24 hours, the reaction mixture was subjected to the workup procedure outlined in the general procedure and purified by flash chromatography $(50 \%$ ethyl acetate/hexanes) to provide the title compounds as a white solid 100a $(90.8 \mathrm{mg}$, 74\% yield) and a white solid 100b (22.4 mg, 16\% yield). 100a: ${ }^{1} \mathrm{H}$ NMR (400 MHz, $\left.\mathrm{CDCl}_{3}\right) \delta 7.89(\mathrm{dd}, J=7.6,1.1 \mathrm{~Hz}, 1 \mathrm{H}), 7.44(\mathrm{dd}, J=8.0,1.1 \mathrm{~Hz}, 1 \mathrm{H}), 7.24(\mathrm{t}, J=7.8 \mathrm{~Hz}$, 1H), $4.46(\mathrm{q}, J=7.1 \mathrm{~Hz}, 2 \mathrm{H}), 3.75(\mathrm{~s}, 3 \mathrm{H}), 3.26$ (hept, $J=6.9 \mathrm{~Hz}, 1 \mathrm{H}), 1.47$ (d, $J=6.9$ $\mathrm{Hz}, 6 \mathrm{H}), 1.44(\mathrm{t}, J=7.2 \mathrm{~Hz}, 3 \mathrm{H}) ;{ }^{13} \mathrm{C} \mathrm{NMR}\left(101 \mathrm{MHz}, \mathrm{CDCl}_{3}\right) \delta 166.15,161.37,141.66$, $137.12,124.38,120.99,120.92,113.09,60.69,29.74,26.90,20.74,14.24 ;$ HRMS (ESI) $\mathrm{m} / \mathrm{z}$ calculated for $\mathrm{C}_{14} \mathrm{H}_{19} \mathrm{BrN}_{2} \mathrm{O}_{2}\left[(\mathrm{M}+\mathrm{H})^{+}\right]$247.1441, found 247.1440; IR (film) 2973, 1700, 1610, 1461, 1423, 1275, 1245, 1199, 1088, 1027, $750 \mathrm{~cm}^{-1}$. 100b: ${ }^{1} \mathrm{H}$ NMR (400 $\left.\mathrm{MHz} \mathrm{CDCl}_{3}\right) \delta 7.84(\mathrm{~d}, J=8.0 \mathrm{~Hz}, 1 \mathrm{H}), 7.16(\mathrm{~d}, J=8.1 \mathrm{~Hz}, 1 \mathrm{H}), 4.45(\mathrm{q}, J=7.1 \mathrm{~Hz}$ 2H), 3.99 (s, 3H), 3.78 (hept, $J=6.8 \mathrm{~Hz}, 1 \mathrm{H}), 3.27$ (hept, $J=6.7 \mathrm{~Hz}, 1 \mathrm{H}), 1.49$ (d, $J=6.9$ $\mathrm{Hz}, 6 \mathrm{H}), 1.43(\mathrm{t}, J=7.1 \mathrm{~Hz}, 3 \mathrm{H}), 1.37(\mathrm{~d}, J=6.8 \mathrm{~Hz}, 6 \mathrm{H}) ;{ }^{13} \mathrm{C} \mathrm{NMR}\left(101 \mathrm{MHz}, \mathrm{CDCl}_{3}\right)$ $\delta 166.37,161.63,142.34,137.51,134.16,124.85,118.80,118.33,60.56,32.67,27.65$, 26.85, 24.08, 20.93, 14.34; HRMS (ESI) m/z calculated for $\mathrm{C}_{17} \mathrm{H}_{25} \mathrm{~N}_{2} \mathrm{O}_{2}\left[(\mathrm{M}+\mathrm{H})^{+}\right]$ 289.1911, found 289.1909; IR (film) 2960, 1717, 1596, 1457, 1252, 1219, 1165, 1086, $1037,797,751 \mathrm{~cm}^{-1}$. 


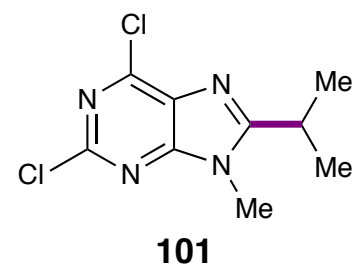

2,6-Dichloro-8-isopropyl-9-methyl-9H-purine (101): According to the general procedure $\mathrm{B}, \mathrm{FeSO}_{4} \cdot 7 \mathrm{H}_{2} \mathrm{O}(7.0 \mathrm{mg}, 0.025 \mathrm{mmol}, 0.05$ equiv.), picolinic acid $(6.2 \mathrm{mg}$, 0.05 mmol, 0.1 equiv.), 2,6-dichloro-9-methyl-9H-purine (104.5 mg, $0.50 \mathrm{mmol}, 1.0$ equiv.), isobutyric acid (445.0 mg, $5.0 \mathrm{mmol}, 10$ equiv.), $\mathrm{NaClO}_{3}(106.5 \mathrm{mg}, 1.0 \mathrm{mmol}$, 2.0 equiv.), $4.0 \mathrm{~mL}$ of $\mathrm{H}_{2} \mathrm{O}$ and $1.0 \mathrm{~mL}$ of DMSO, $427 \mathrm{~nm}$ LEDs were used. After 36 hours, the reaction mixture was subjected to the workup procedure outlined in the general procedure and purified by flash chromatography (10\% ethyl acetate/hexanes) to provide the title compound as a white solid $\left(97.8 \mathrm{mg}, 80 \%\right.$ yield). ${ }^{1} \mathrm{H} \mathrm{NMR}\left(400 \mathrm{MHz}, \mathrm{CDCl}_{3}\right) \delta$ $3.80(\mathrm{~s}, 3 \mathrm{H}), 3.24$ (hept, $J=6.7 \mathrm{~Hz}, 1 \mathrm{H}), 1.45(\mathrm{~d}, J=6.8 \mathrm{~Hz}, 6 \mathrm{H}) ;{ }^{13} \mathrm{C}$ NMR $(101 \mathrm{MHz}$, $\left.\mathrm{CDCl}_{3}\right) \delta 163.93,154.74,151.63,149.47,129.99,29.21,27.25,20.51 ; \mathrm{HRMS}(\mathrm{ESI}) \mathrm{m} / \mathrm{z}$ calculated for $\mathrm{C}_{9} \mathrm{H}_{11} \mathrm{Cl}_{2} \mathrm{~N}_{4}\left[(\mathrm{M}+\mathrm{H})^{+}\right]$245.0355, found 245.0355; IR (film) 2926, 1558, $1482,1356,1285,1252,1151,1081,974,876,789 \mathrm{~cm}^{-1}$. 


\section{NMR Spectra}

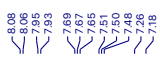

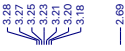

影

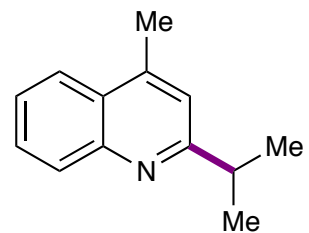

13

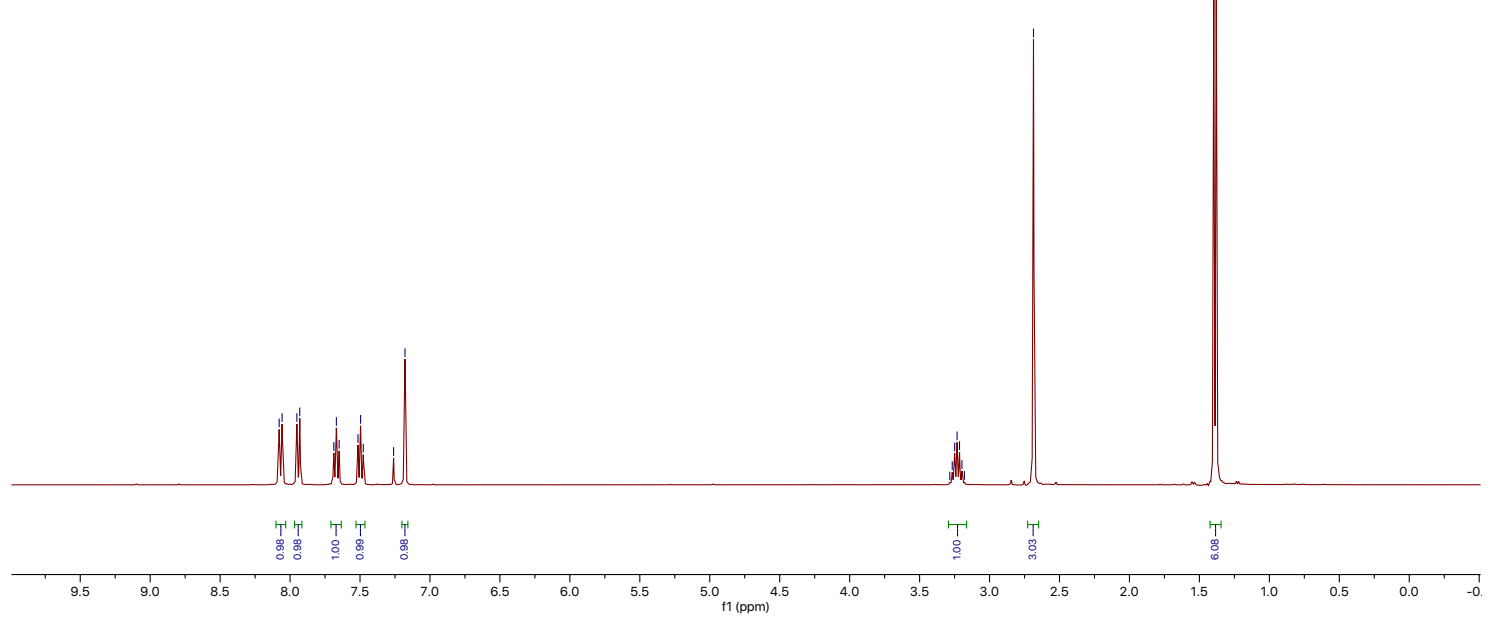

$|z-4-3 A-P|$

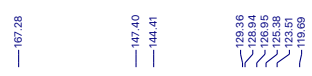
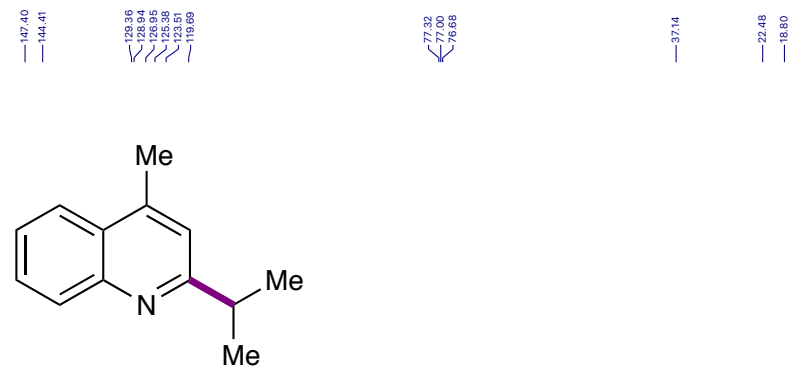

13

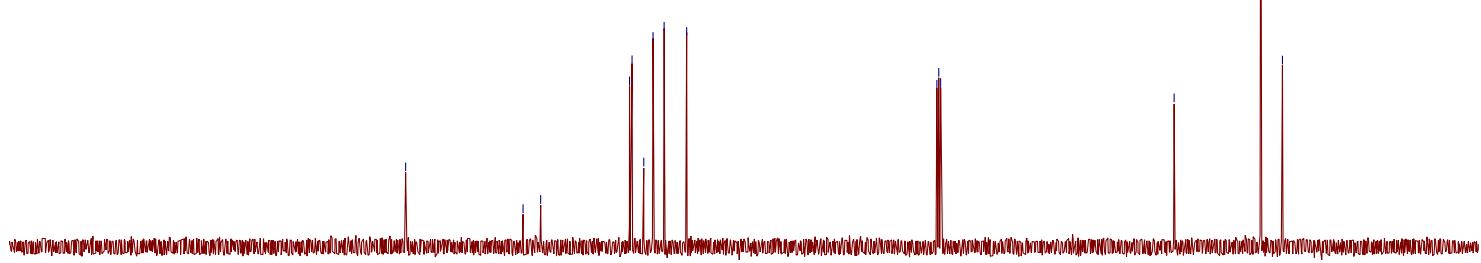

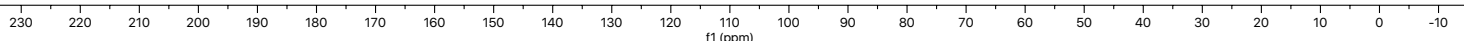




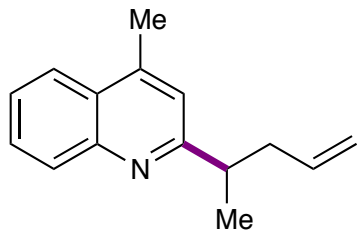

14

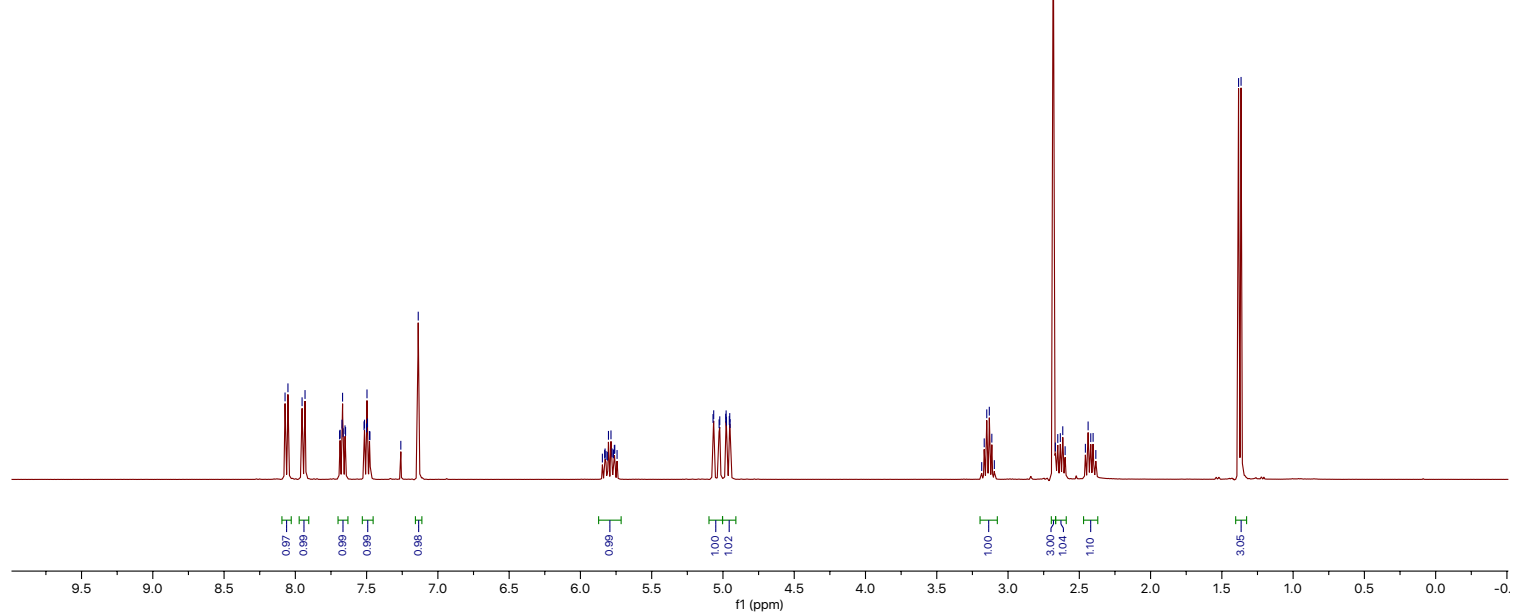

|z-4-11B-P

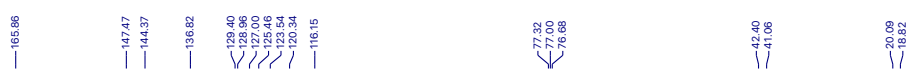<smiles>[M]C(CC=C)c1cc(C)c2ccccc2n1</smiles>

14

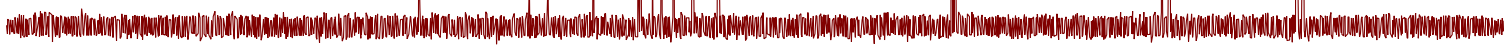

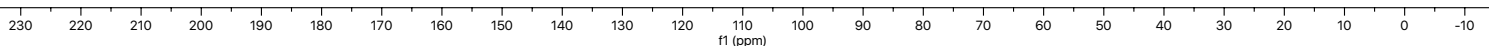



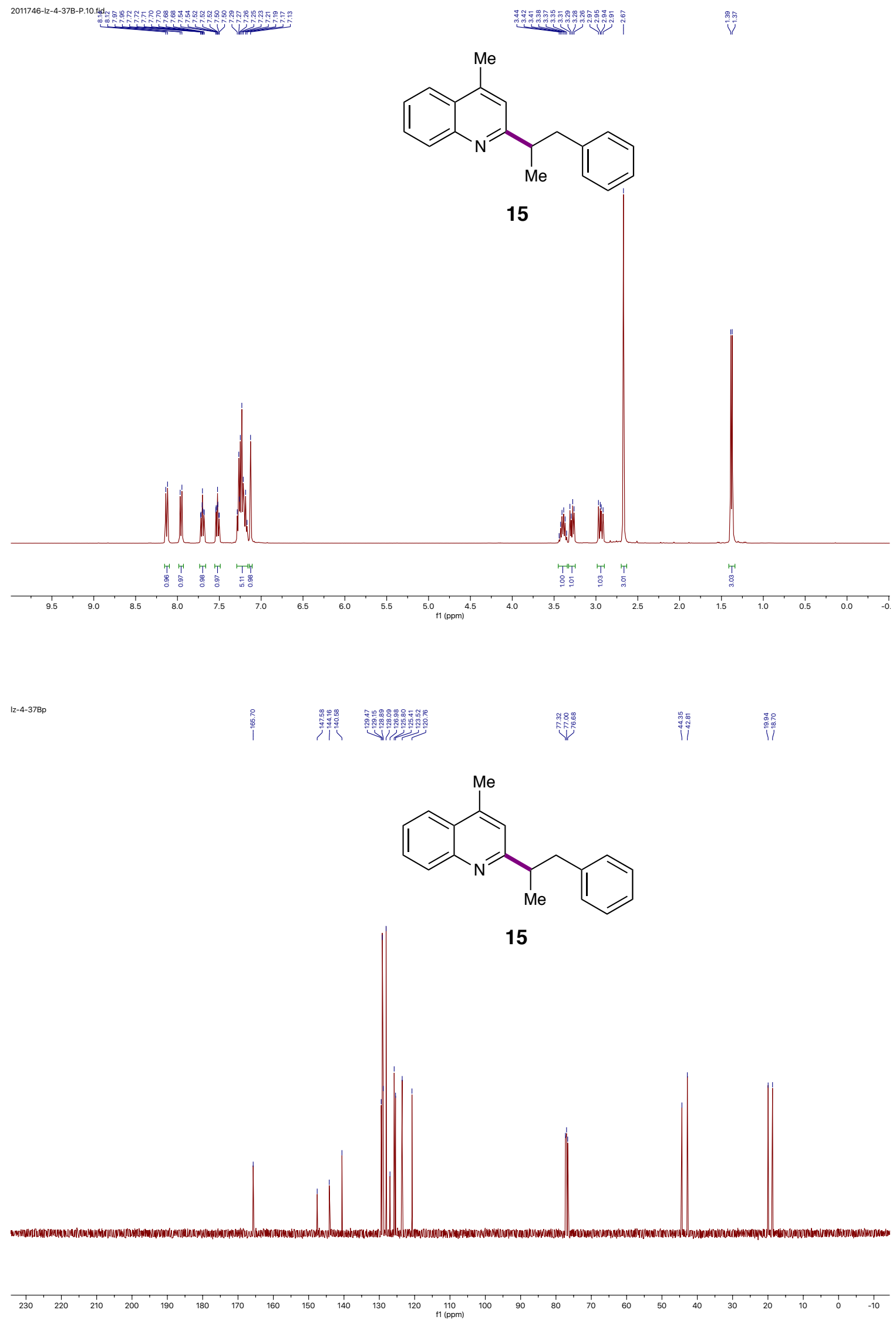


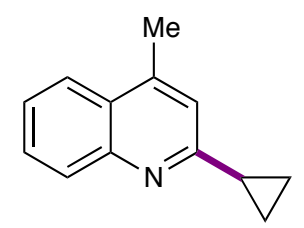

16

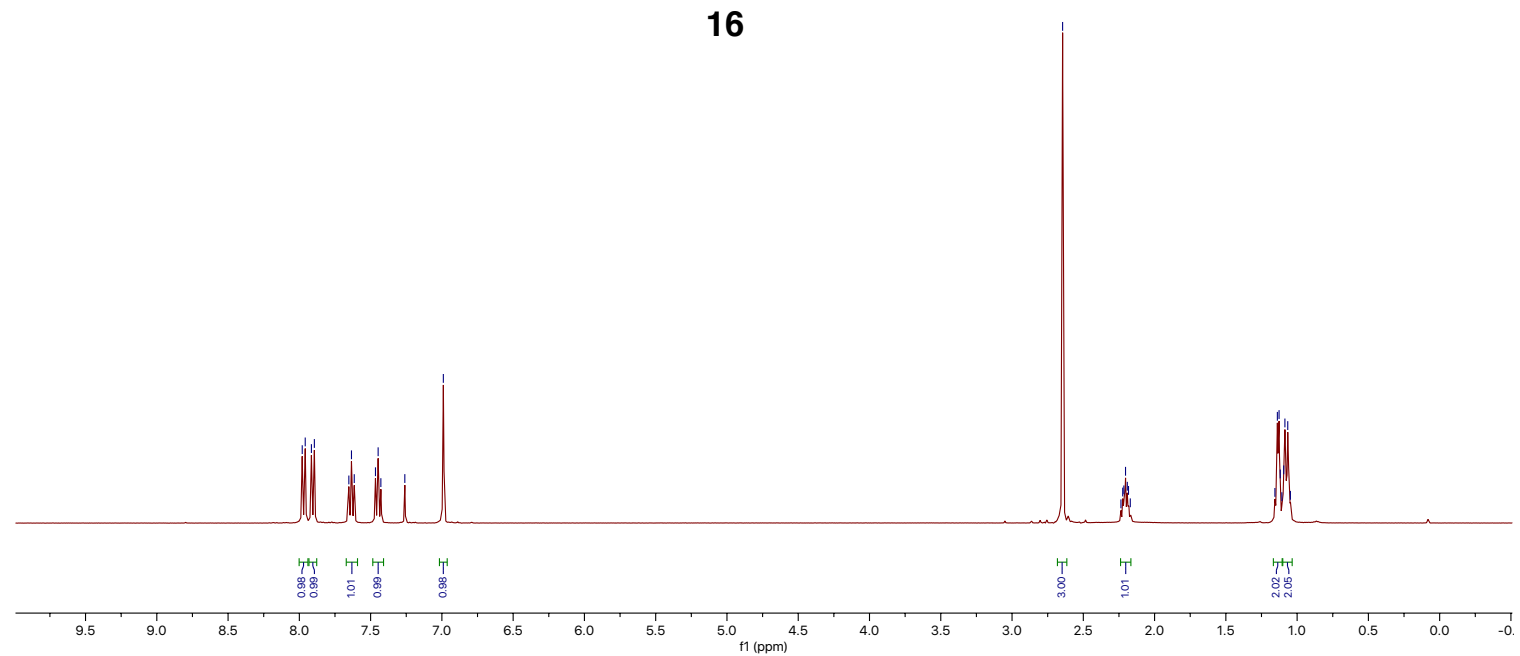

|z-4-14-P

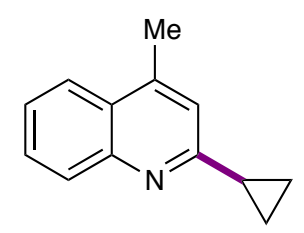

16
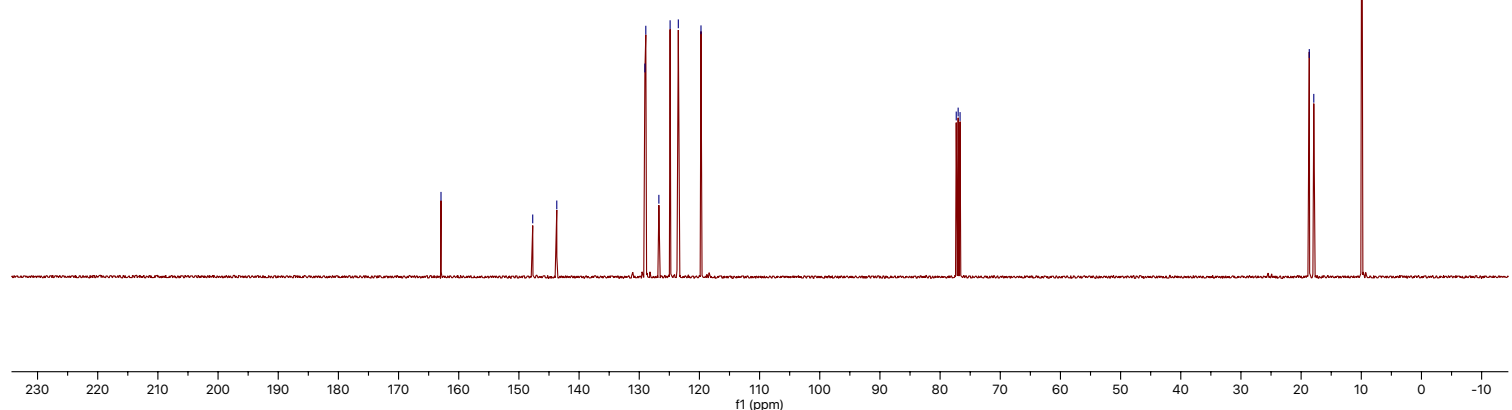


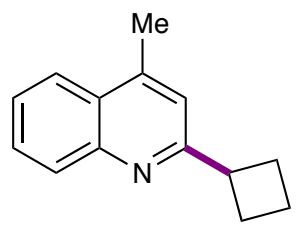

17

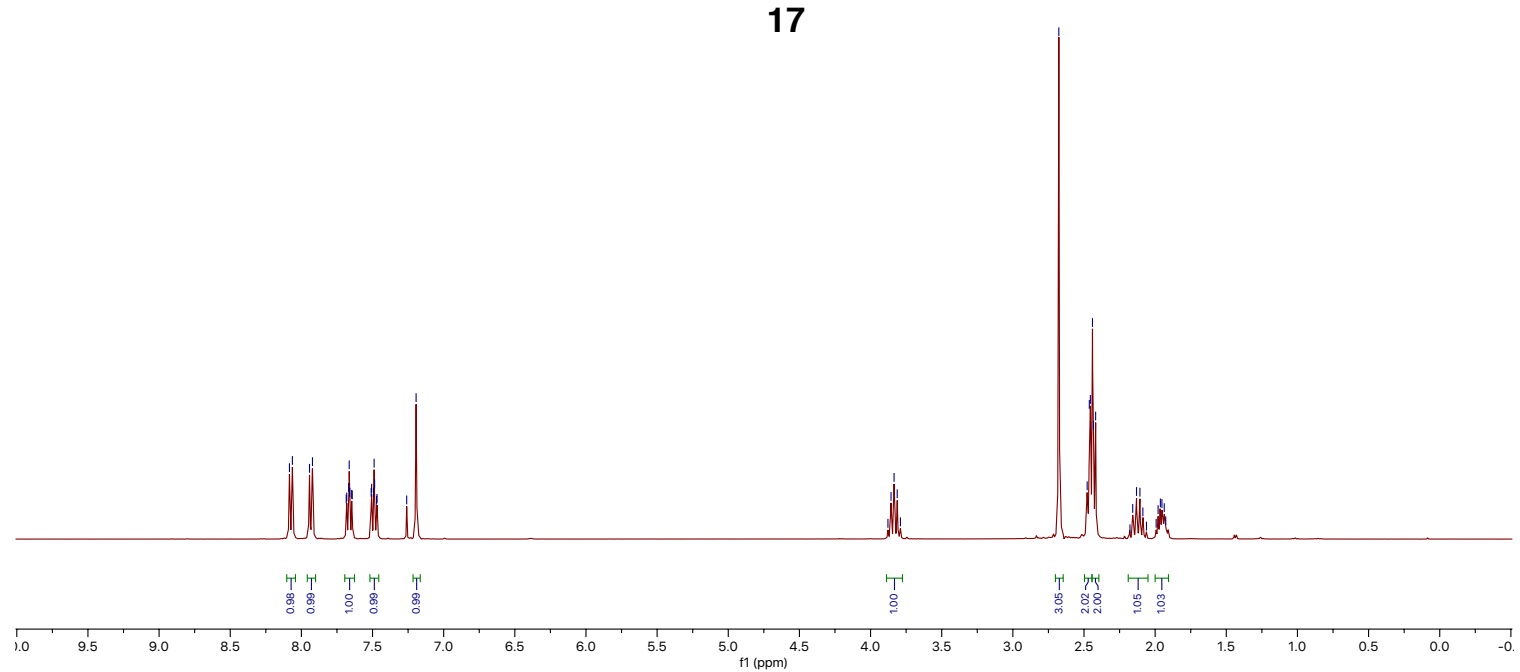

|z-4-18B-p1

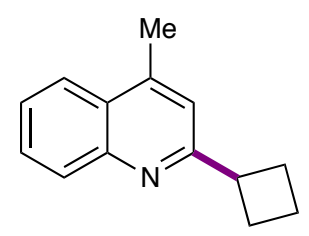

17

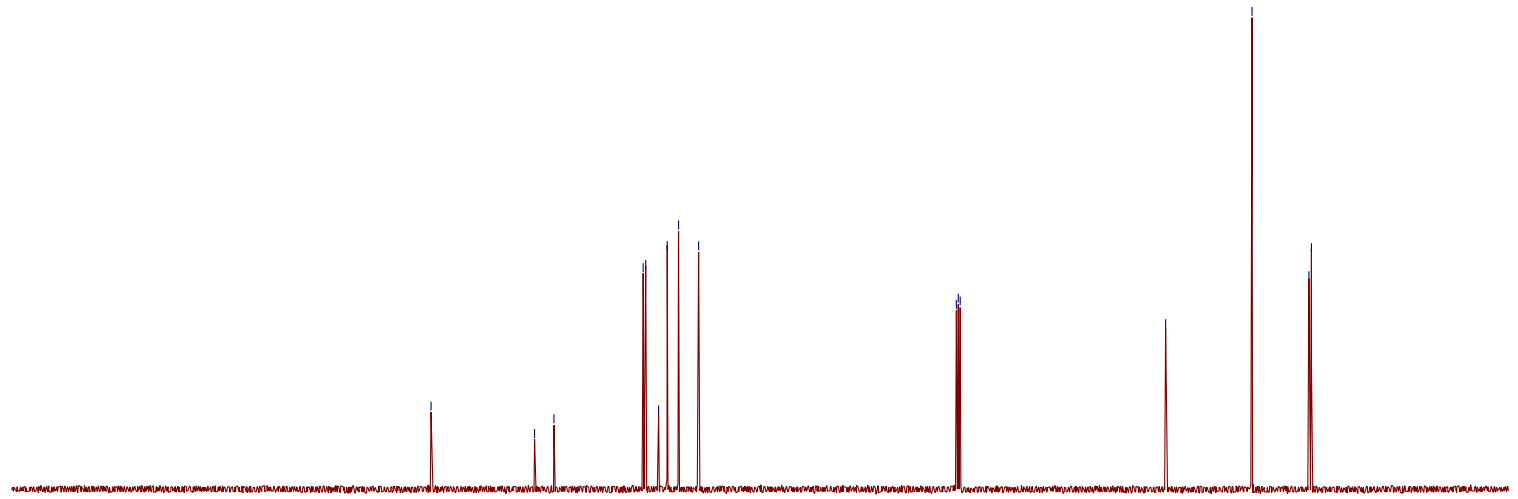

230

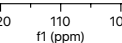




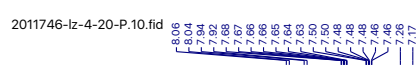

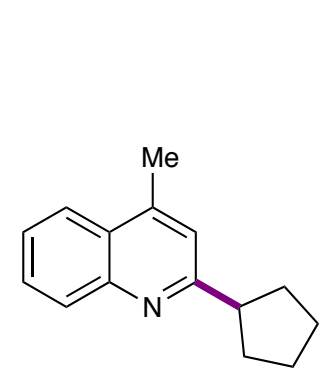

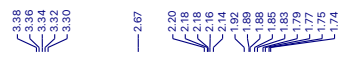

18

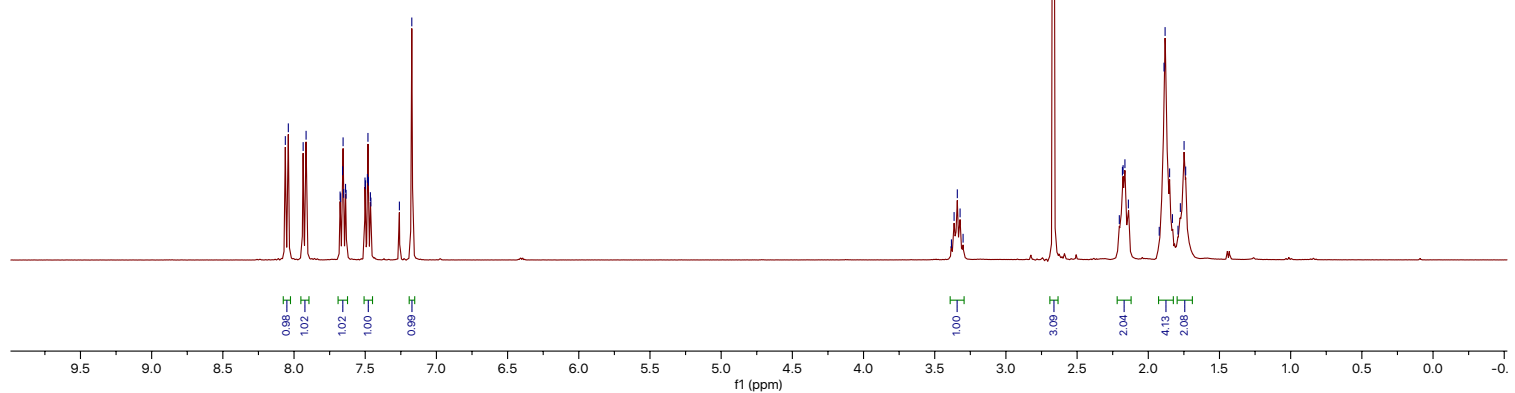

1z-4-20p

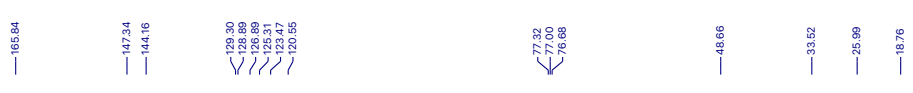

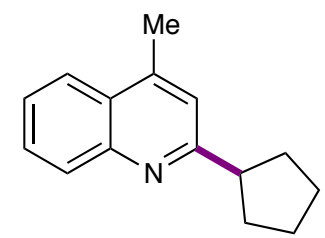

18

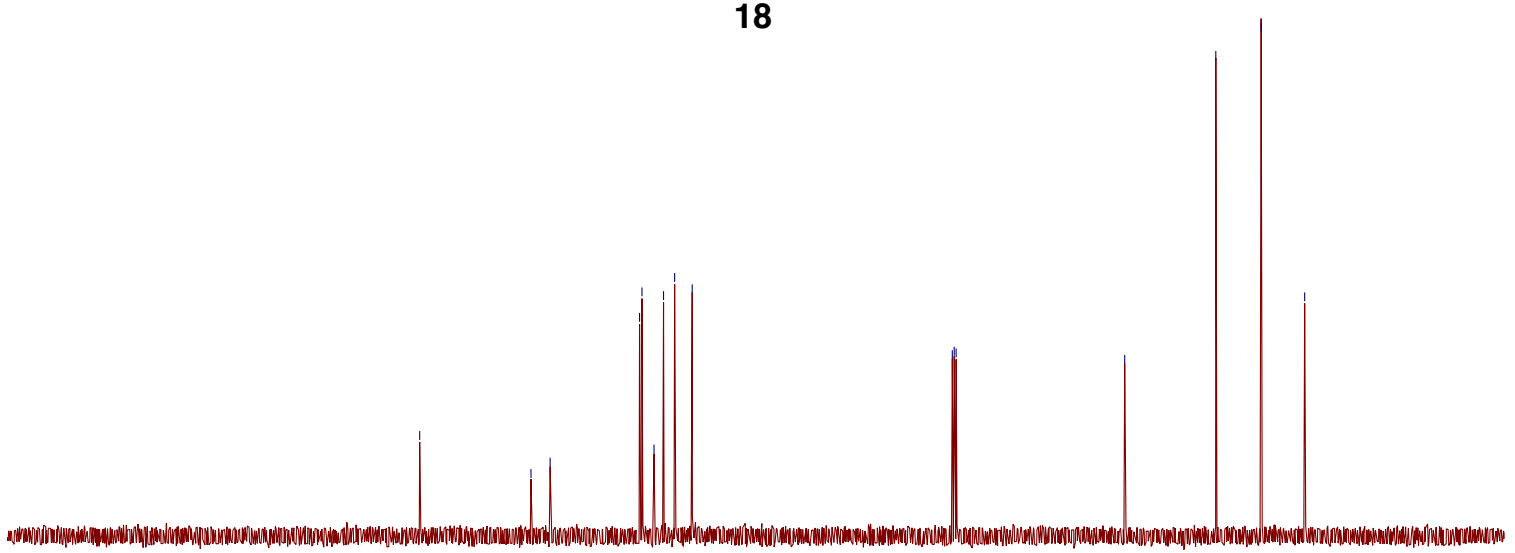

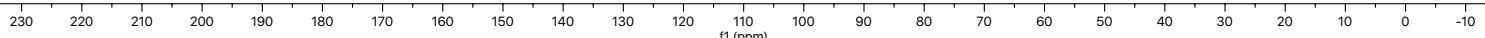




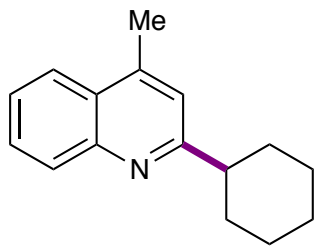

19

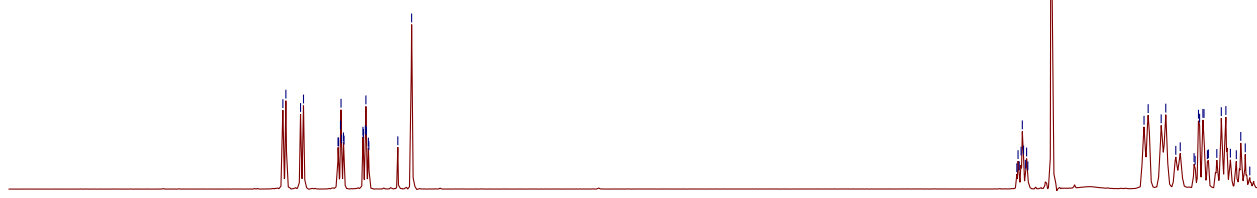

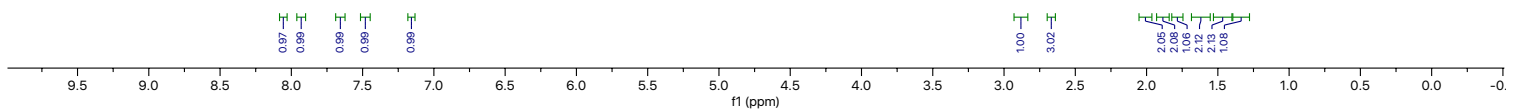

|z-4-23B-P1

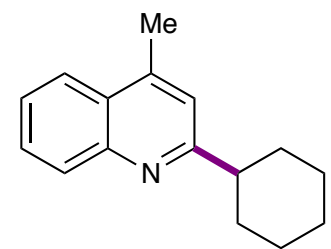

19

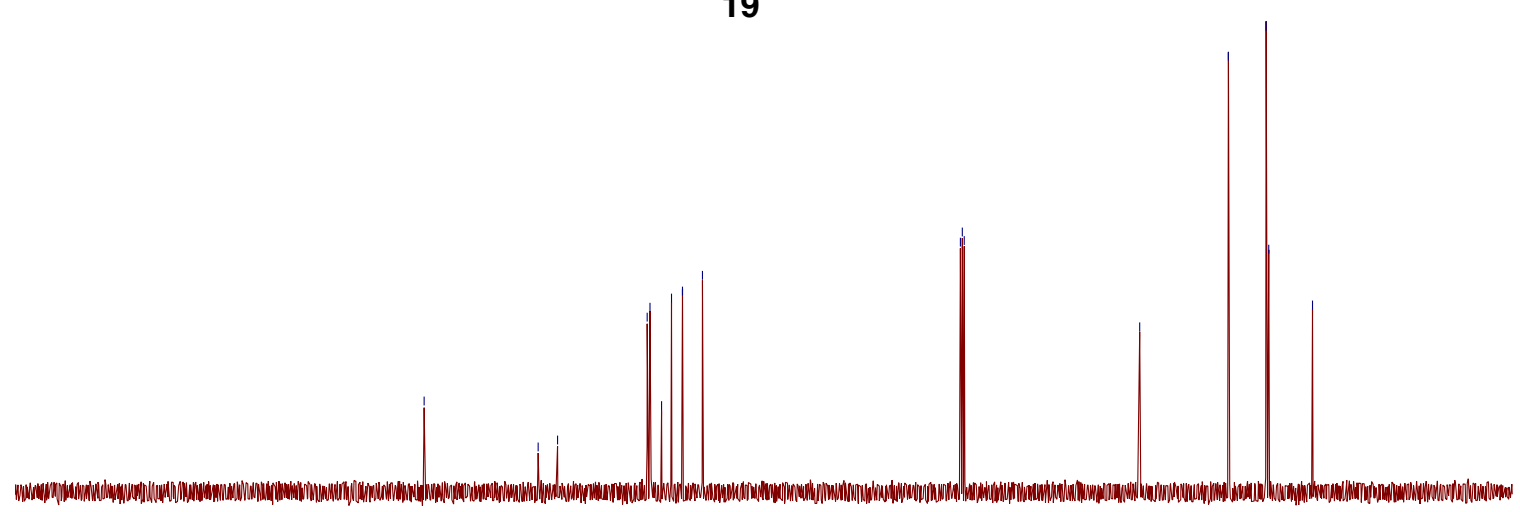

230

$\left.\begin{array}{ccc}120 & 110 \\ \mathrm{f} 1(\mathrm{ppm})\end{array}\right)$ 


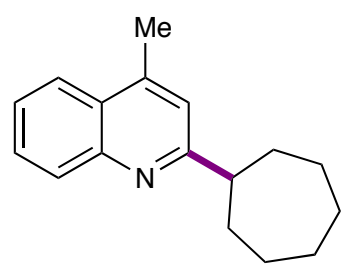

20

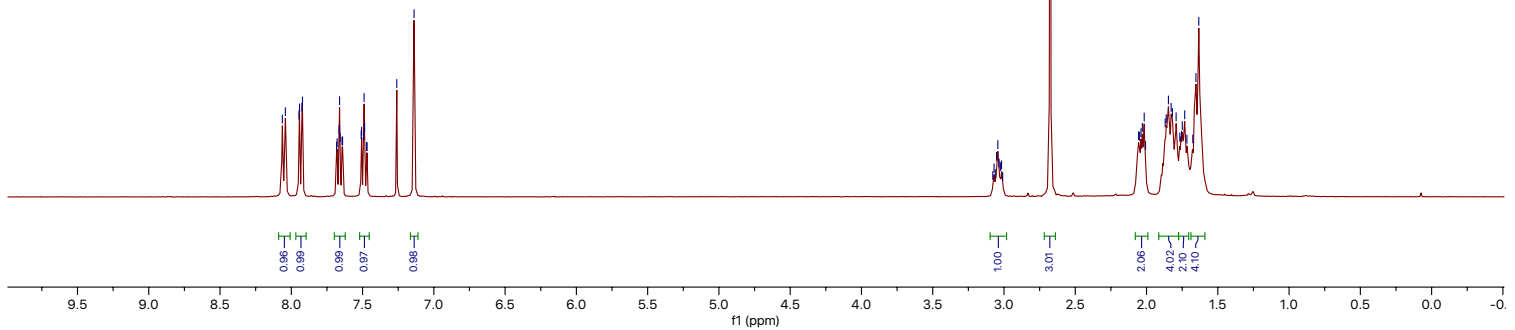

|z-4-32Bp

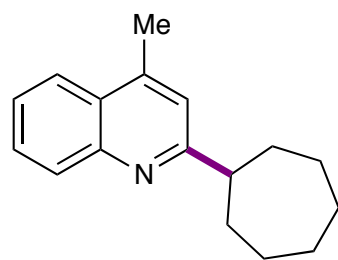

20

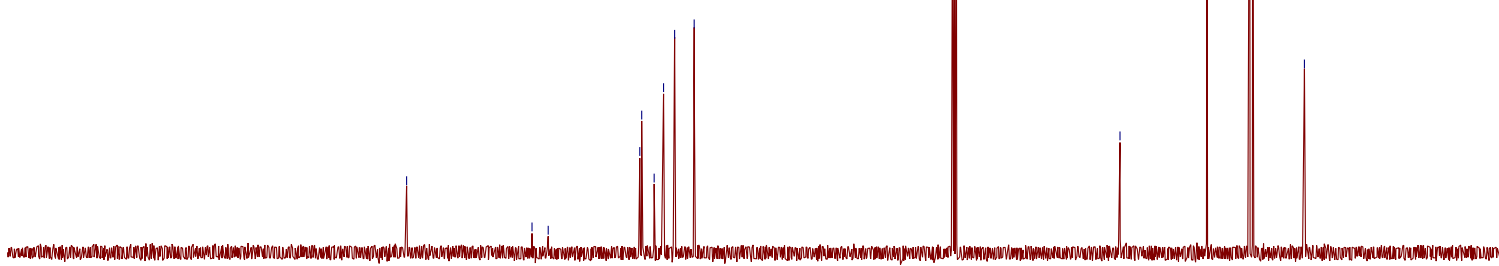

230

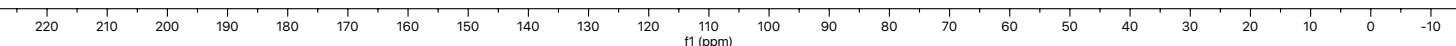


2011746-1z-4-21B-P.10.fidg

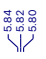

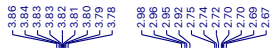

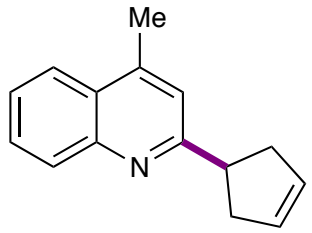

21

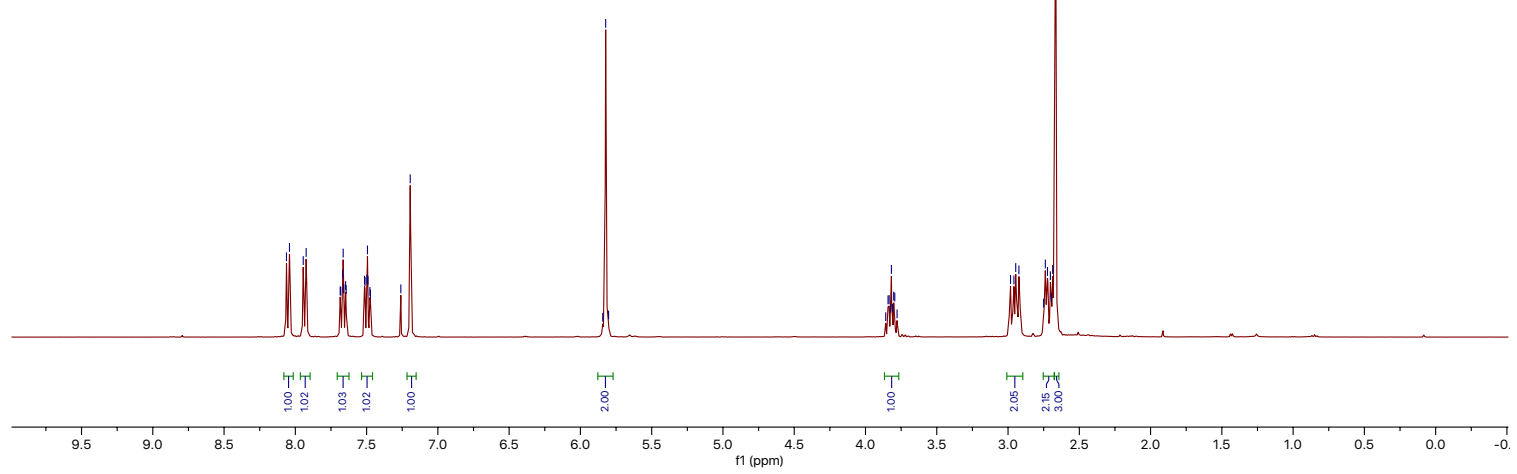

$-1 z-21 b p$

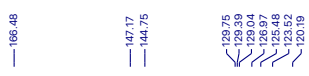

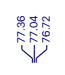

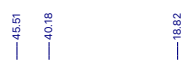<smiles>O=[N+]([O-])c1cc(C2CC=CC2)nc2ccccc12</smiles>

21

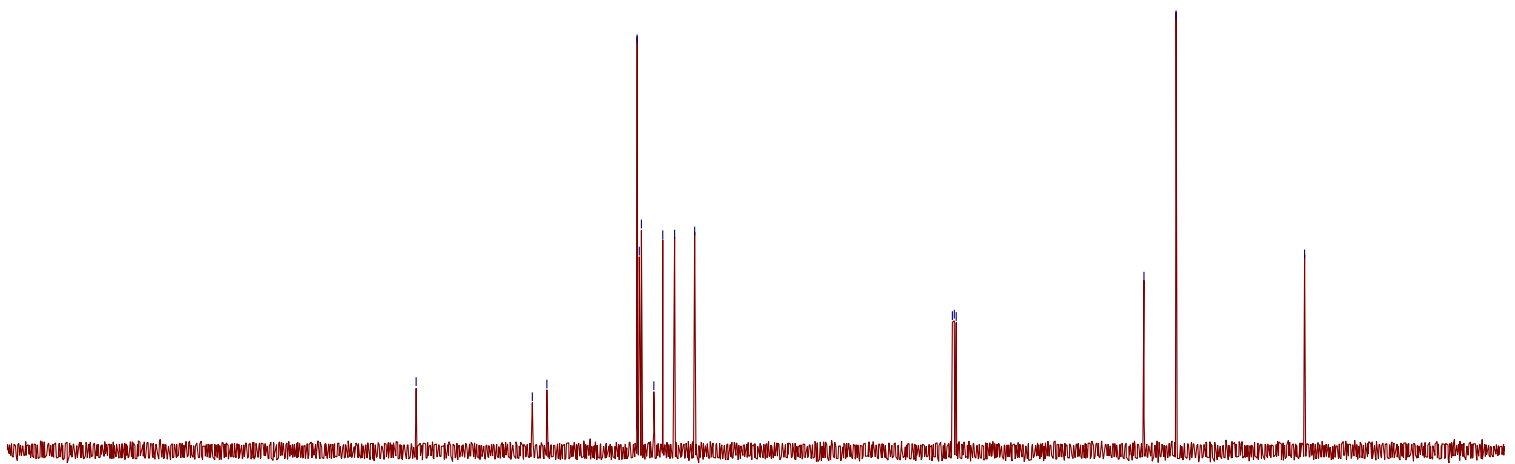

230 $20 \underset{110}{11(p p m)}$ 


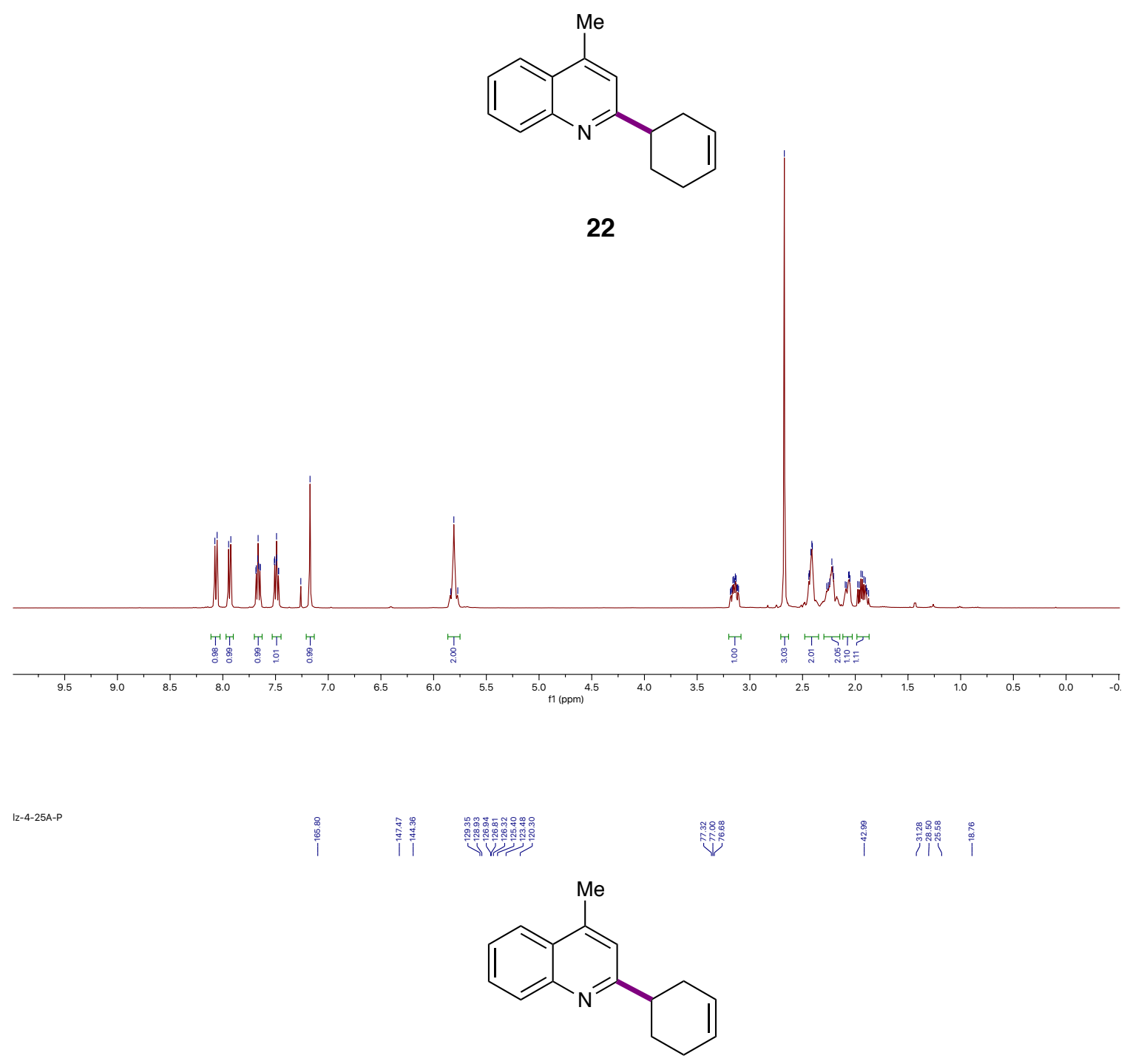

22

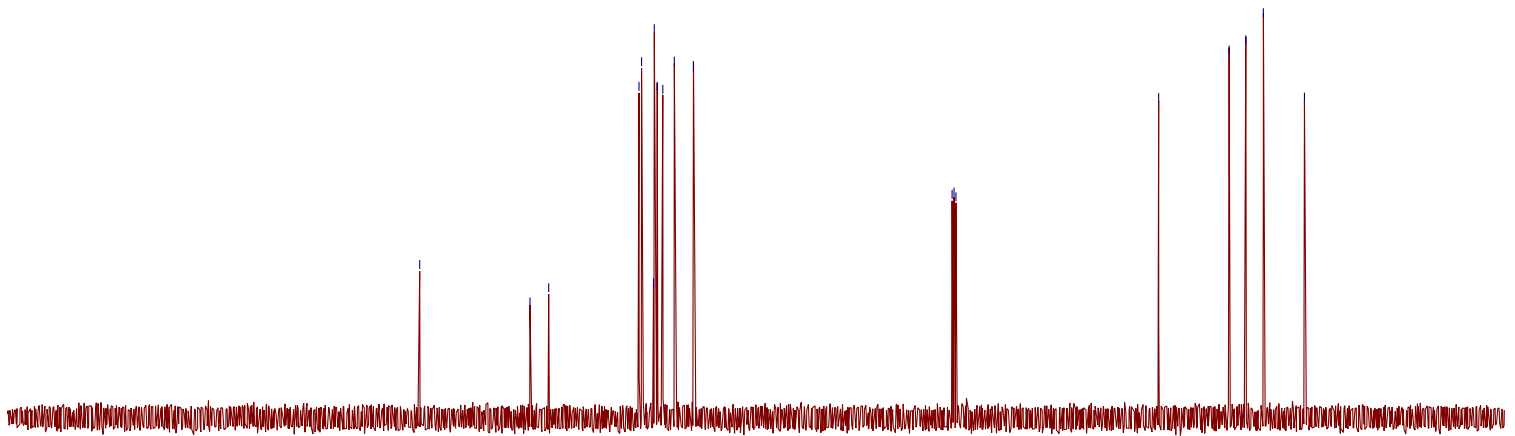

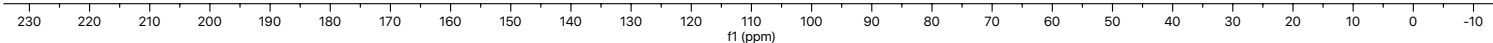



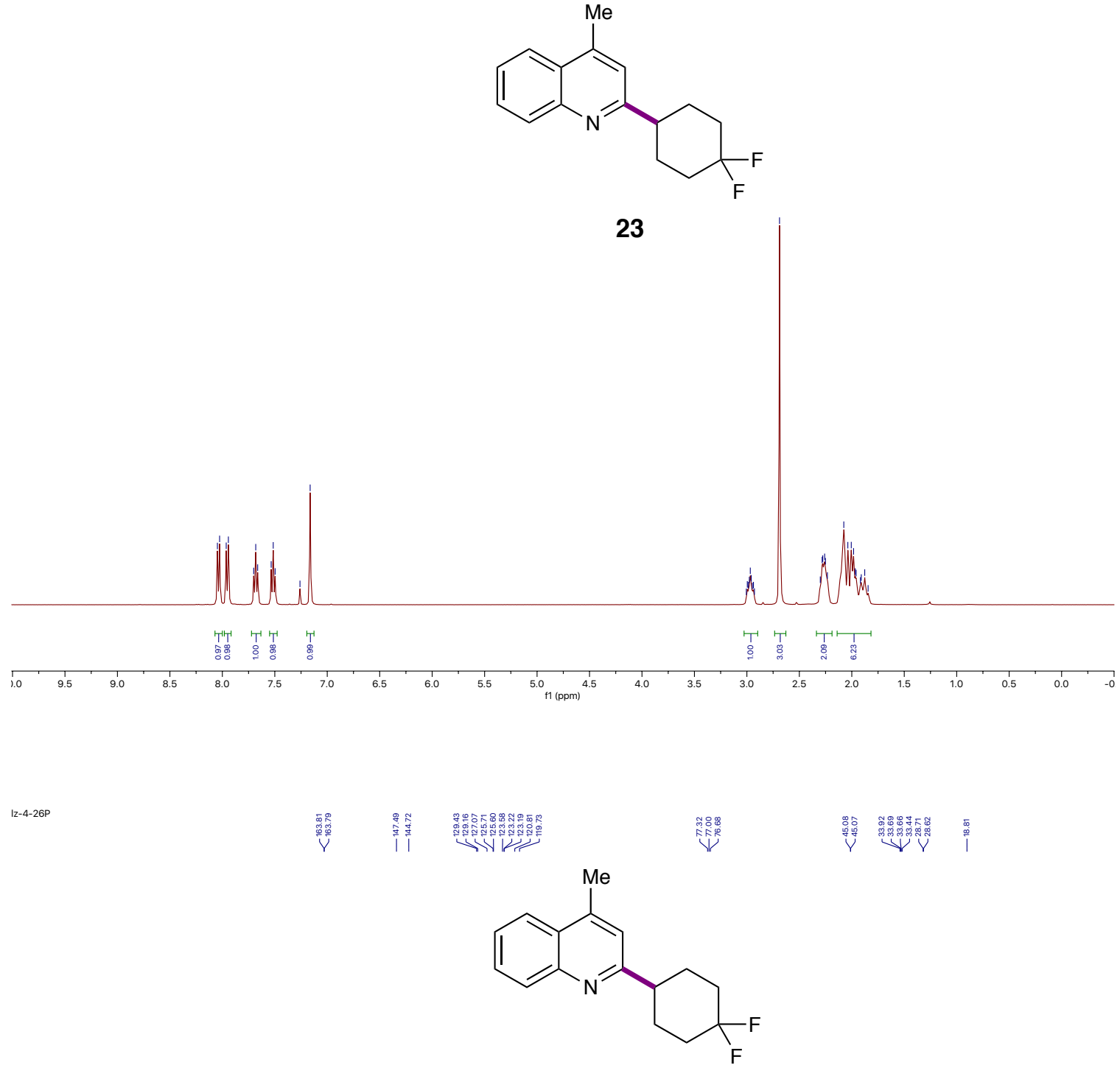

23

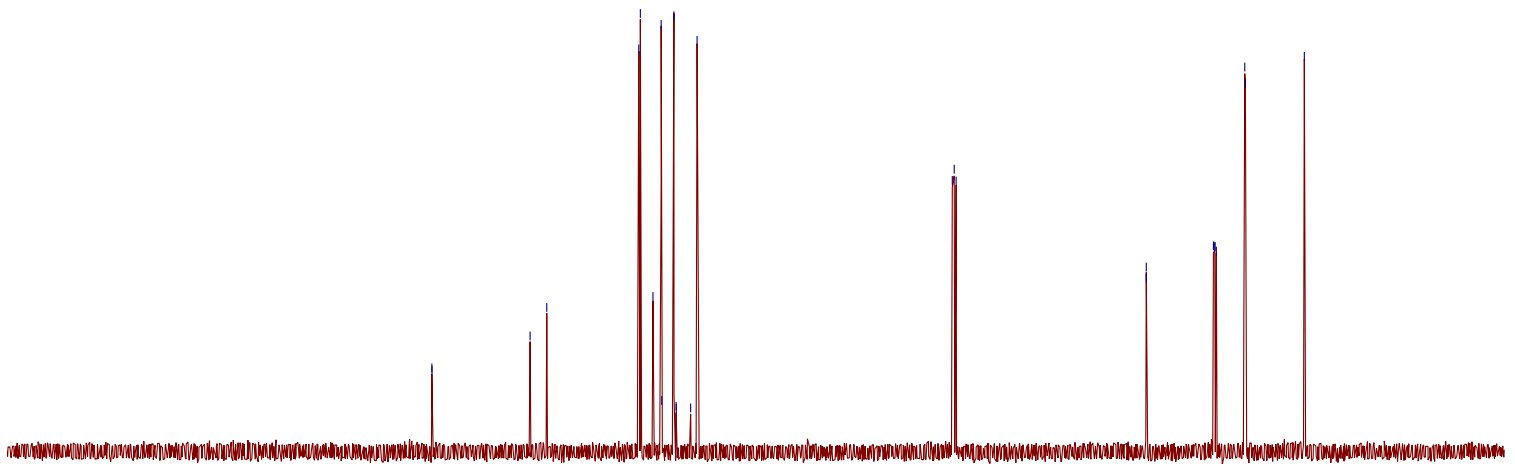

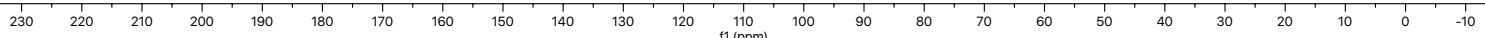


2011746-Iz-4-27-p.10.fid

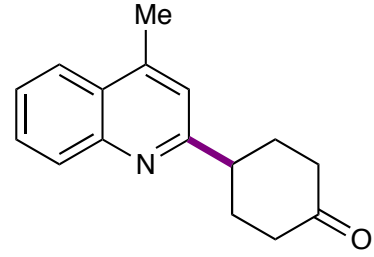

24

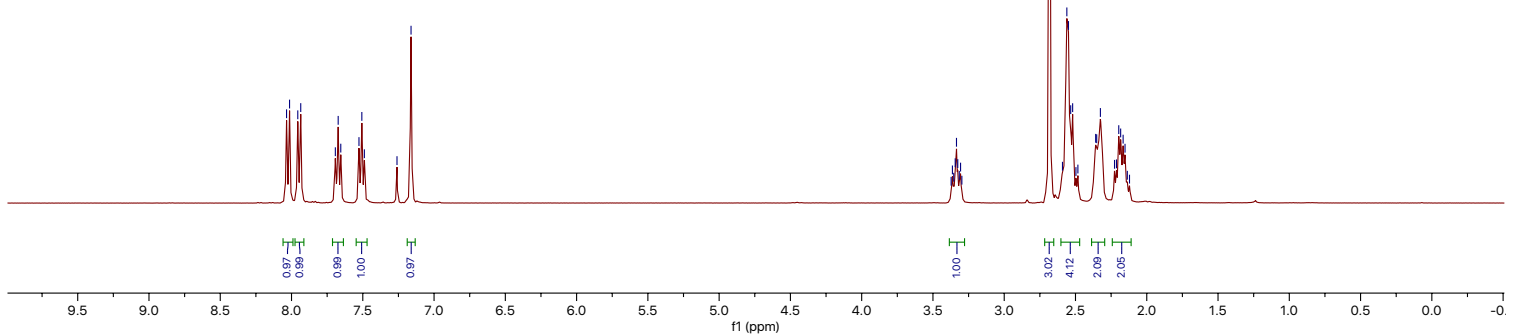

|z-4-27-p
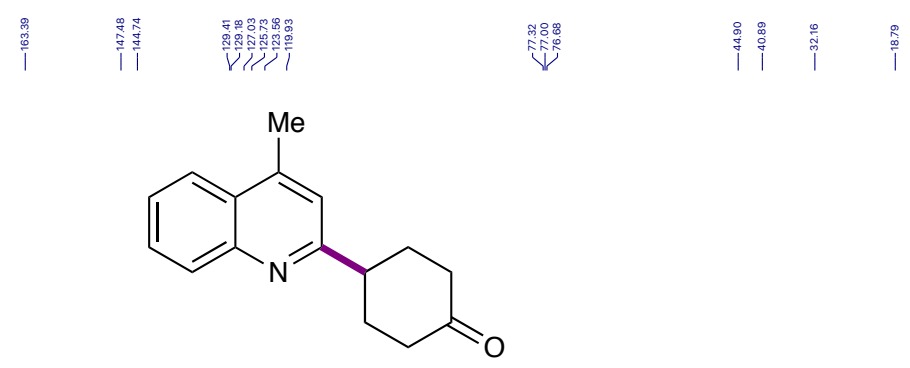

24

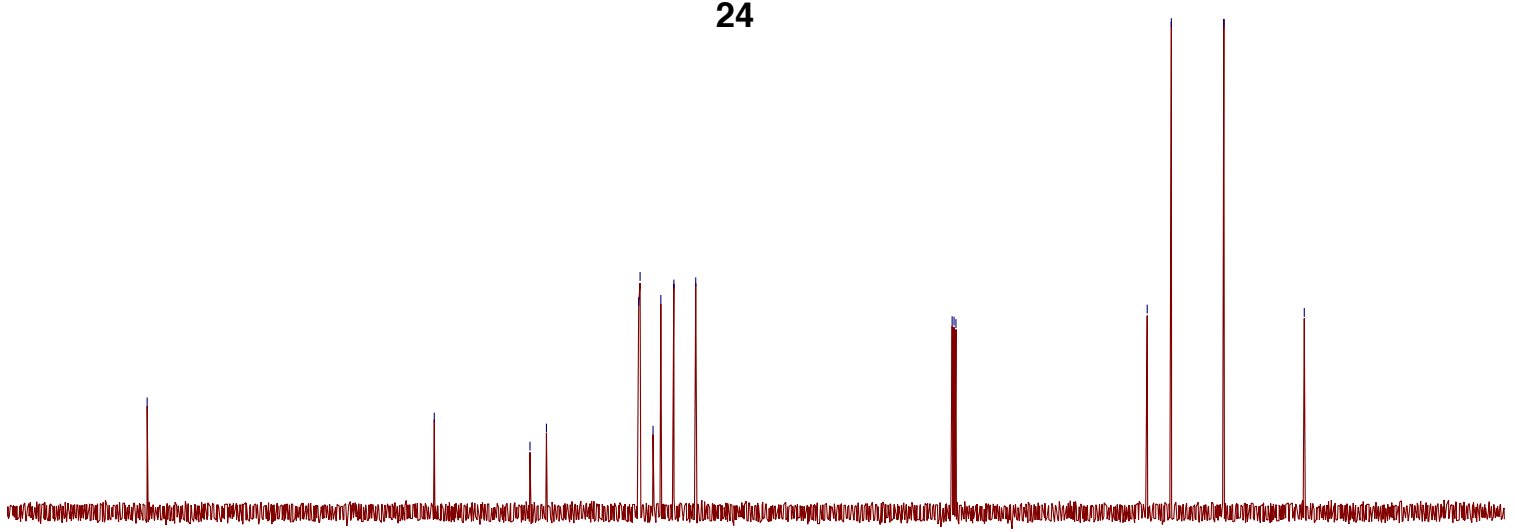

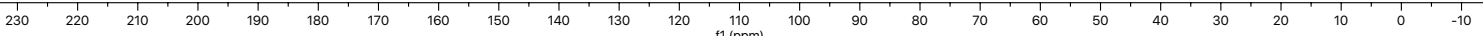




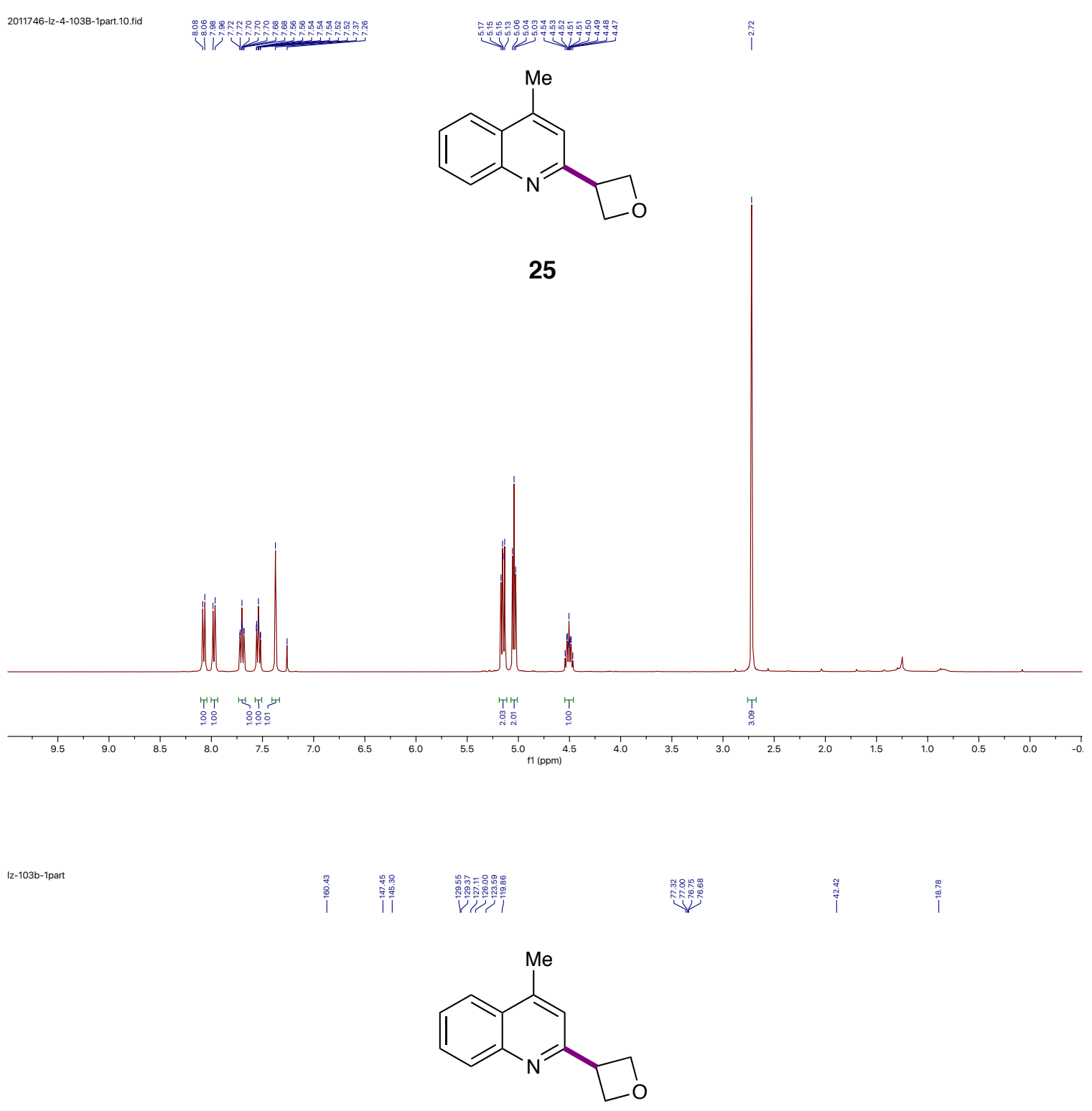

25

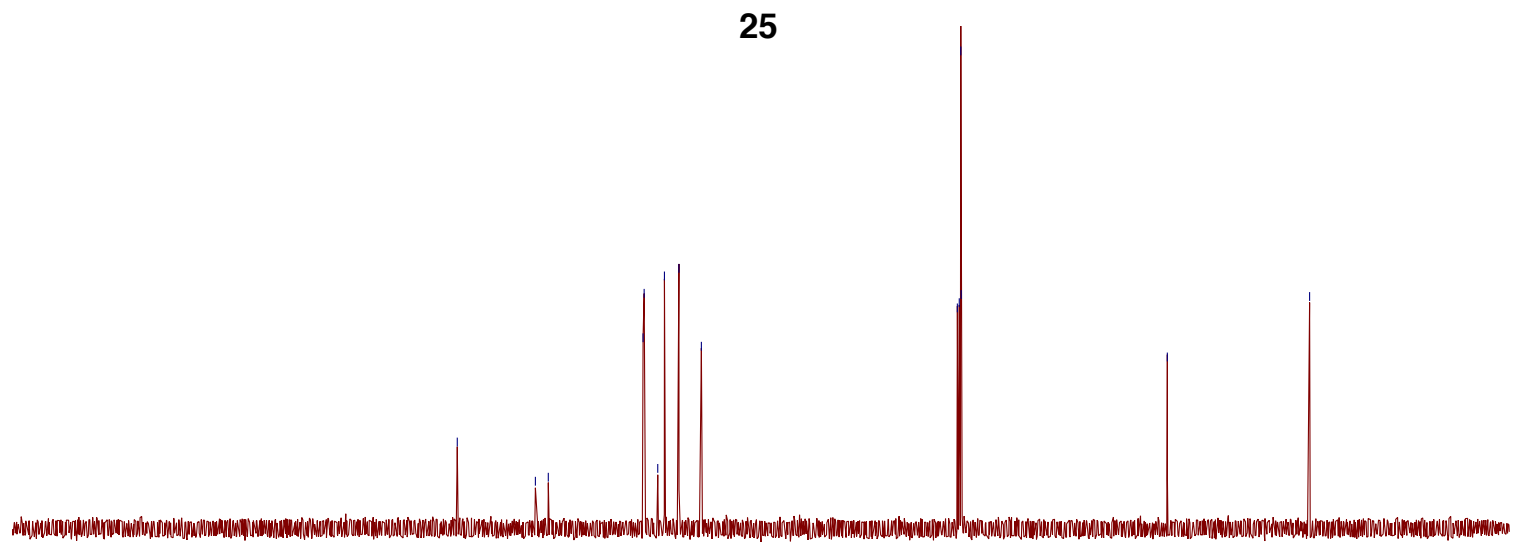

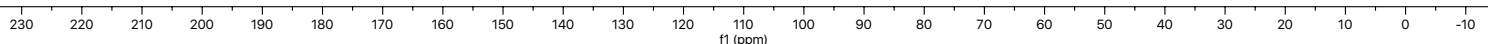




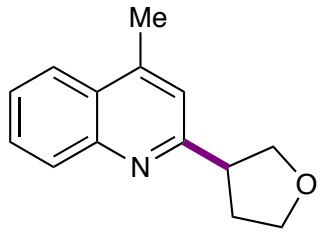

26

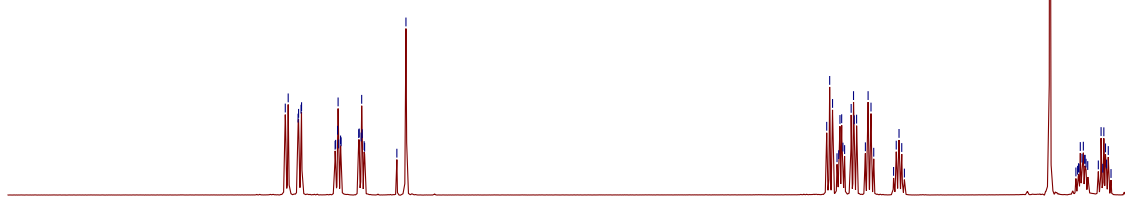

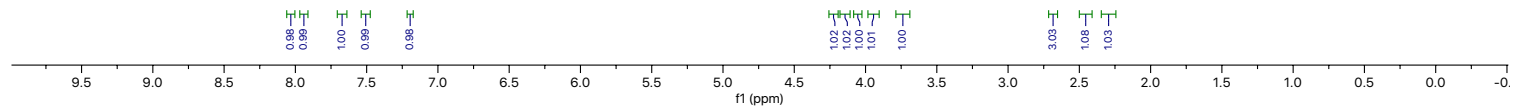

|z-96A-2part<smiles>Cc1cc(C2CCOC2)nc2ccccc12</smiles>

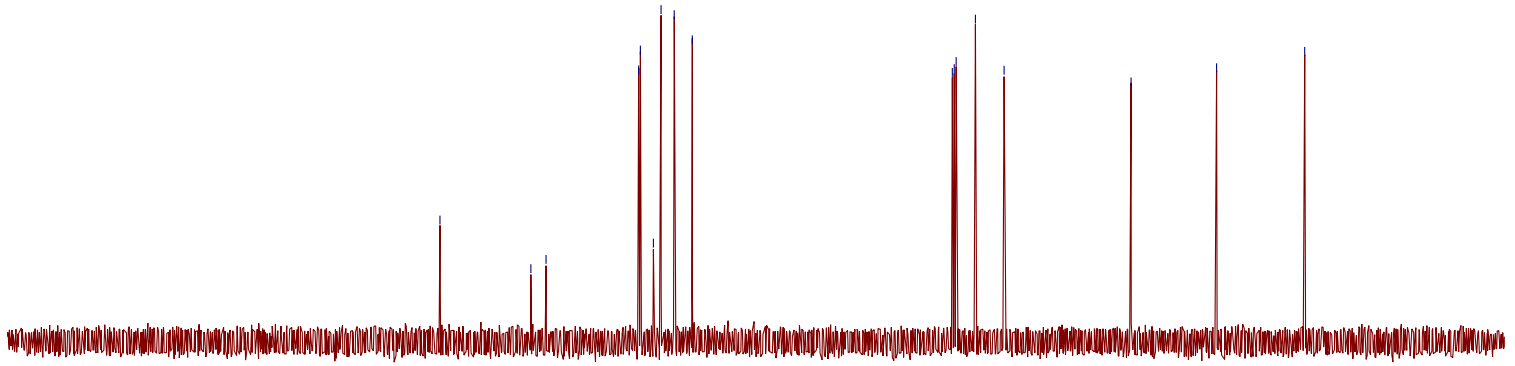

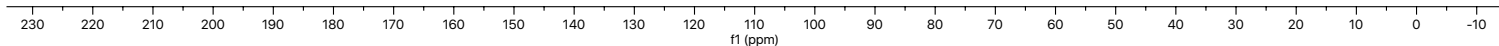




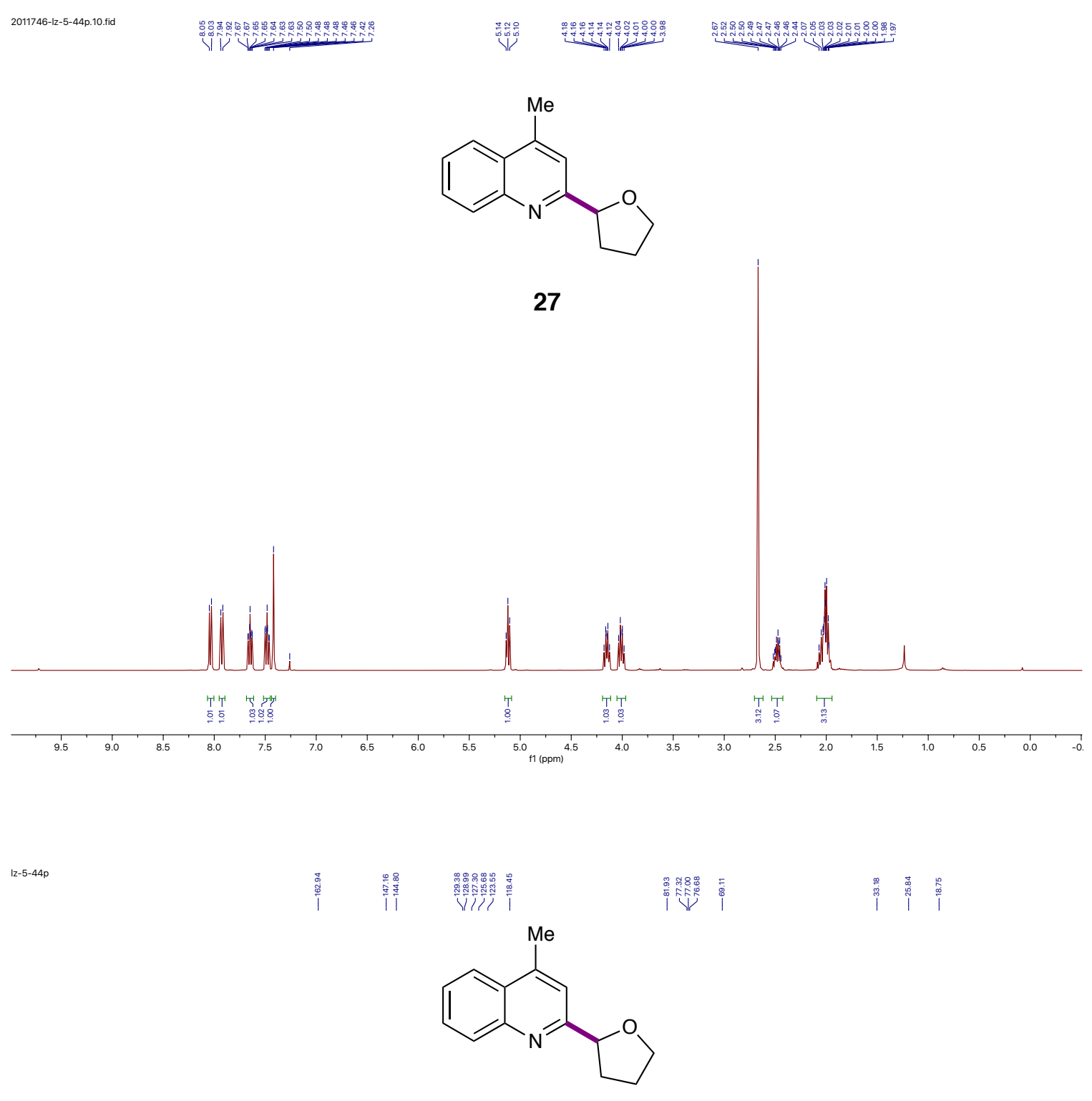

27

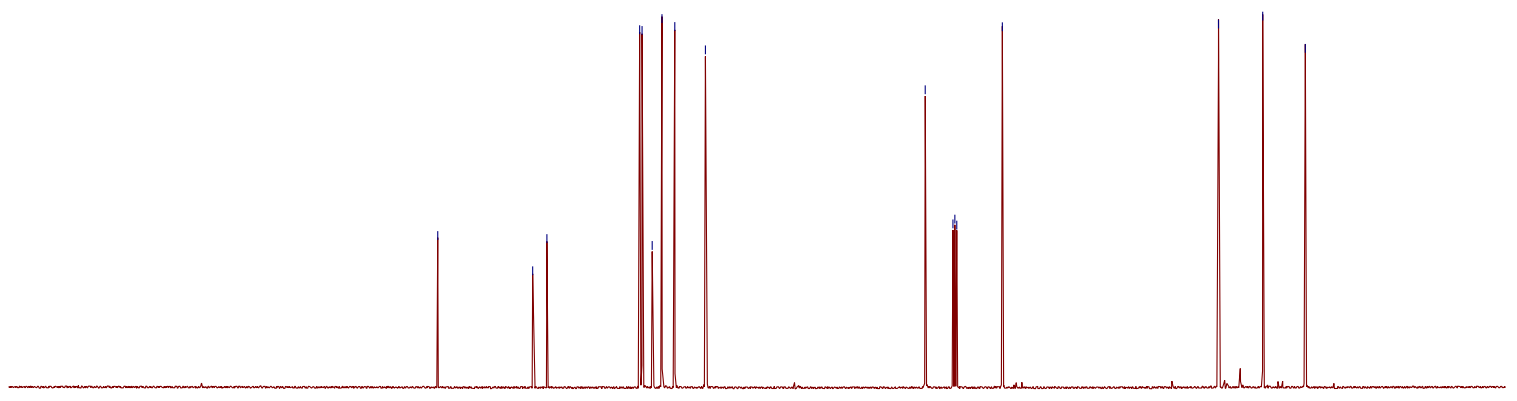

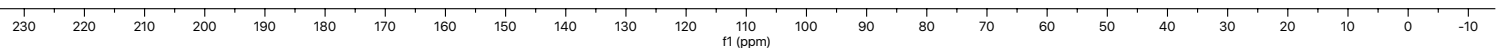




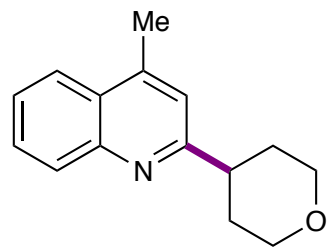

28

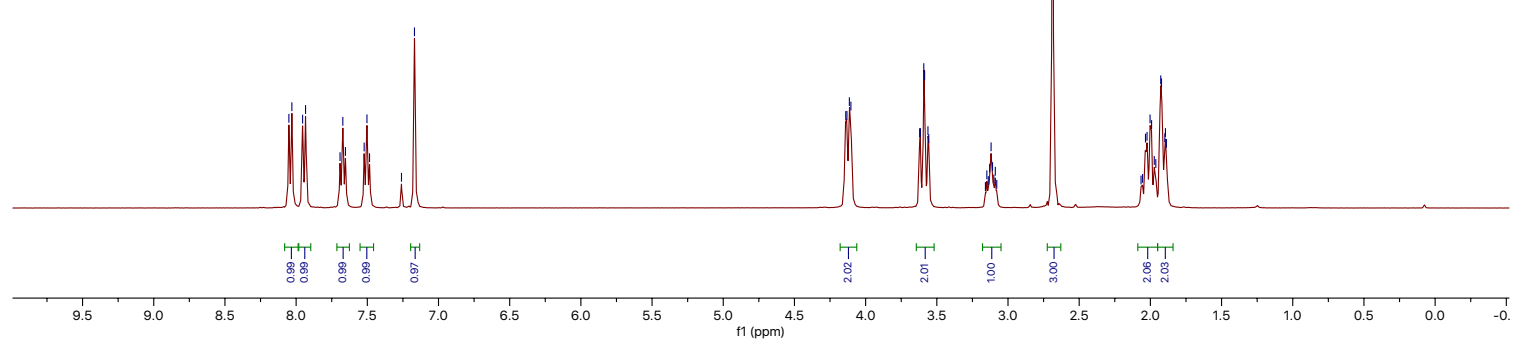

|z-4-31-p<smiles>Cc1cc(C2CCOCC2)nc2ccccc12</smiles>

28

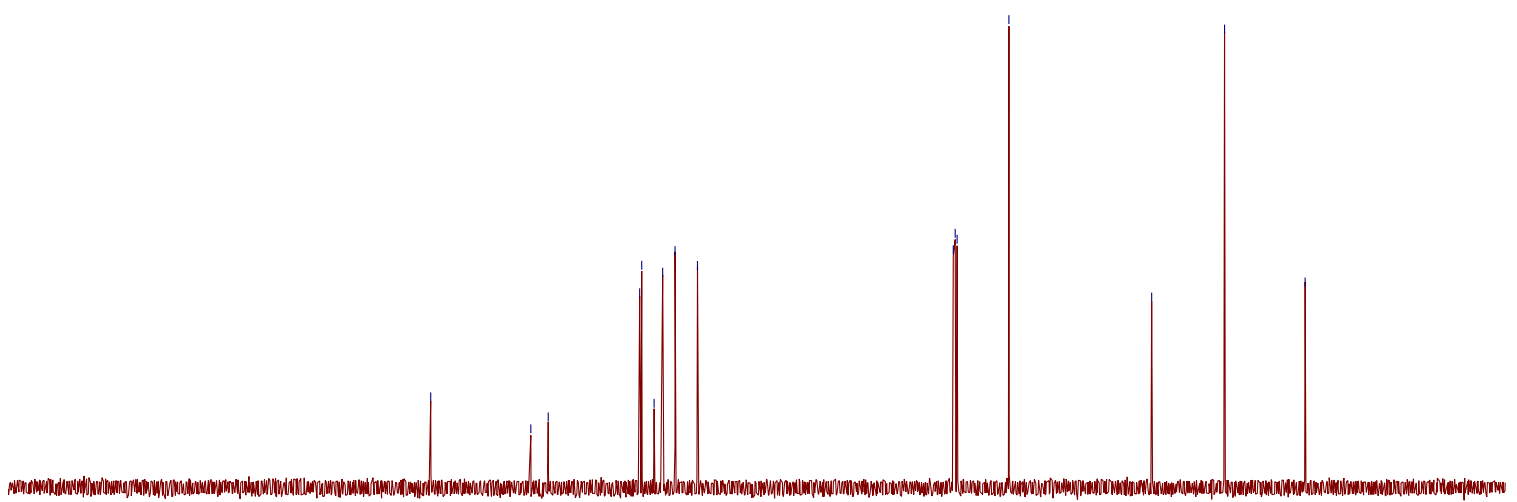




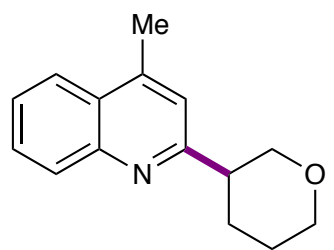

29

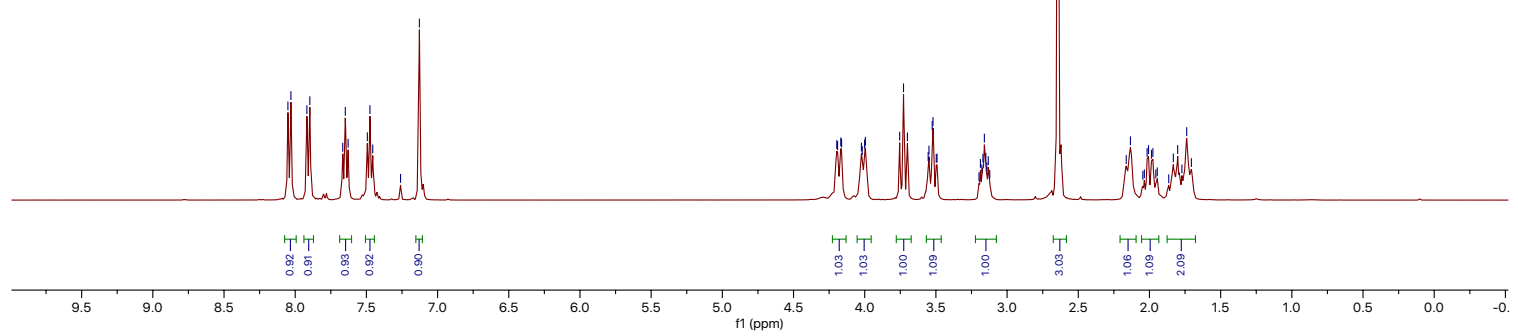

|z-4-30-p
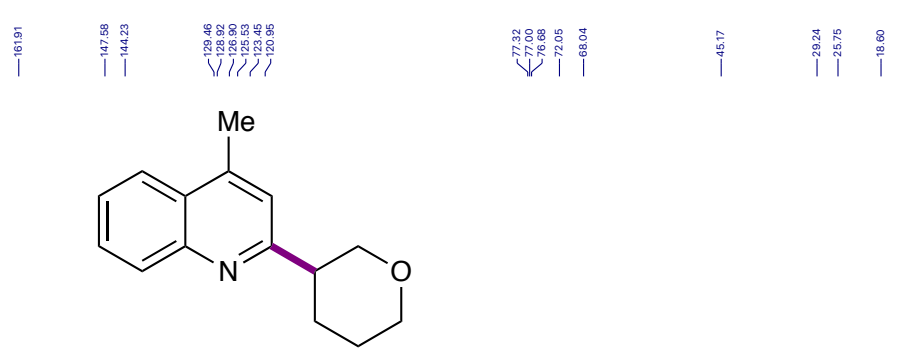

29

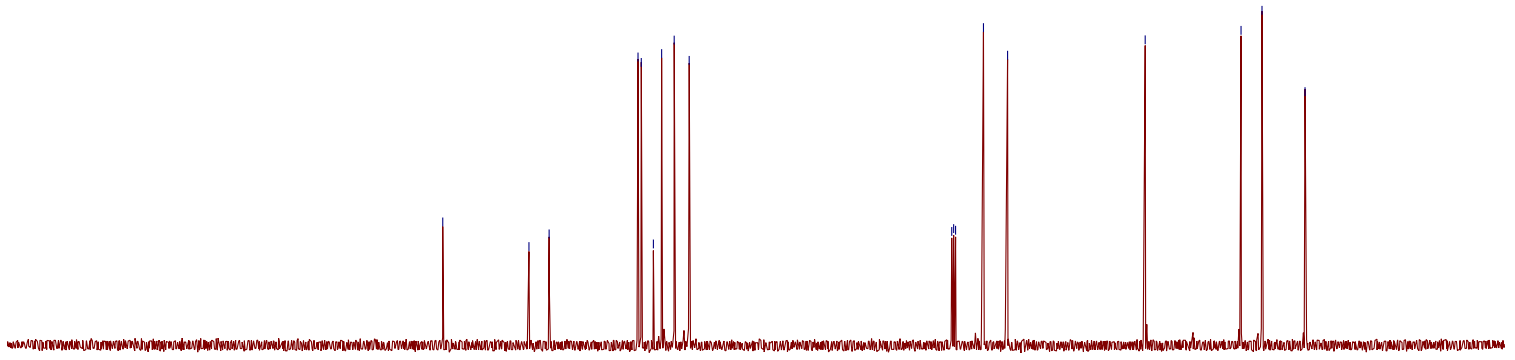

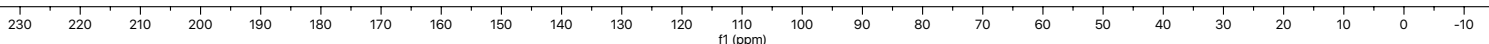


2011746-Iz-4-29-p. 10

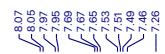

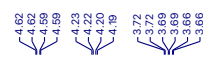

ì

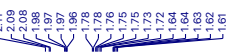<smiles>O=[N+]([O-])c1cc(C2CCCCO2)nc2ccccc12</smiles>

30

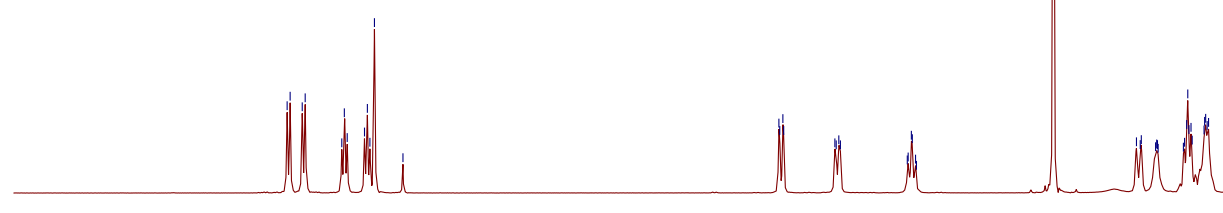

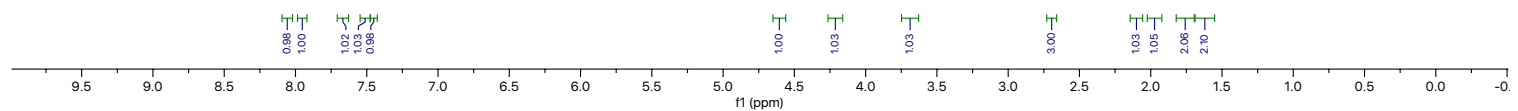

|z-4-29-p

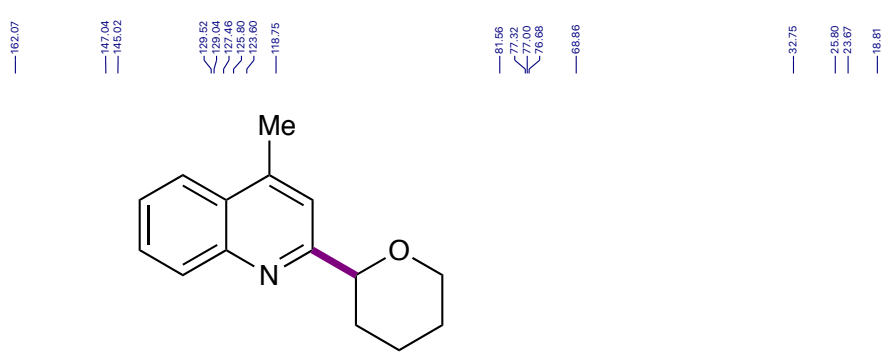

30

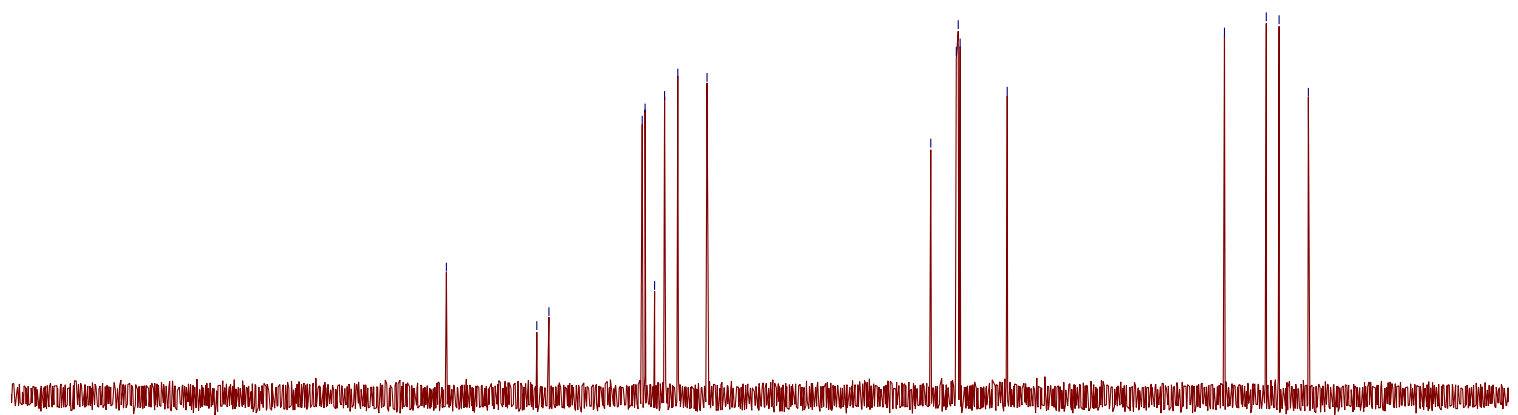

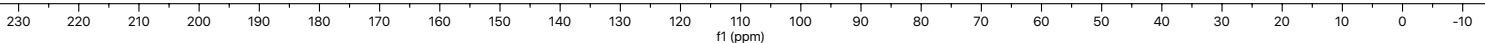




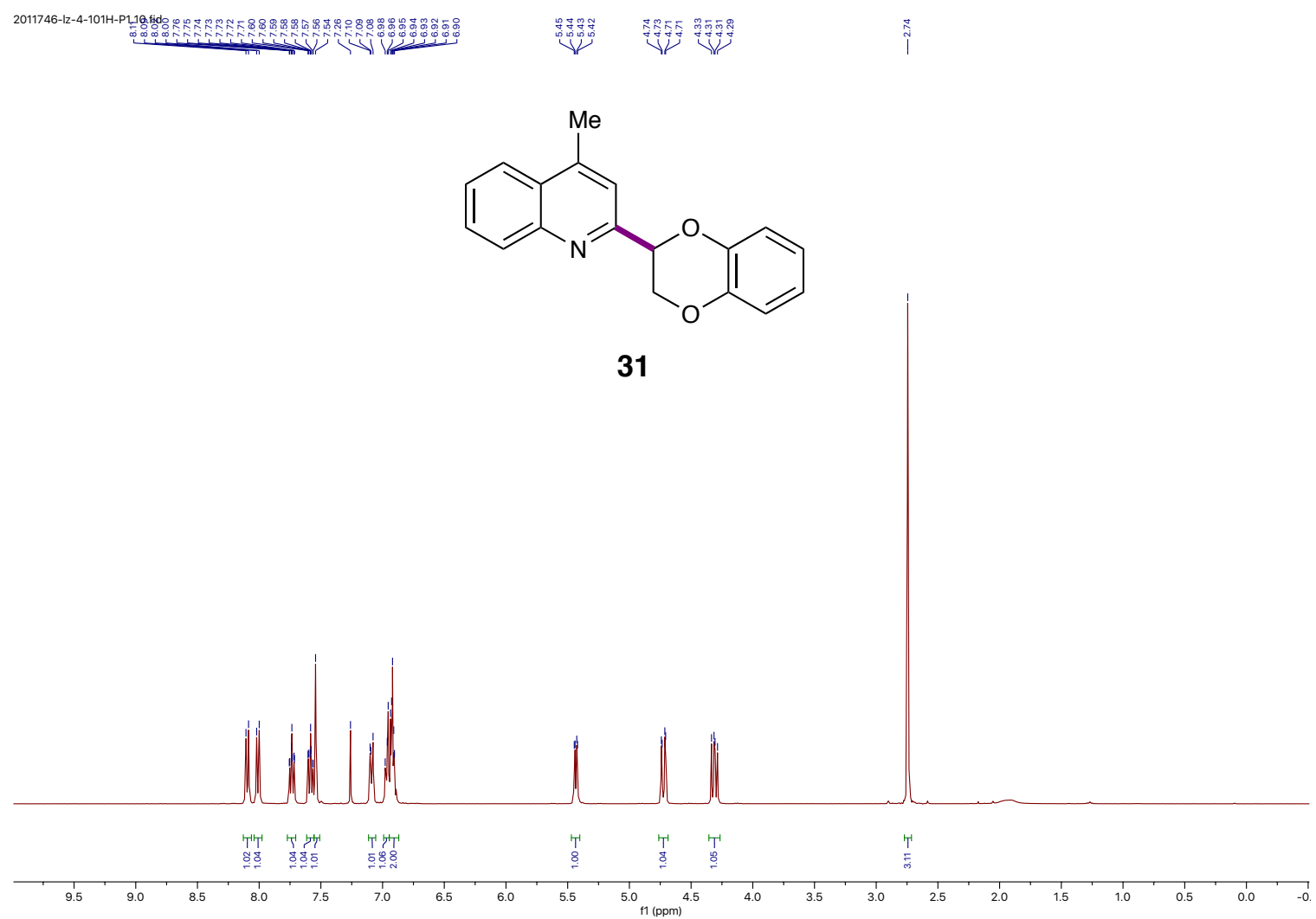

$-\mid z-4-101+-P 1$

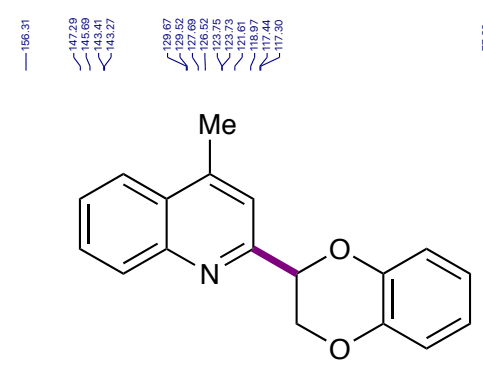

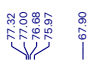

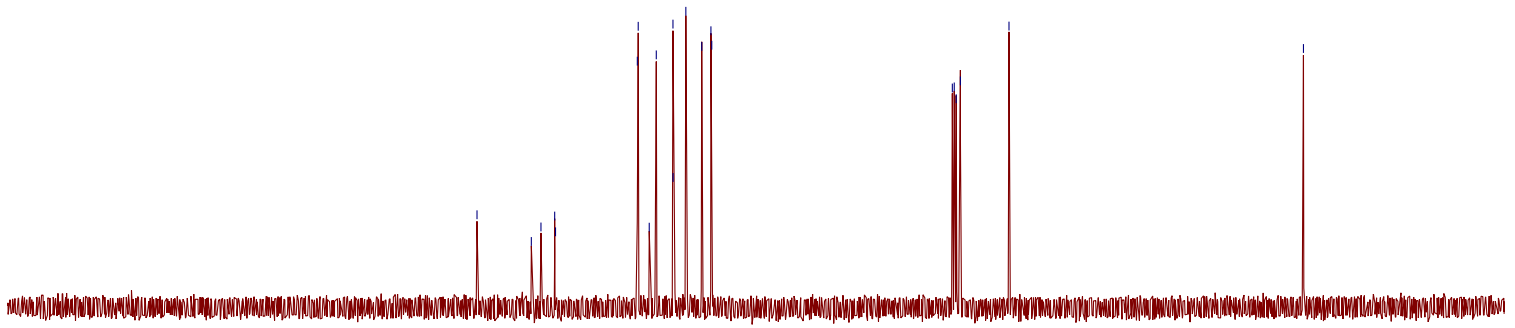

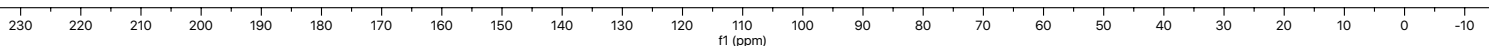




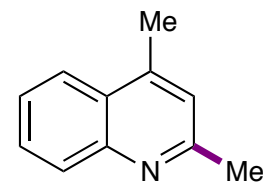

32

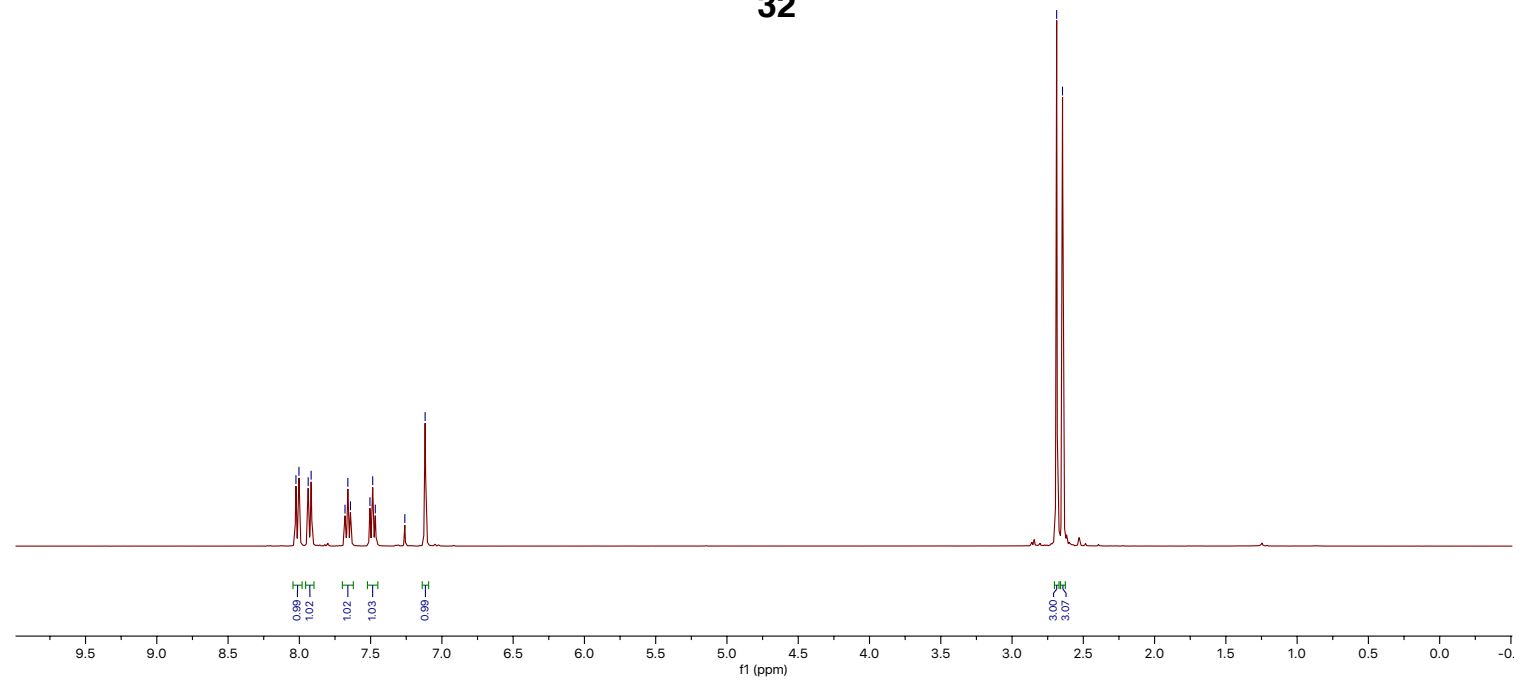

|z-|xx-p<smiles>Cc1cc([N+](=O)[O-])c2ccccc2n1</smiles>
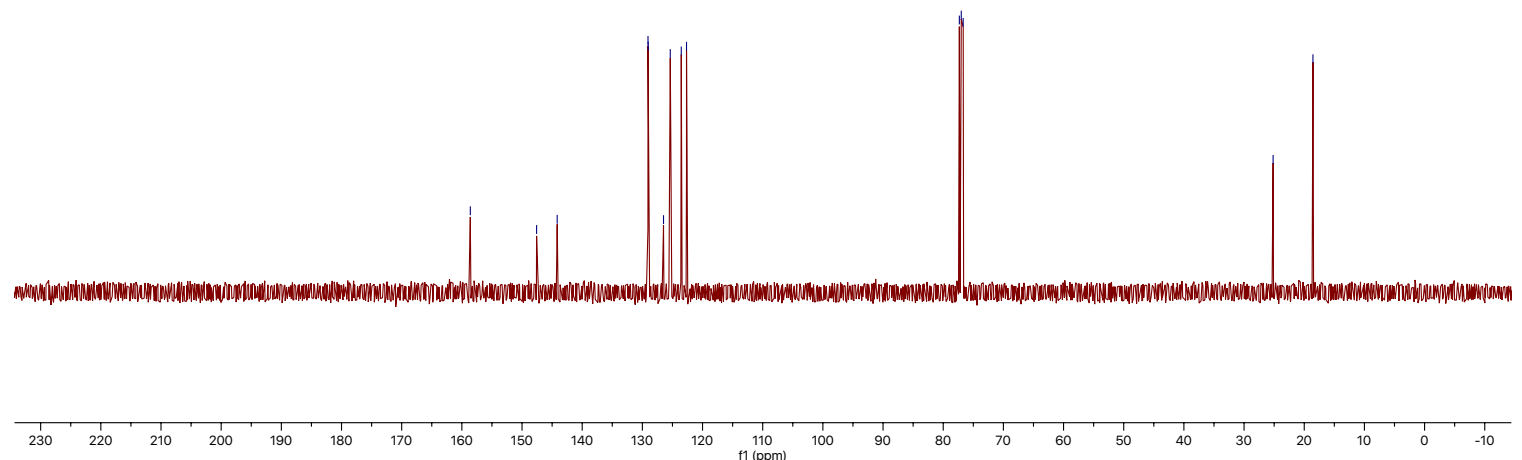


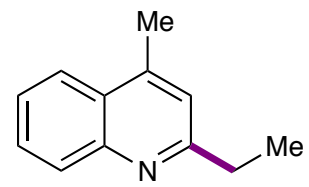

33

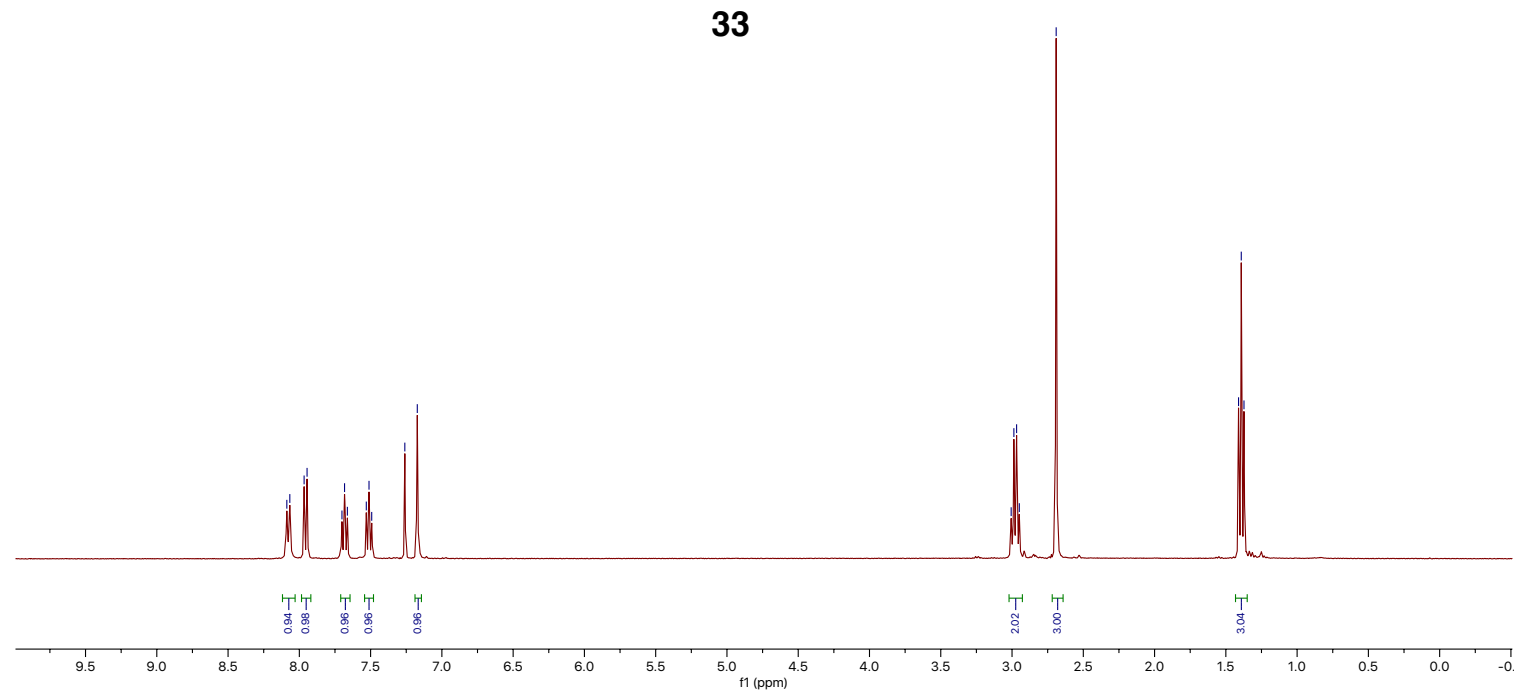

$|z-4-2 c-p|$
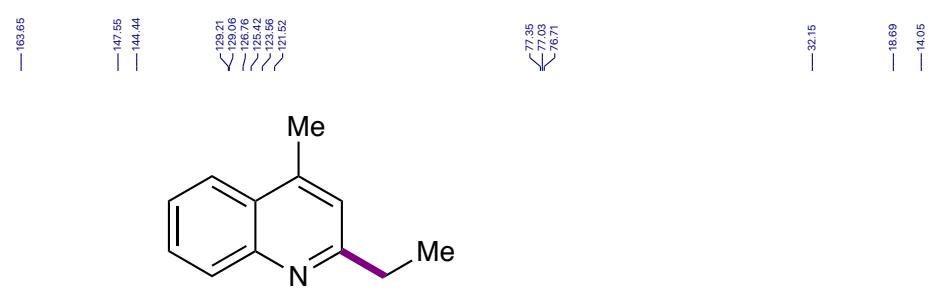

33

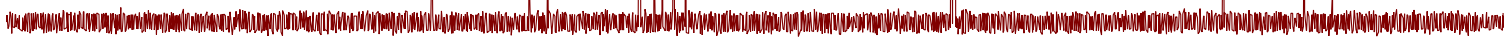

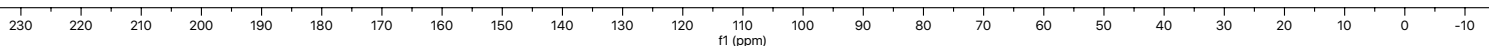




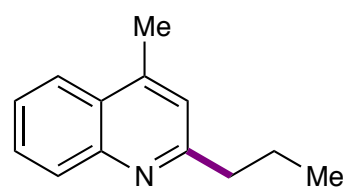

34

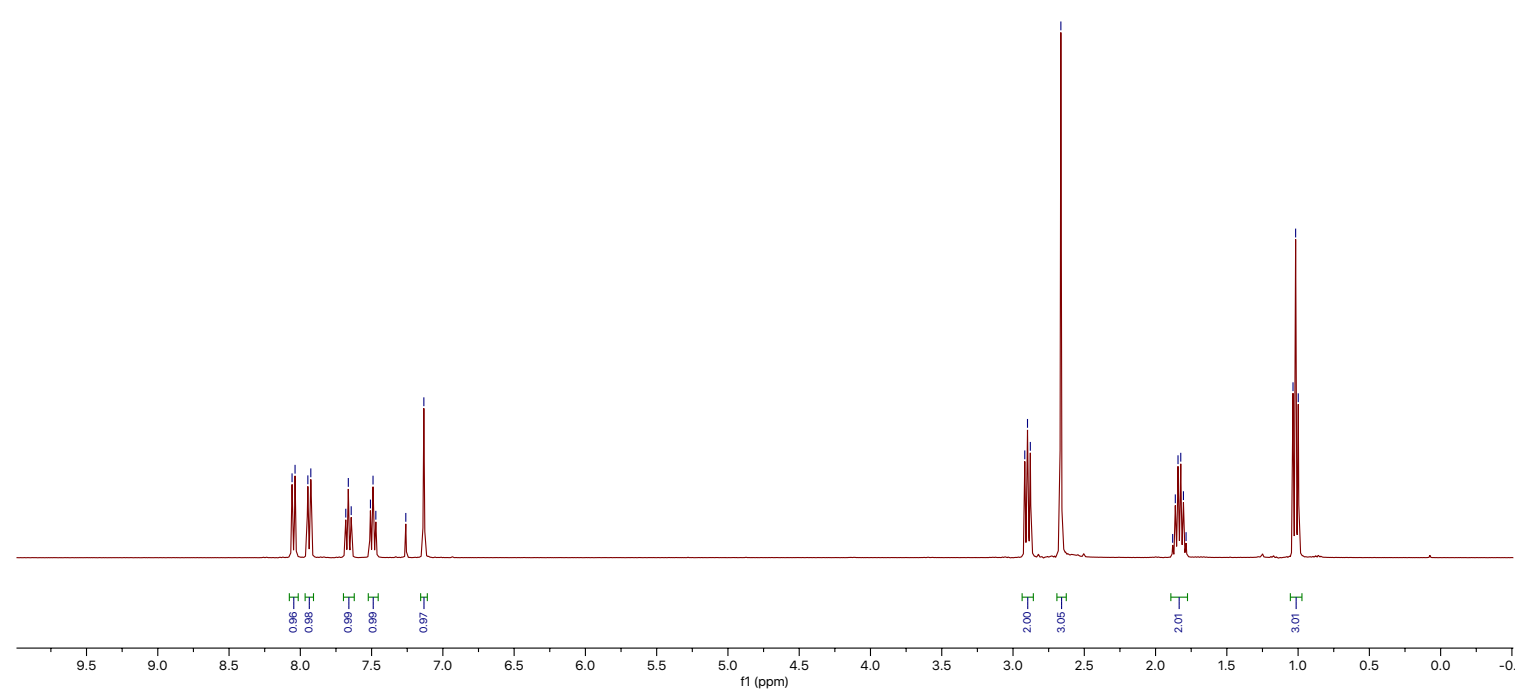

Iz-4-4B-P1

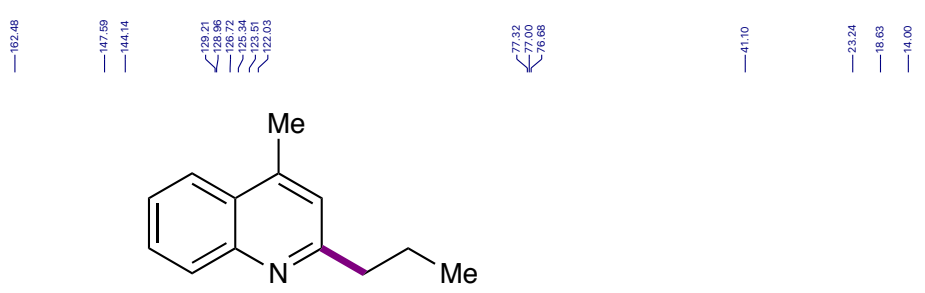

34

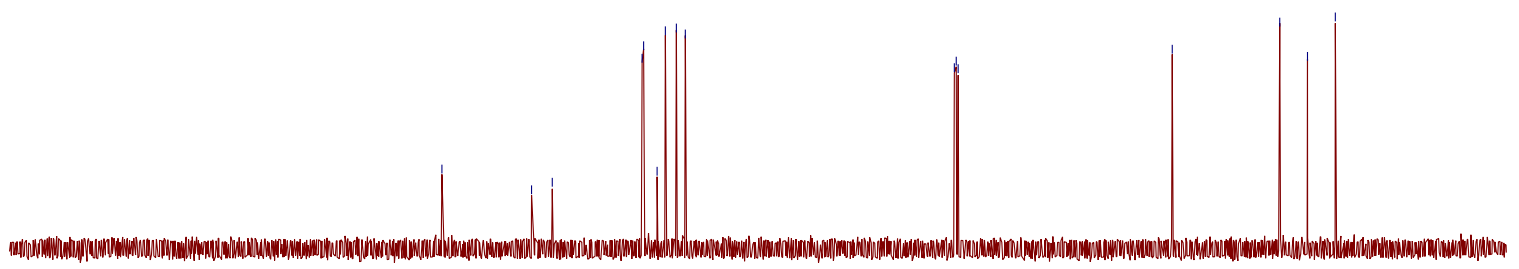

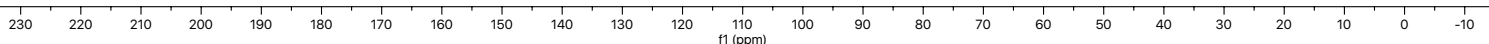


2011746-1z-5-5P.10.fid

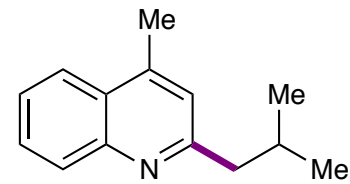

35

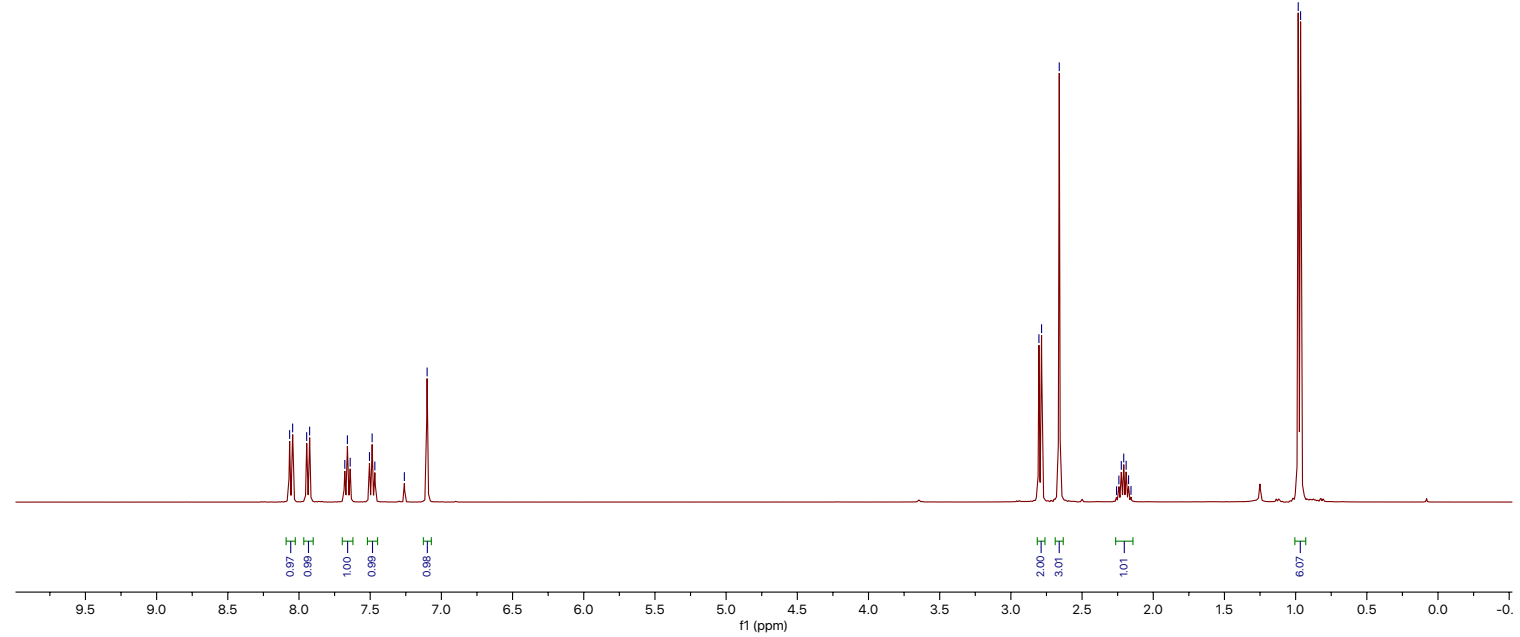

|z-4-5ap1

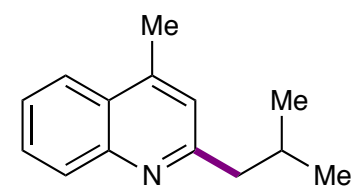

35

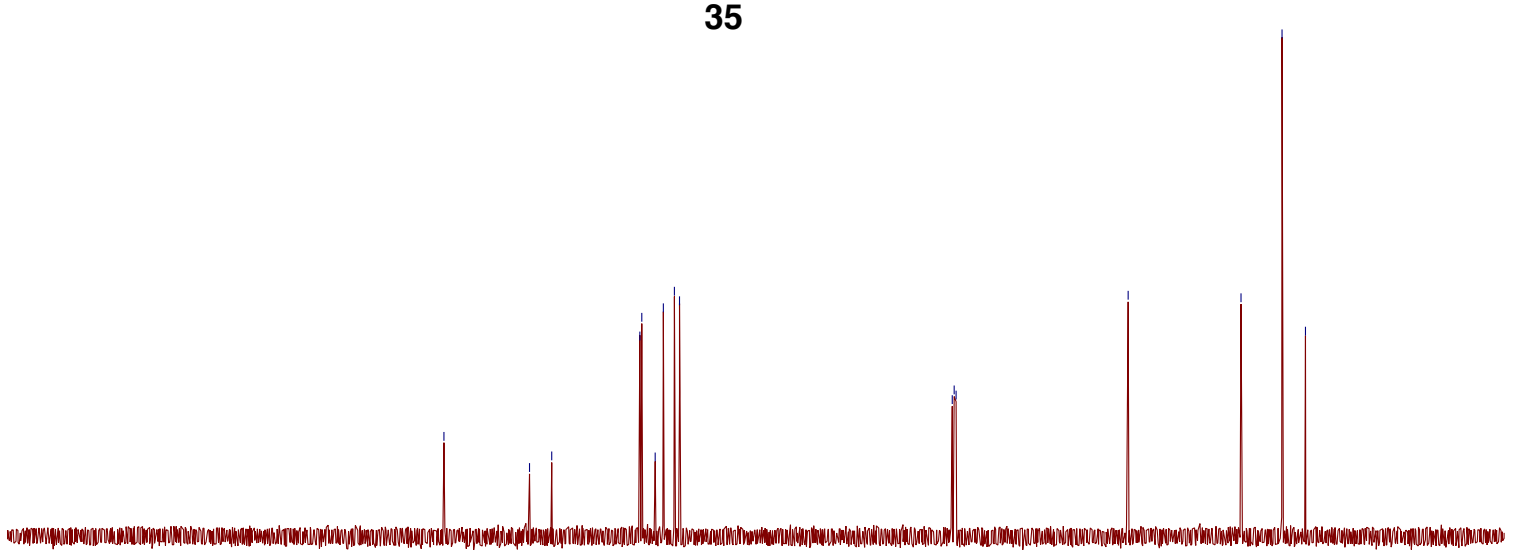

230

110
$\mathrm{f1}(\mathrm{ppm})$ 


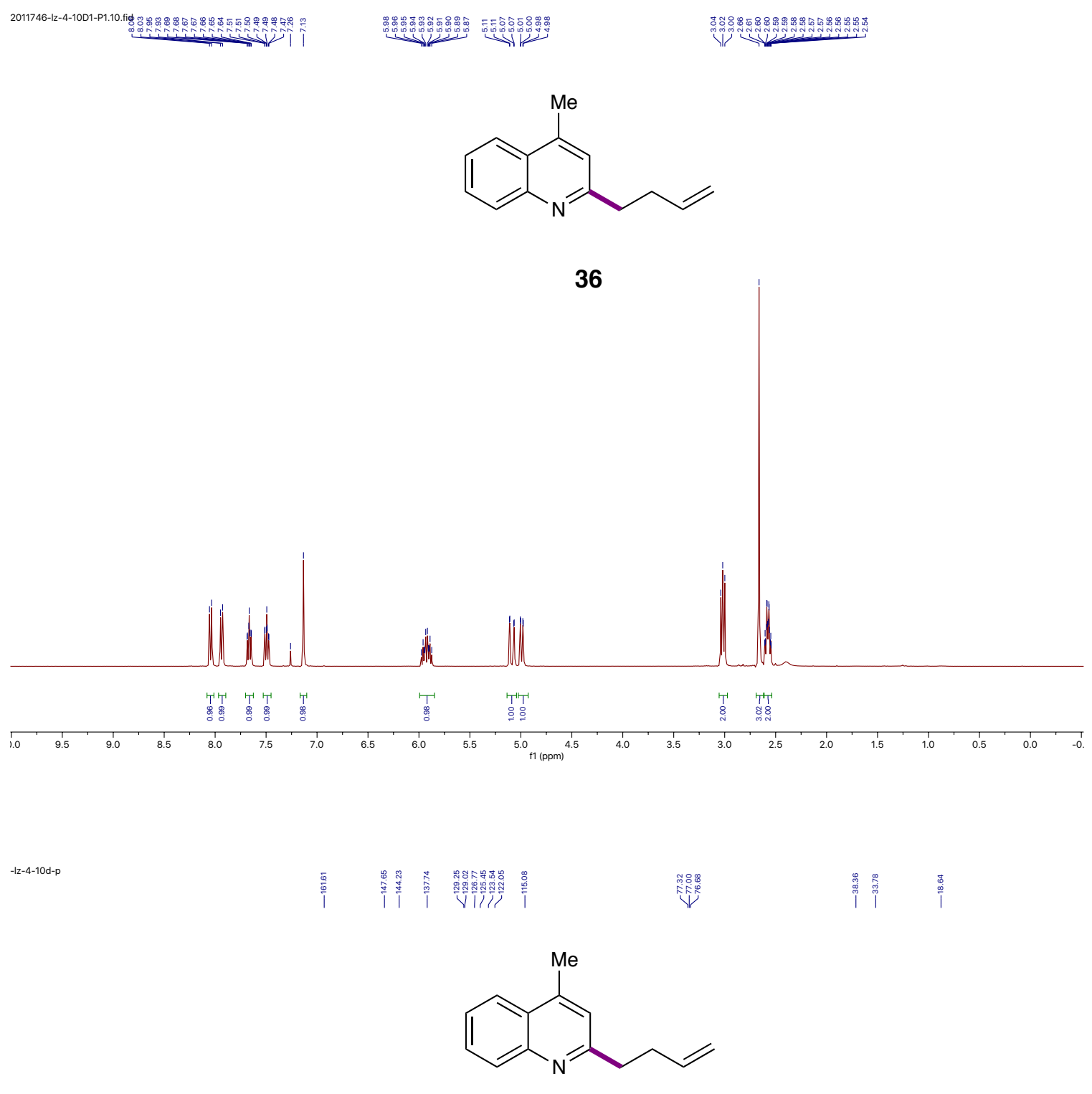

36

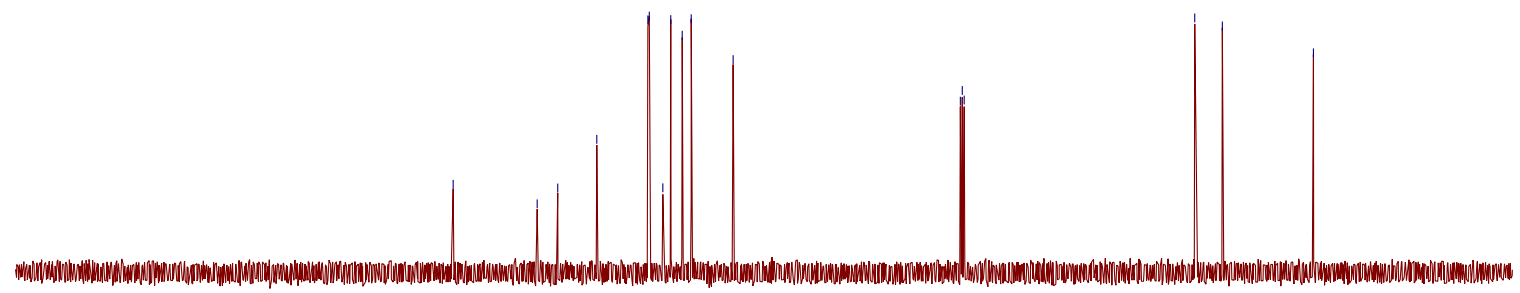

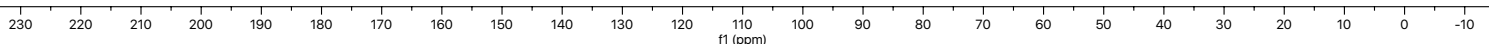




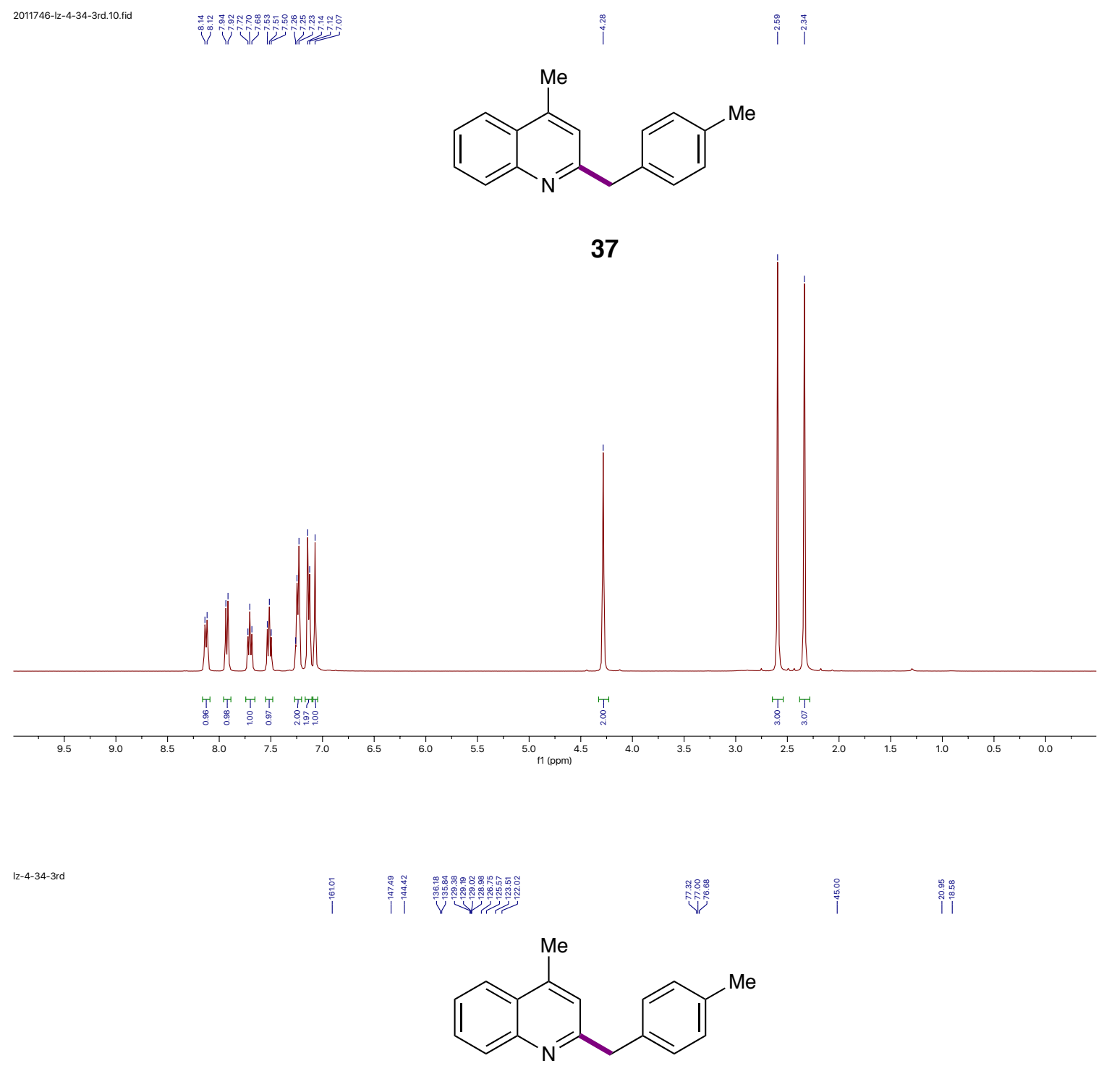

37

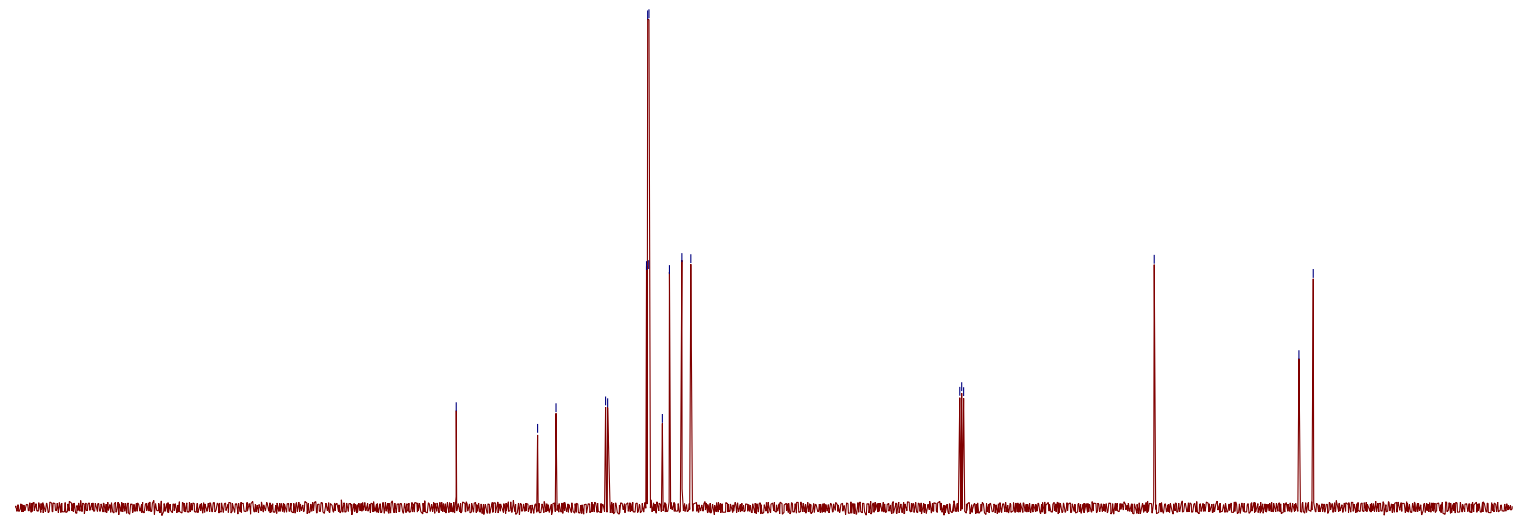

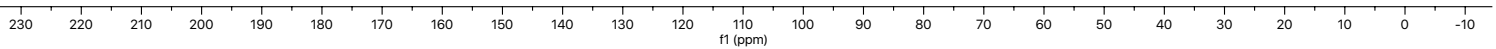



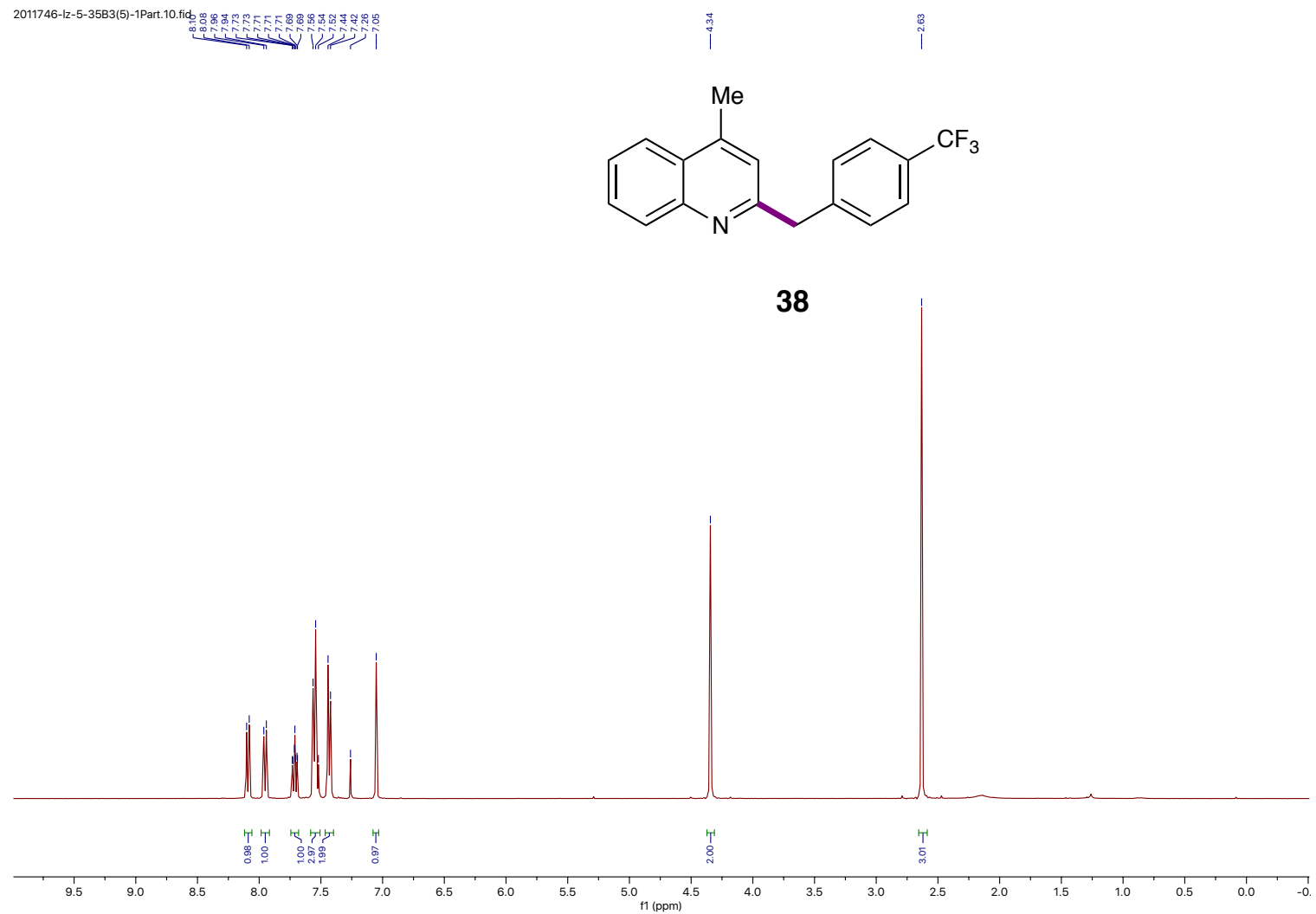

|Z-4-35A-P1
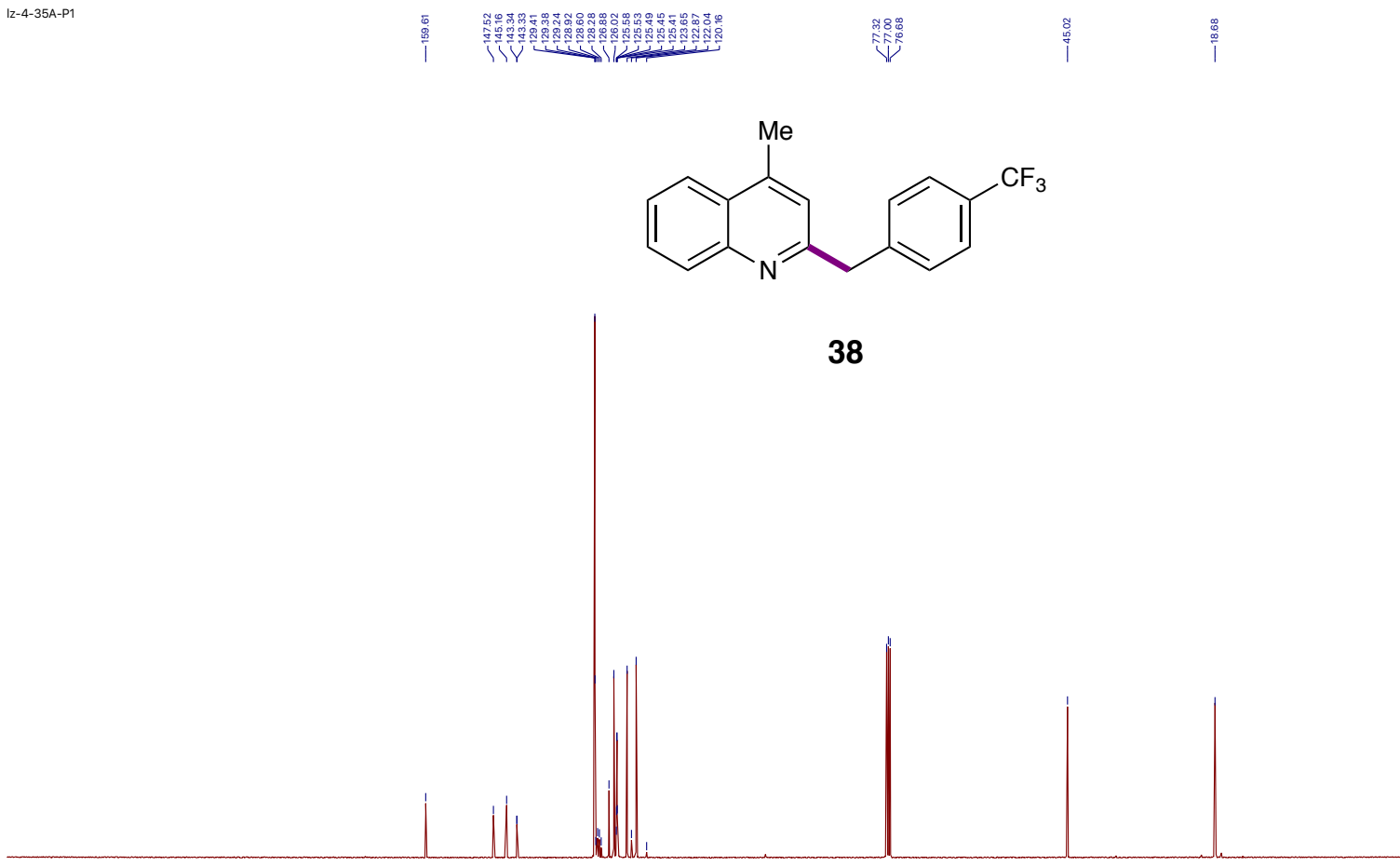

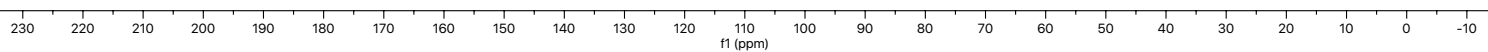




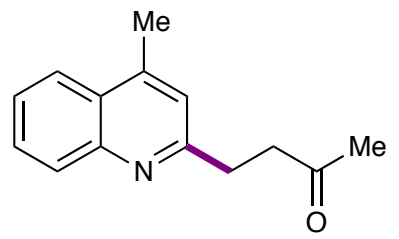

39

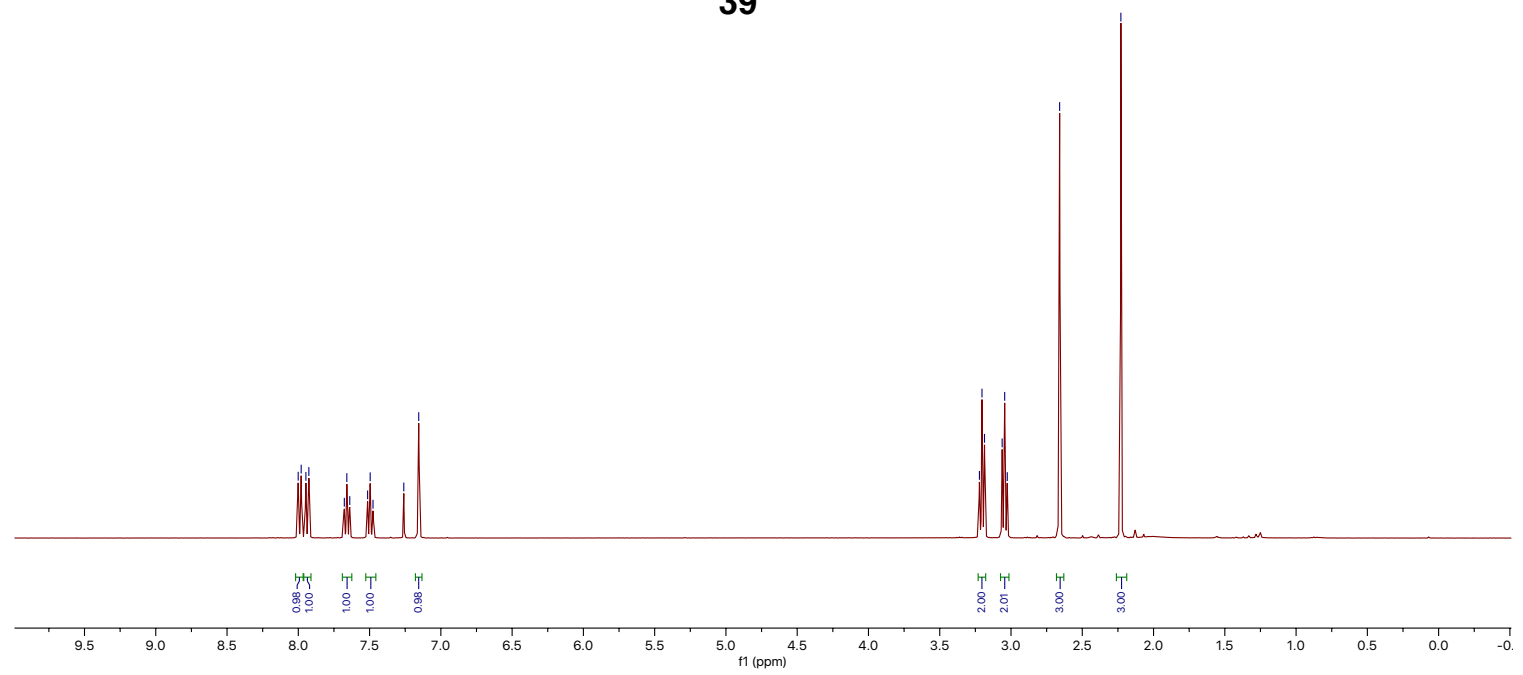

$-\mid z-4-12-p$<smiles>CC(=O)CCc1cc([N+](=O)[O-])c2ccccc2n1</smiles>

39

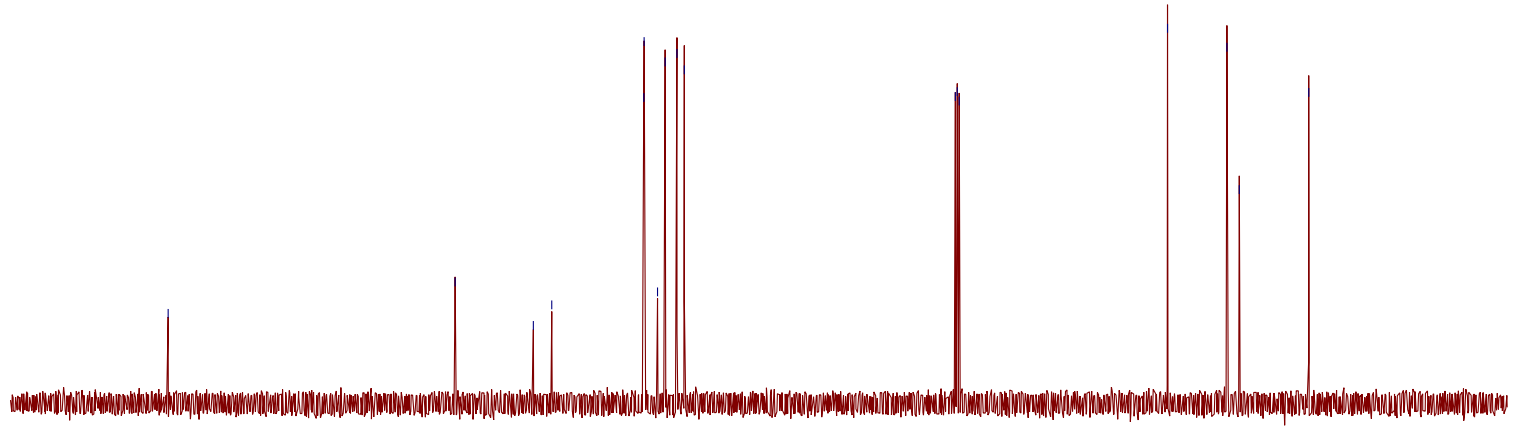

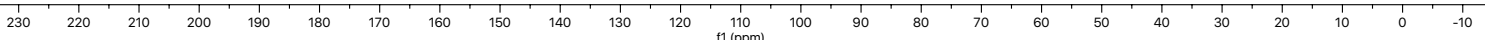




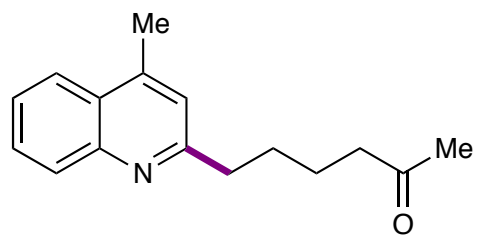

40

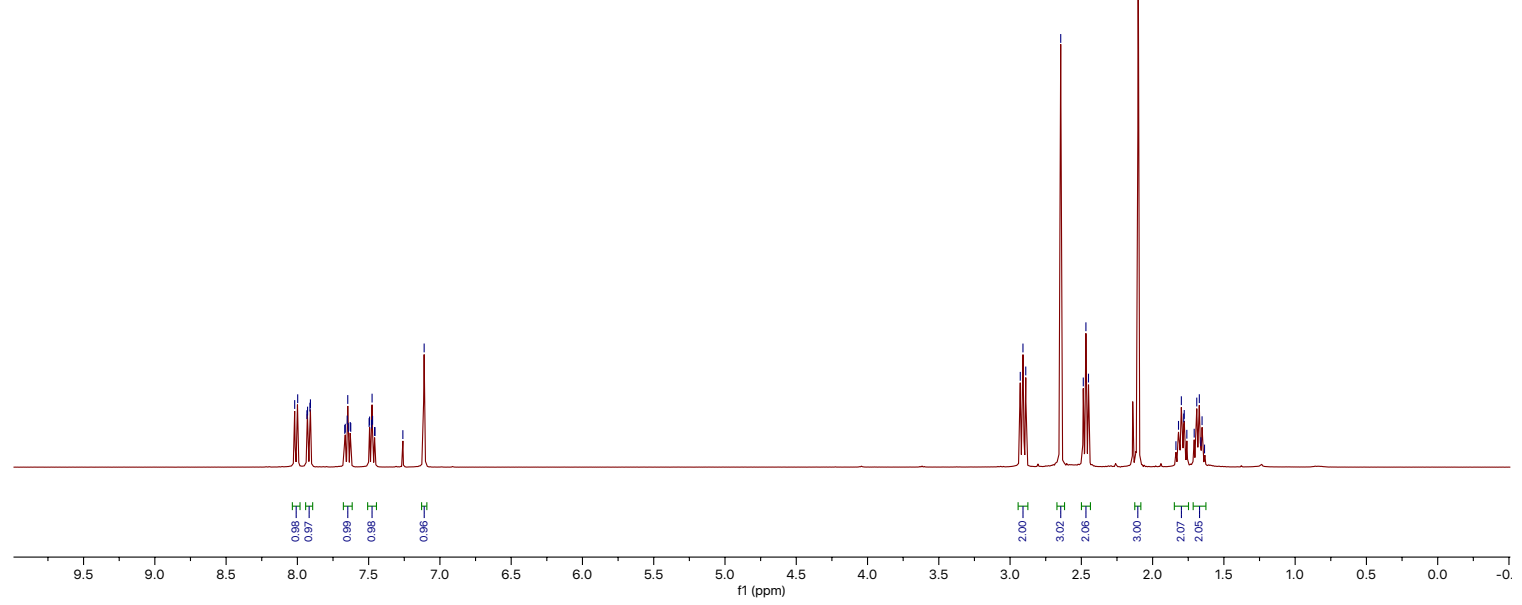

|z-95B1-1partt

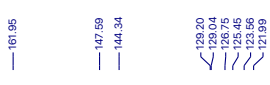

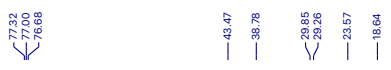<smiles>CC(=O)CCCCc1cc(C)c2ccccc2n1</smiles>

40

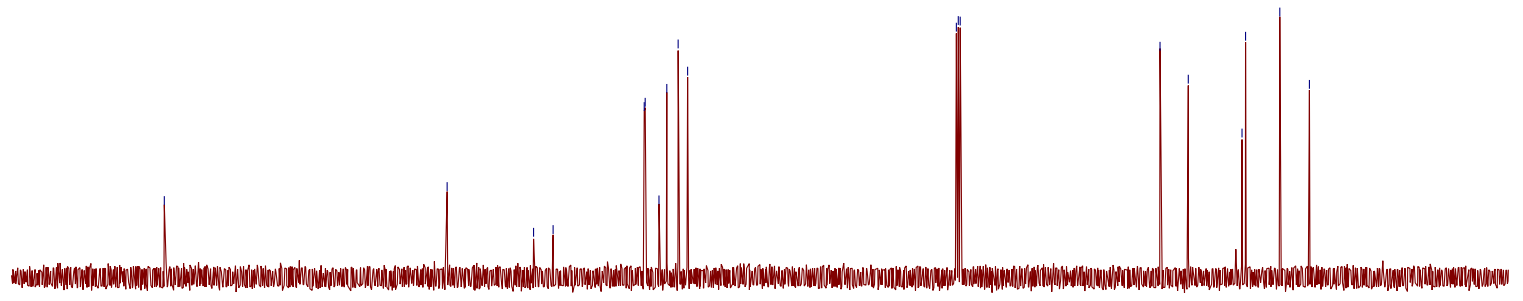

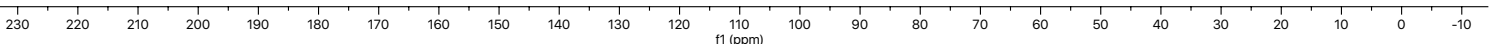


2011746-Iz-5-26p.10.fid

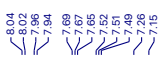

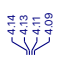

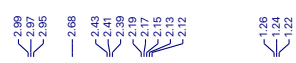<smiles>CCOC(=O)CCCc1cc(C)c2ccccc2n1</smiles>

41

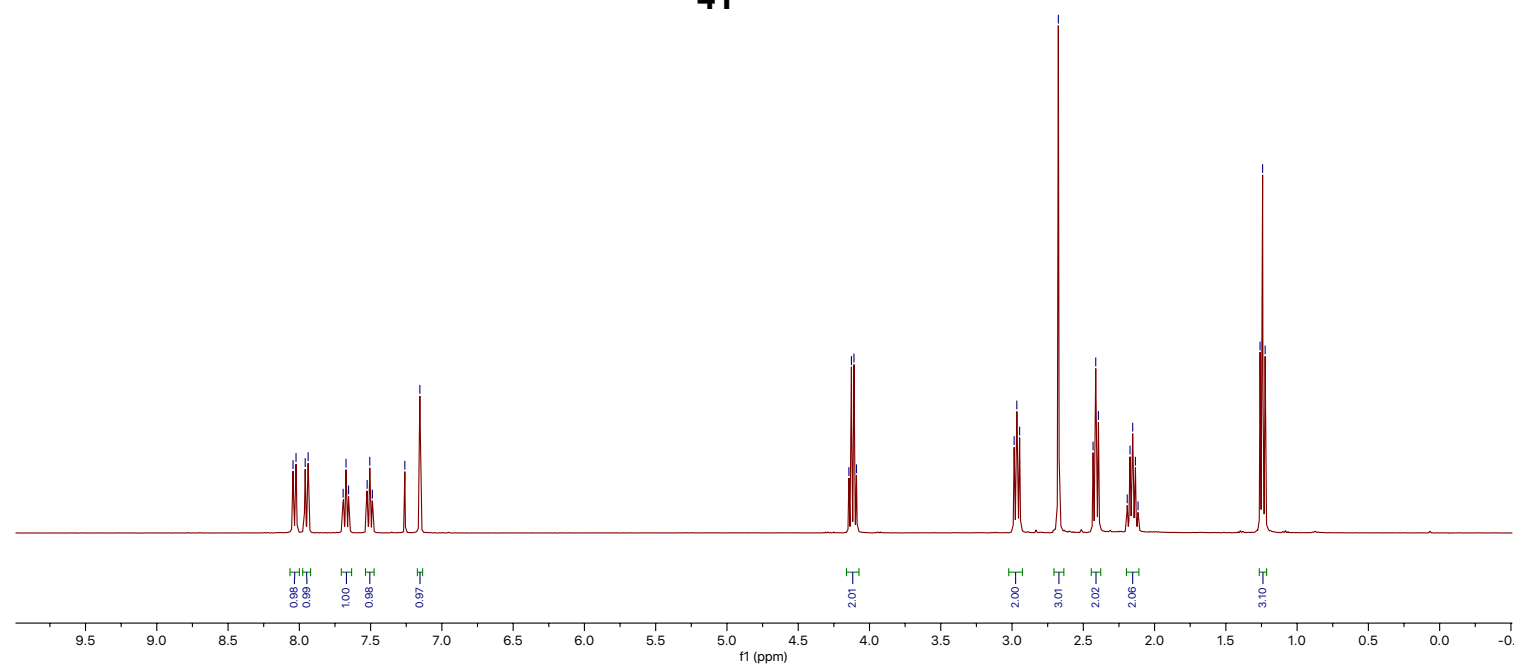

|z-4-13-P

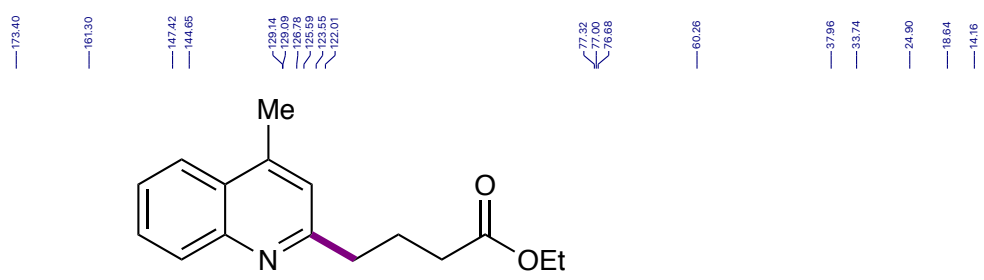

41

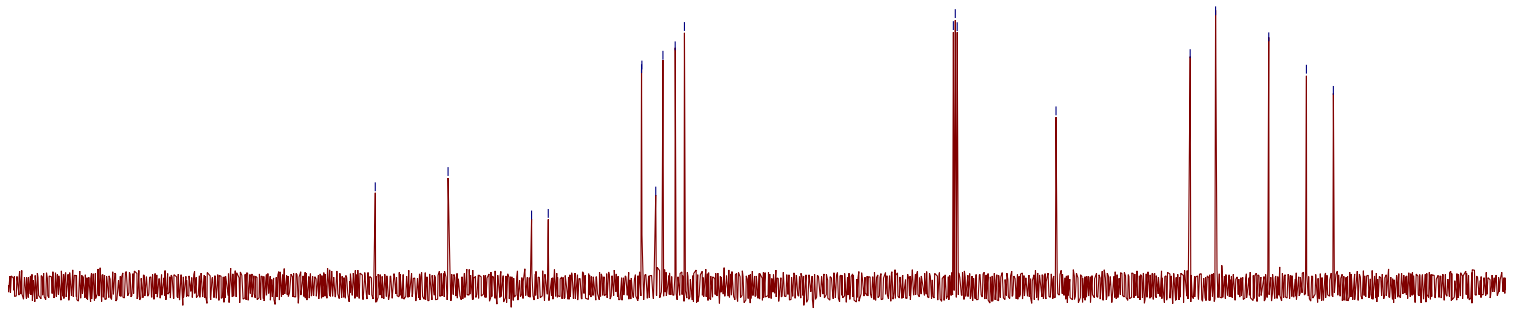

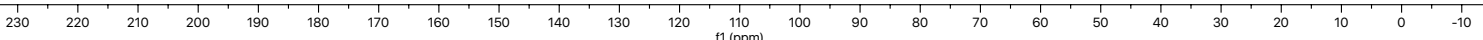


<smiles>Cc1cc(CCCC(F)(F)F)nc2ccccc12</smiles>

42

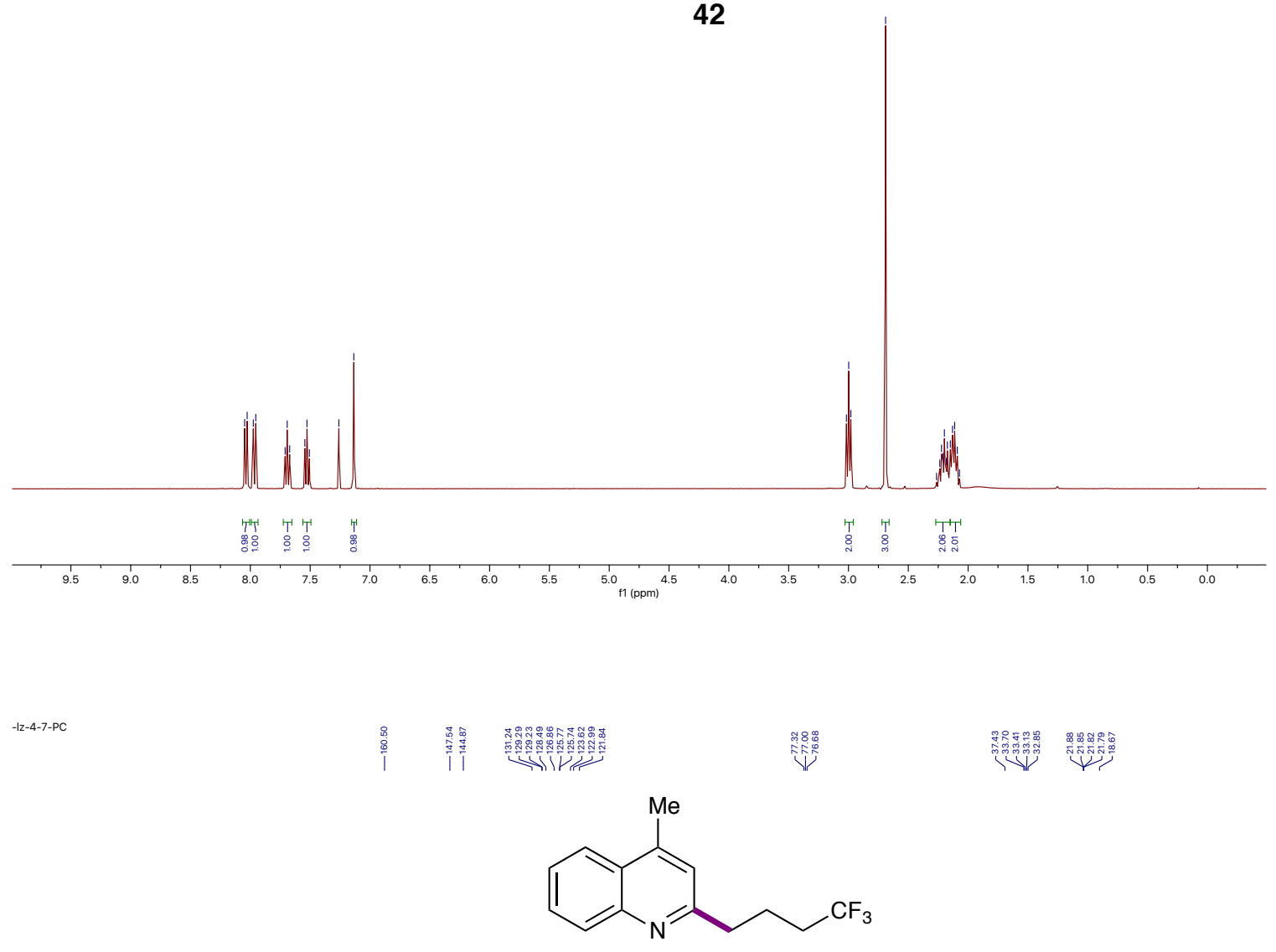

42

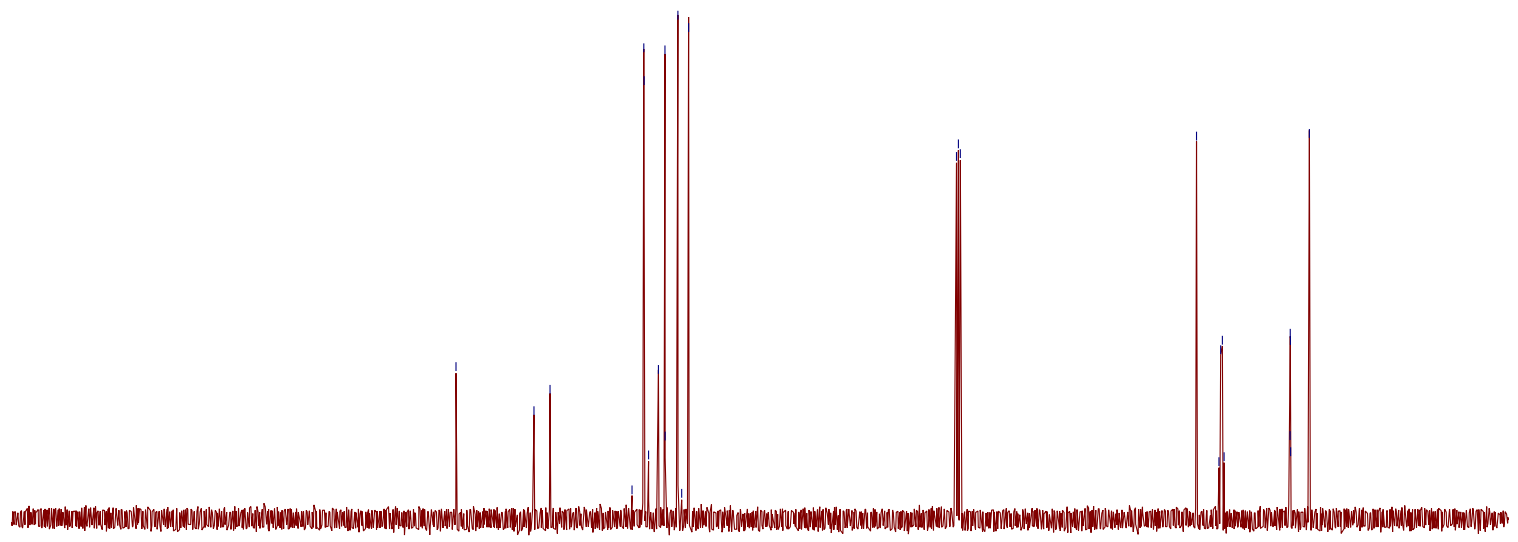

230

110
$\mathrm{f1}(\mathrm{ppm})$ 


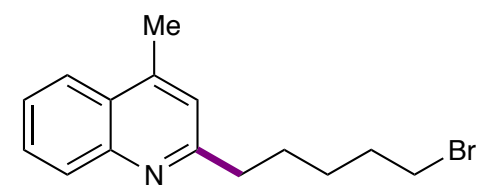

43

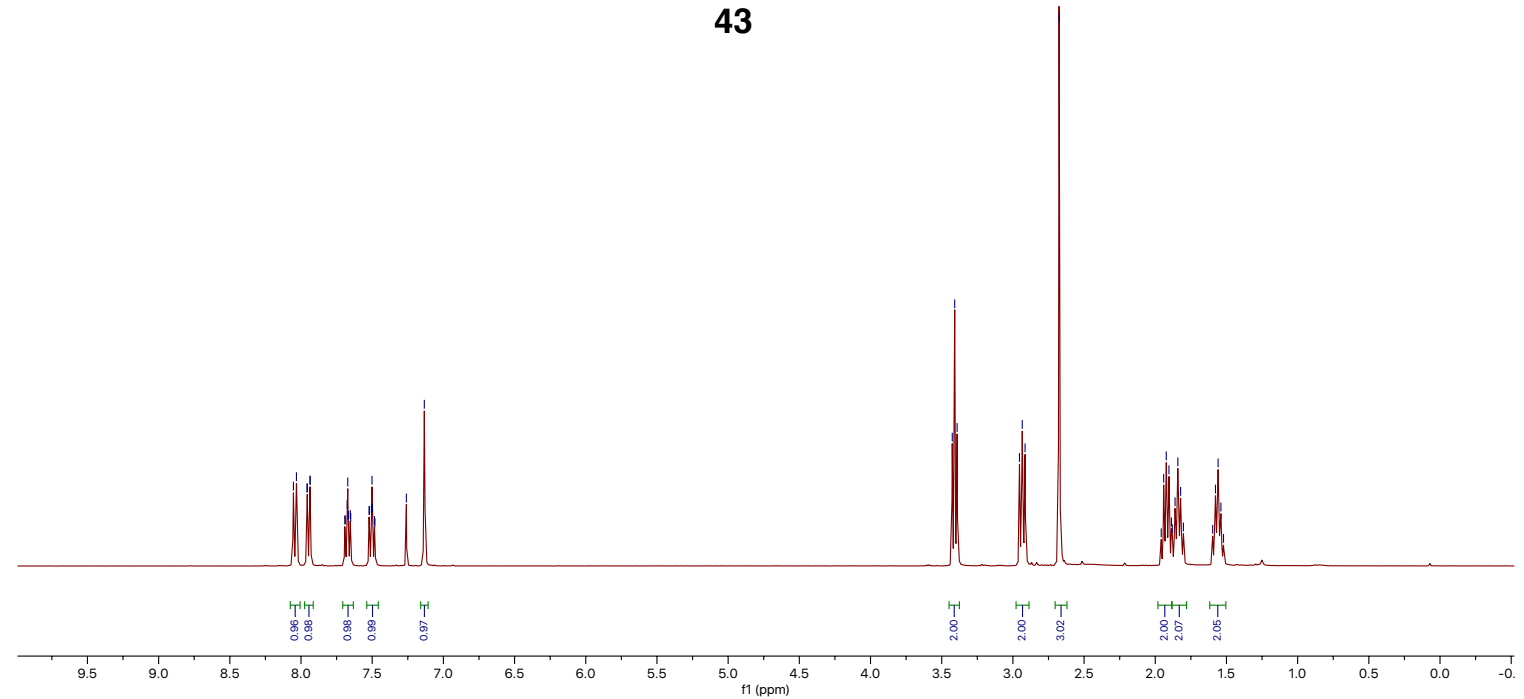

|z-4-8ap1
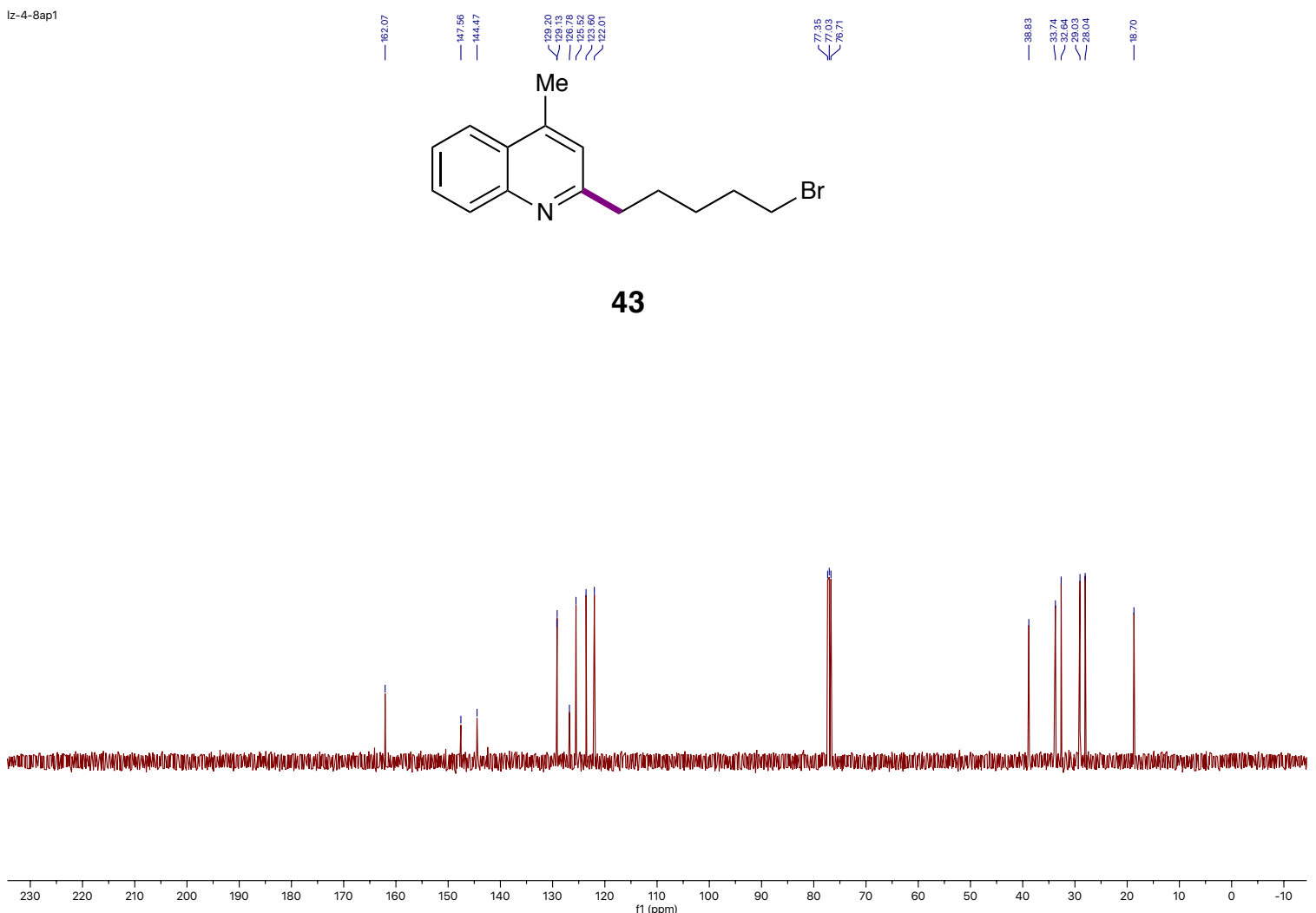
<smiles>Cc1cc(CC2CCOCC2)nc2ccccc12</smiles>

44

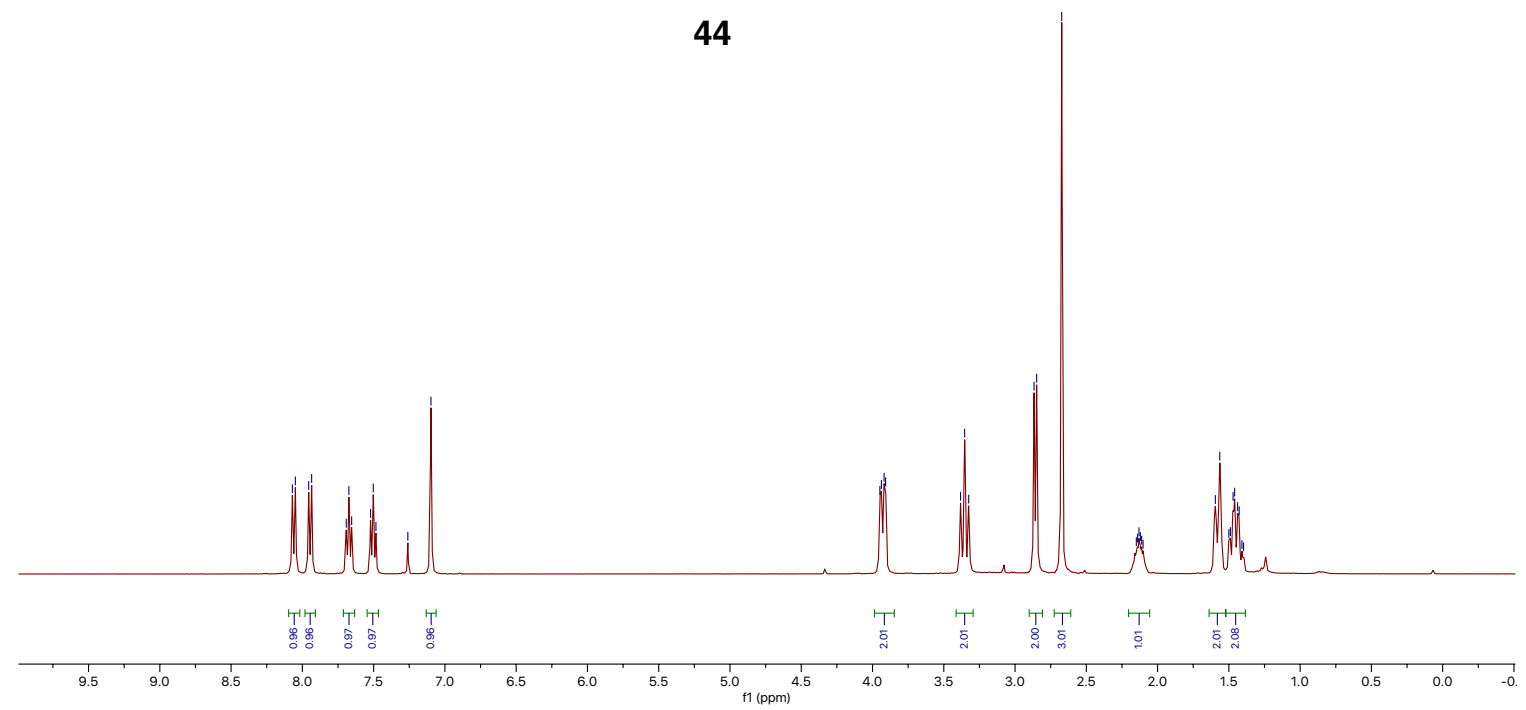

Iz-89E-2part

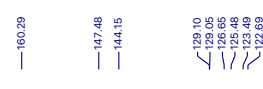

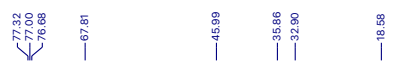<smiles>Cc1cc(CC2CCOCC2)nc2ccccc12</smiles>

44

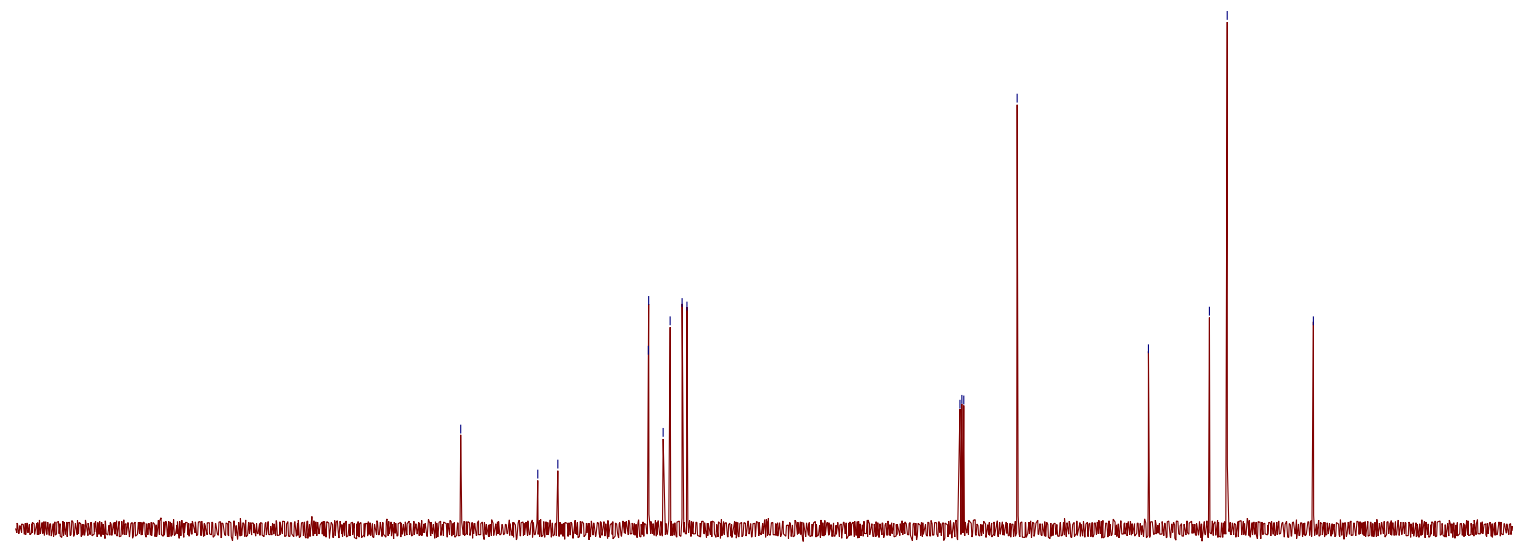

230 110
200 $\frac{1}{180 \quad 170}$ 
2011746-Iz-3-90CG.10.fid

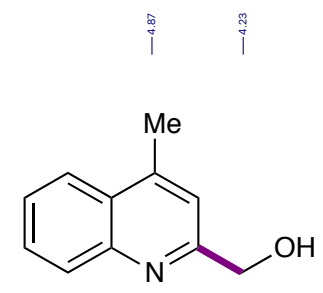

45

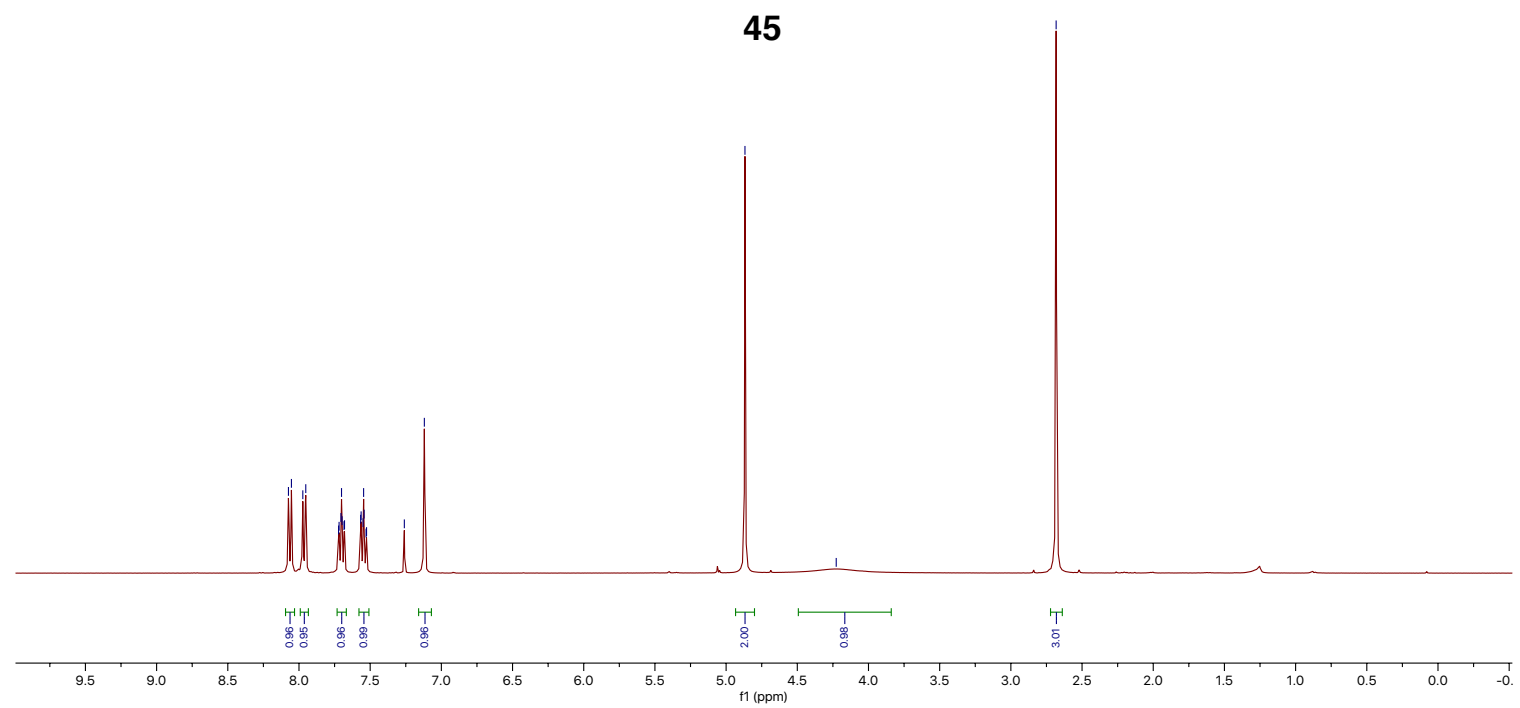

I-4-P8K

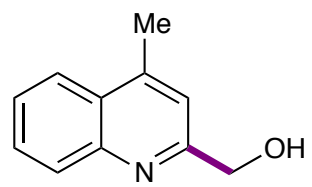

45

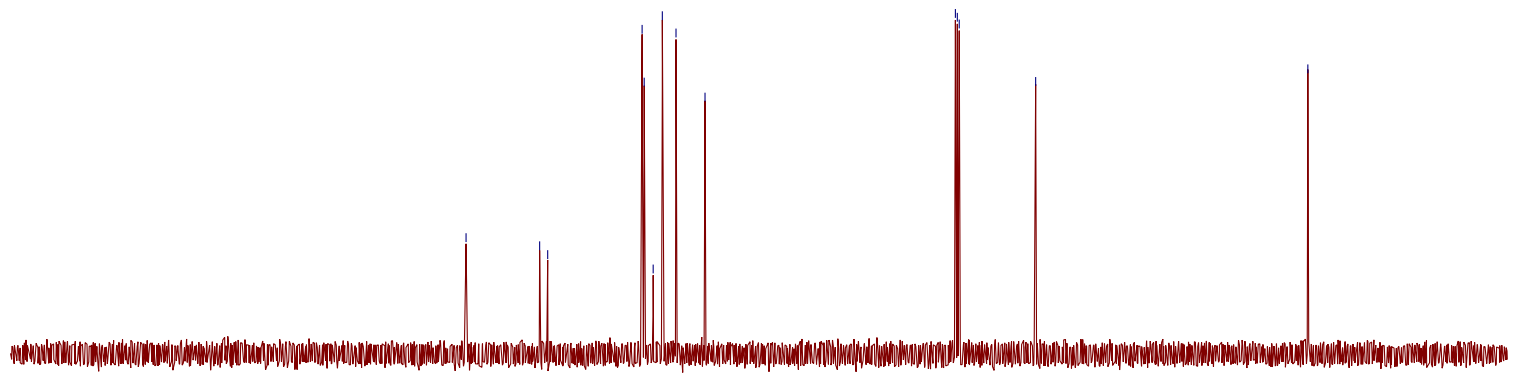

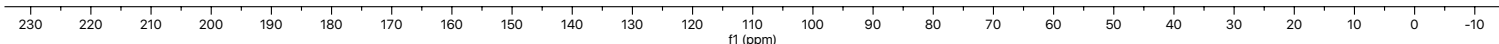



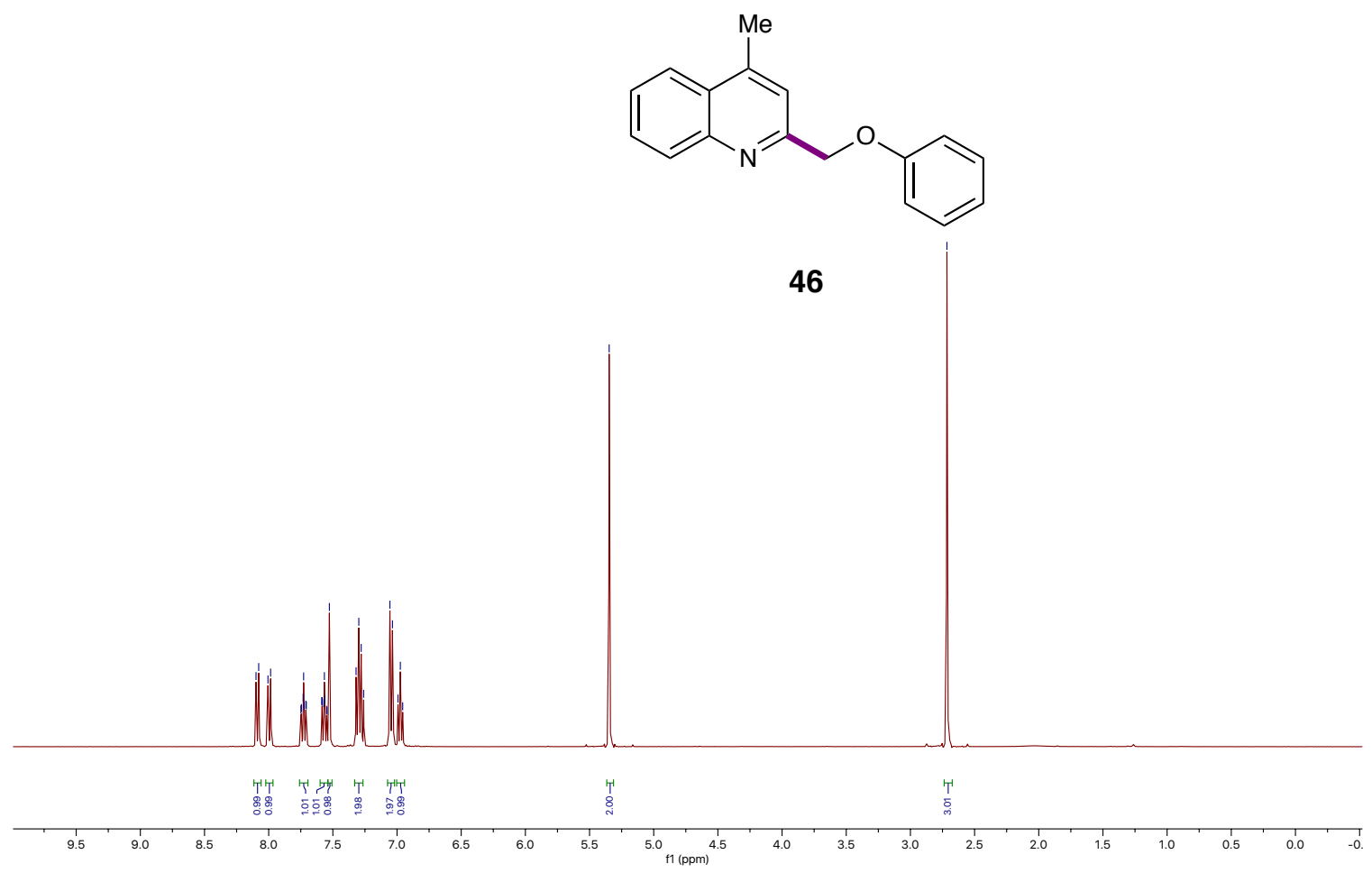

|z-5-38a1-1part

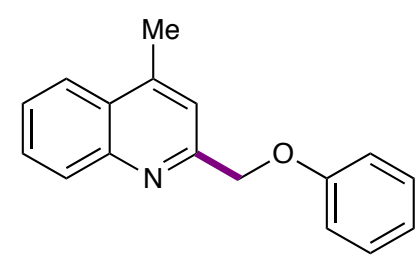

46

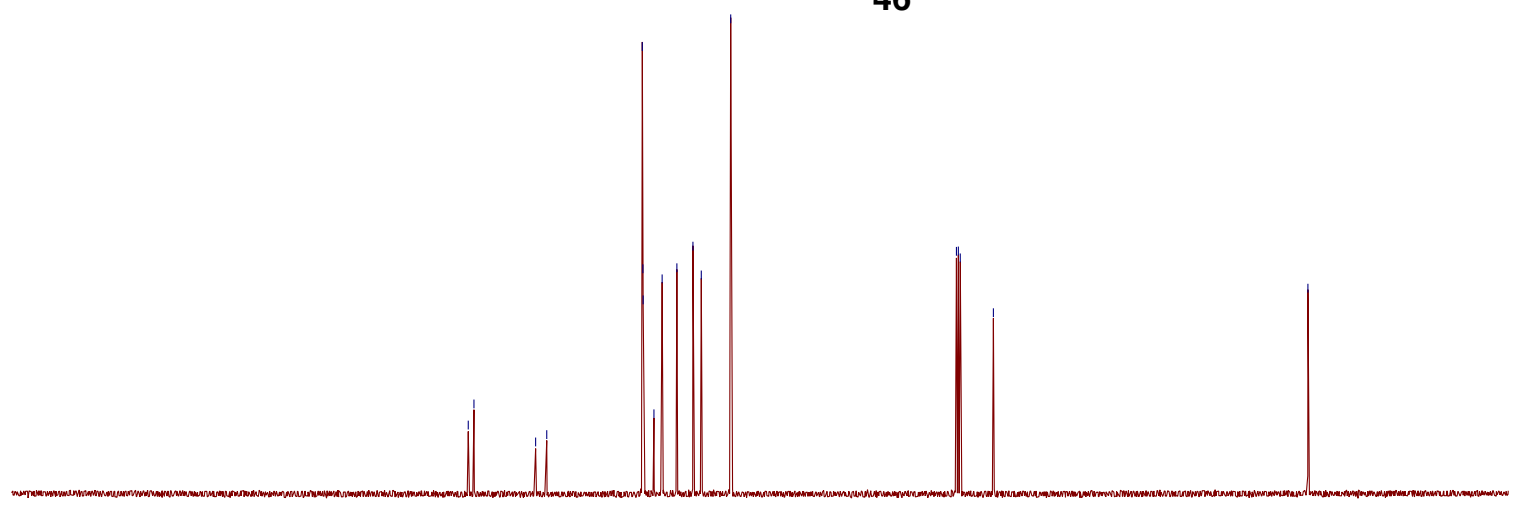

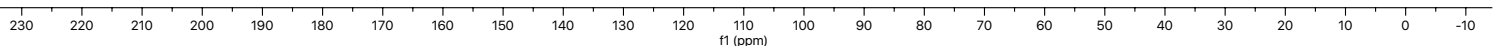




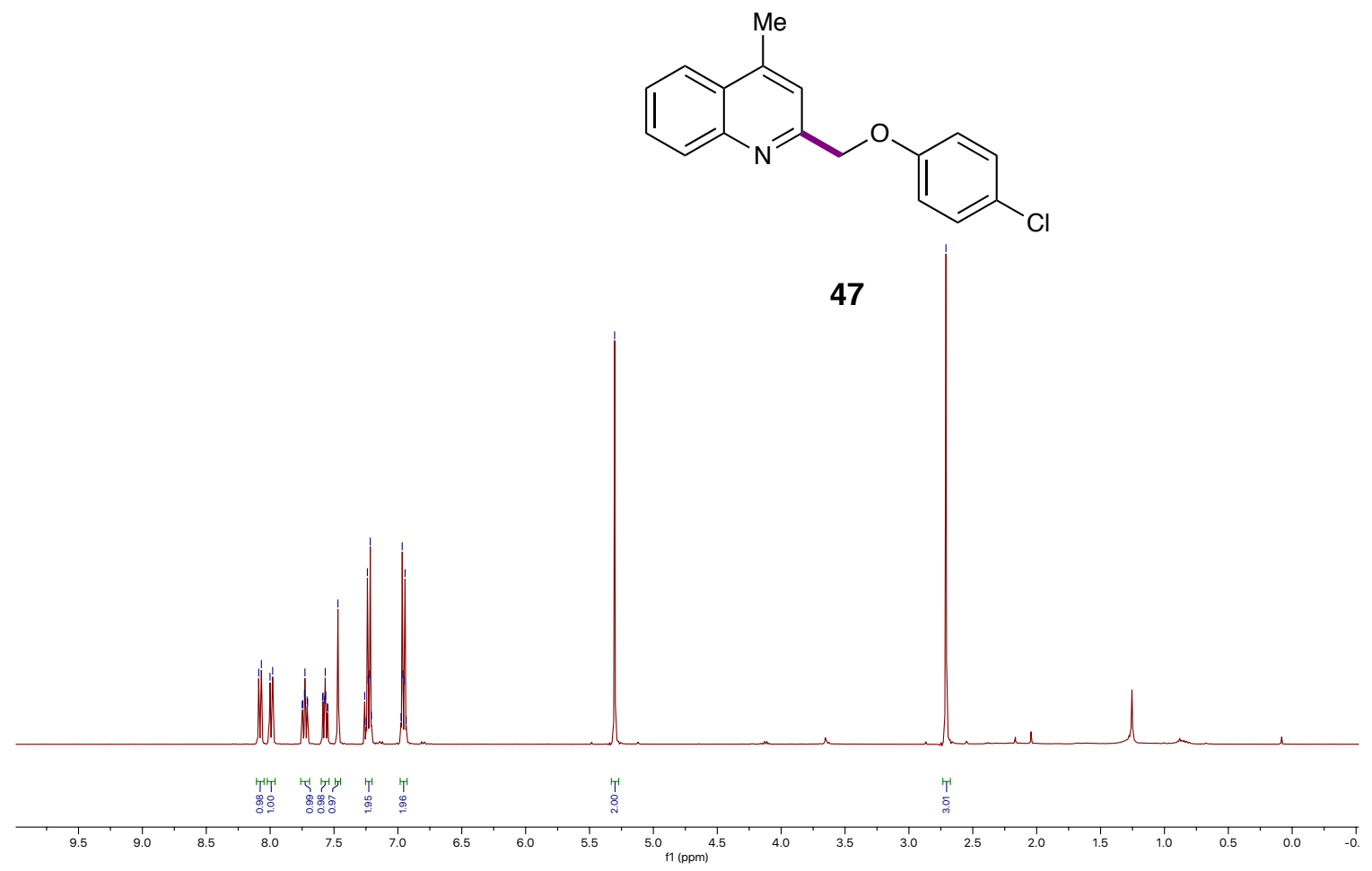

|z-5-41-3-part

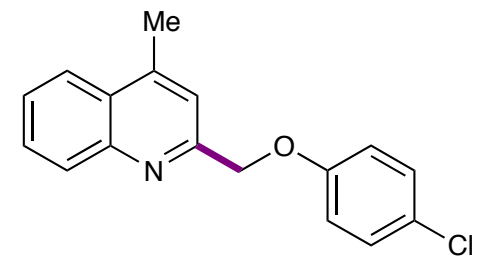

47

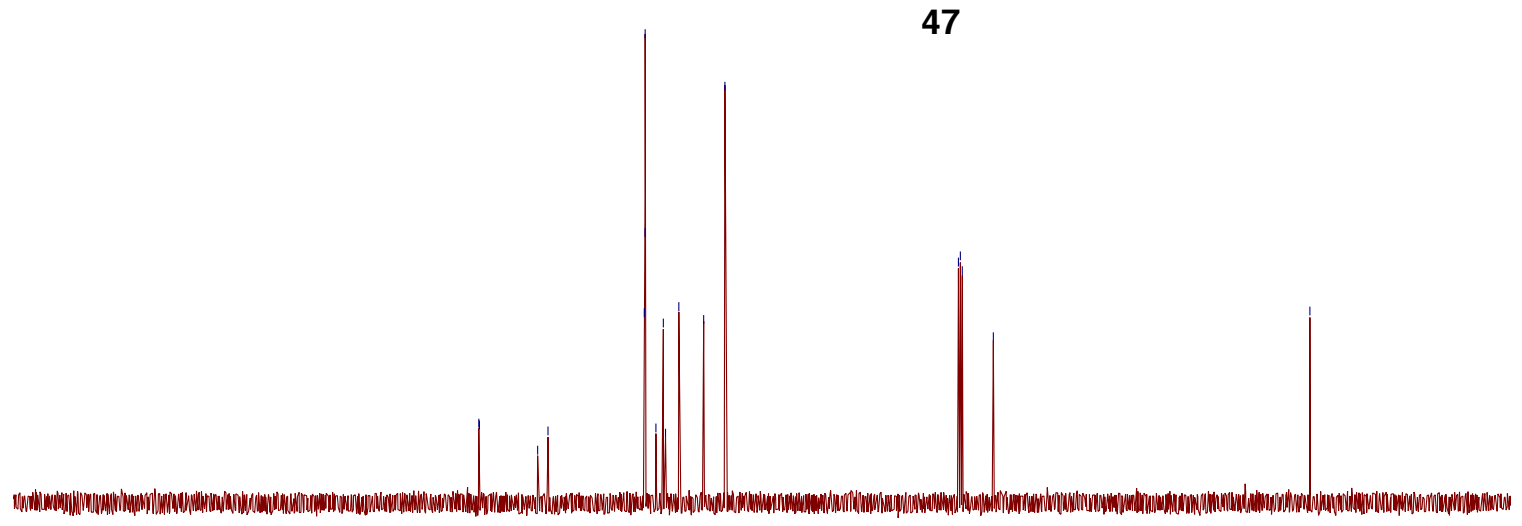

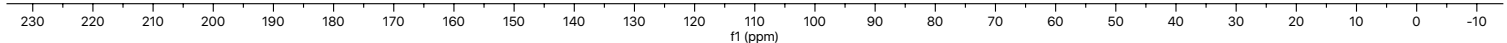


2011746-lz-4-6X-p. 10

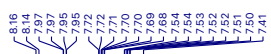

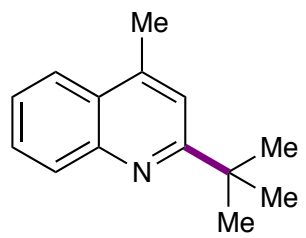

48

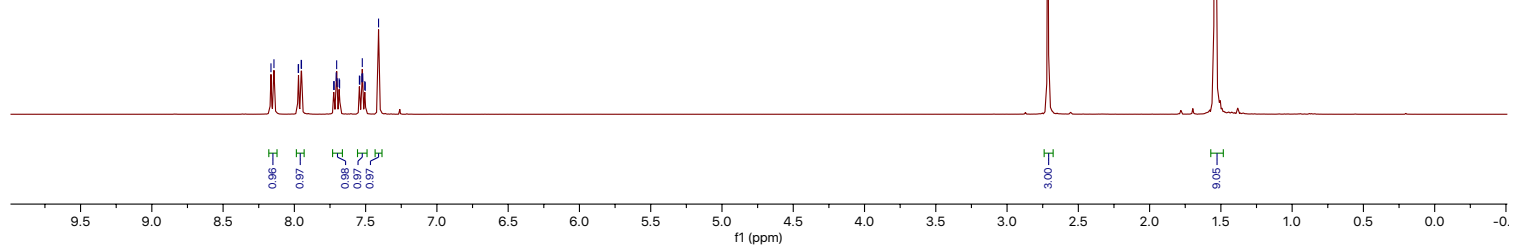

|z-6x-p

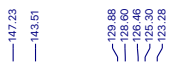
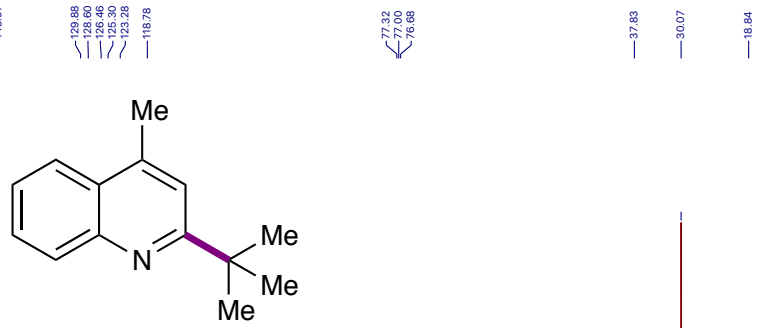

48
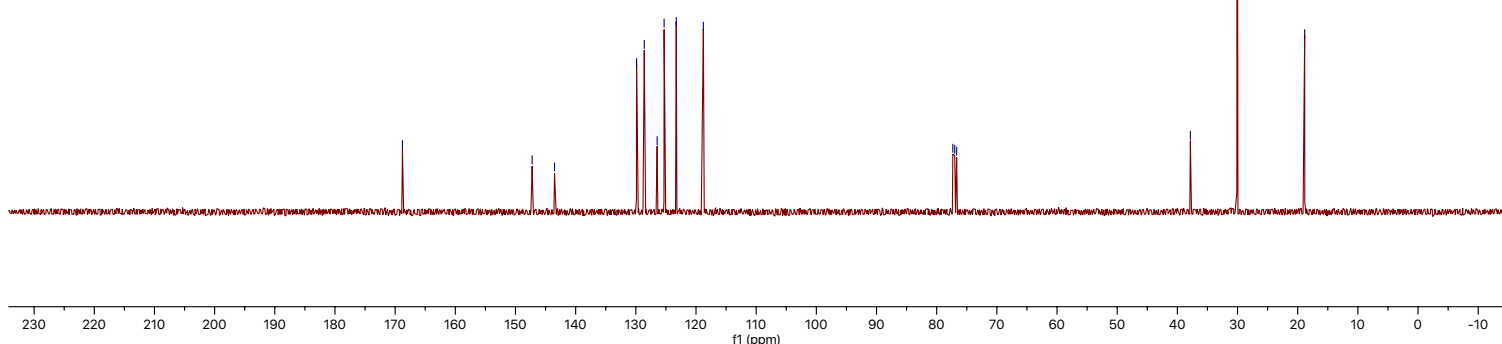

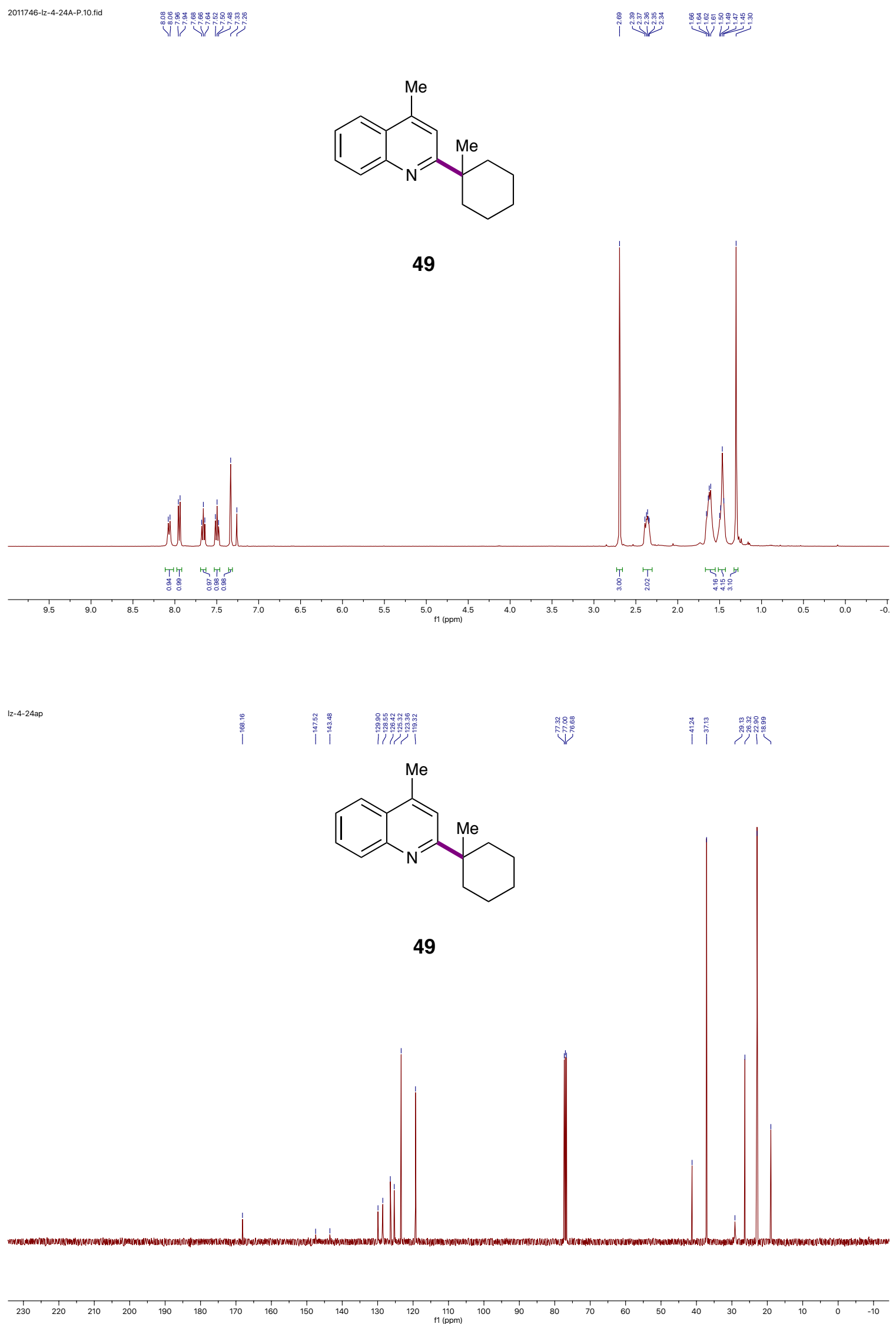
2011746-1z-5-17p-2.10.fid

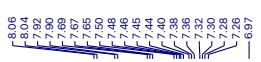

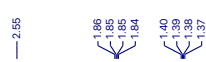<smiles>Cc1cc(C2(c3ccccc3)CC2)nc2ccccc12</smiles>

50

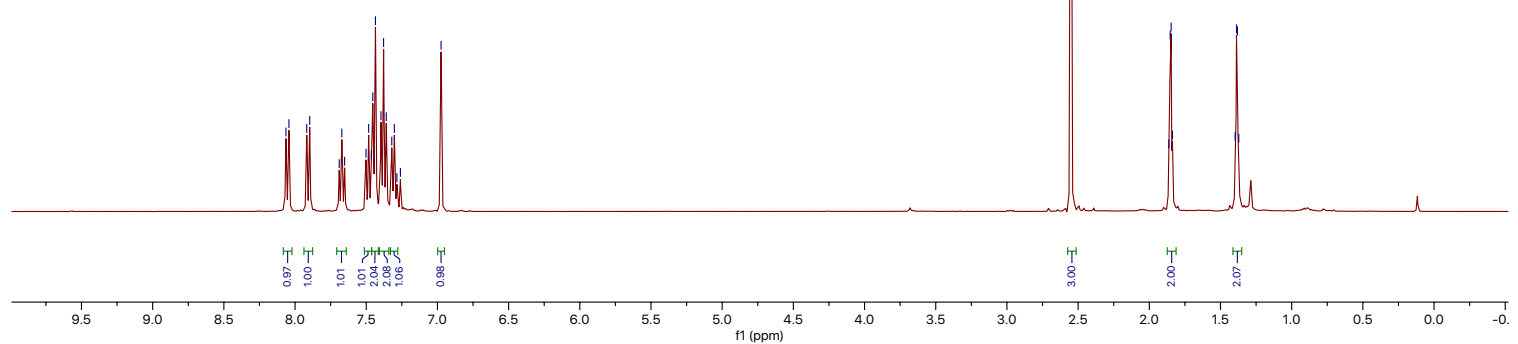

Iz-4-17ap
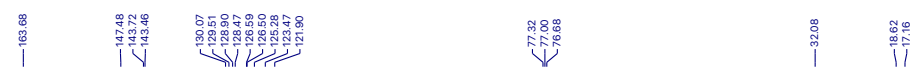<smiles>Cc1cc(C2(c3ccccc3)CC2)nc2ccccc12</smiles>

50

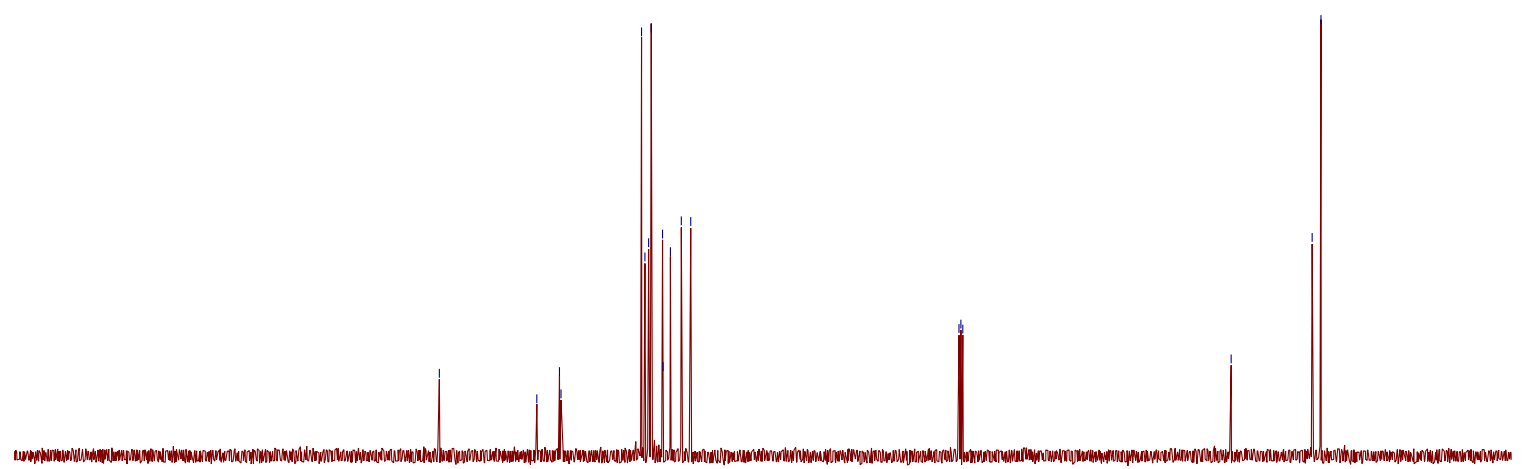

230

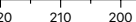

${ }_{190}^{1} \quad 18$

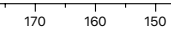

1

f1 1 (pp 
2011746-Iz-33B-P. 10.fid

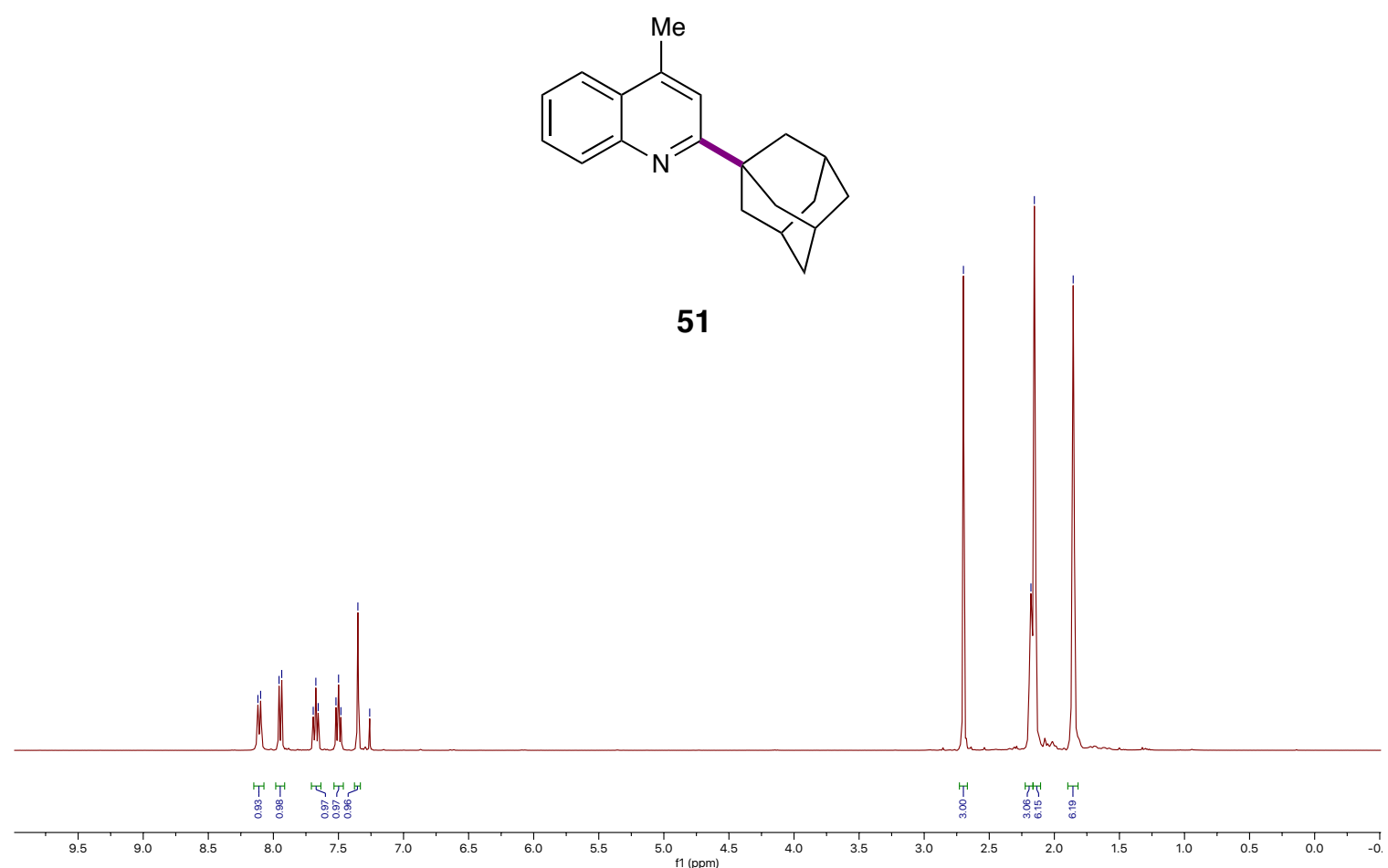

|z-4-33вр
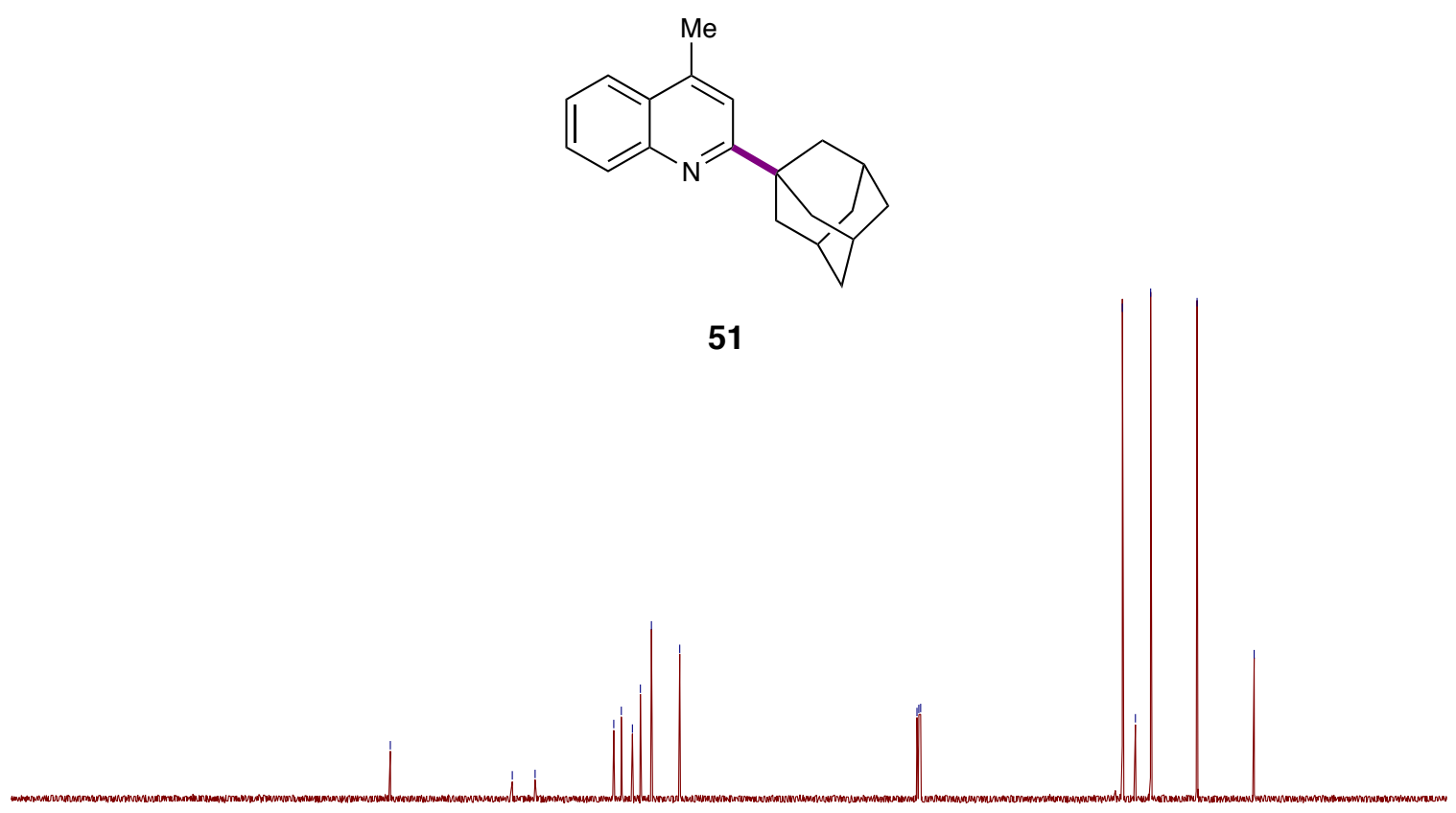

230

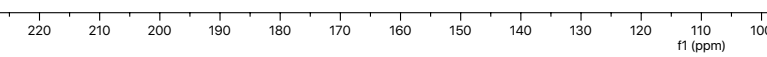




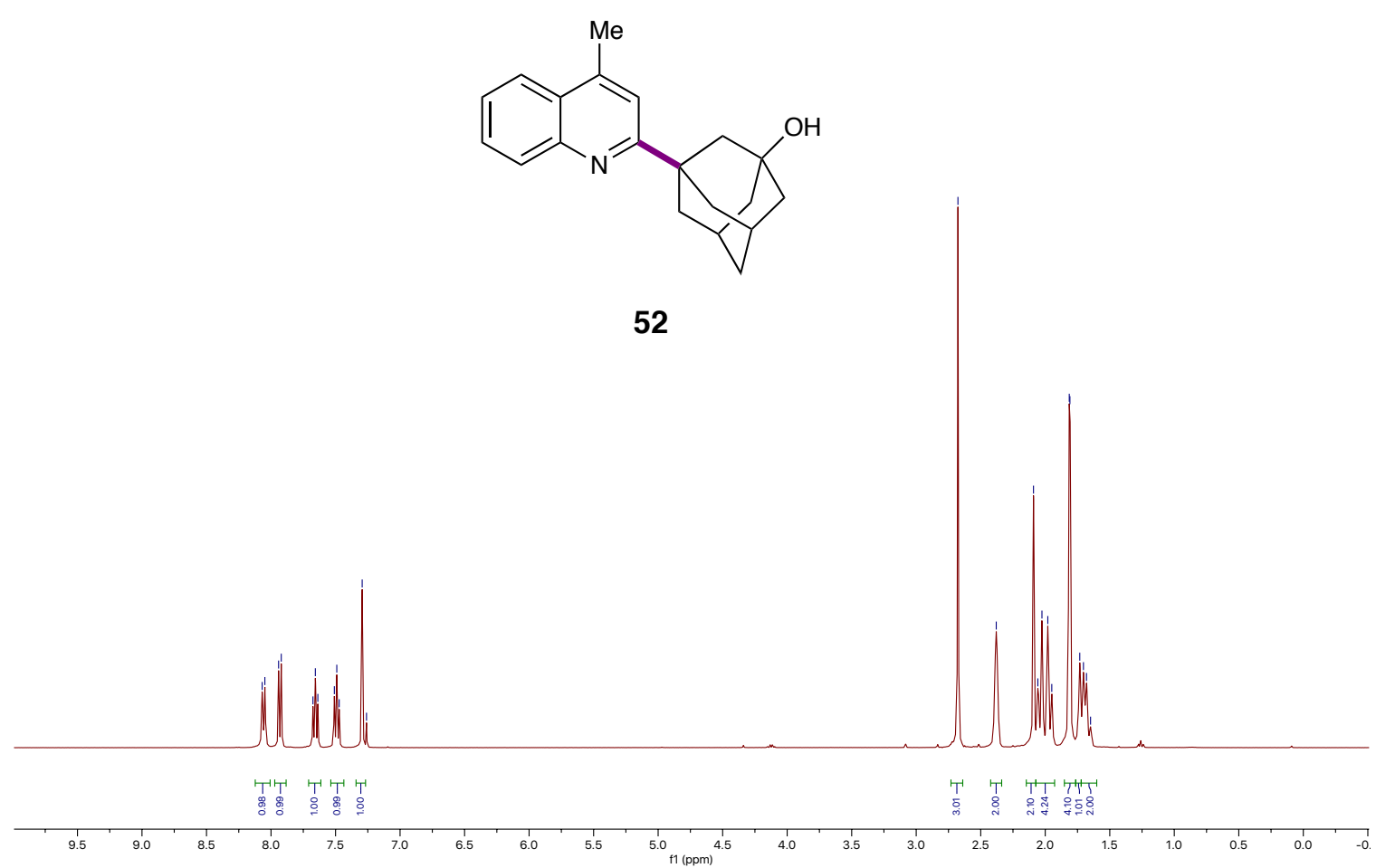

|z-90C-2part
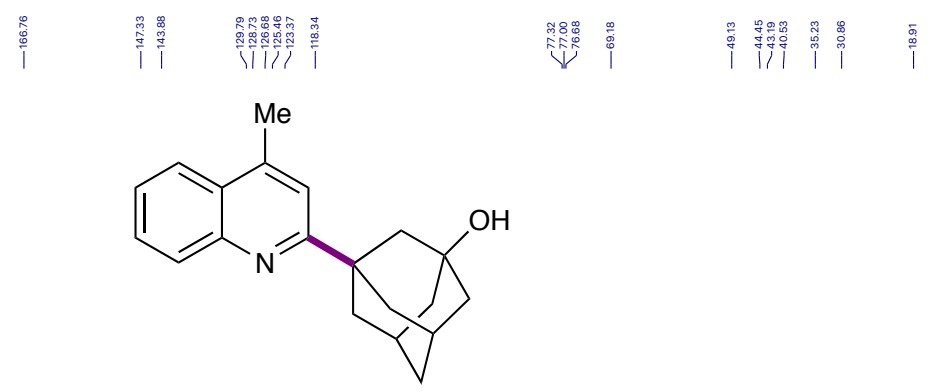

52

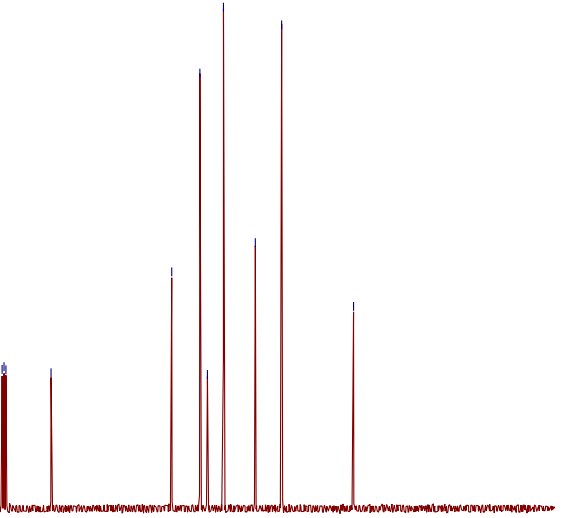

230

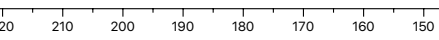

140

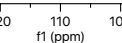




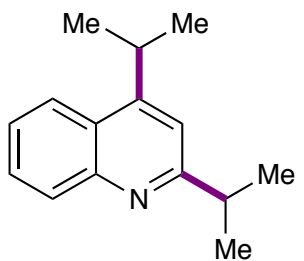

53

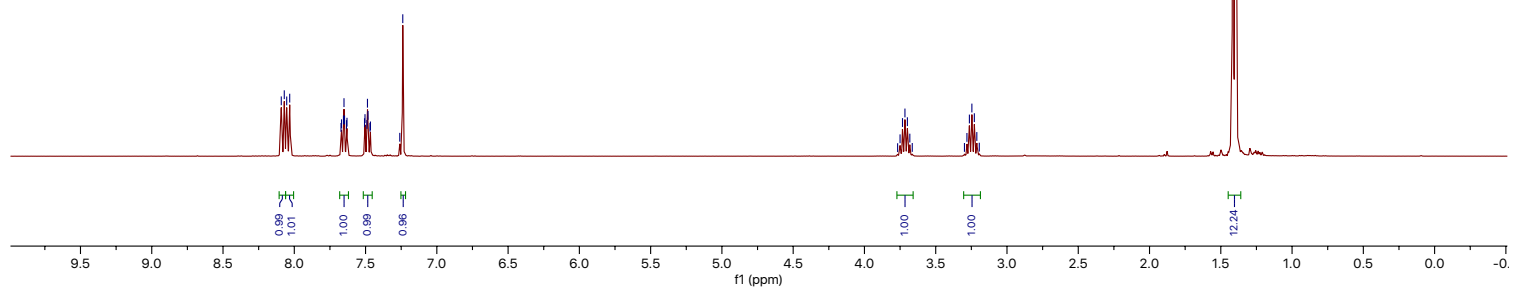

w-4-10|P

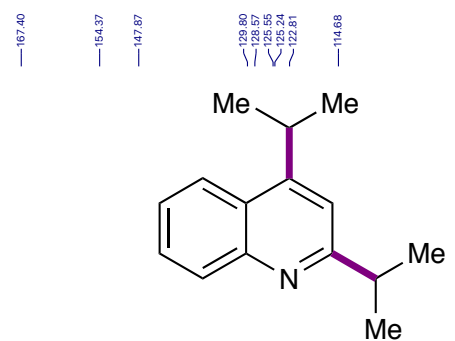

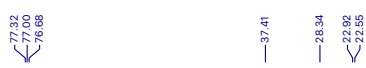

53

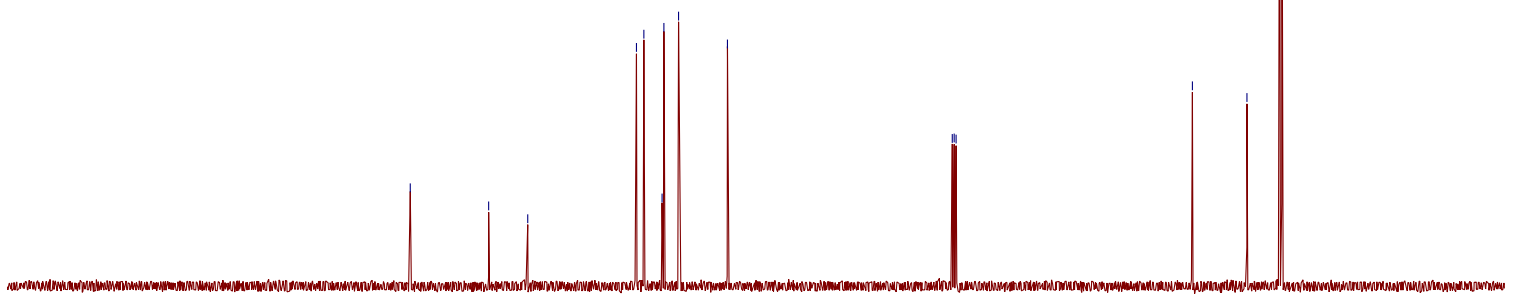




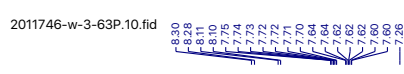

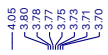

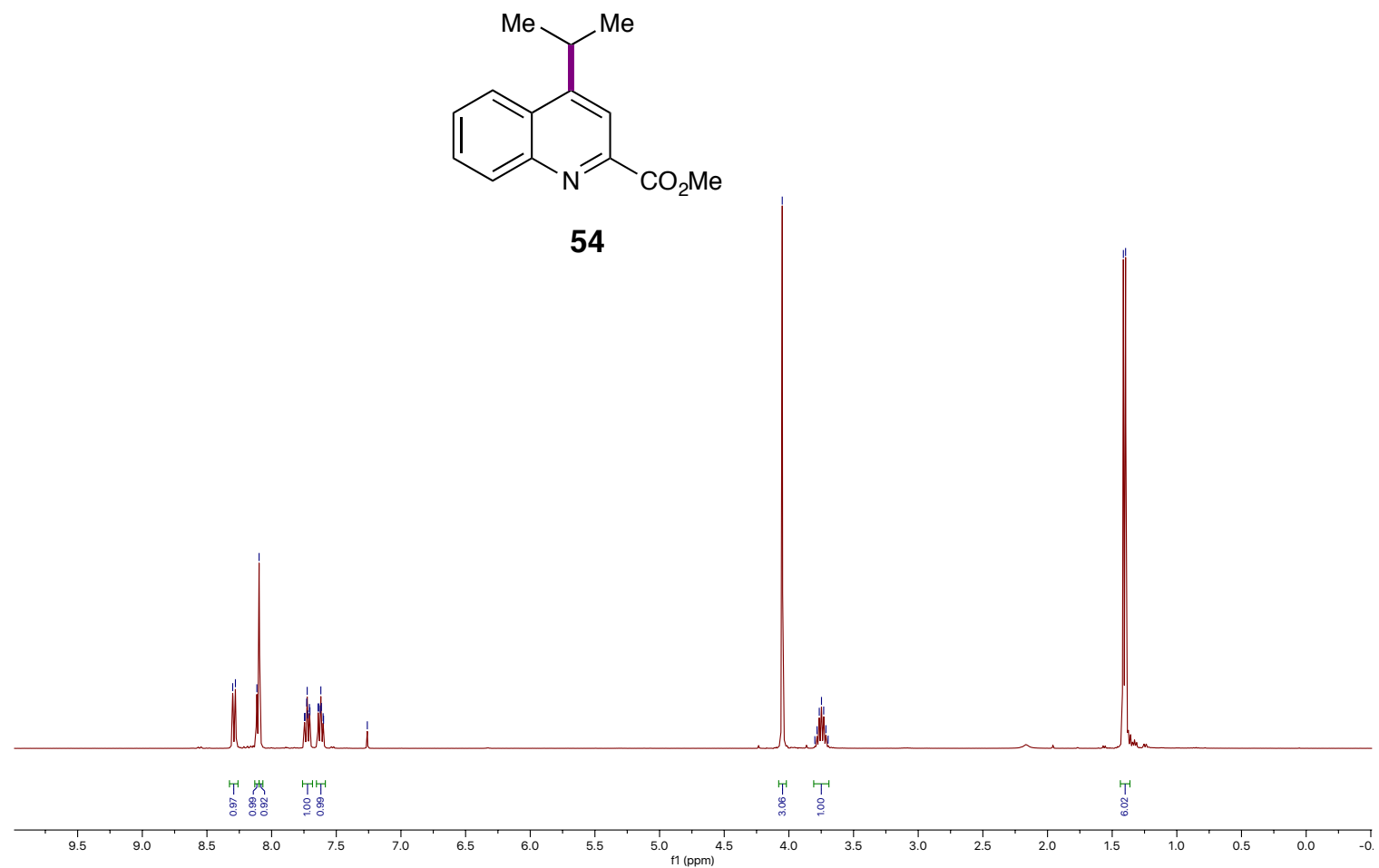

w-3-63-pC

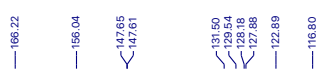

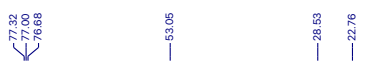

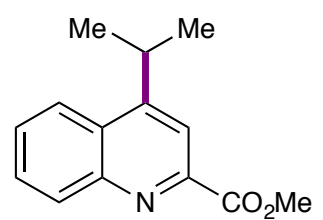

54

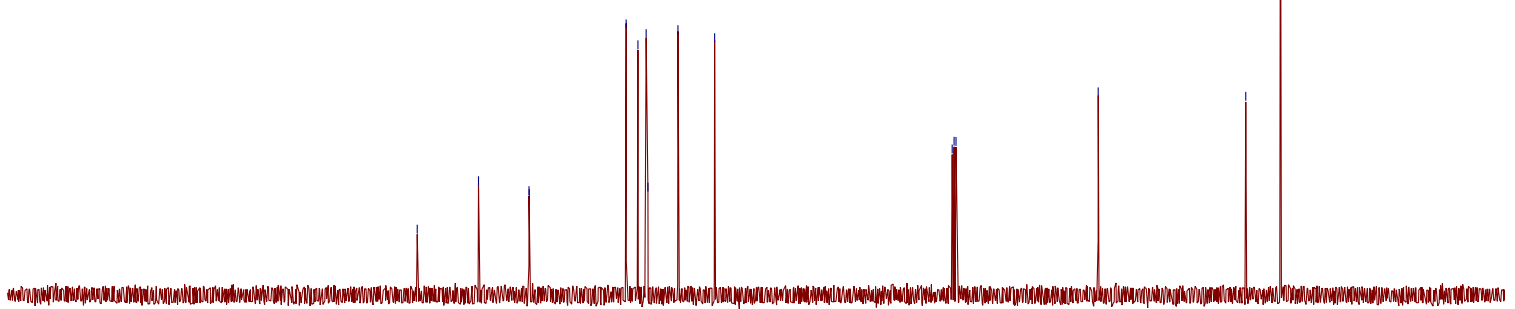

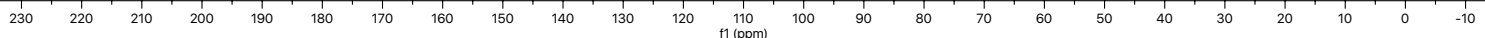



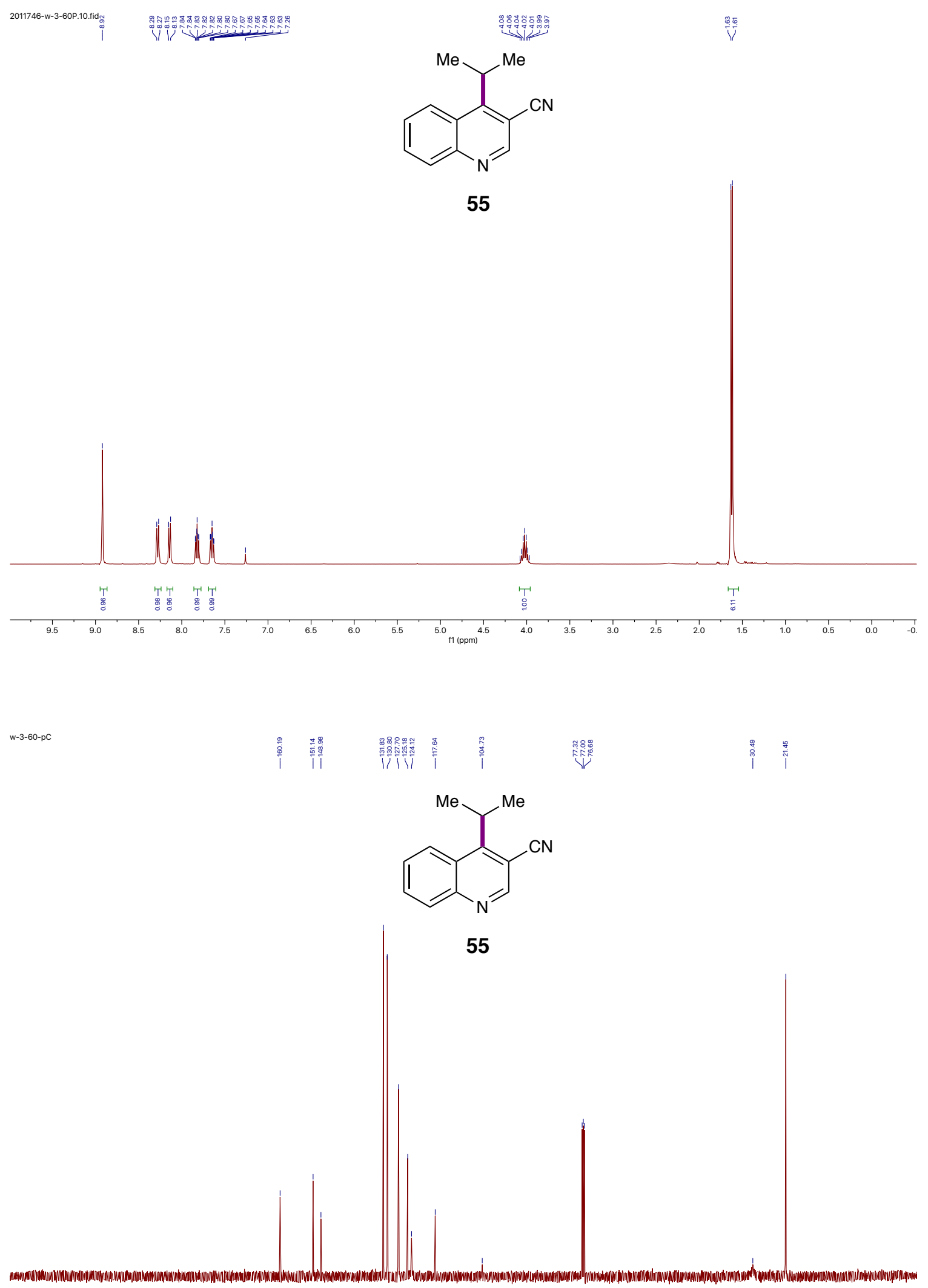

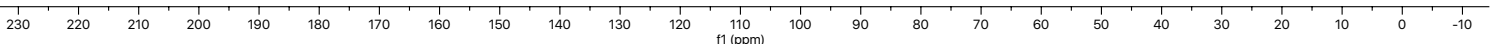


<smiles>CC(C)c1cc(Cl)c2ccccc2n1</smiles>

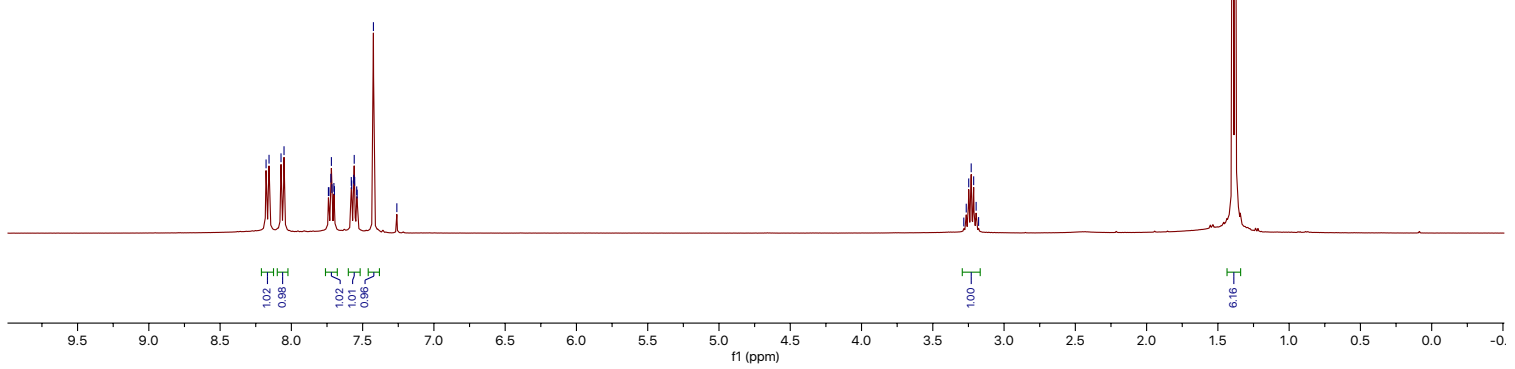

w-3-62-pC

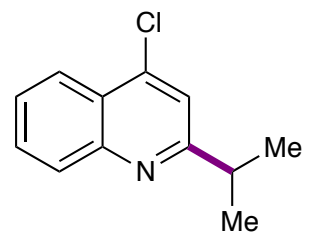

56

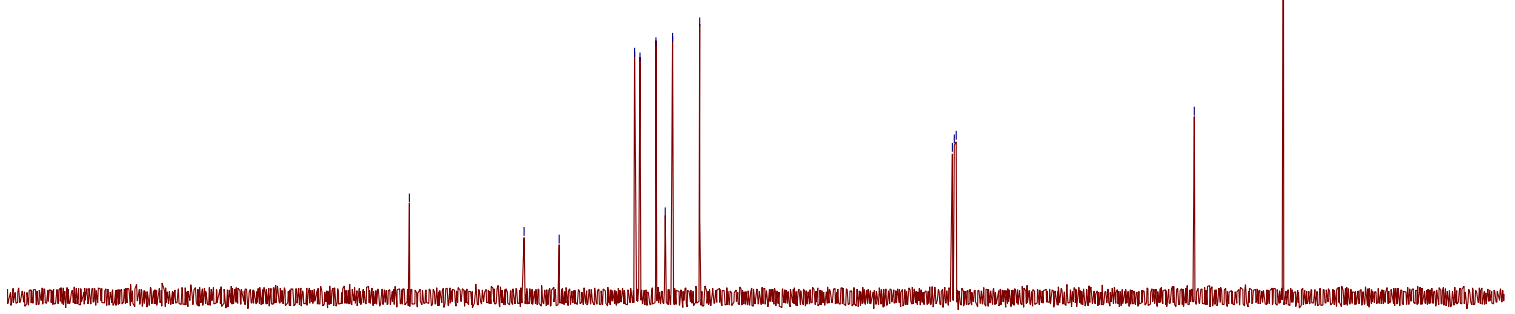

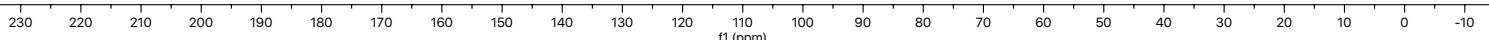




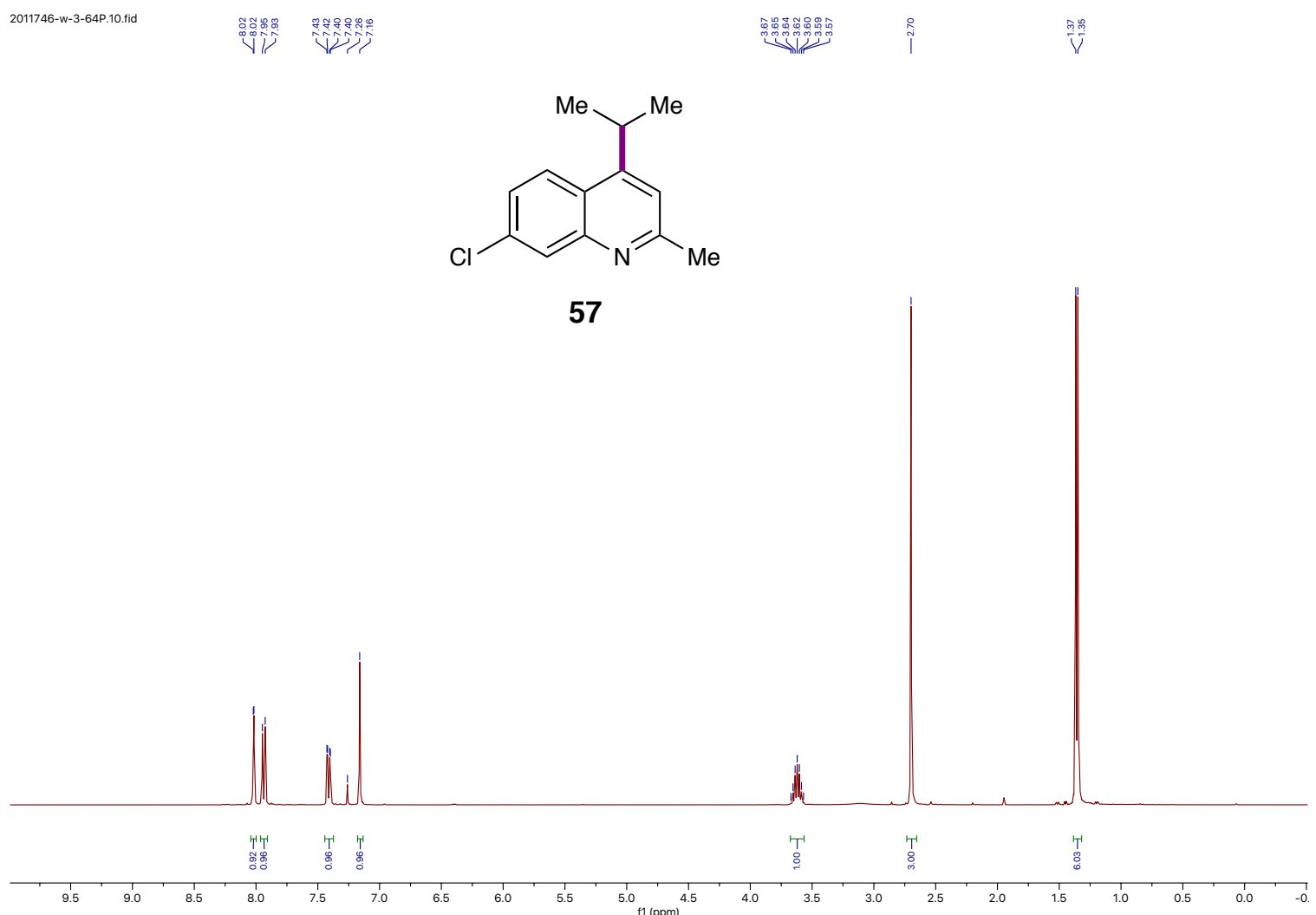

w-3-64-pC
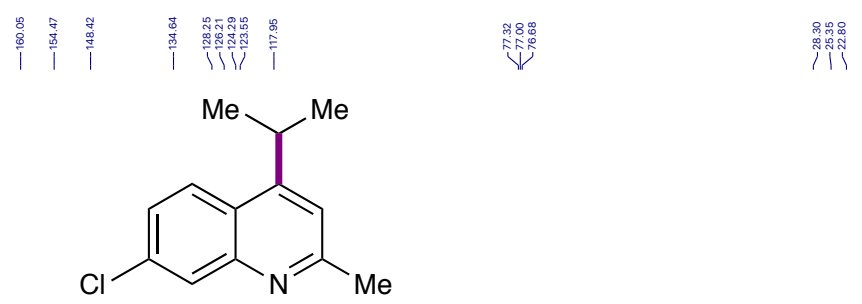

57

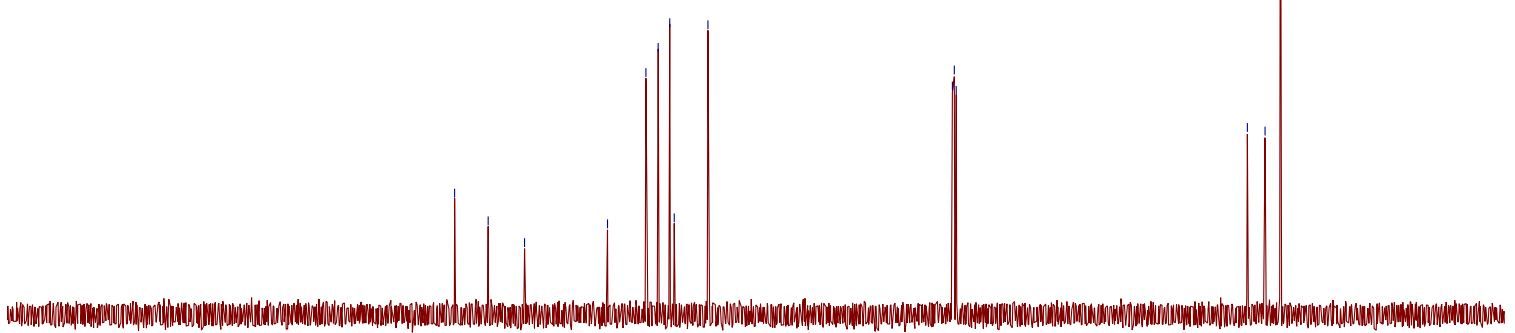

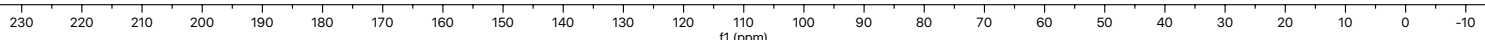



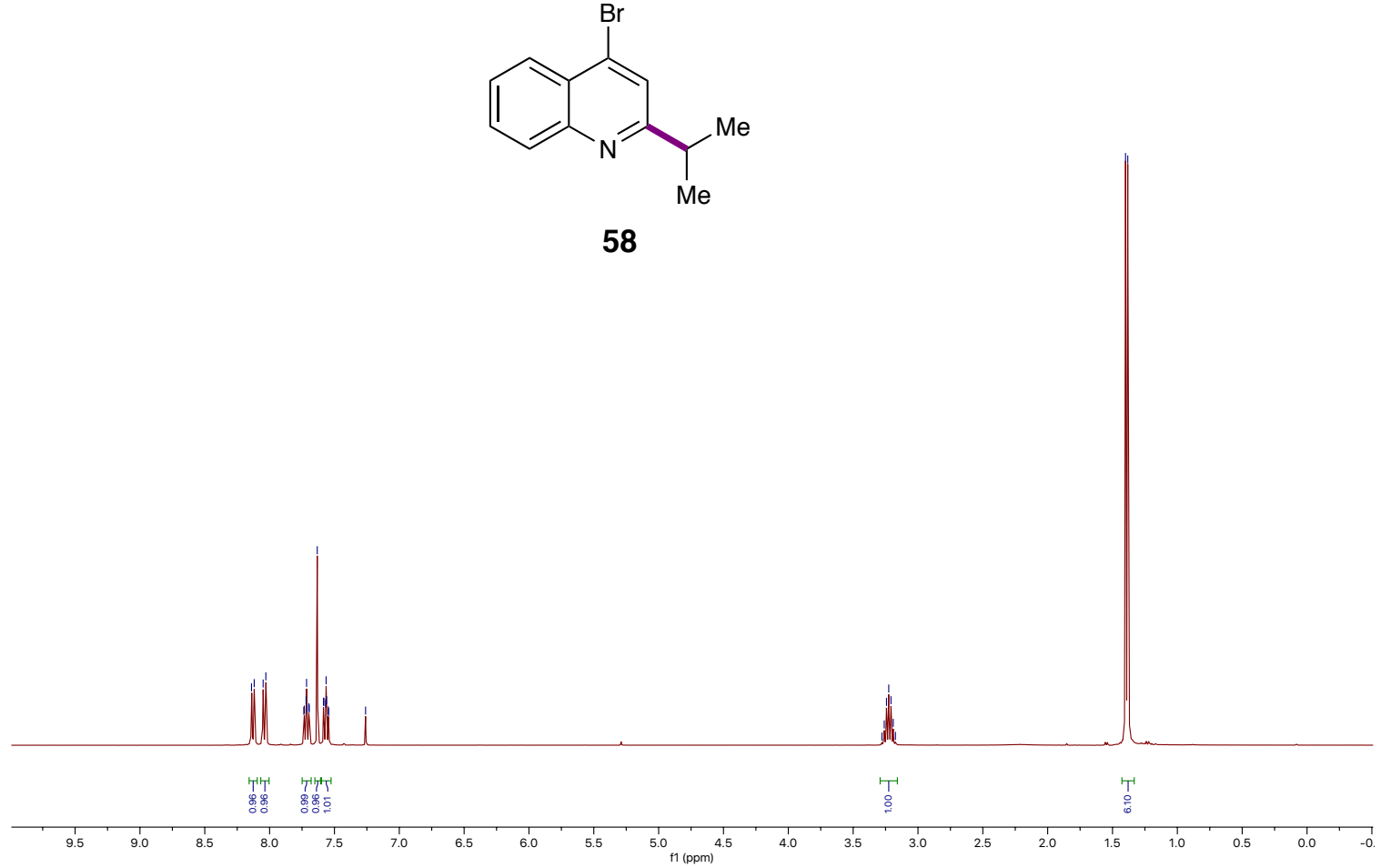

w-3-58p-C
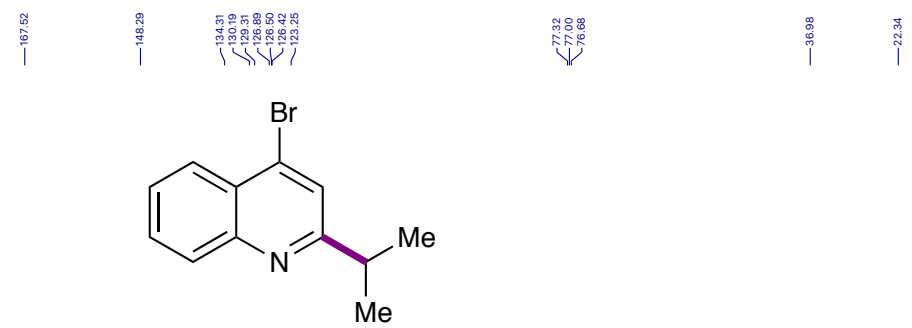

58

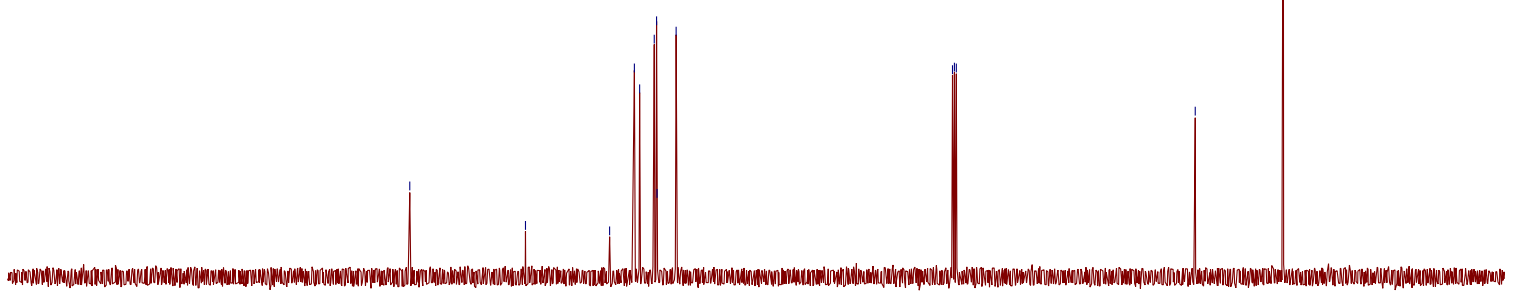

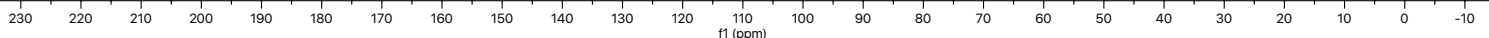




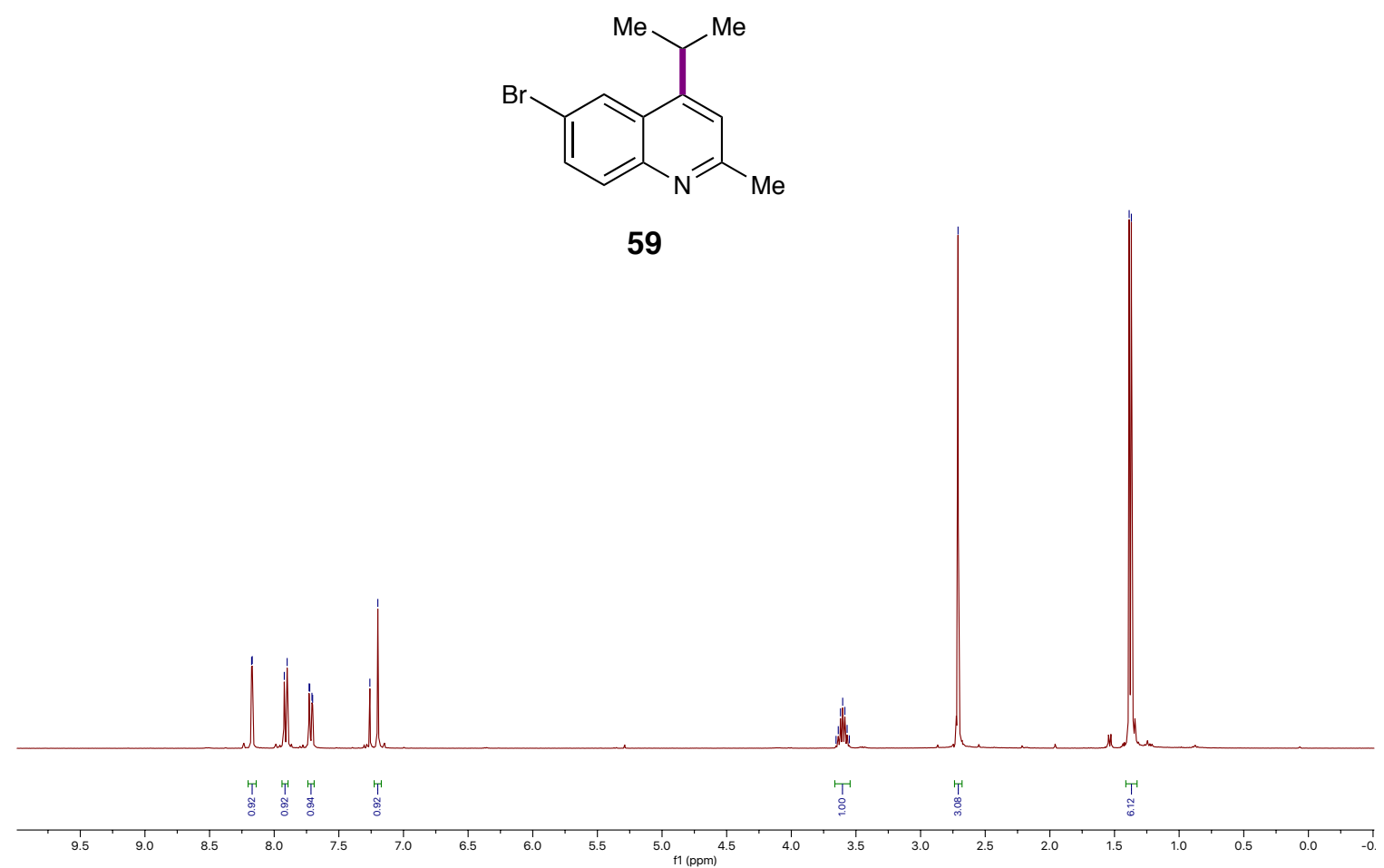

w-3-59-pC

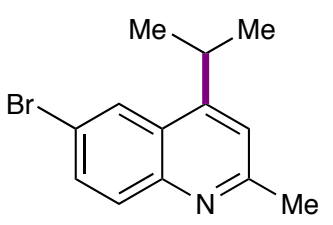

59

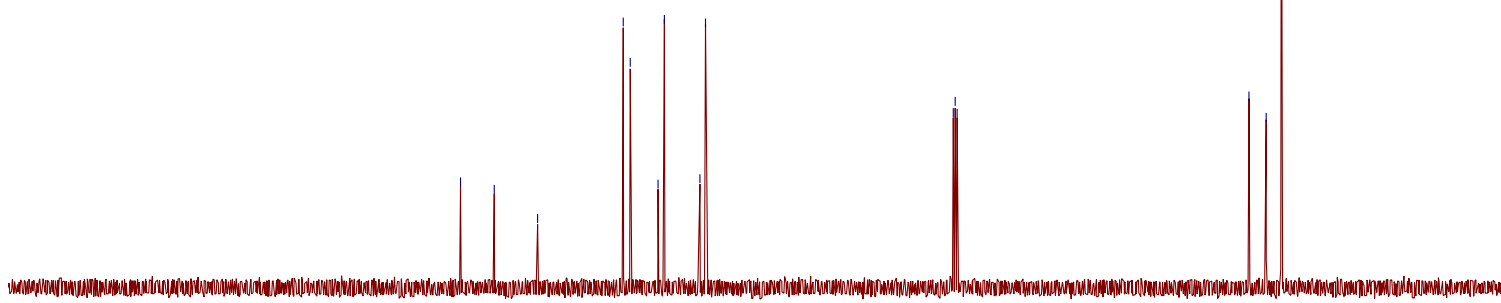



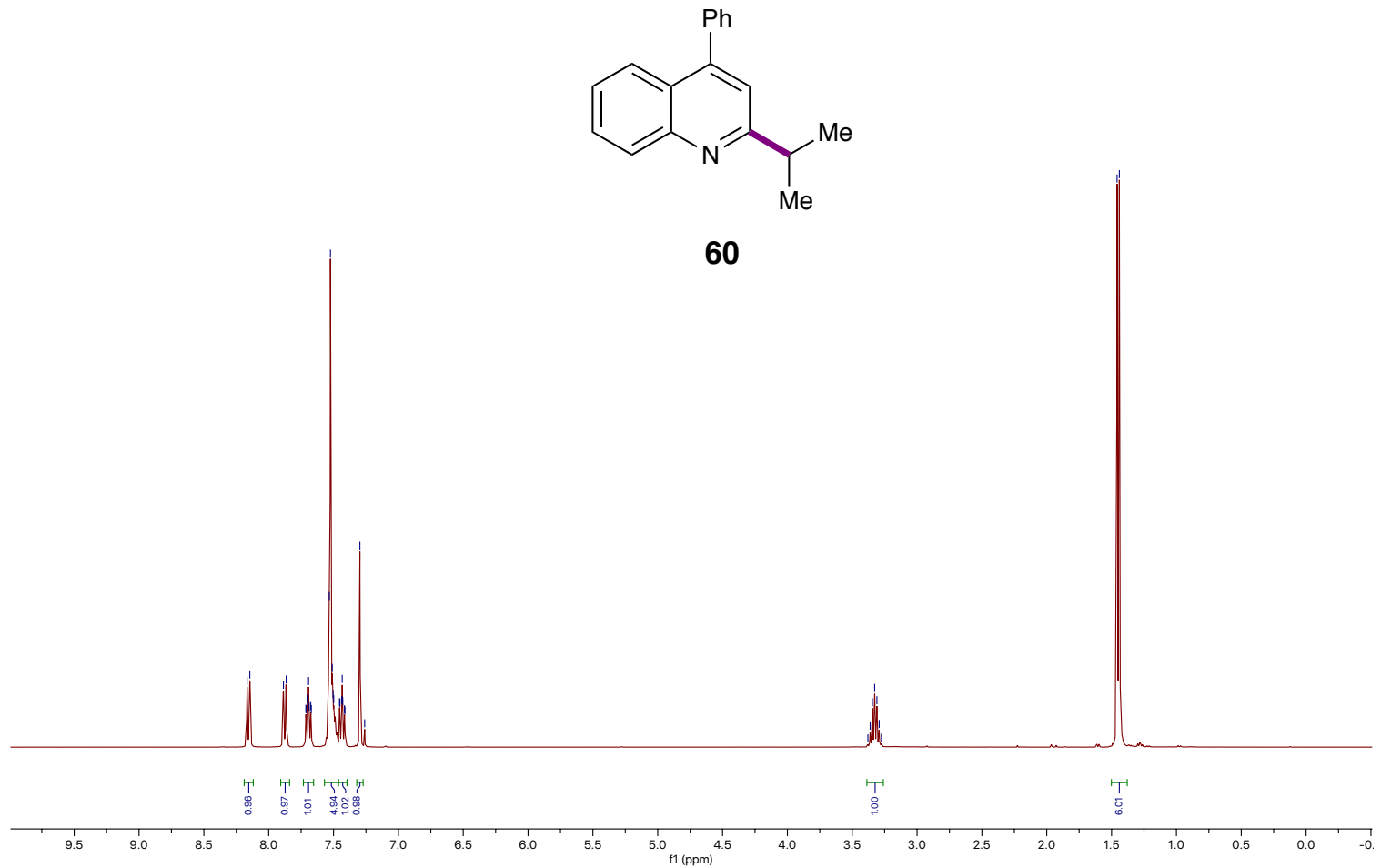

w-3-86CP-C
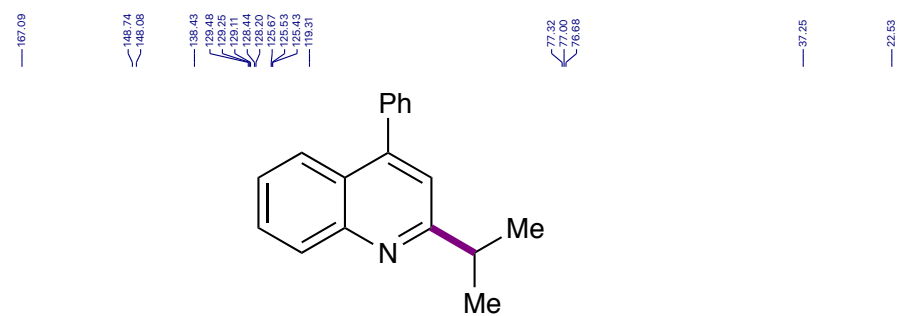

60
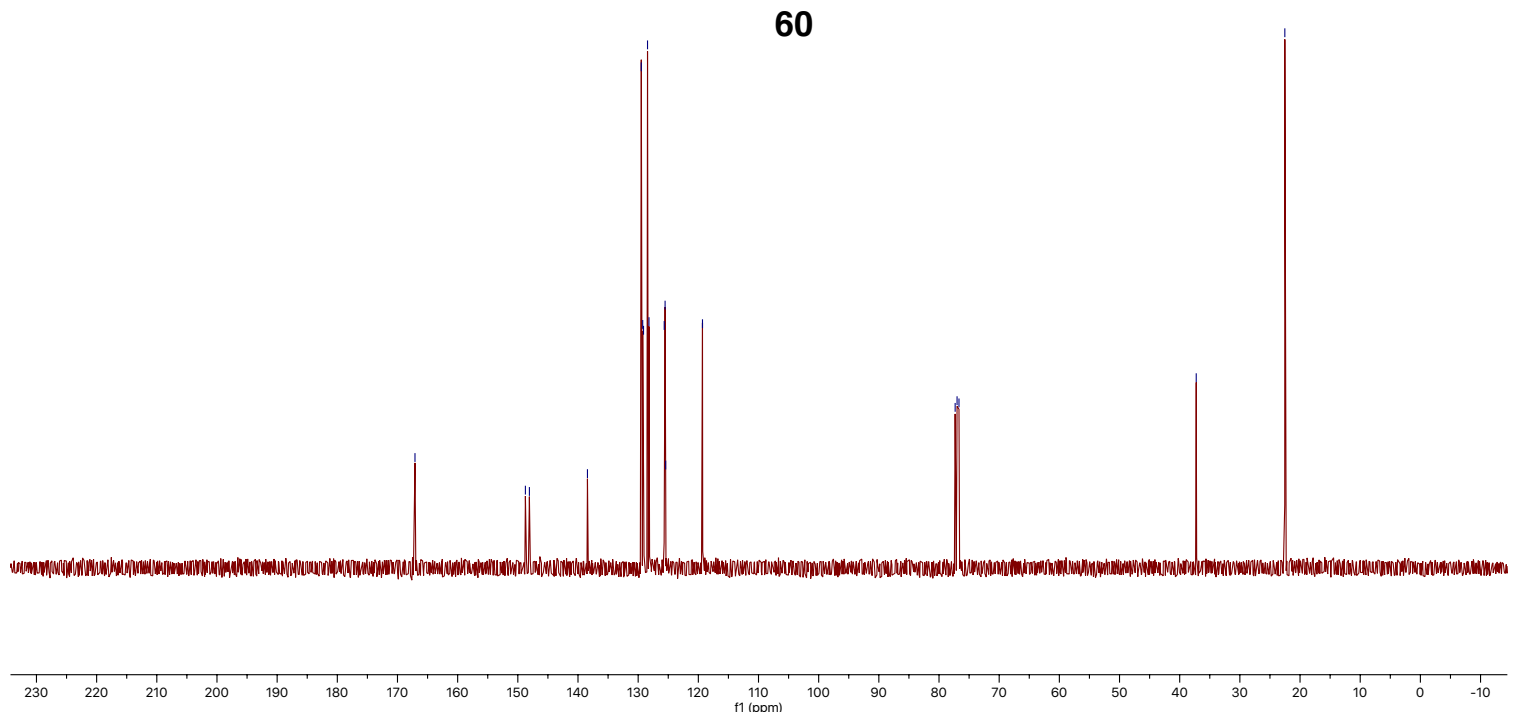
<smiles>COc1cc(C(C)C)nc2ccccc12</smiles>

\section{1}

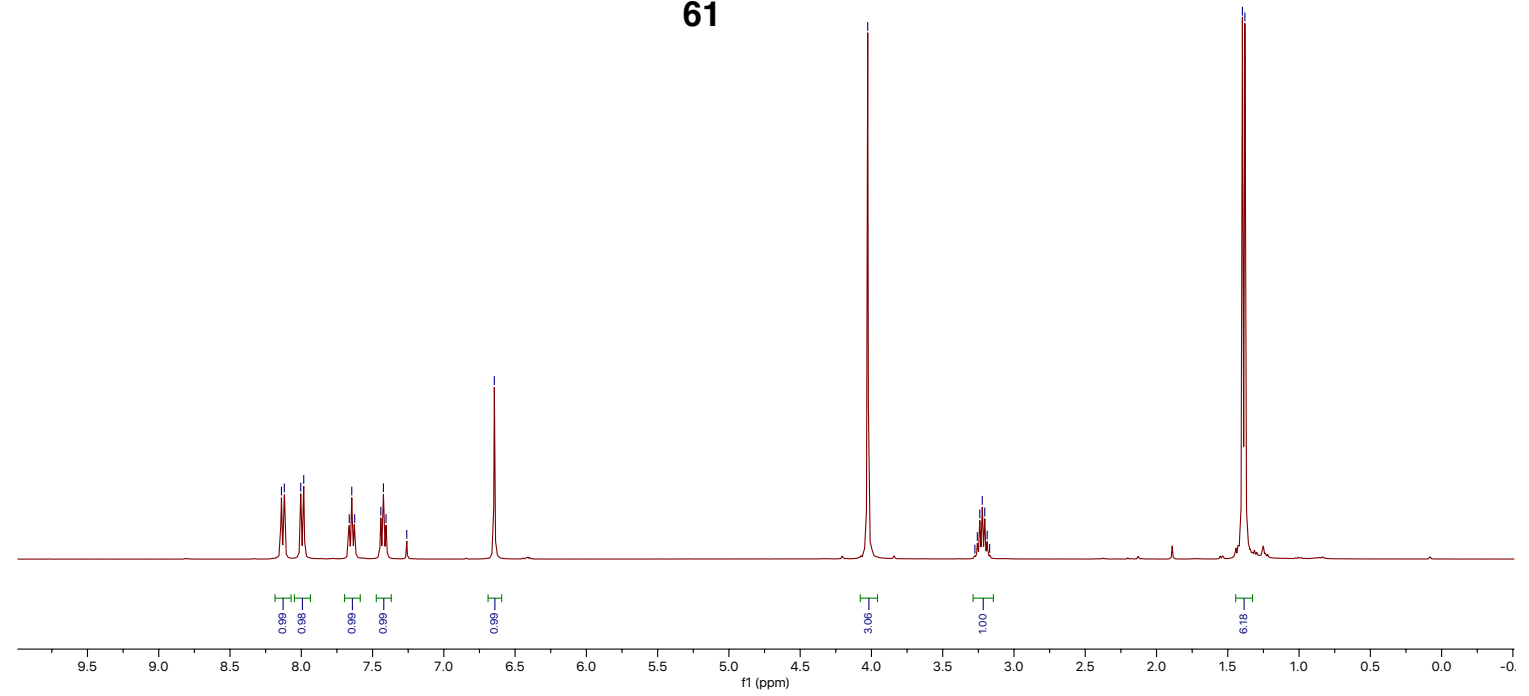

w-3-61p-c

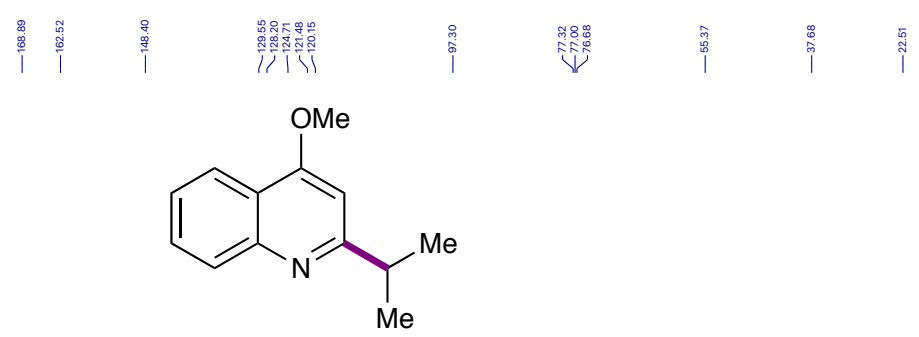

61

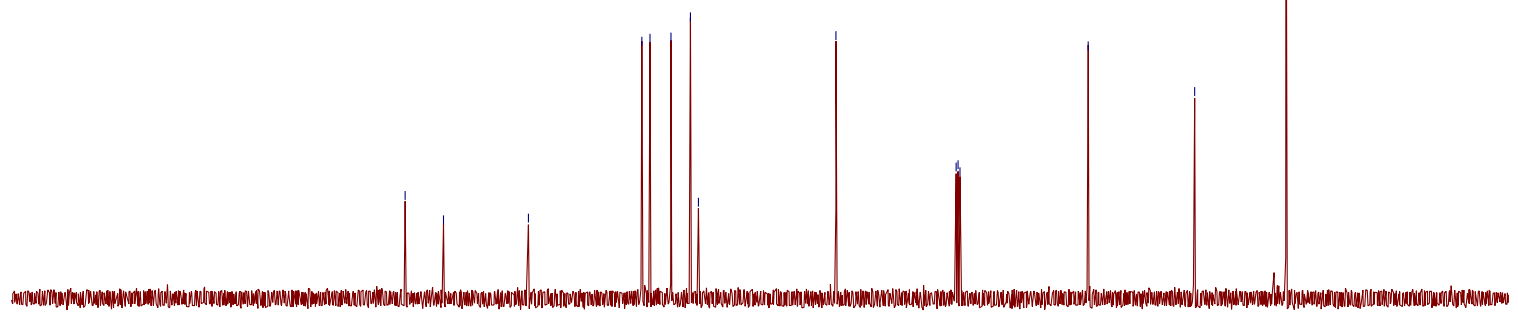




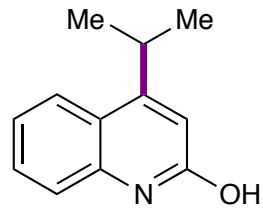

62

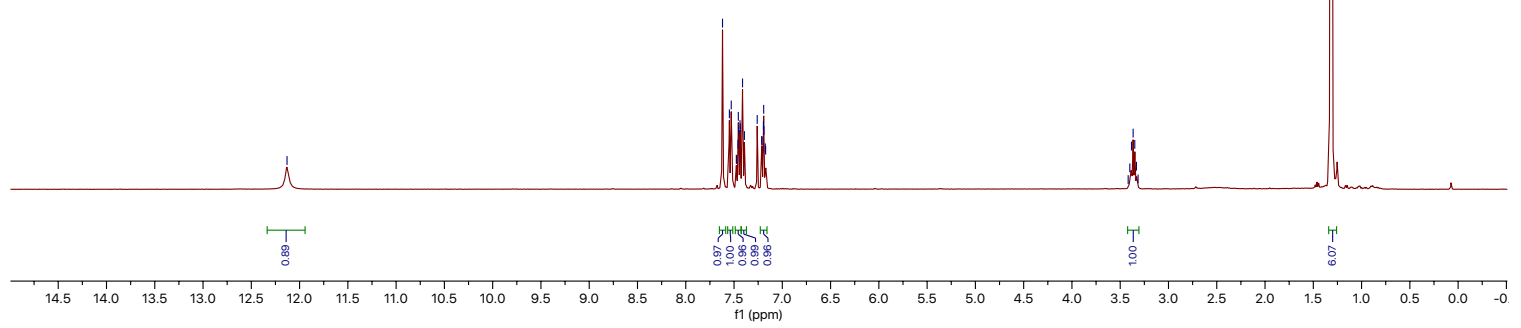

w-3-91cp-C
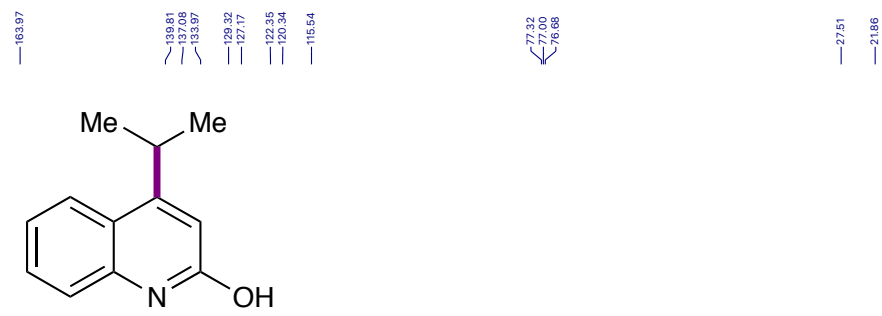

62

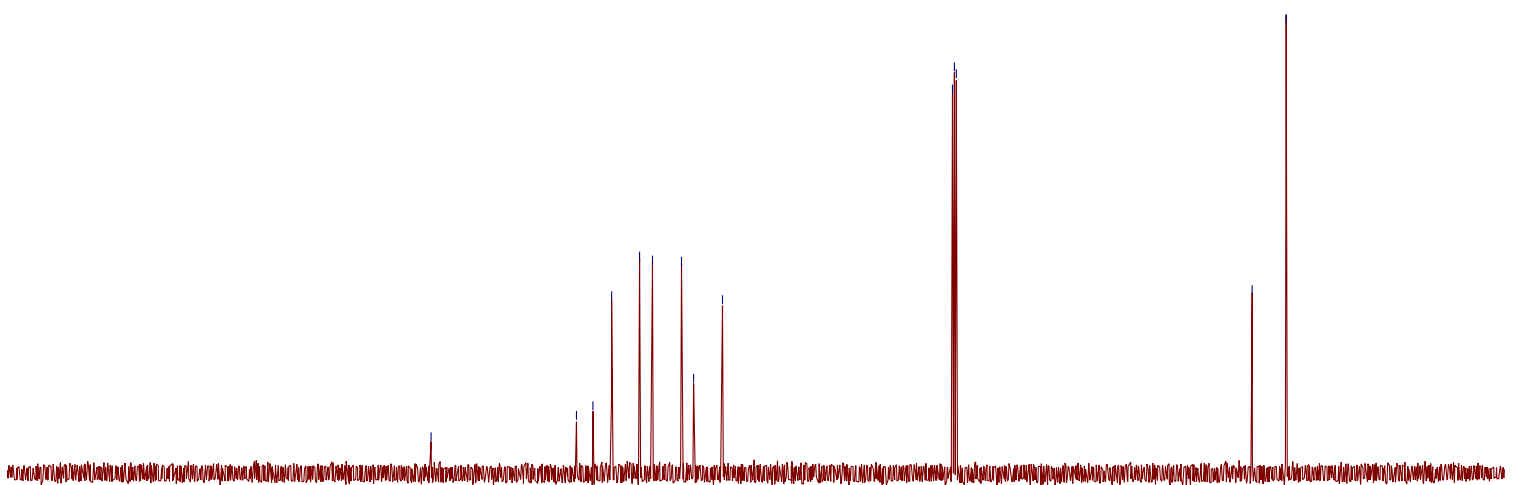

230 110
$\mathrm{f} 1(\mathrm{ppm})$ 
2011746-w-3-57p.10.fid

ن

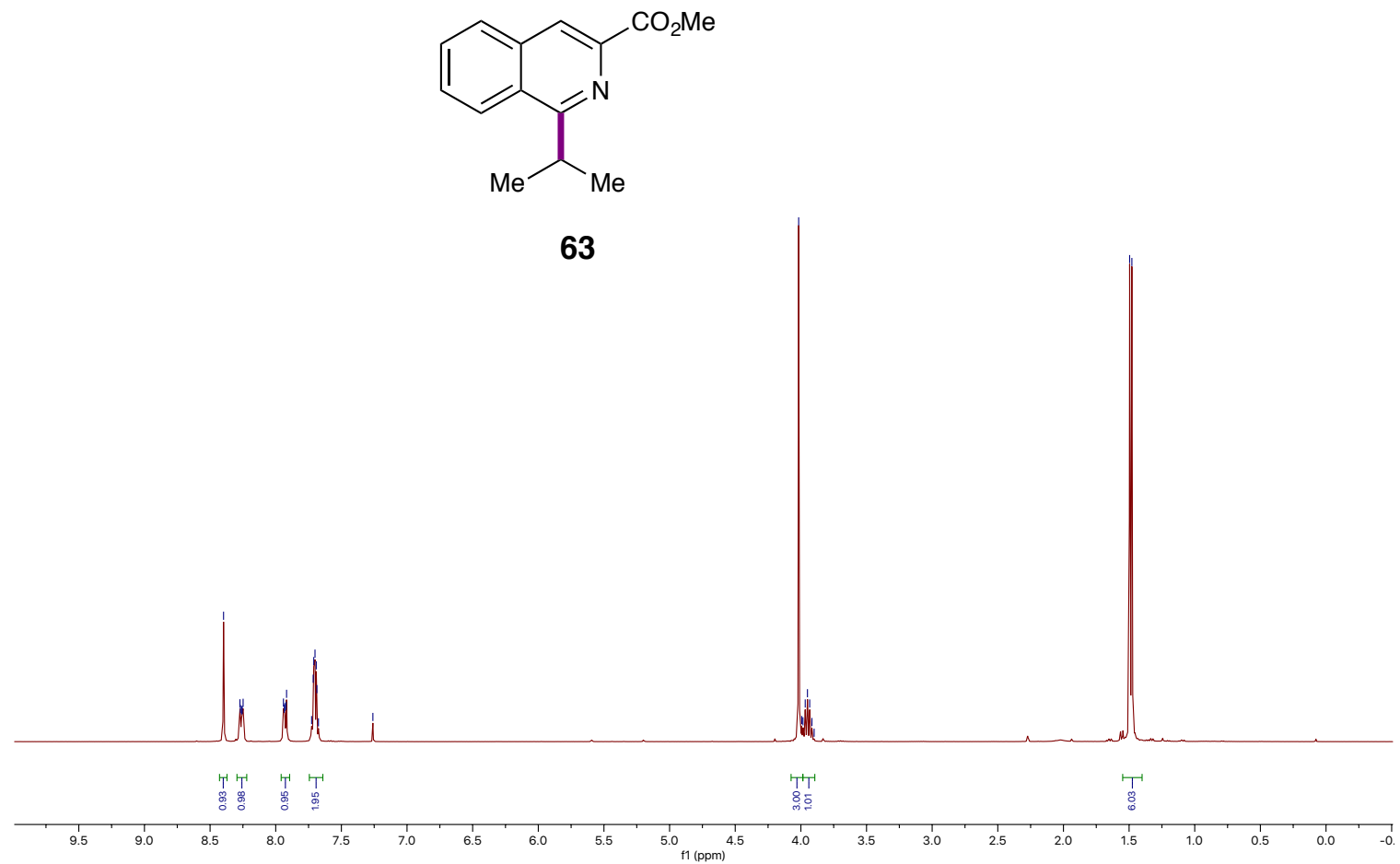

$-w-3-57-p-c$

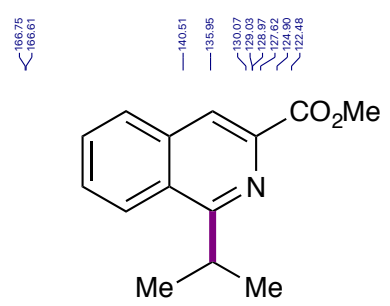

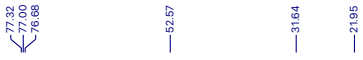

63

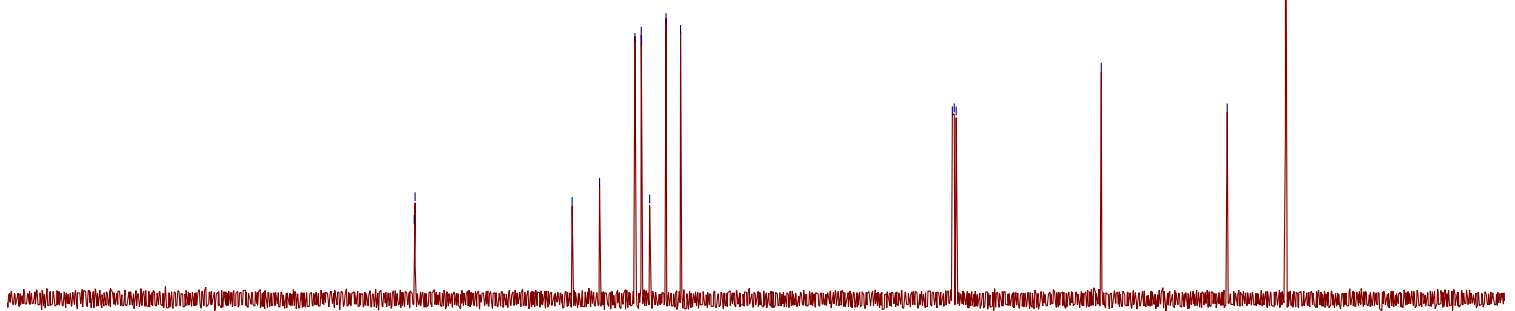

230

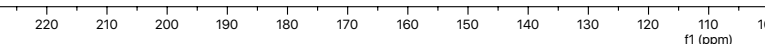



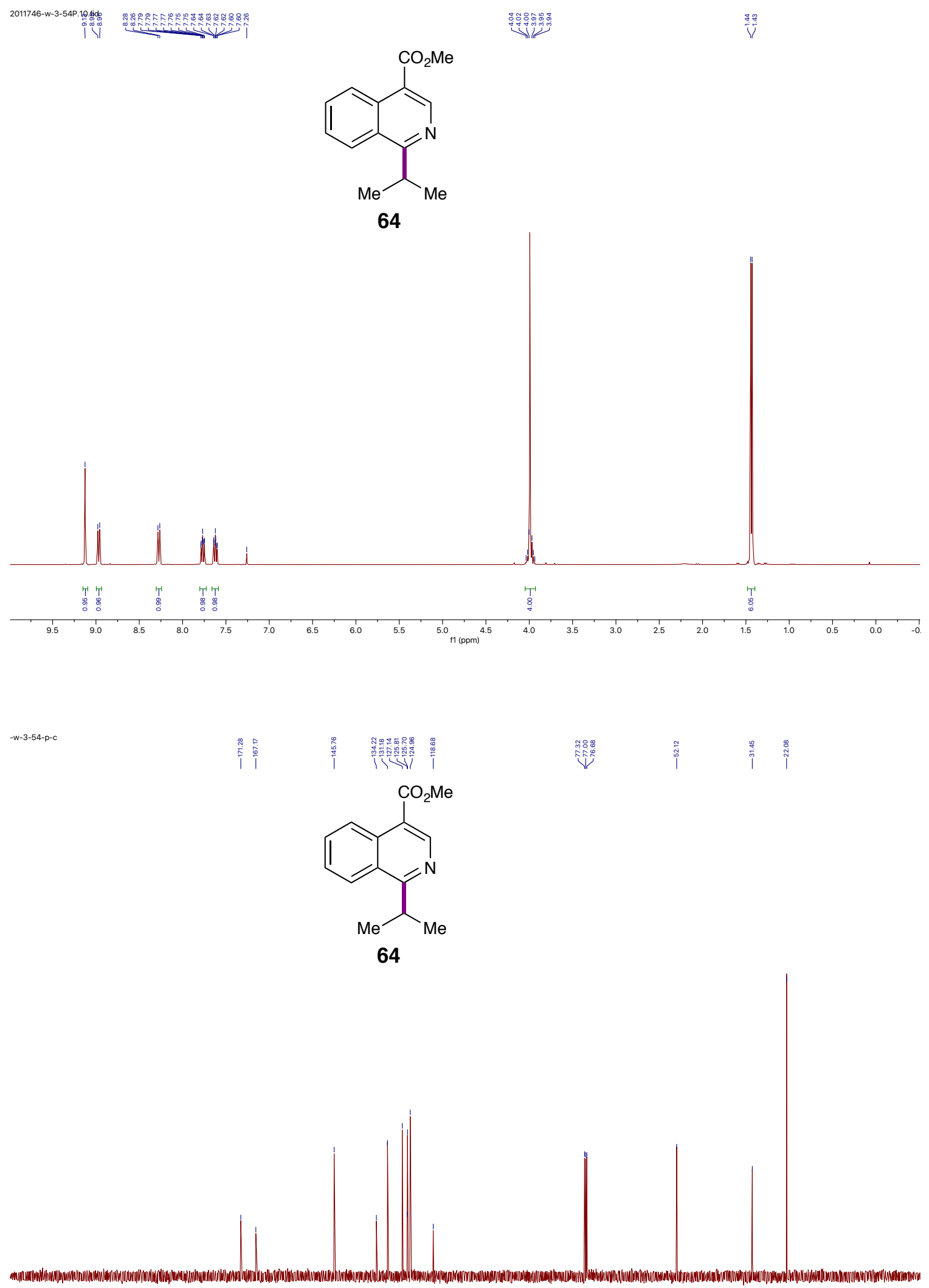

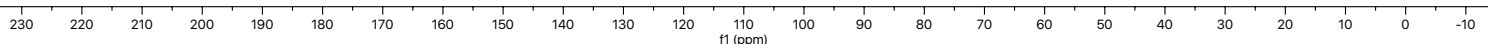



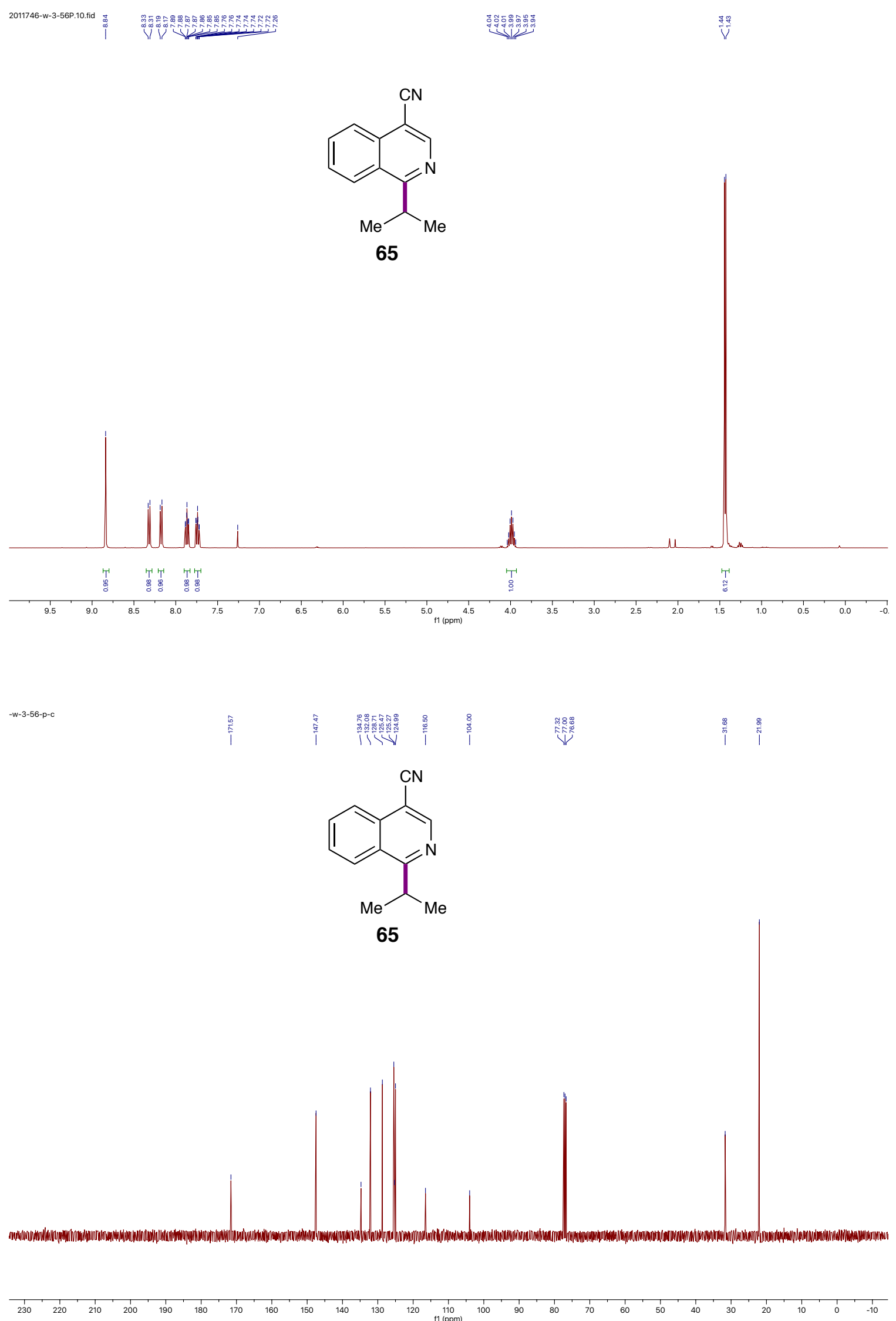

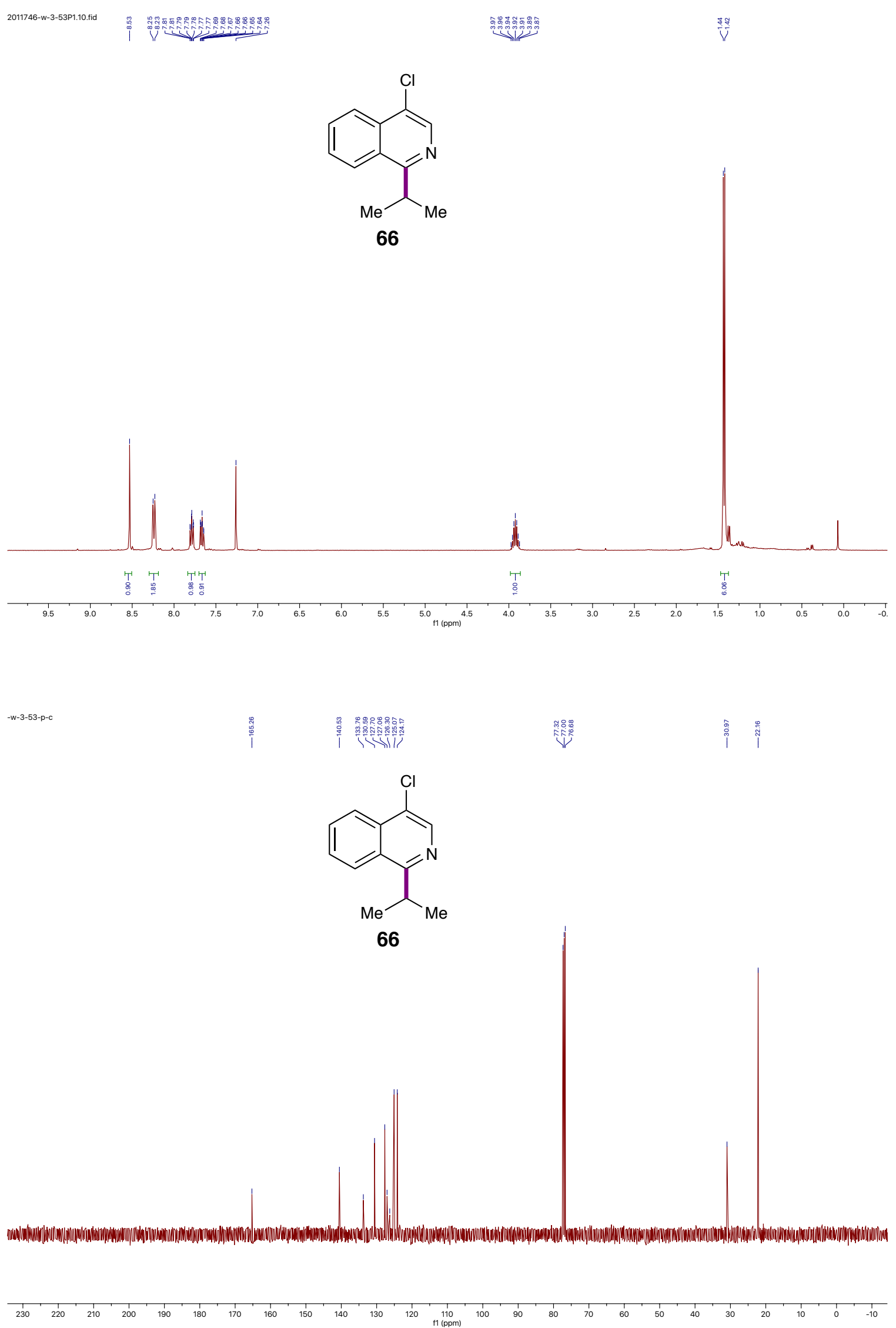
2011746-W-3-50B-P-re.10.fid

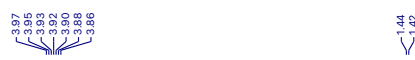<smiles>CC(C)c1ncc(Br)c2ccccc12</smiles>

67

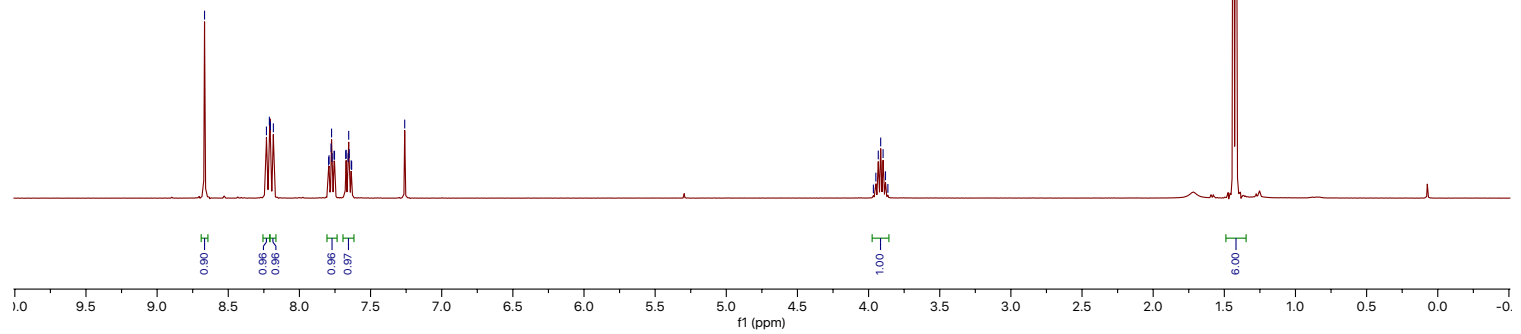

$-\mathrm{w}-3-50-\mathrm{p}-\mathrm{c}$

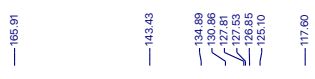

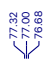

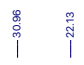<smiles>CC(C)c1ncc(Br)c2ccccc12</smiles>

67

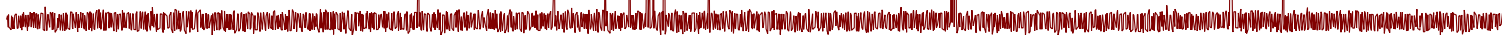

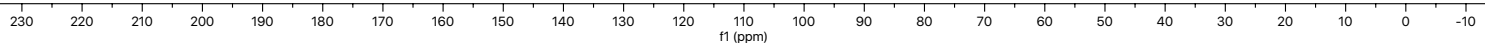


2011746-w-3-52-P.10.fid

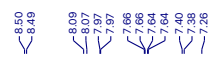

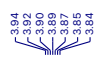

䰹

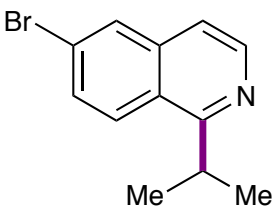

68

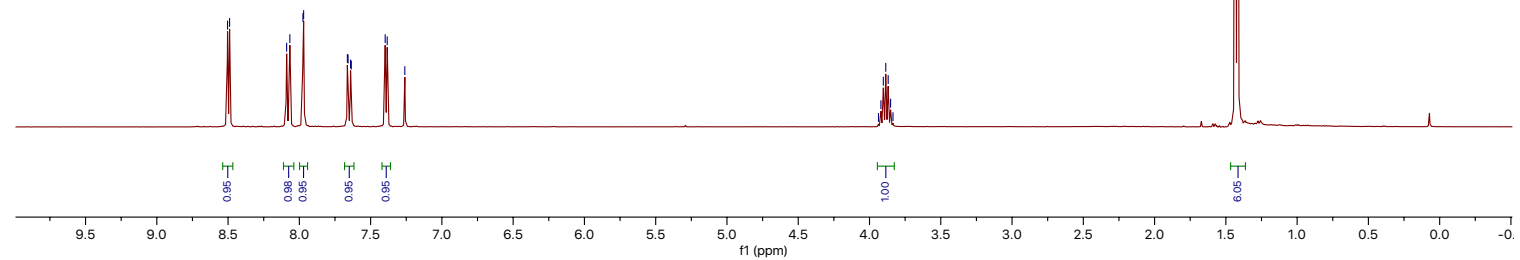

-w-3-52-p-c

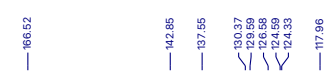
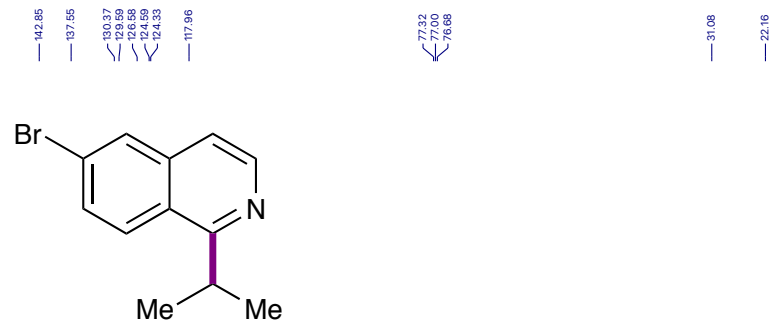

68

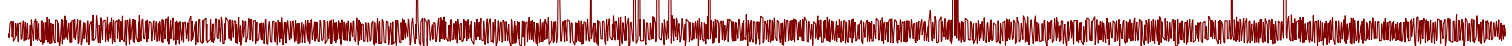

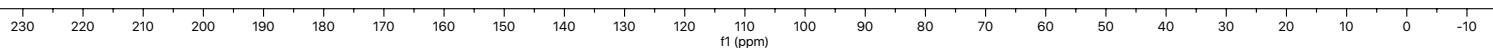


2011746-w-4-3EP.10.fid

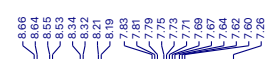

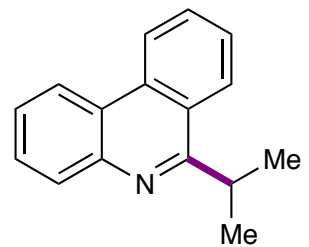

69

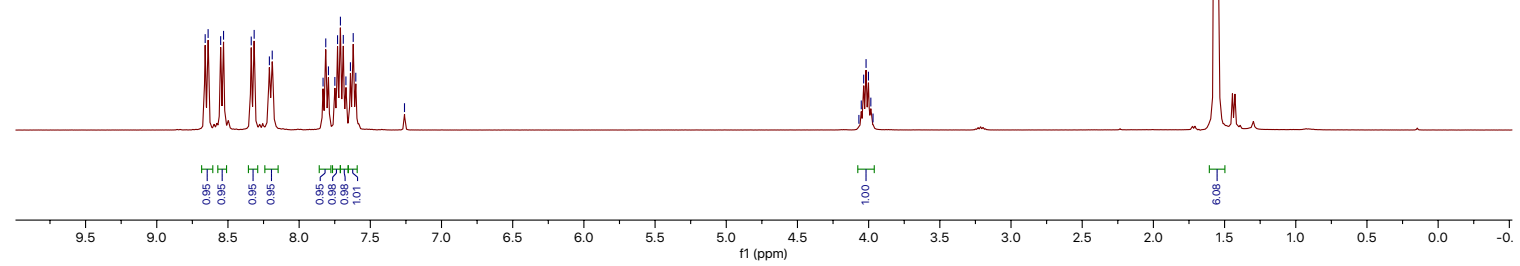

W-4-3EP-C
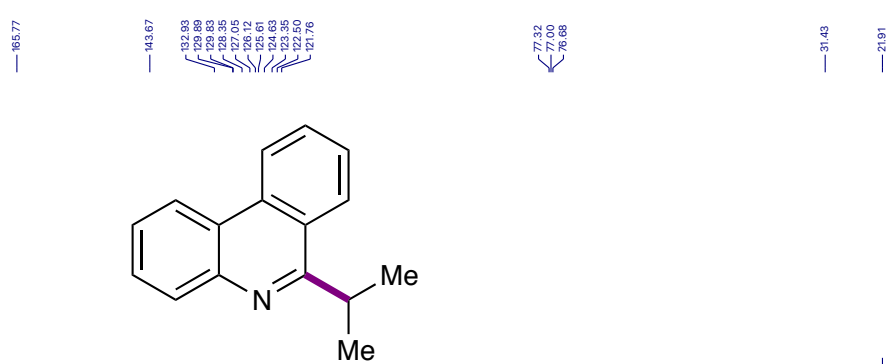

69

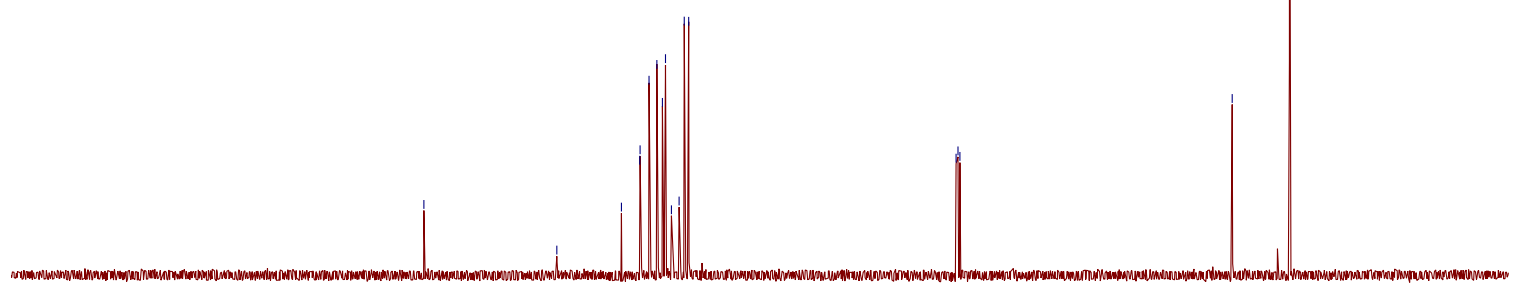

230

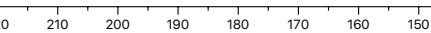

$150 \quad 140$

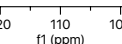




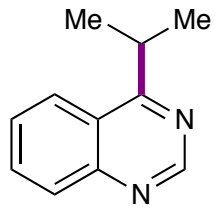

70

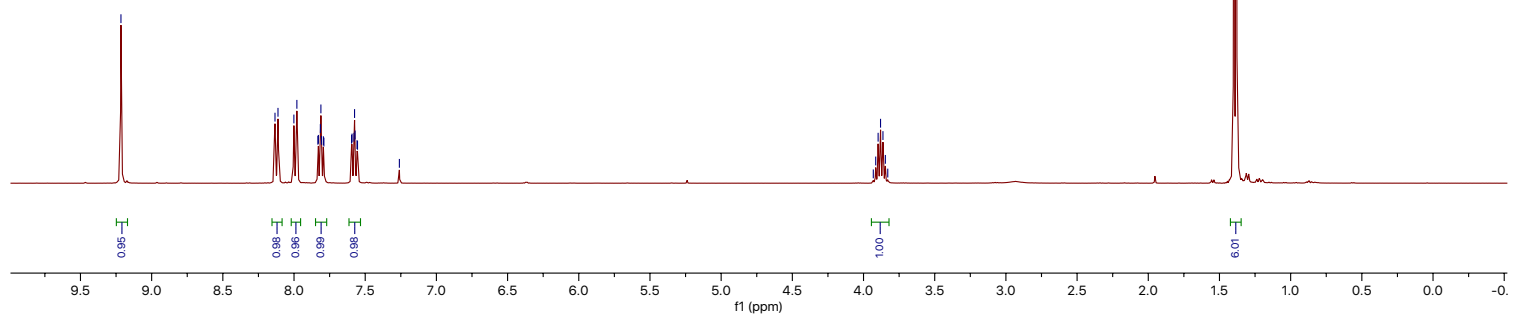

w-3-68p1-C

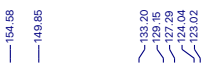

党
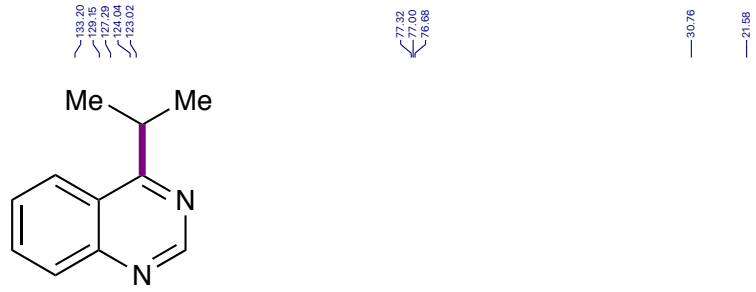

70

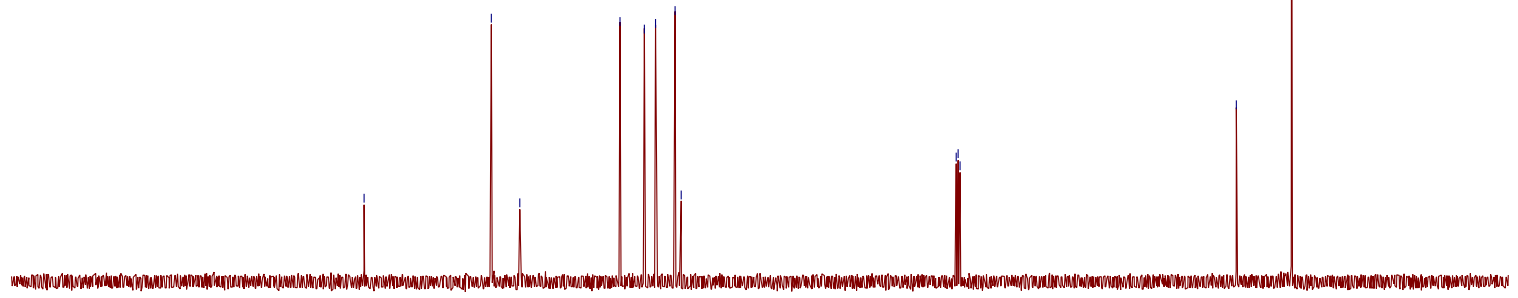

230

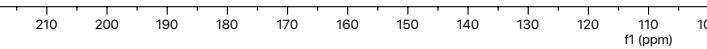


<smiles>CC(C)c1nc(Cl)nc2ccccc12</smiles>

71

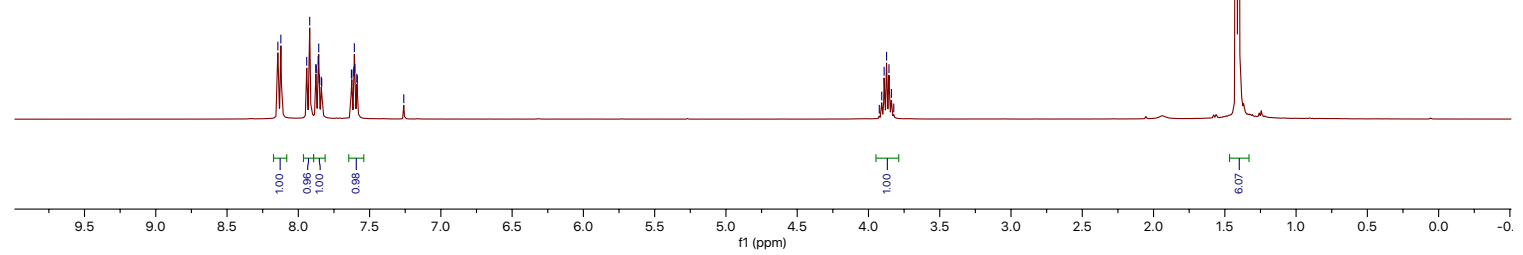

w-3-70p-C

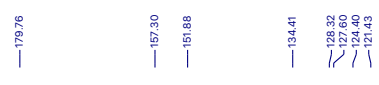

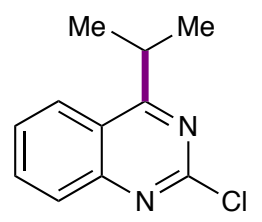

71

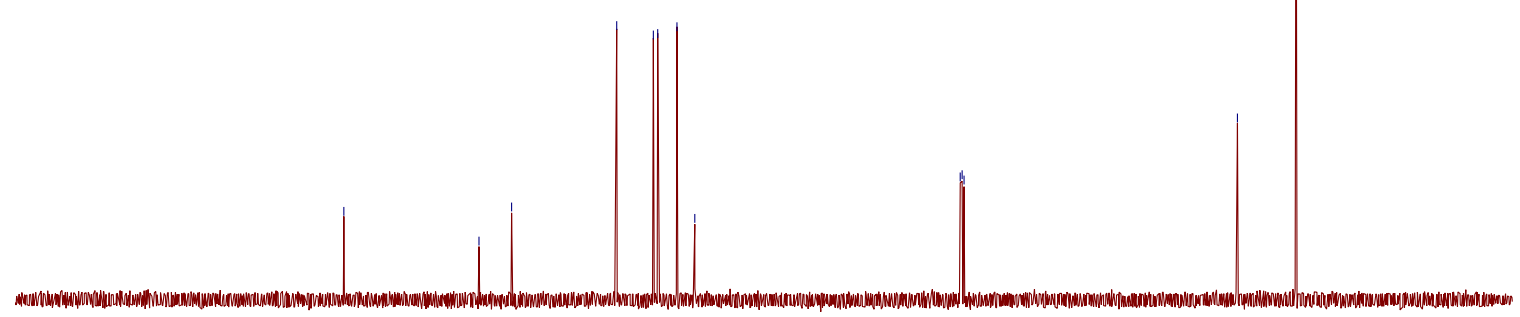

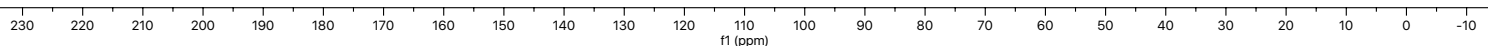



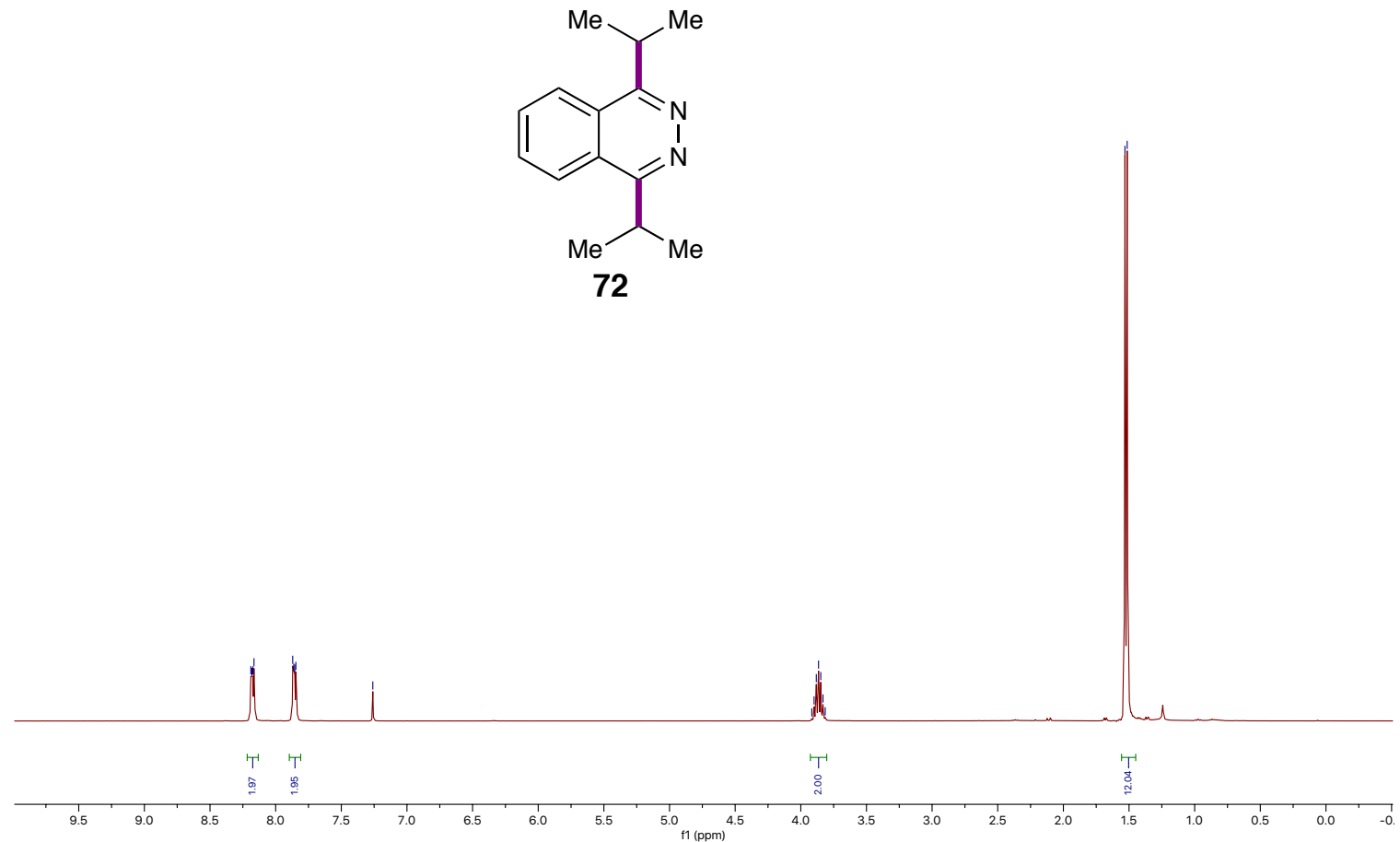

-w-3-93ap-c<smiles>CC(C)c1nnc(C(C)C)c2ccccc12</smiles>

72

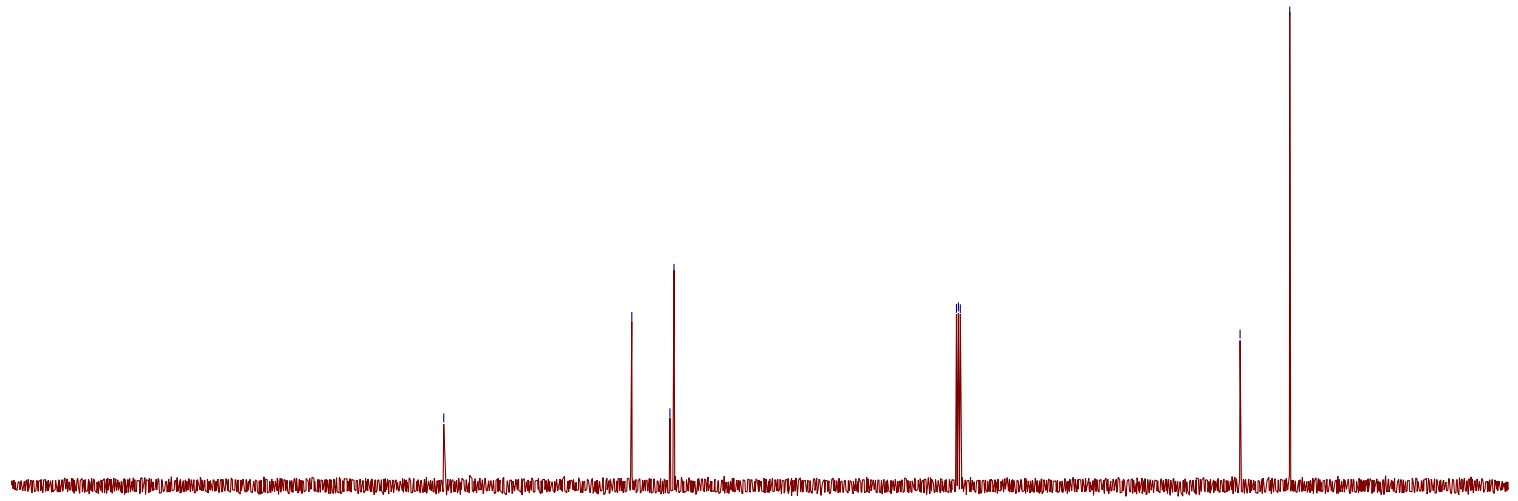

230

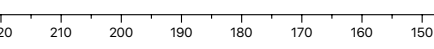




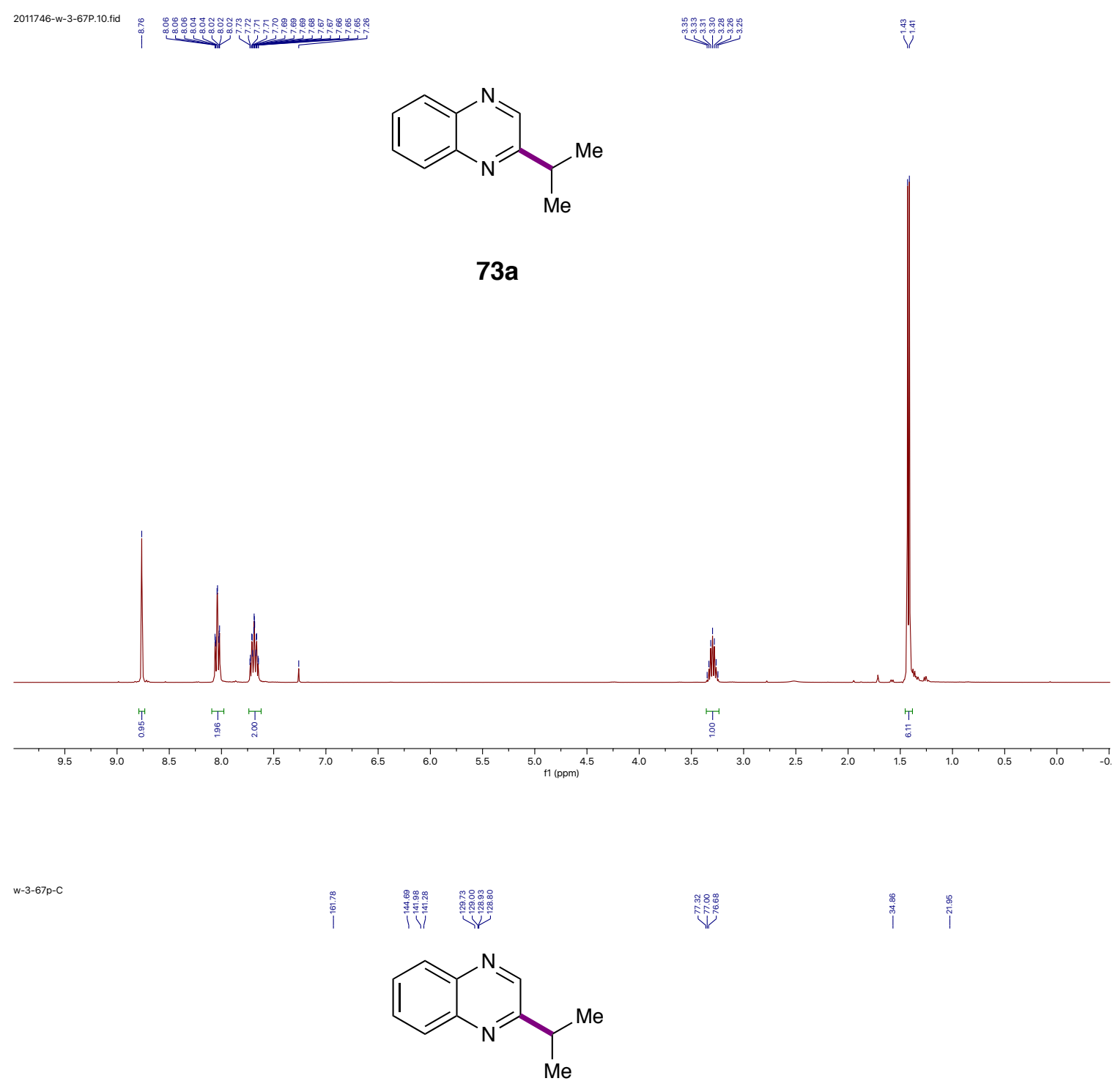

73a

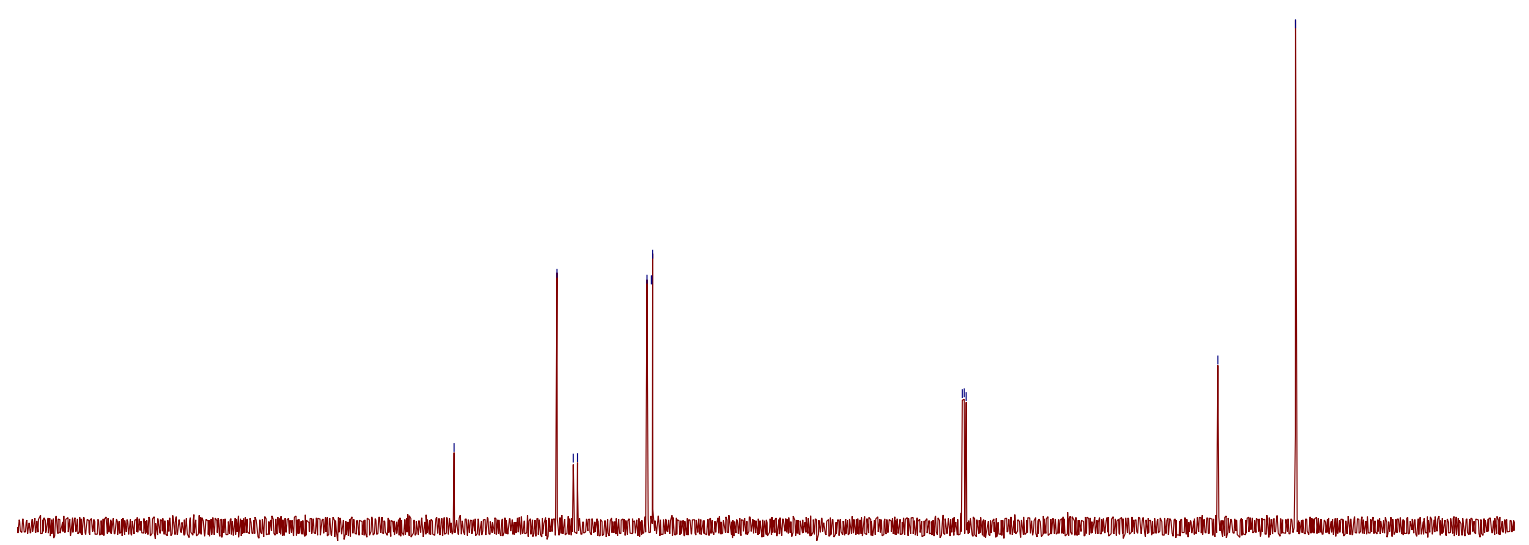

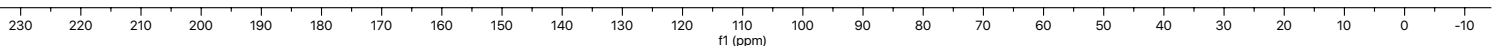


2011746-w-4-05HP1.10.fid

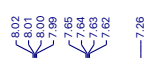

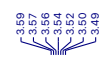

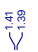

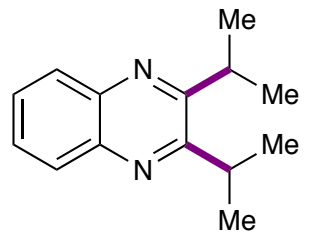

73b

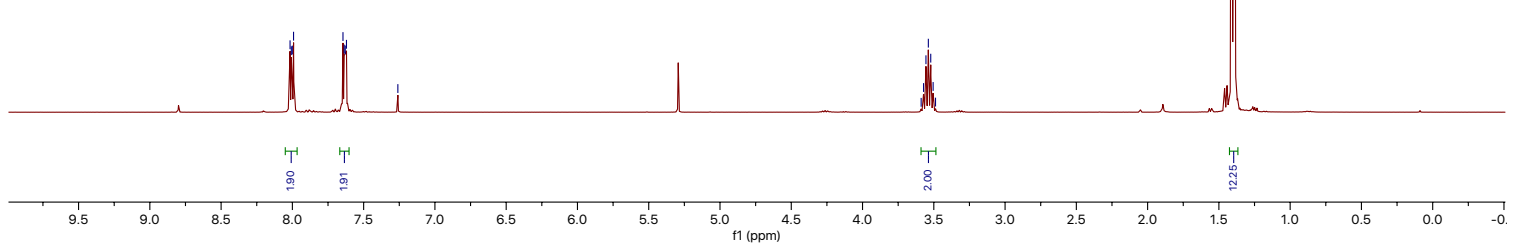

w-4-5-hp1

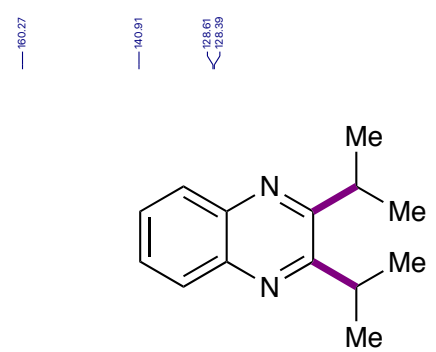

$73 b$

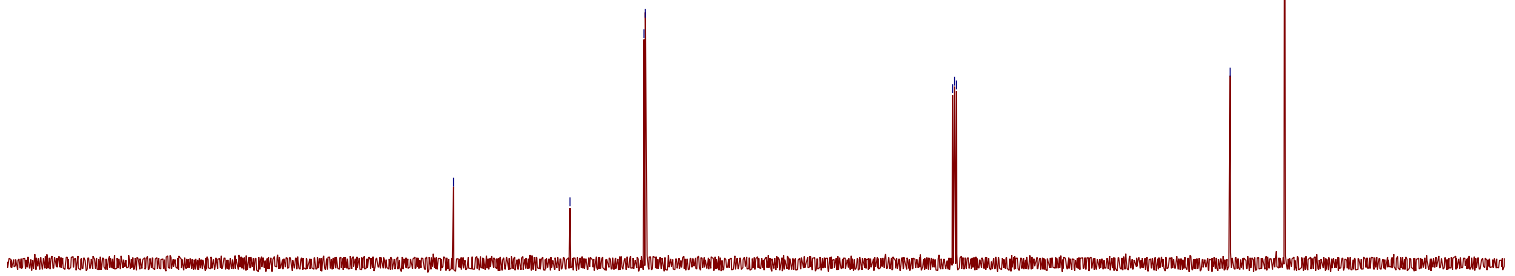

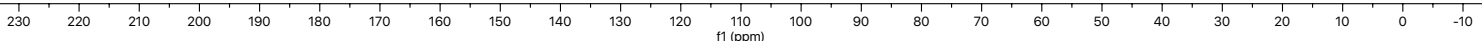




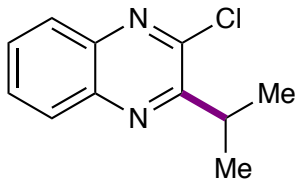

74

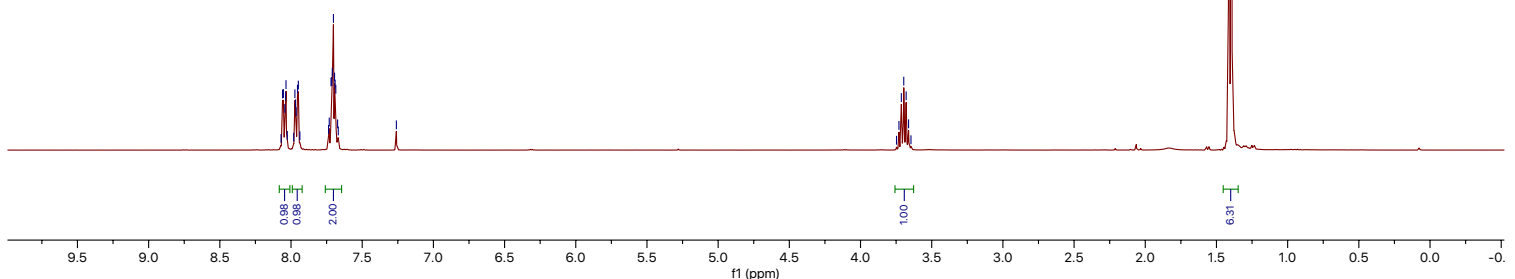

w-3-71p-C
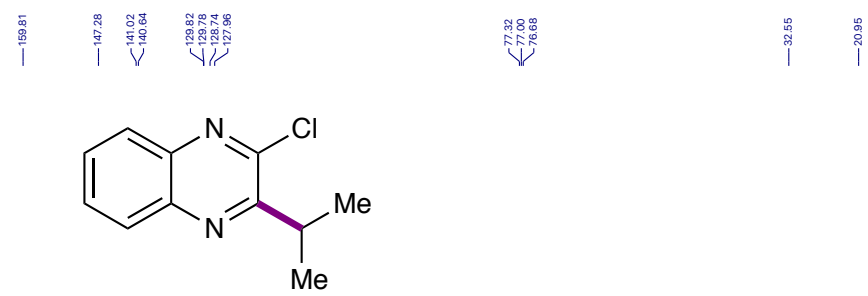

74

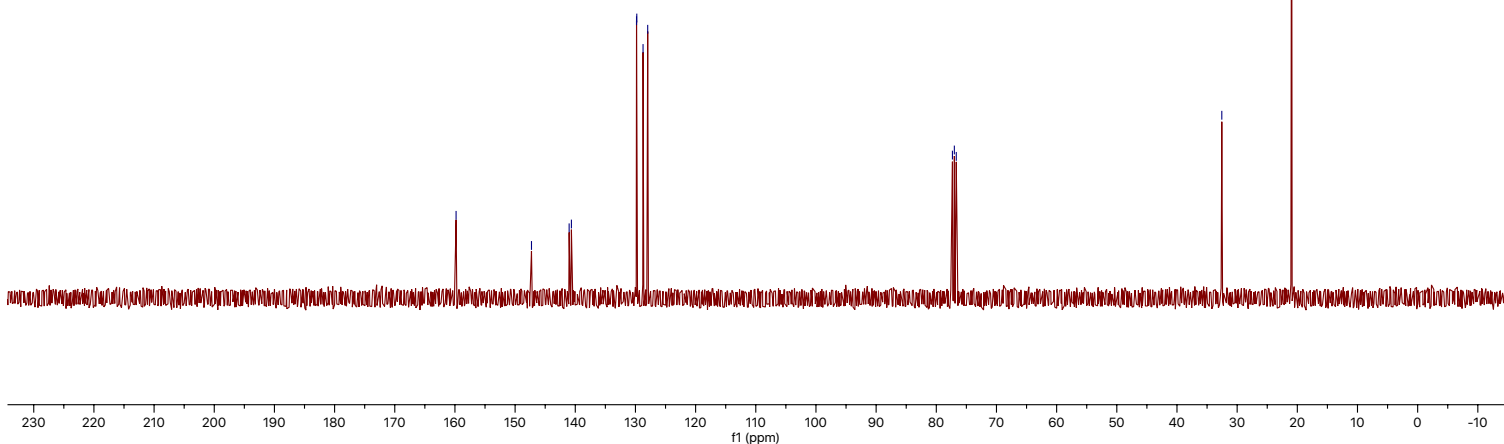




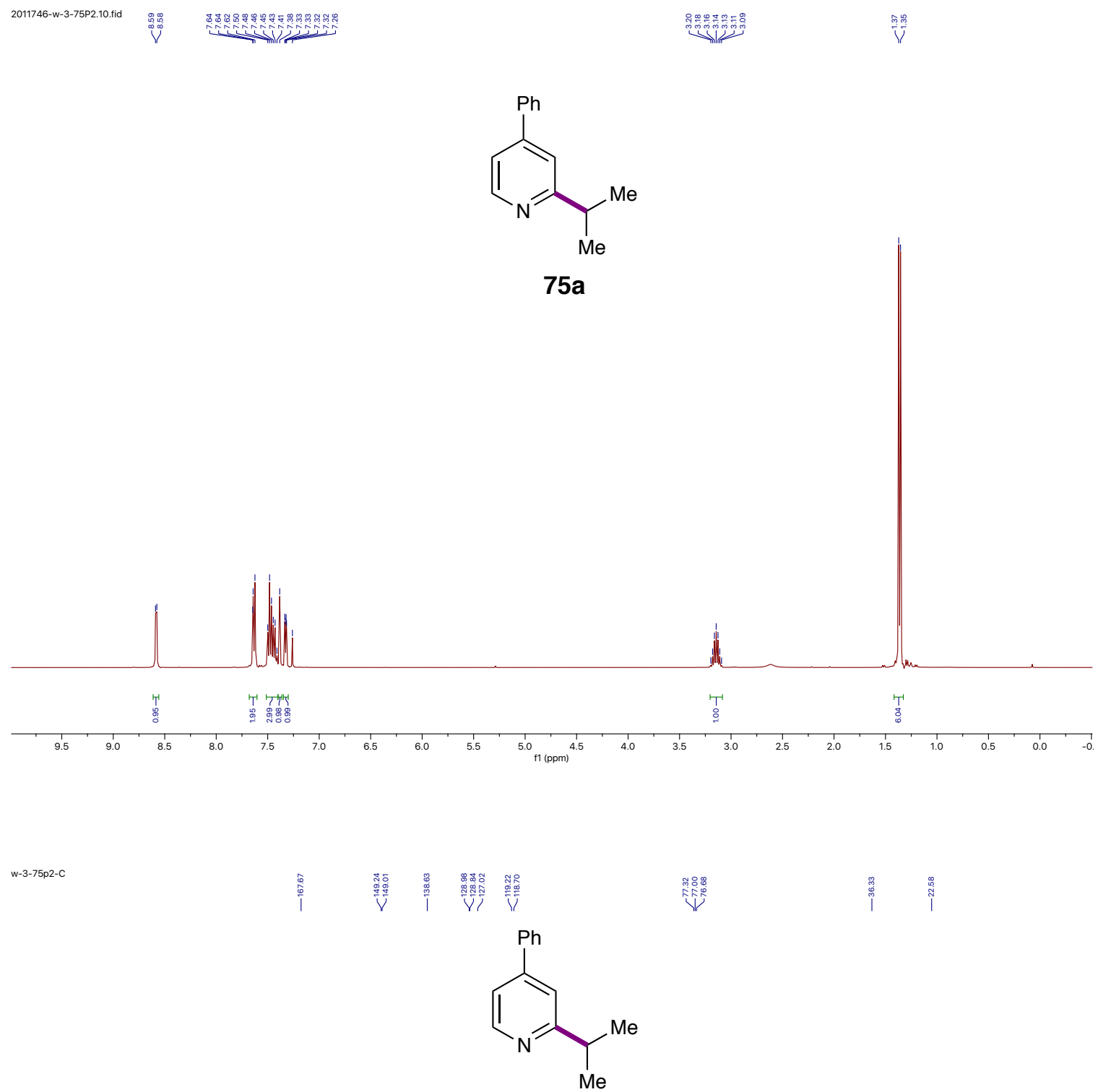

$75 a$
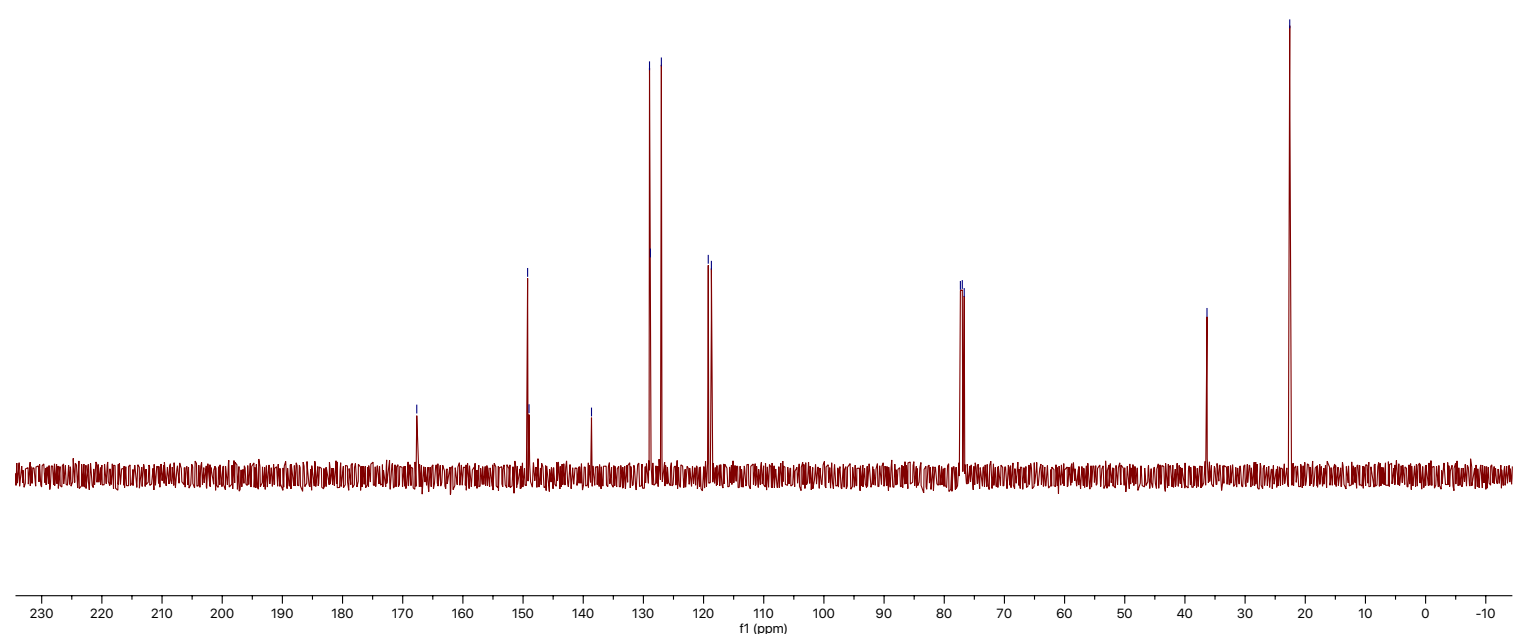


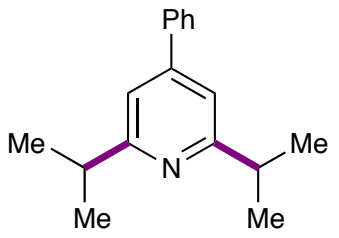

$75 b$

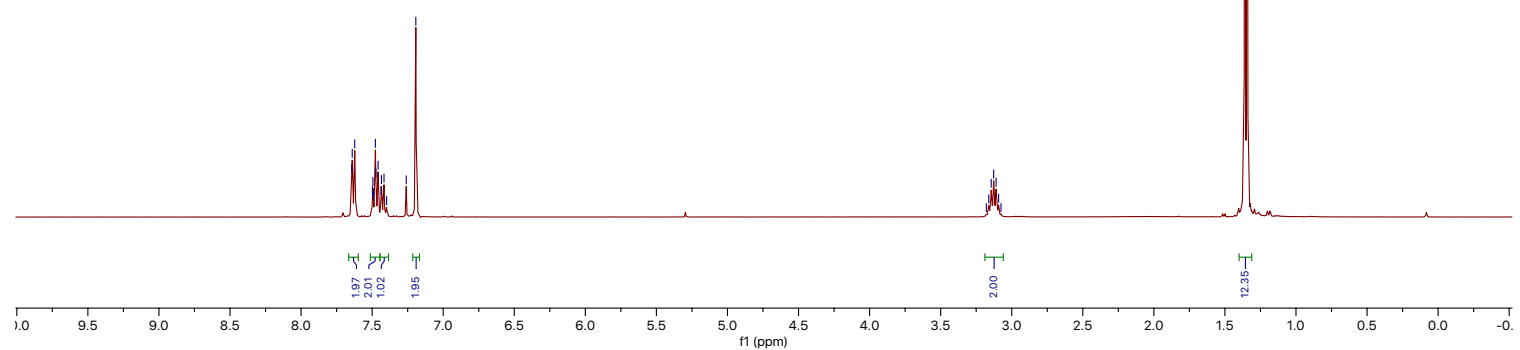

w-3-75p1-C
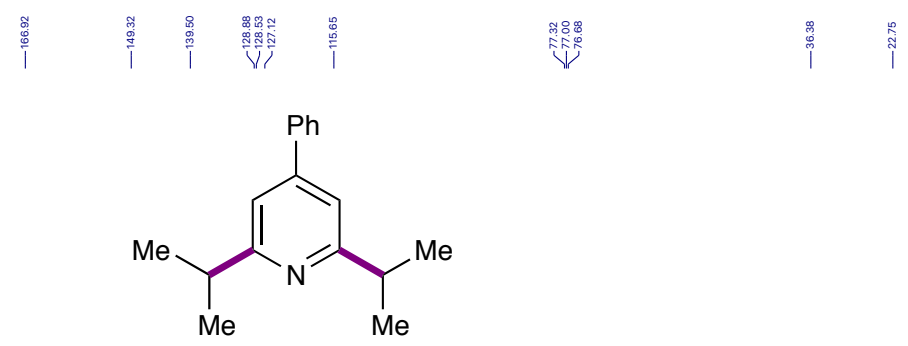

$75 b$

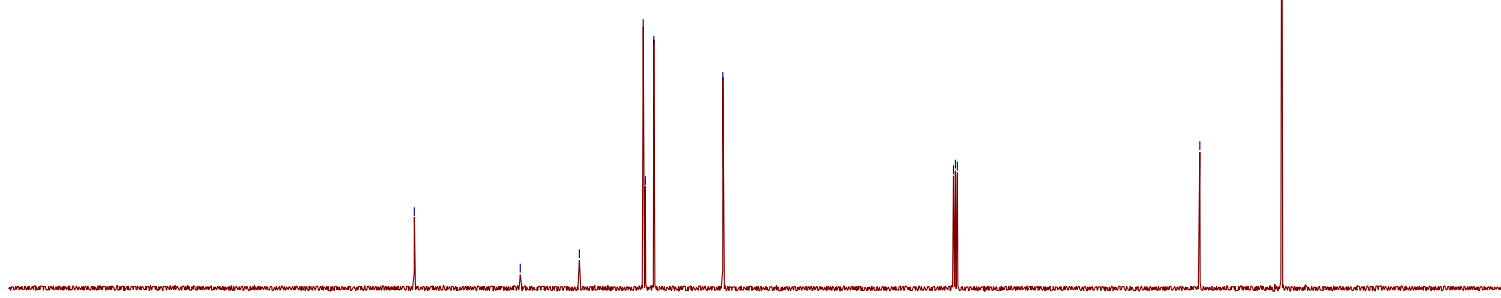




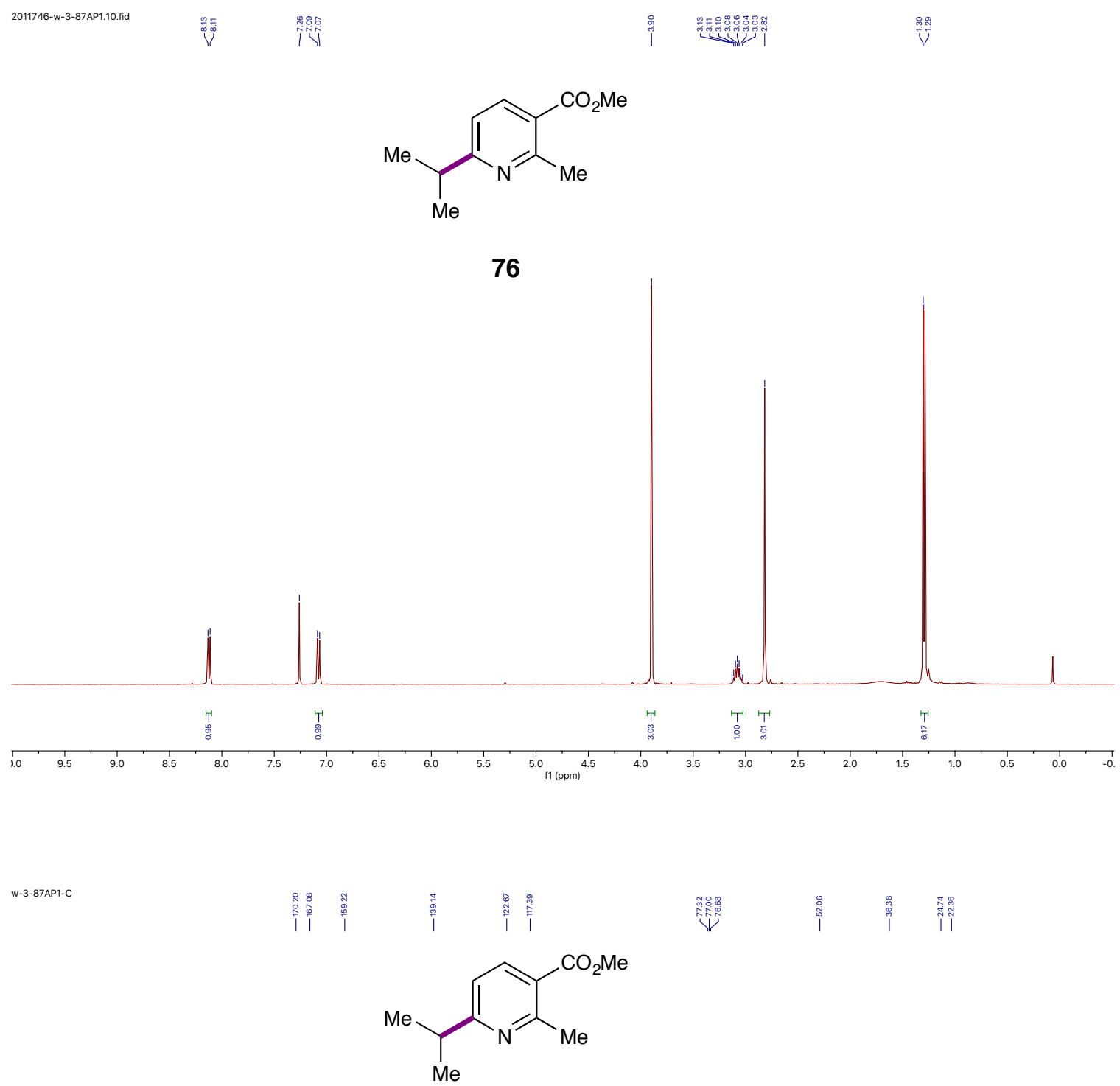

76

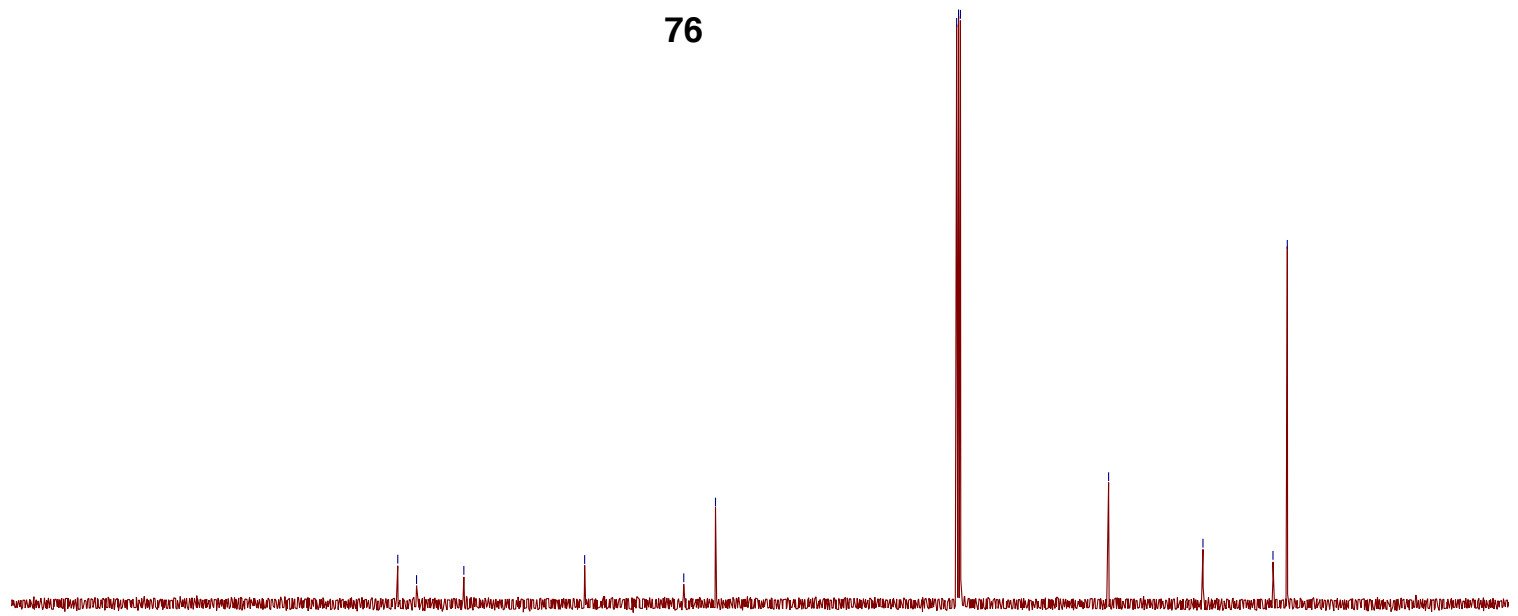

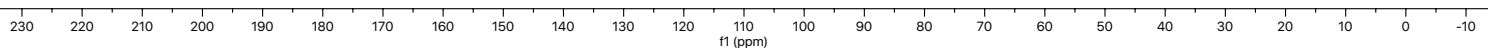



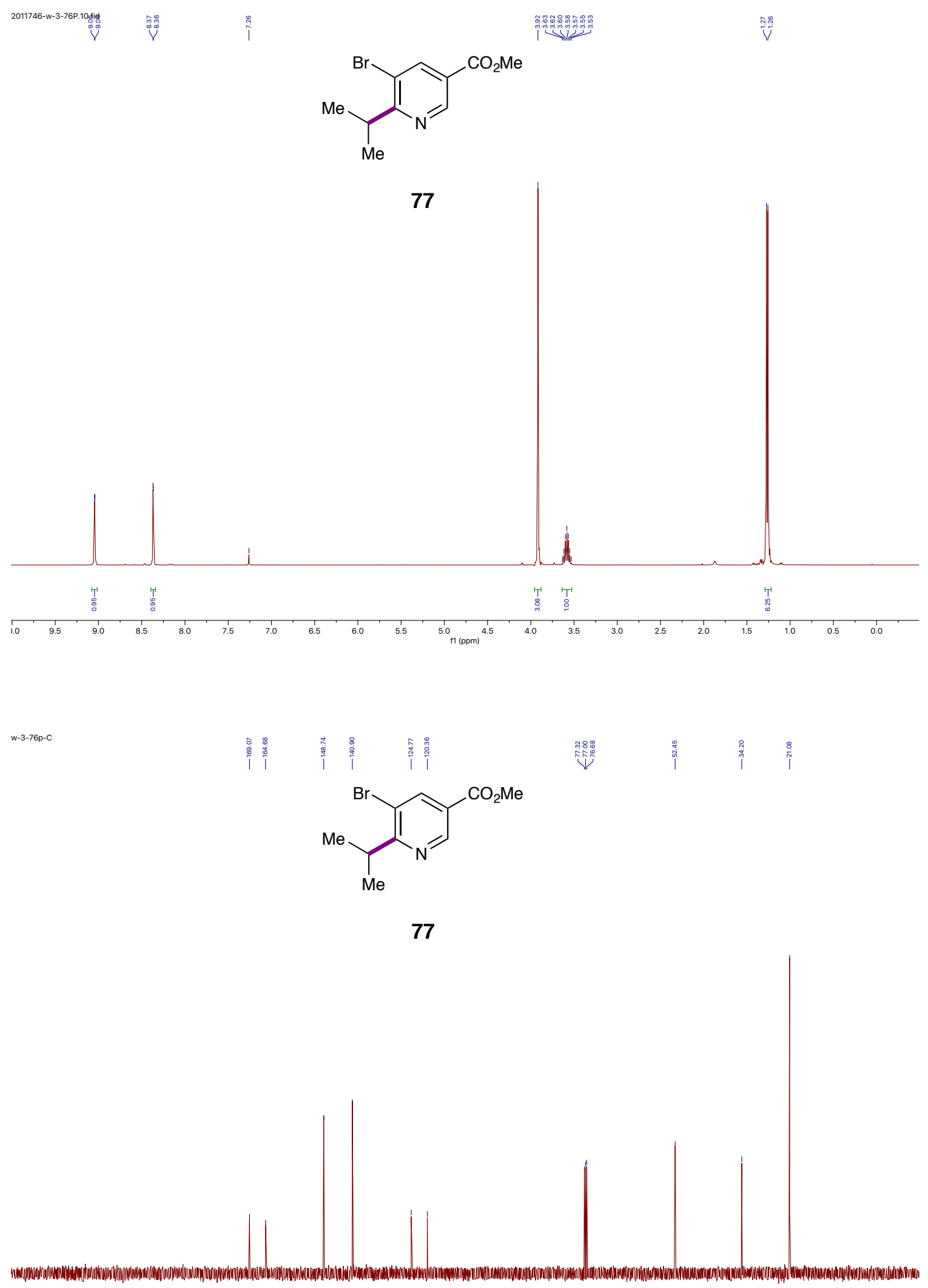

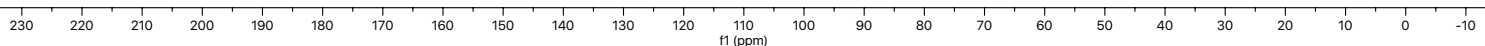




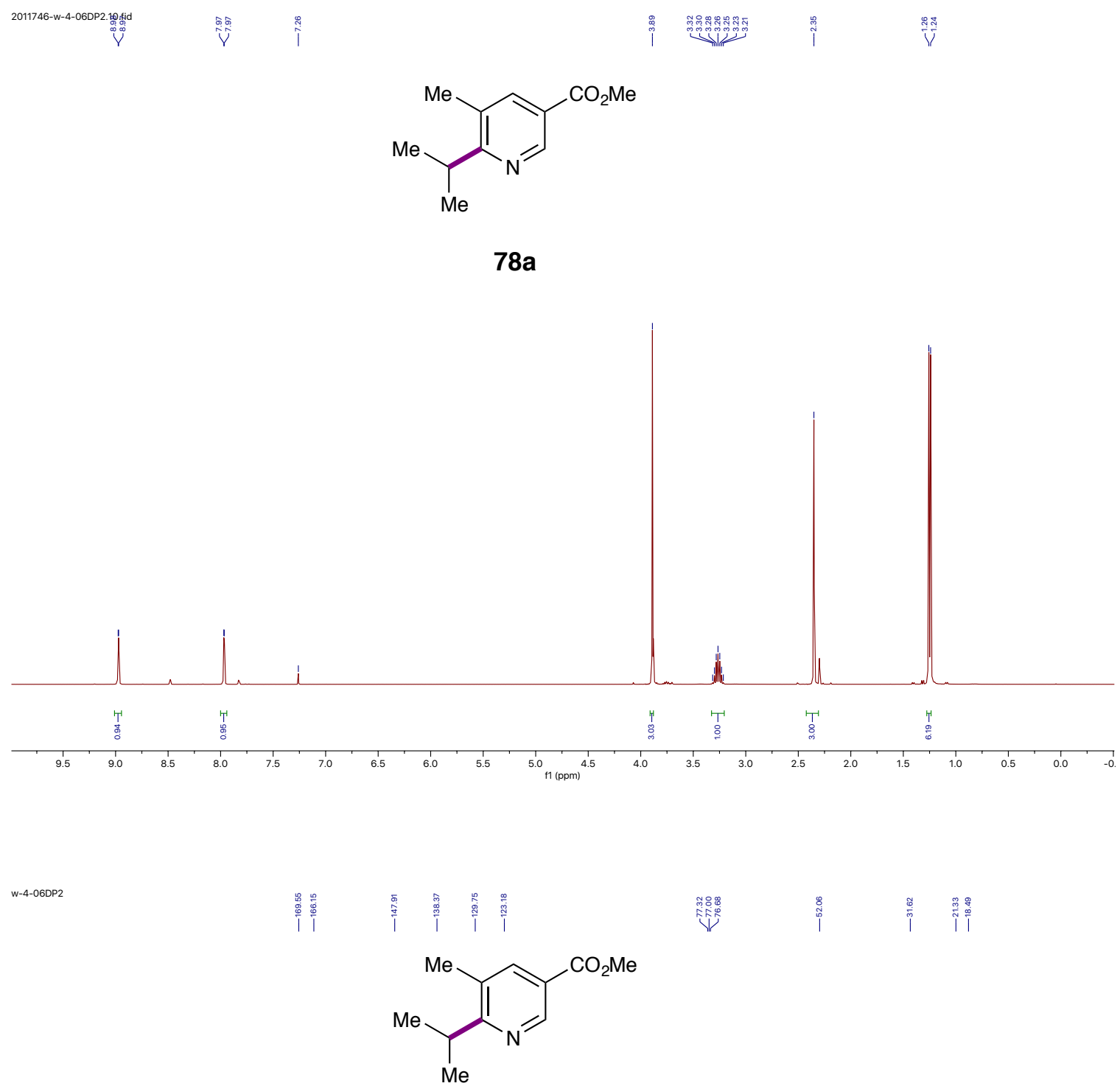

$78 a$

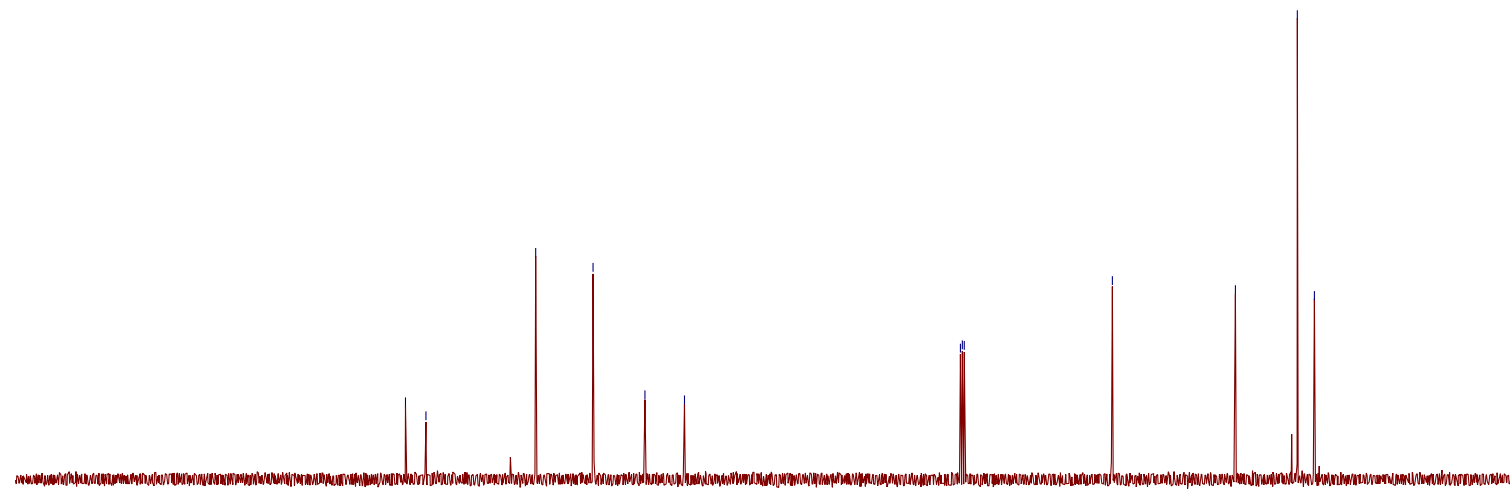

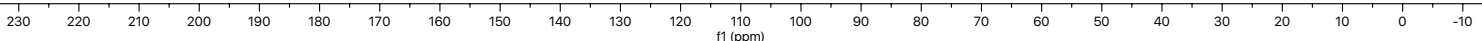



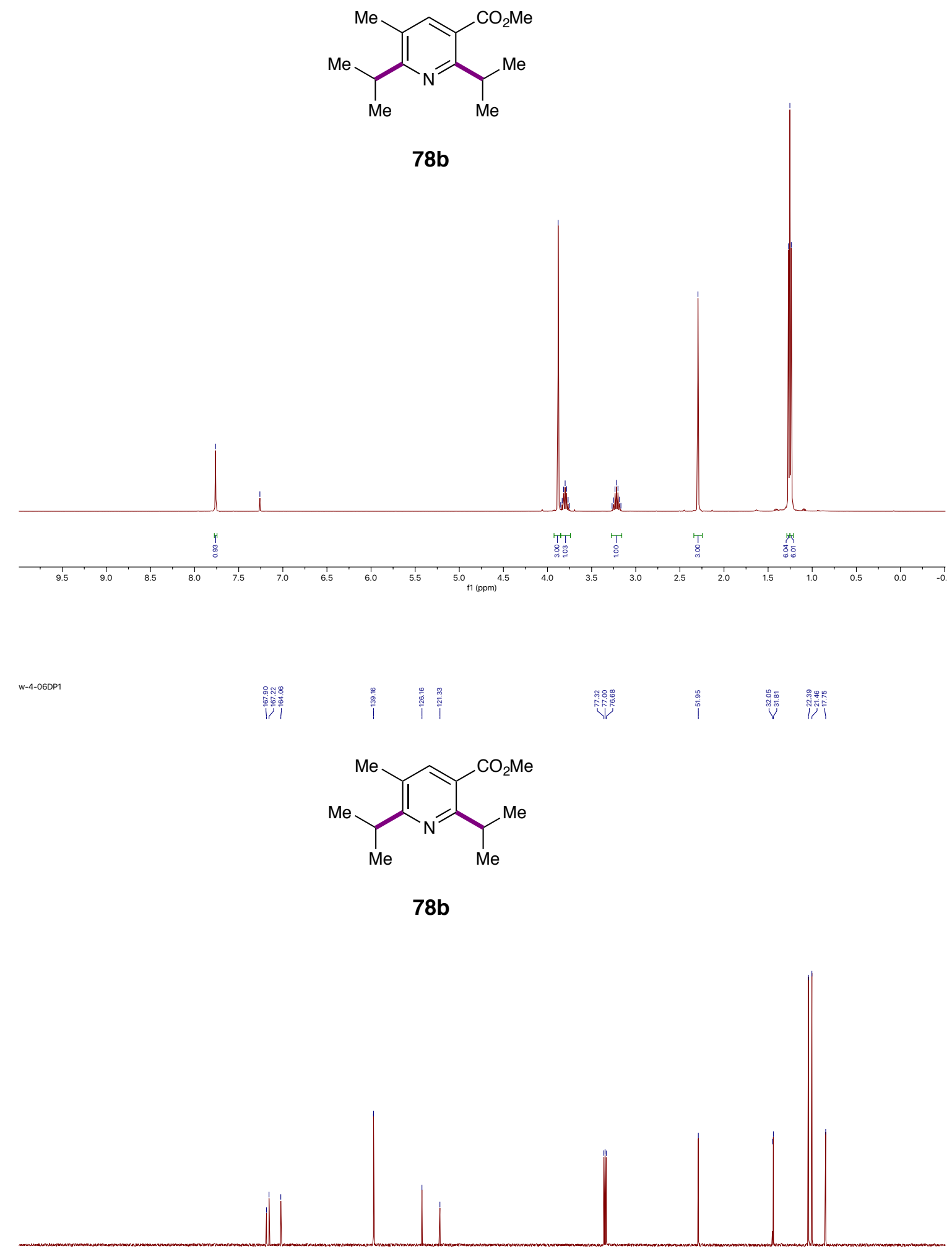

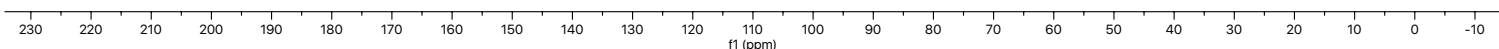




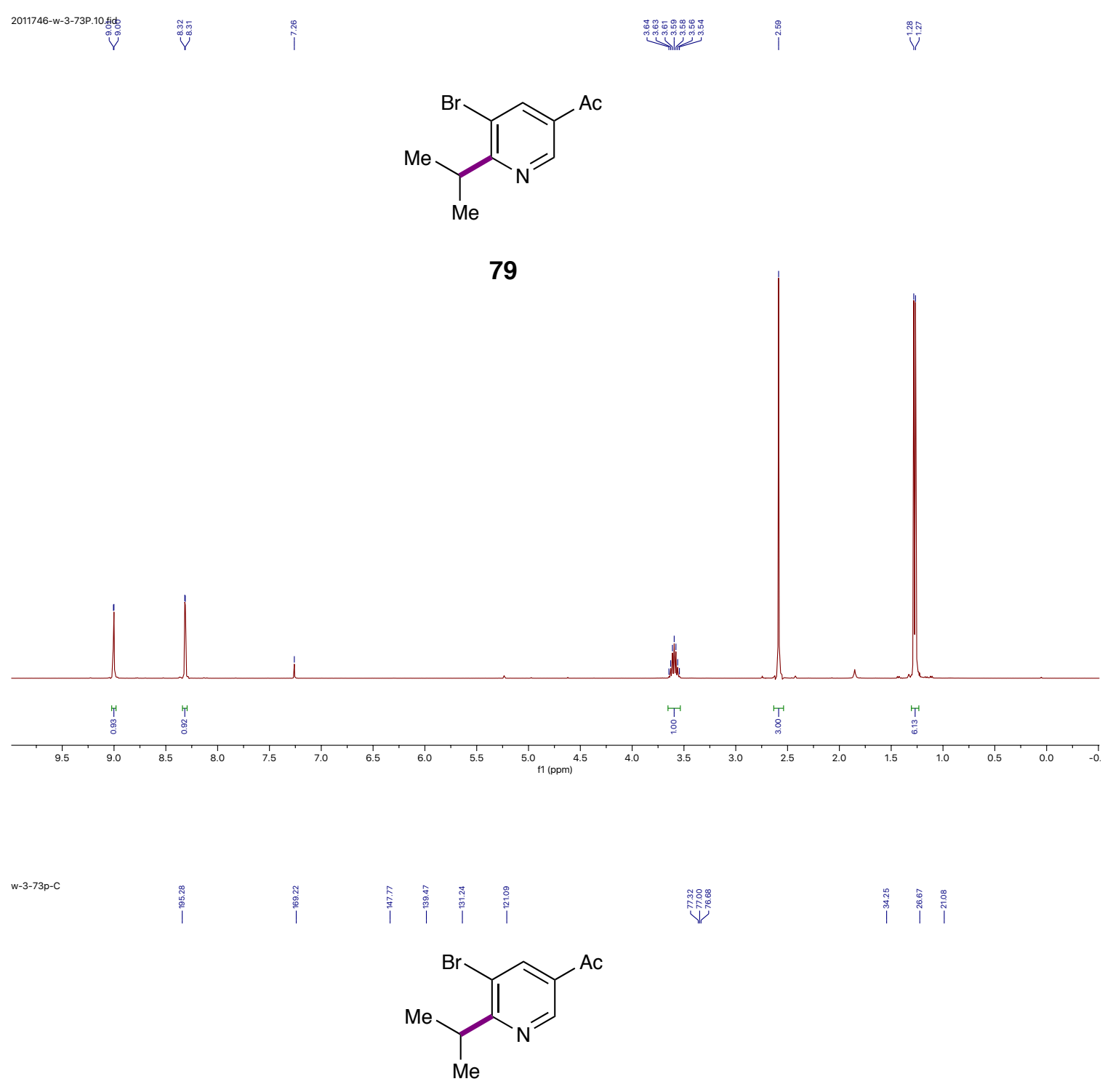

79

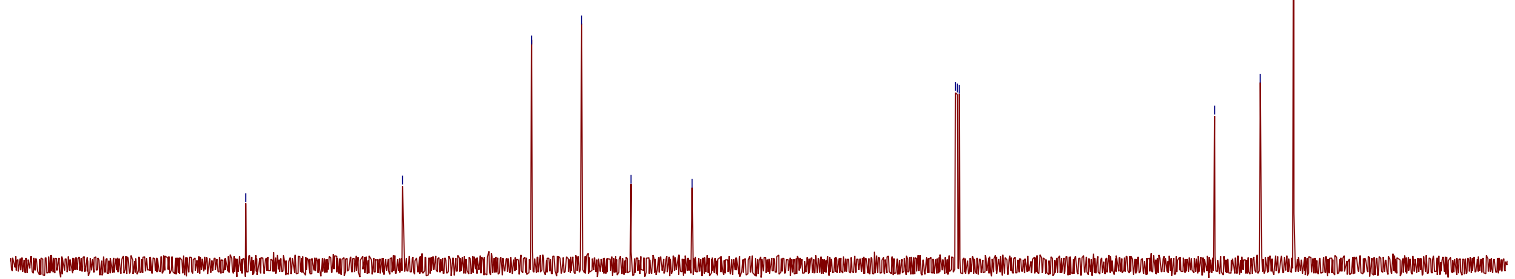

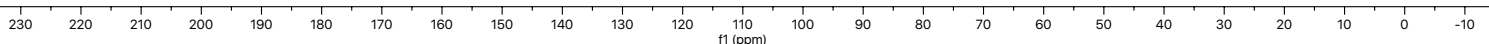



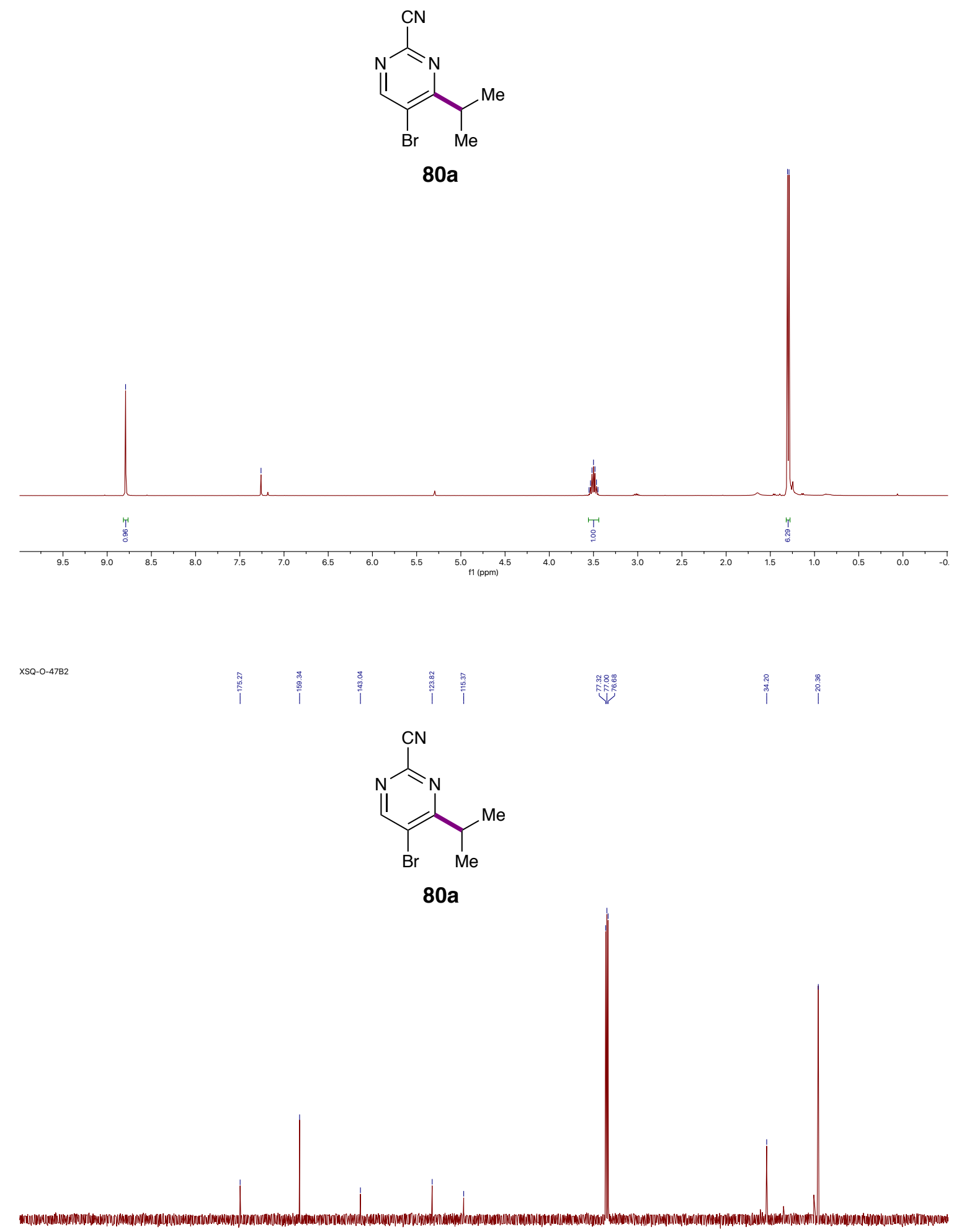

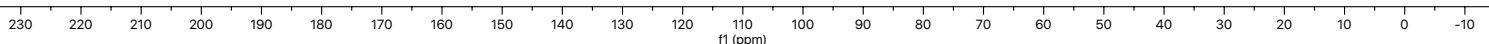



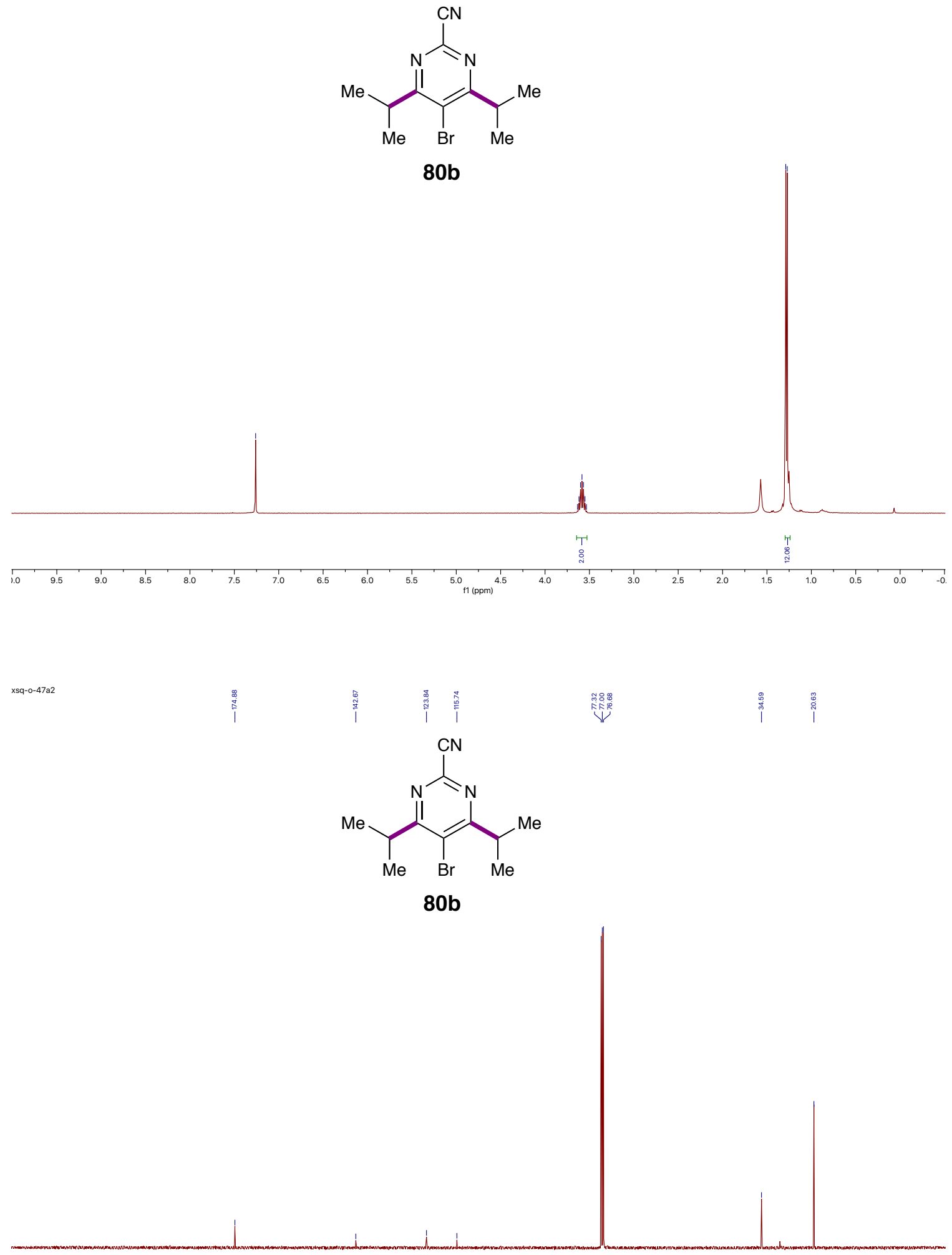

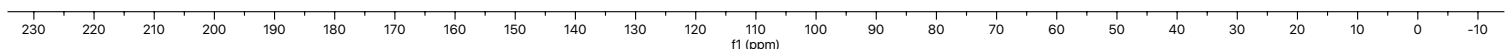




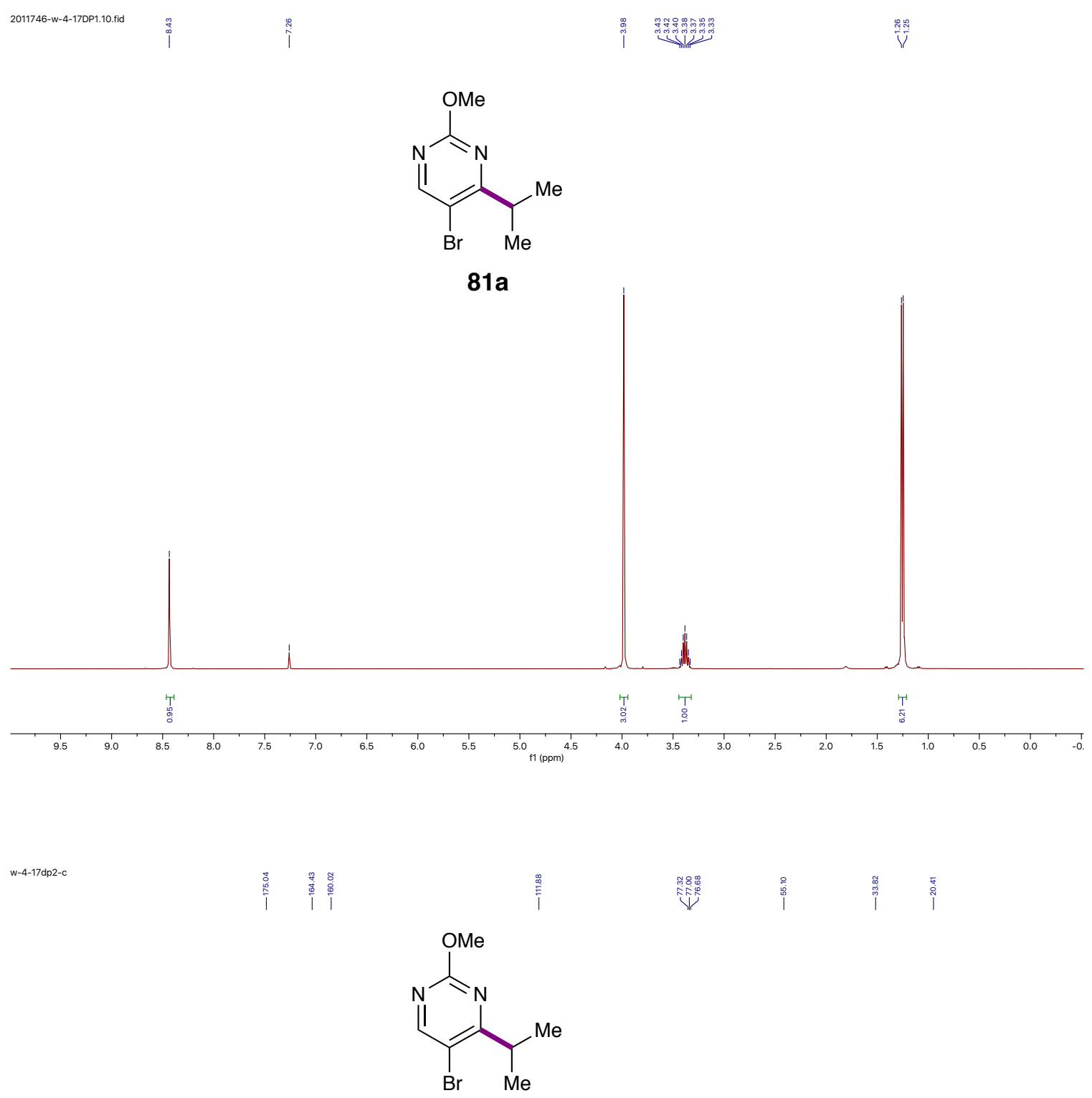

$81 a$

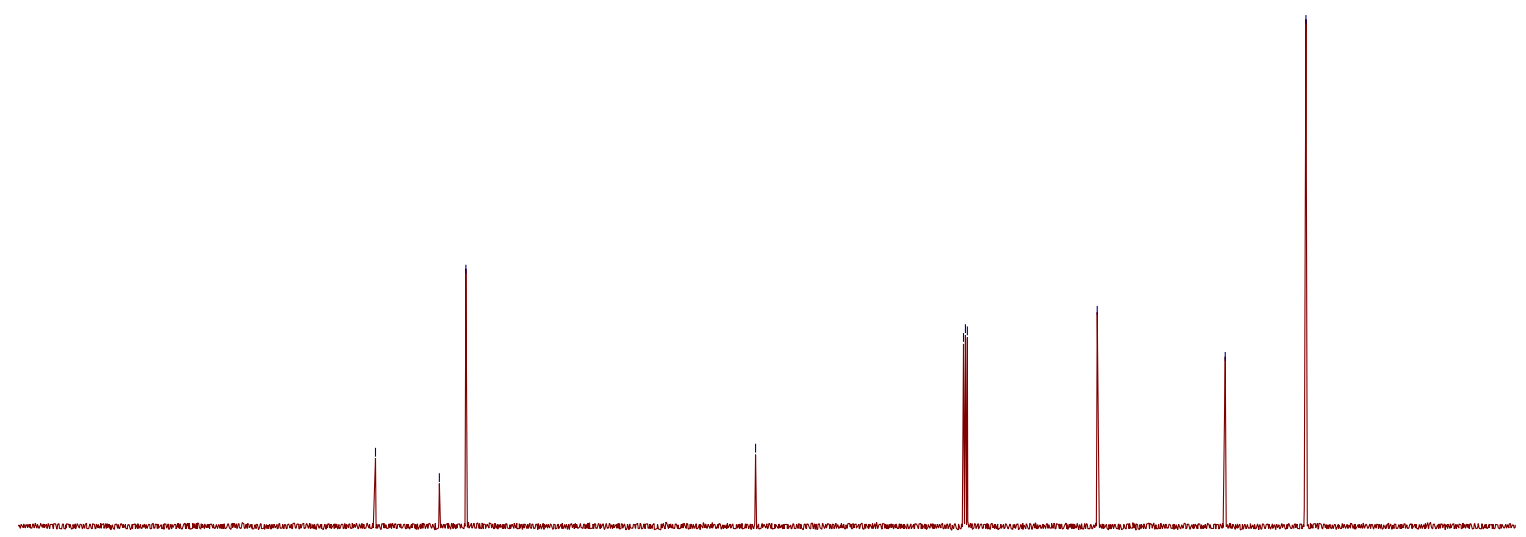

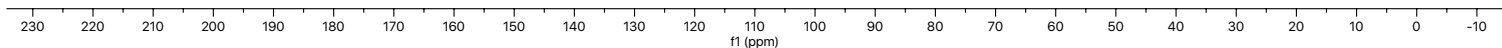



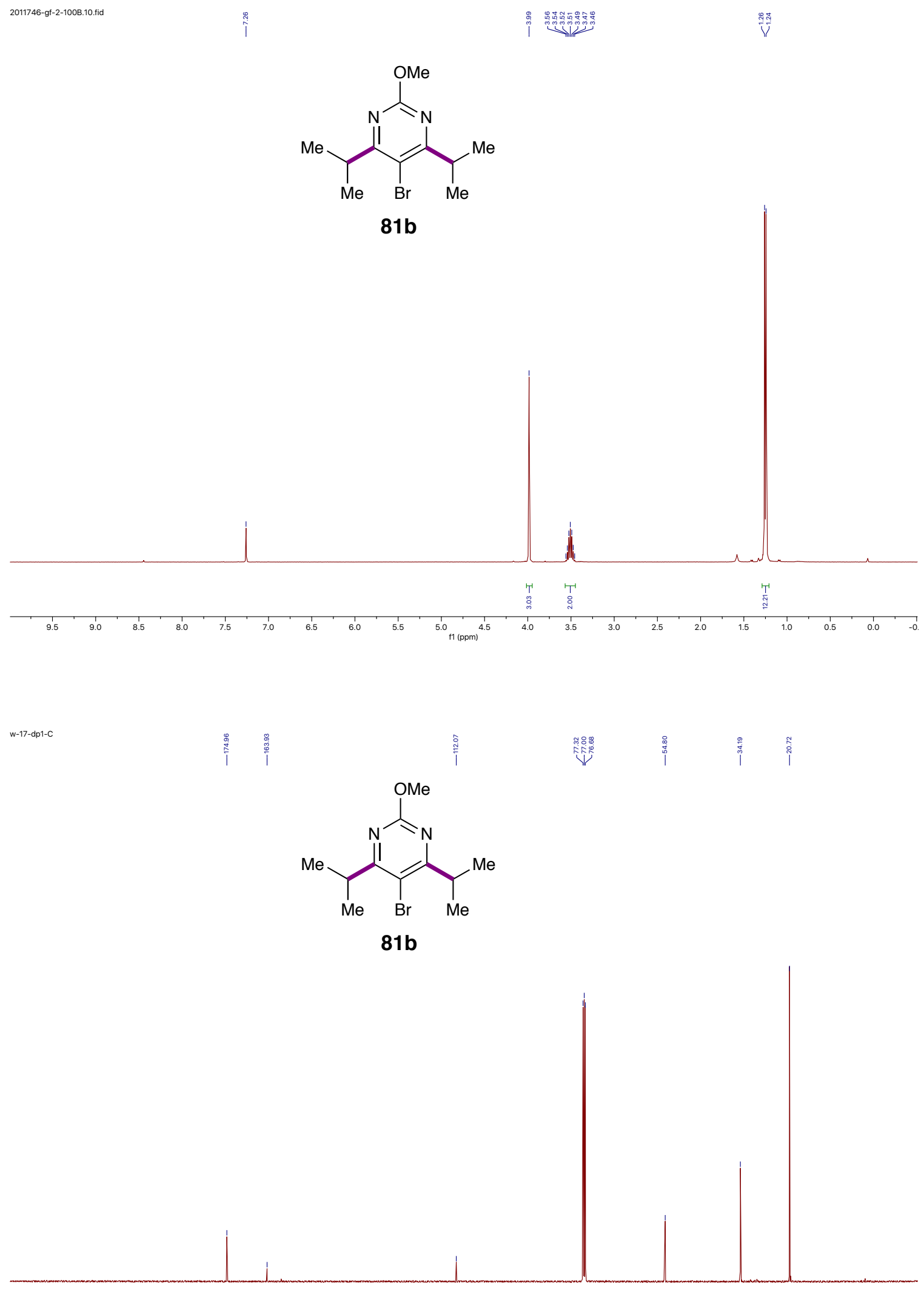

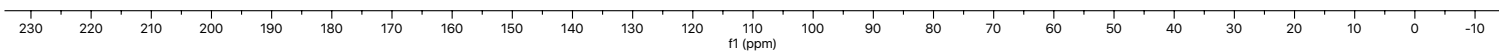



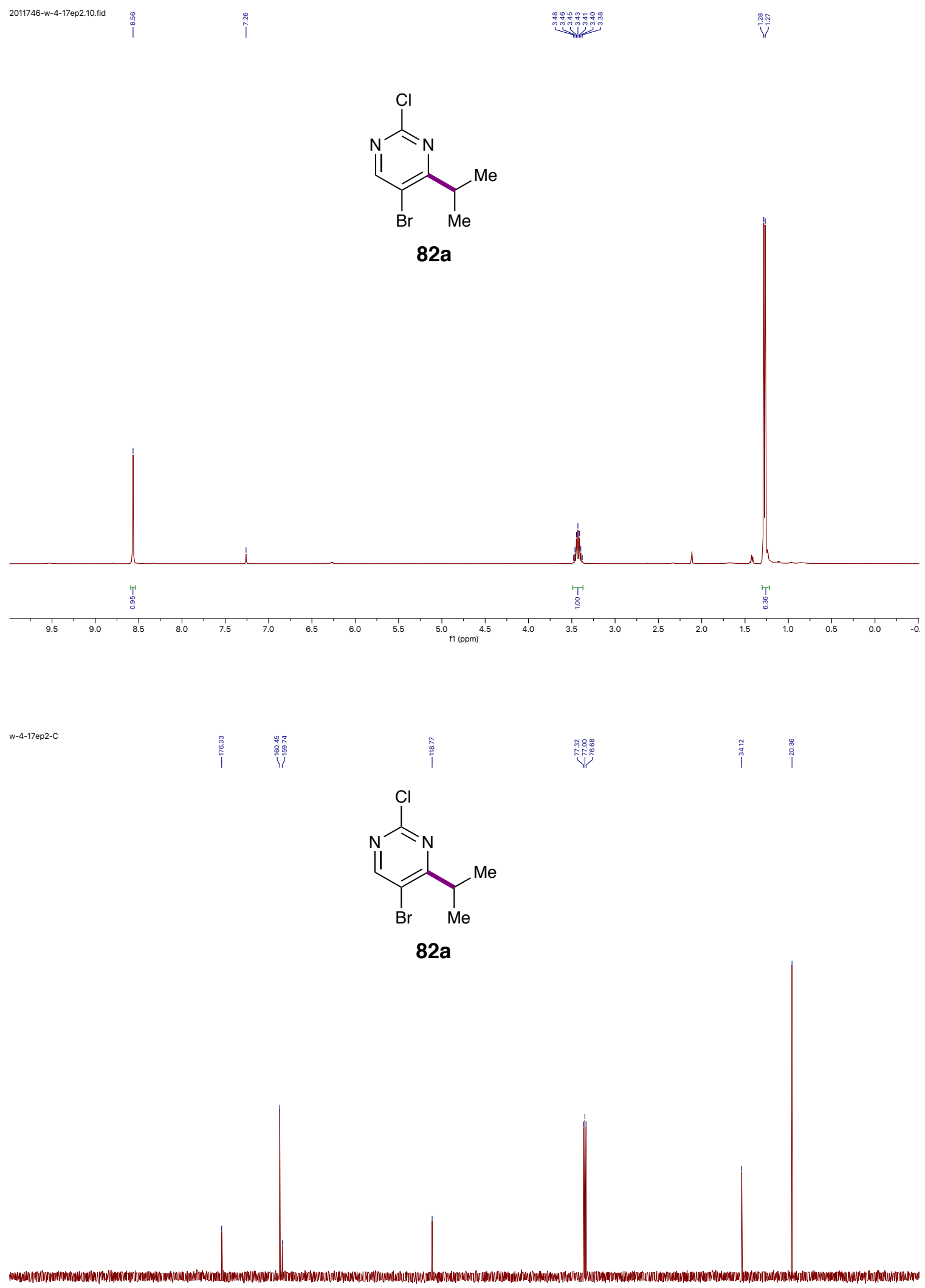

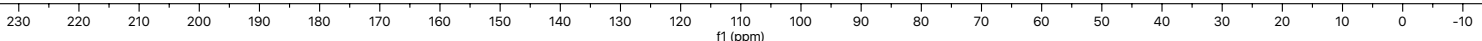



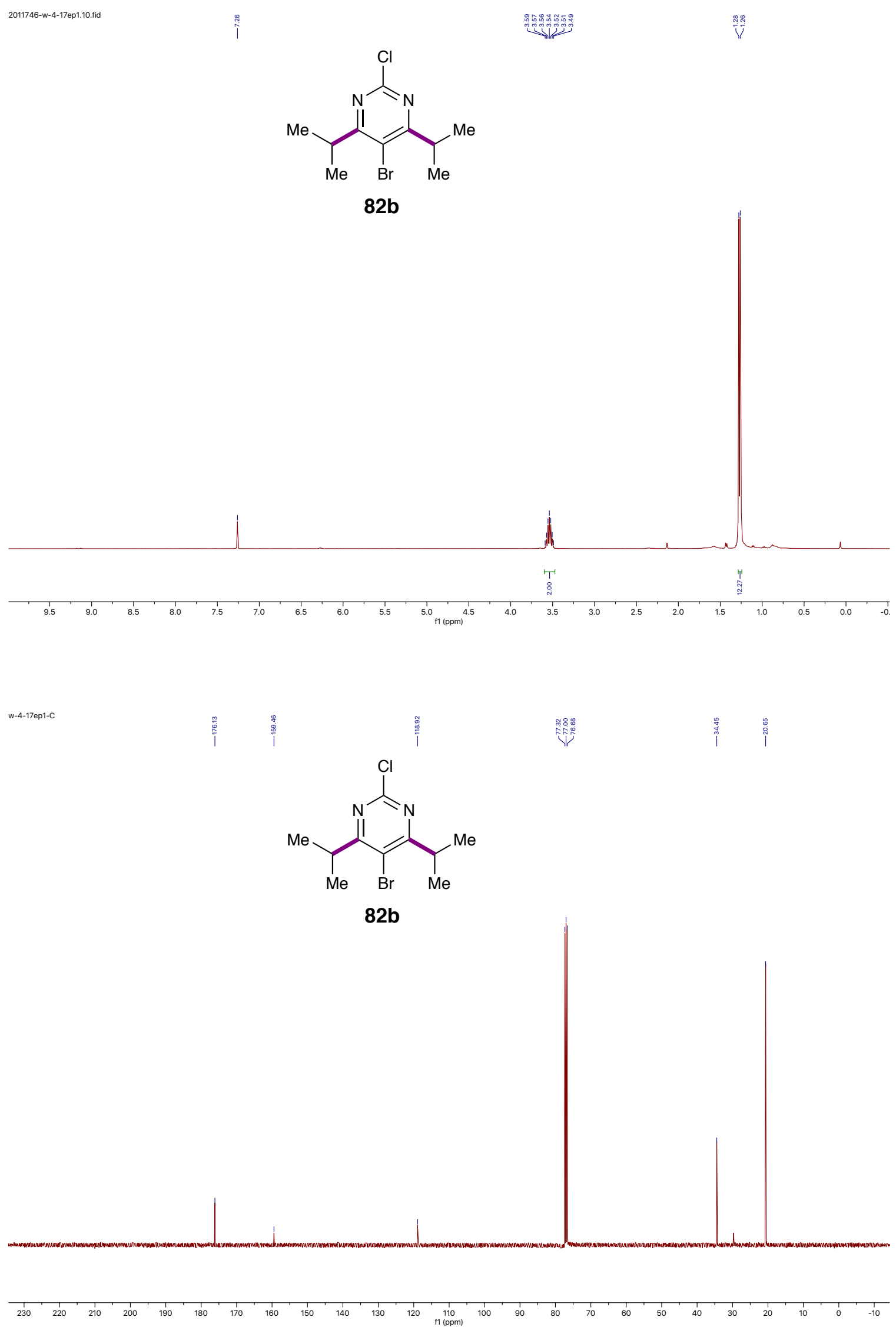


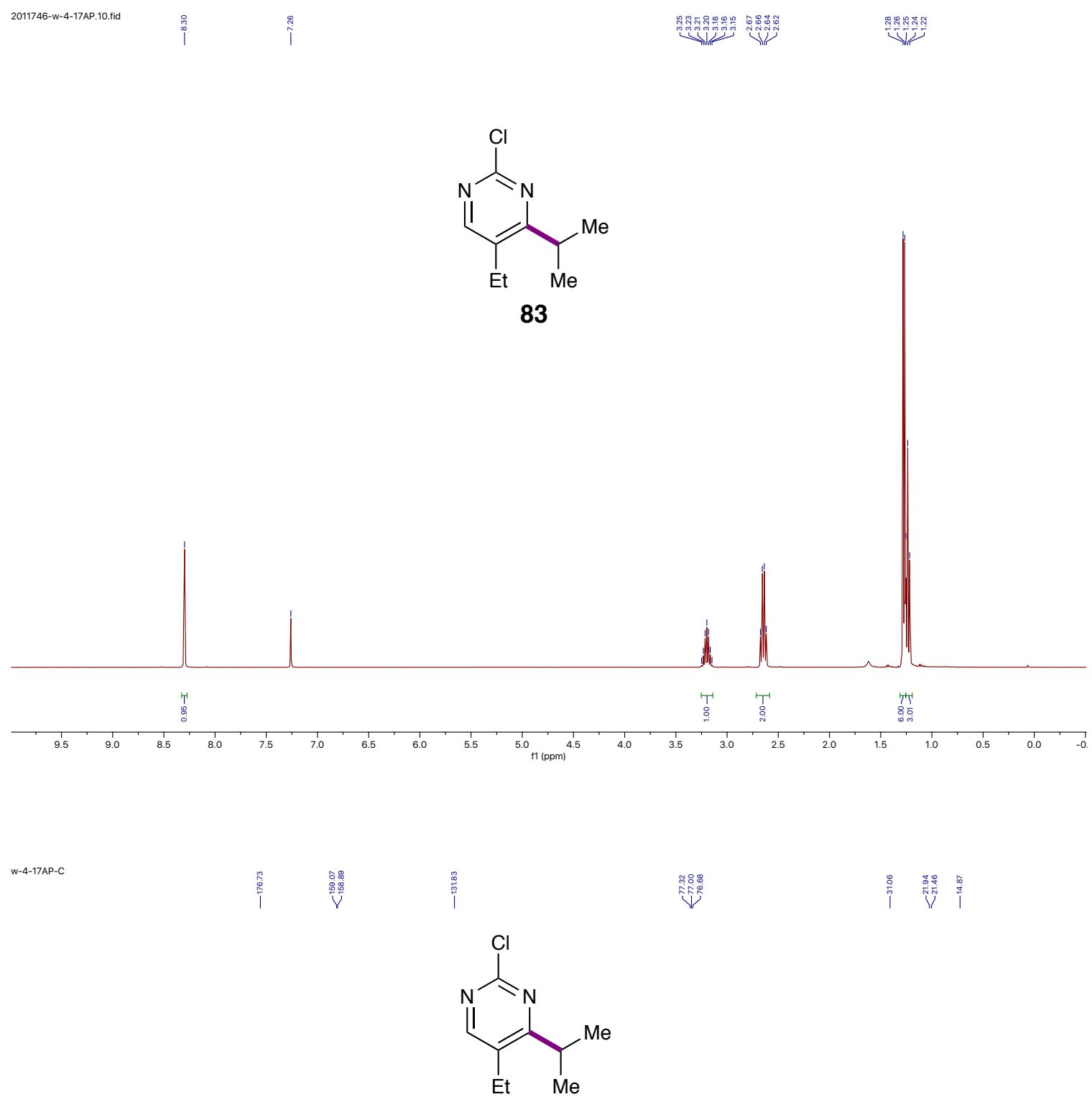

83

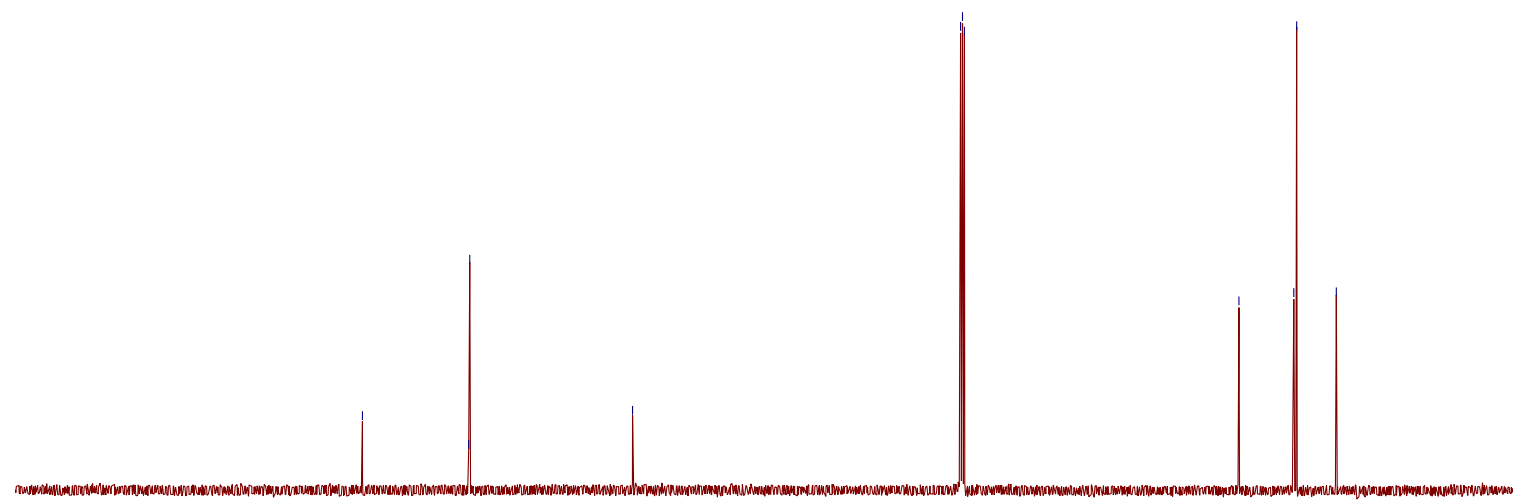

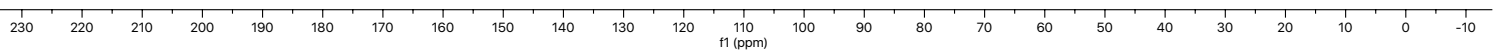




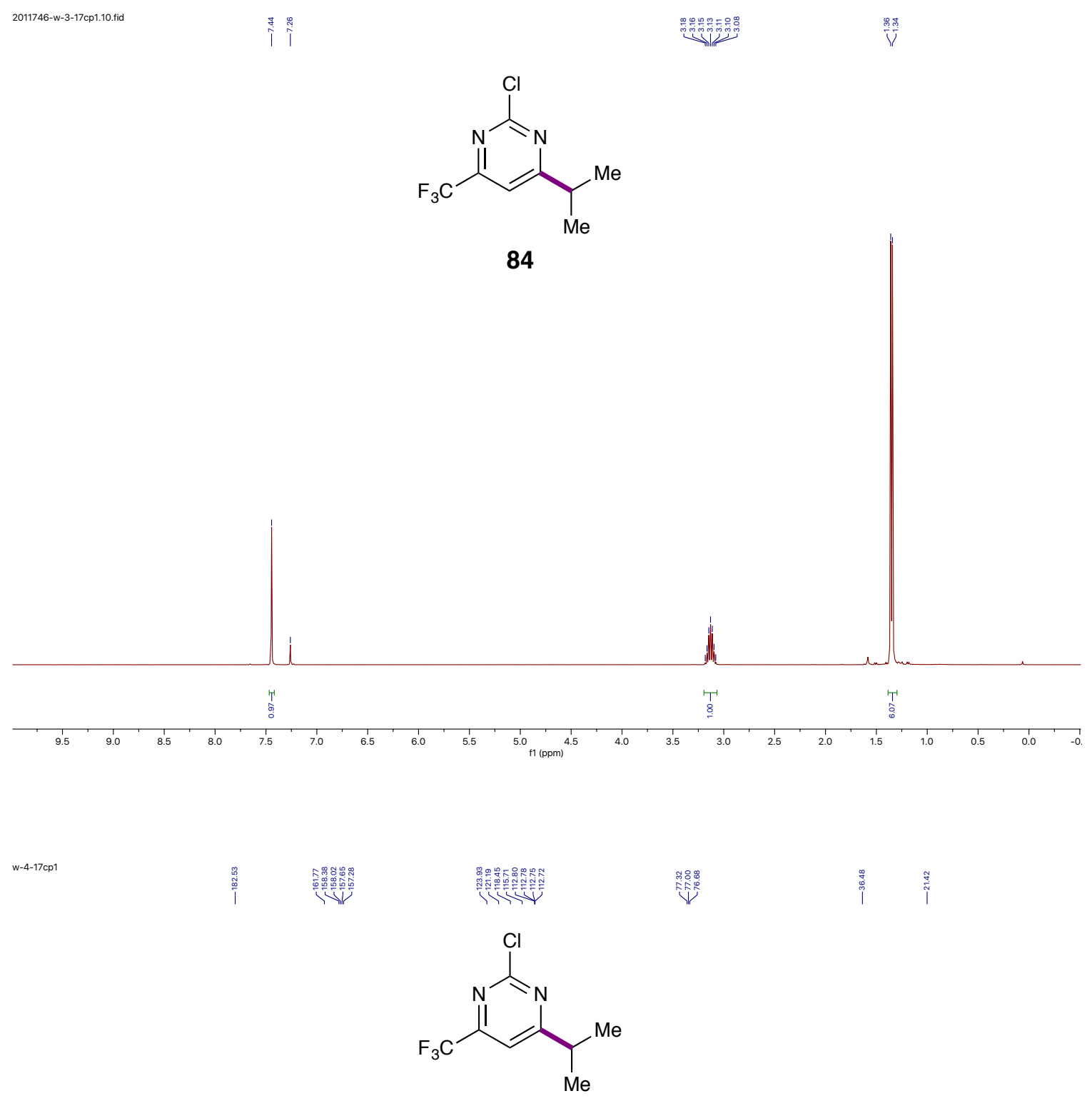

84

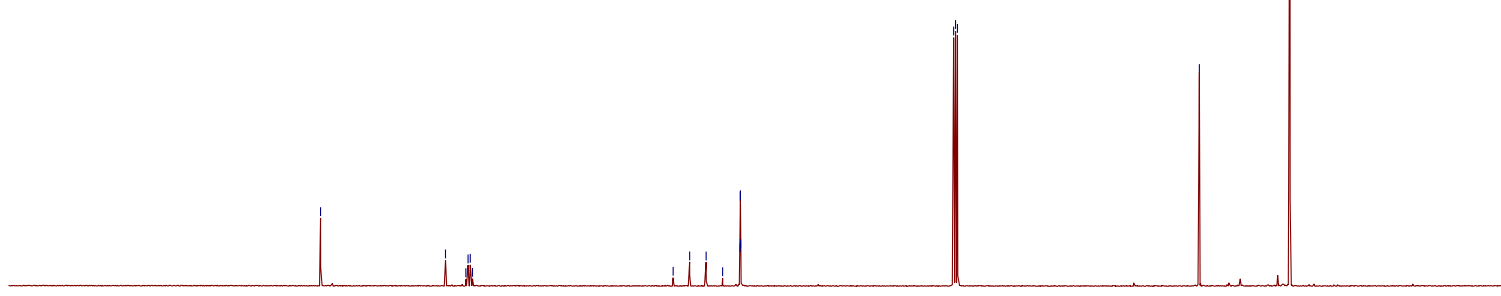

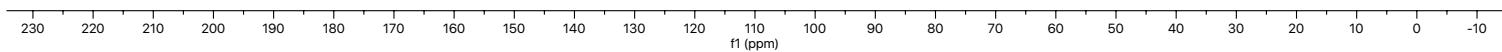



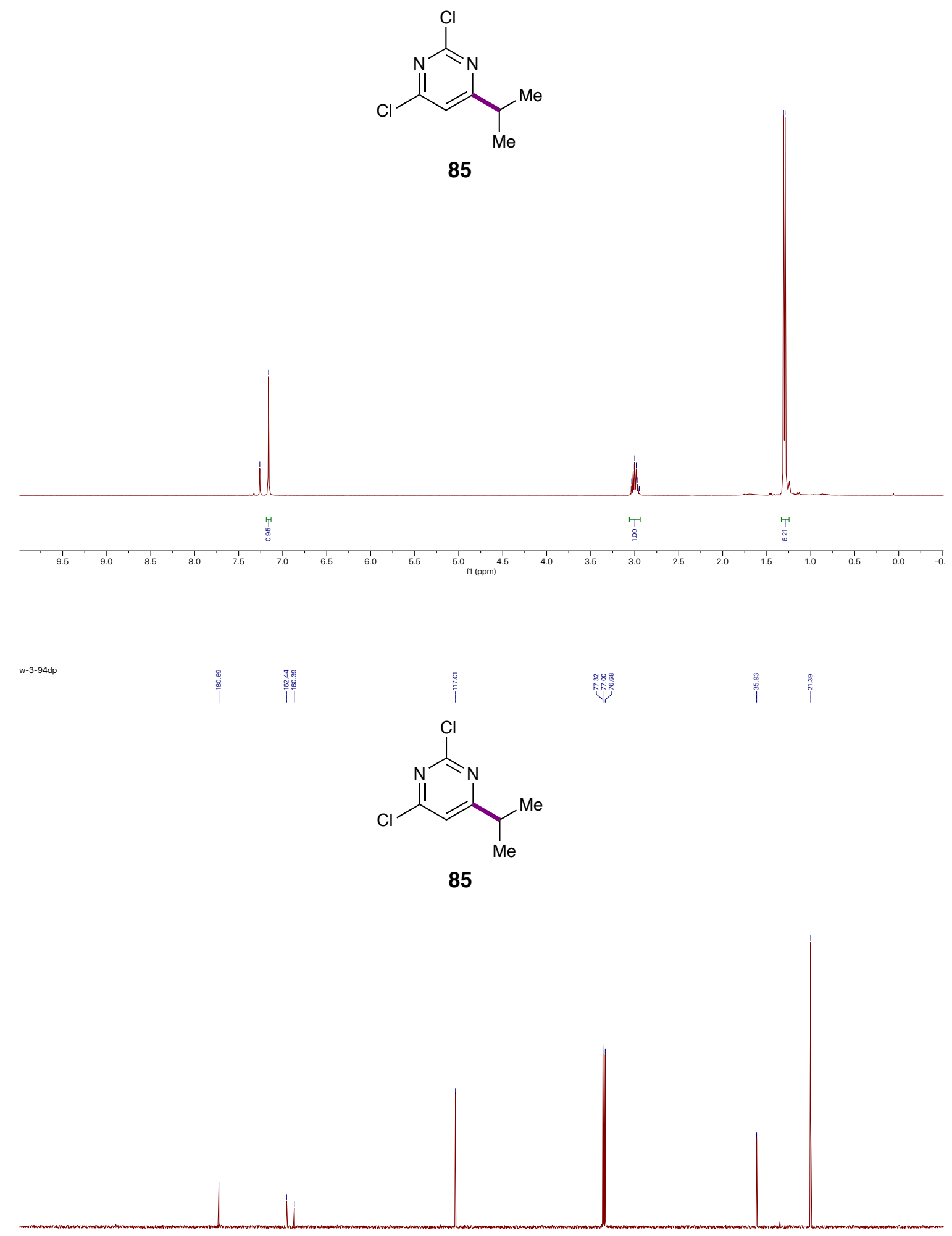

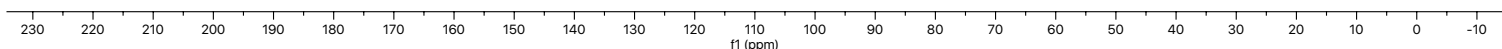


2011746-w-3-72P1.10.fid

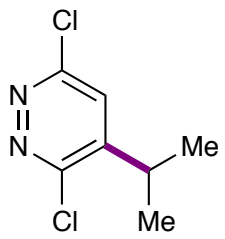

86

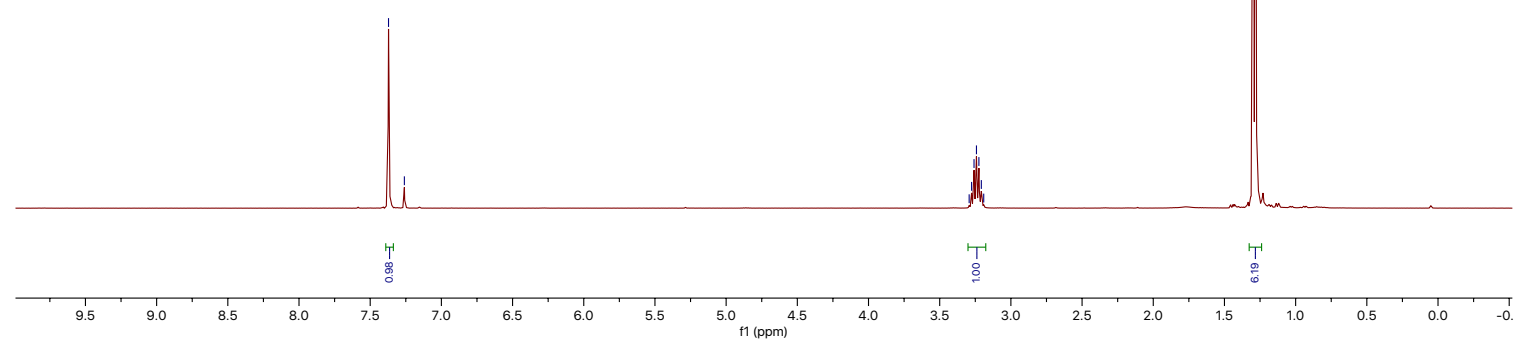

w-3-72p-c

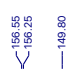
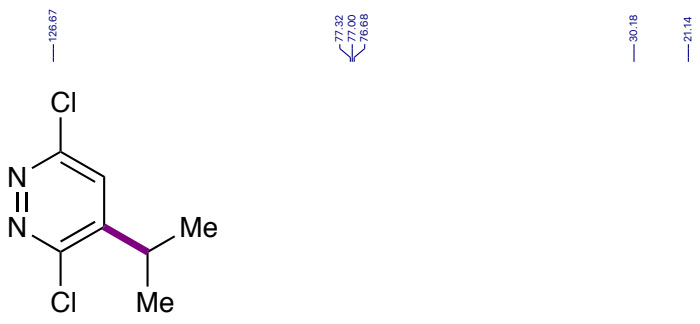

86

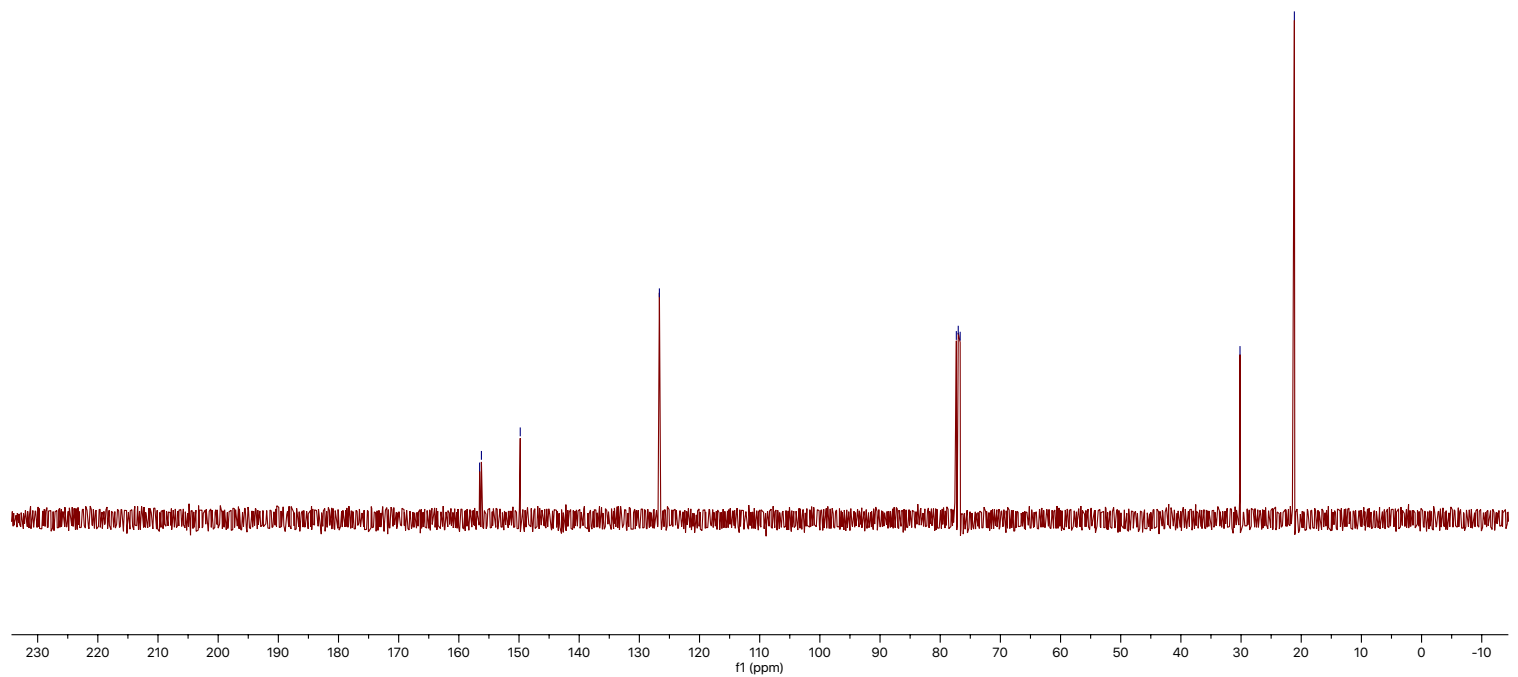




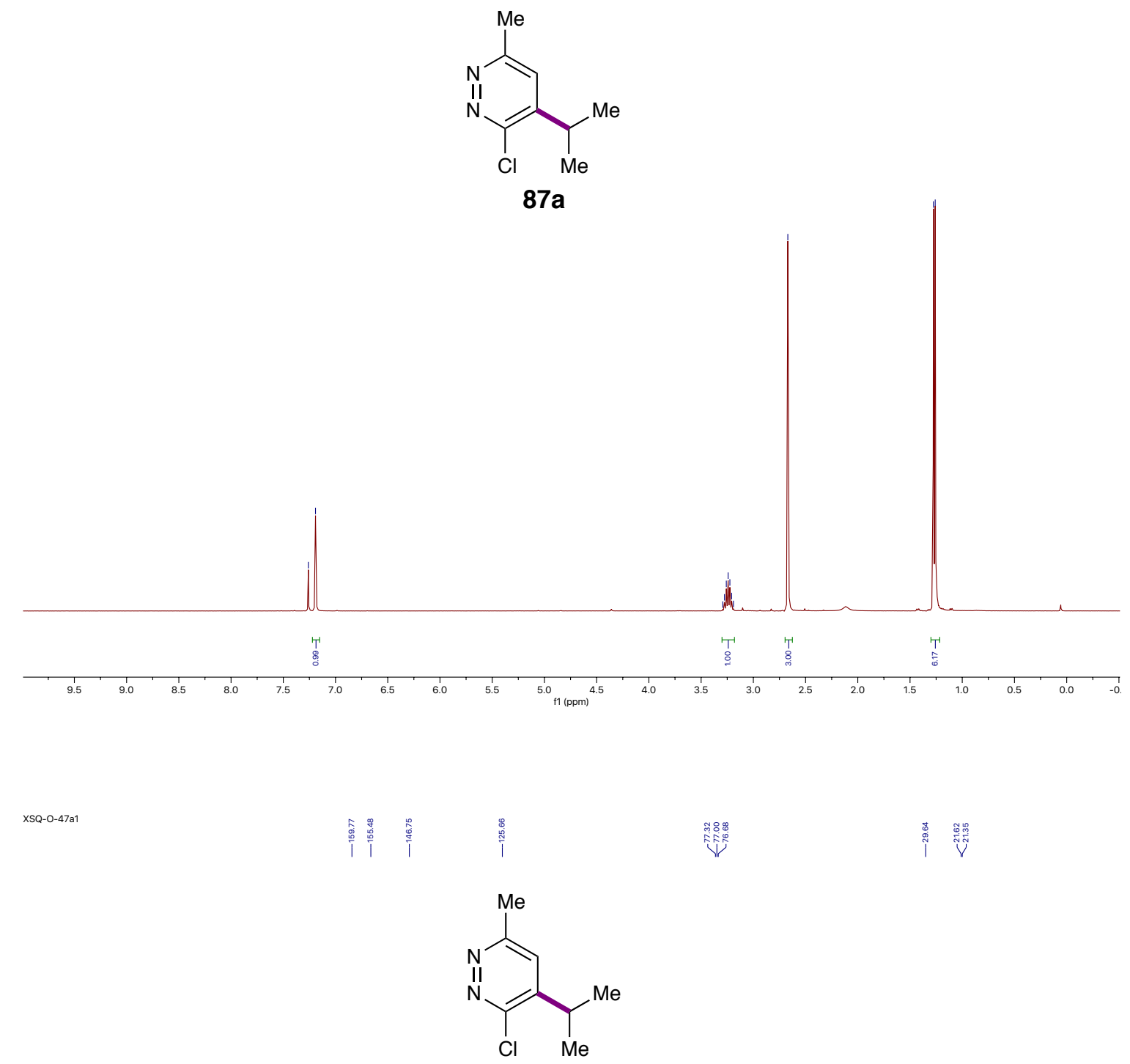

87a

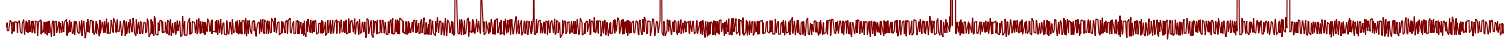

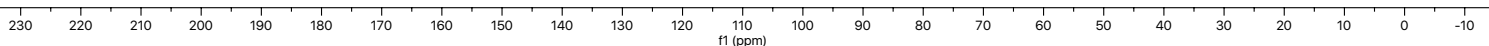



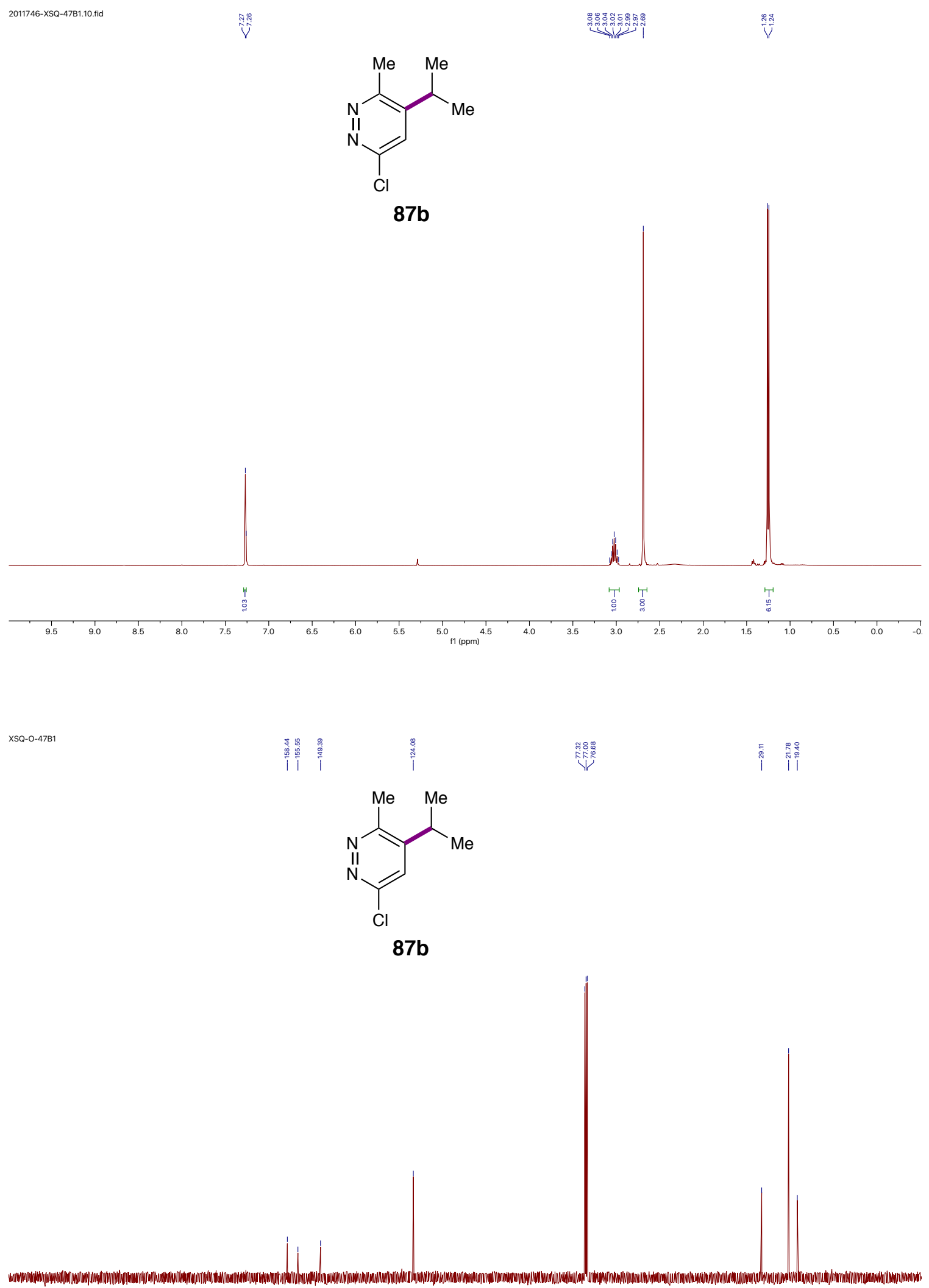

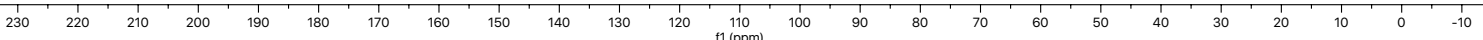




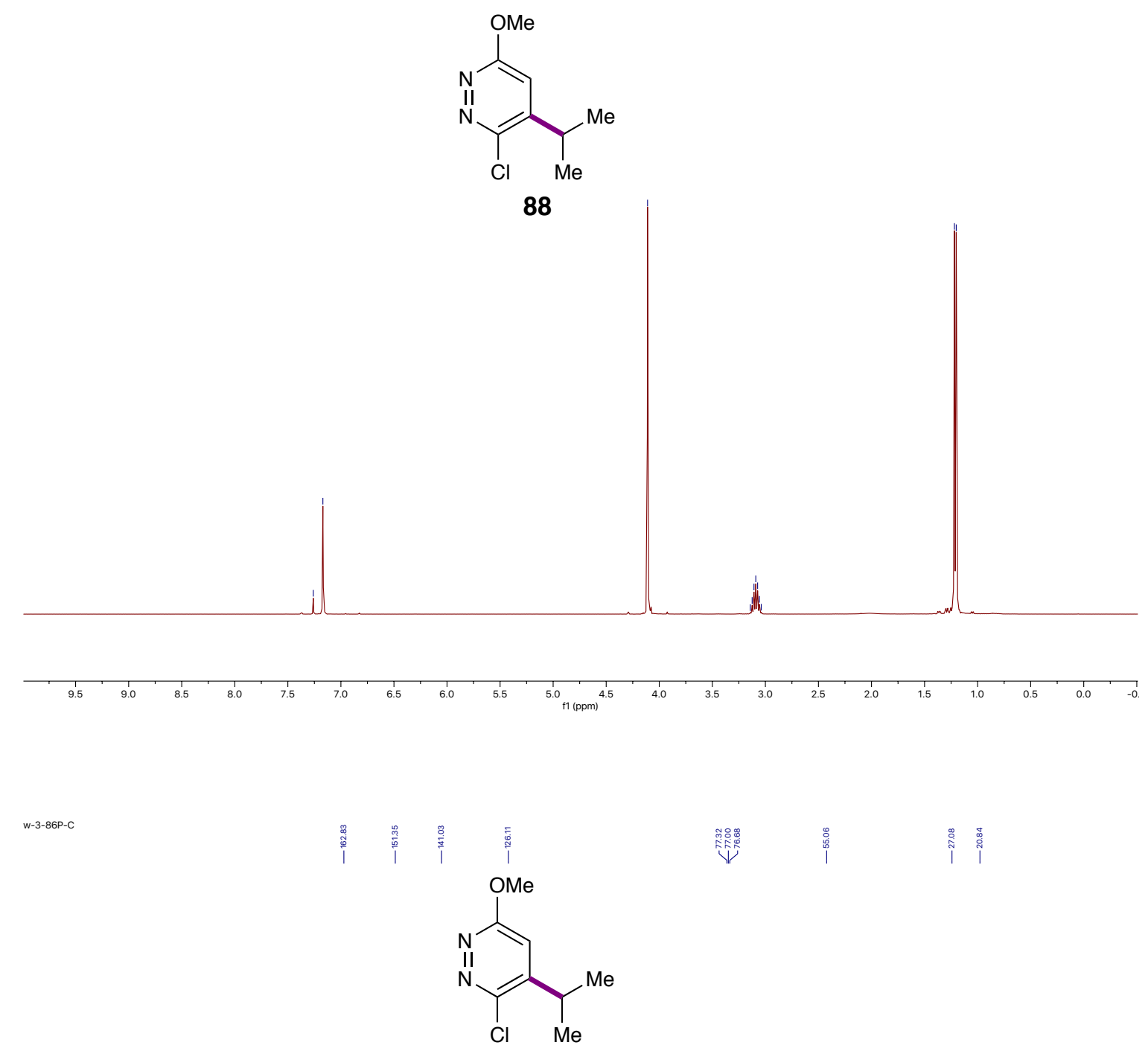

88

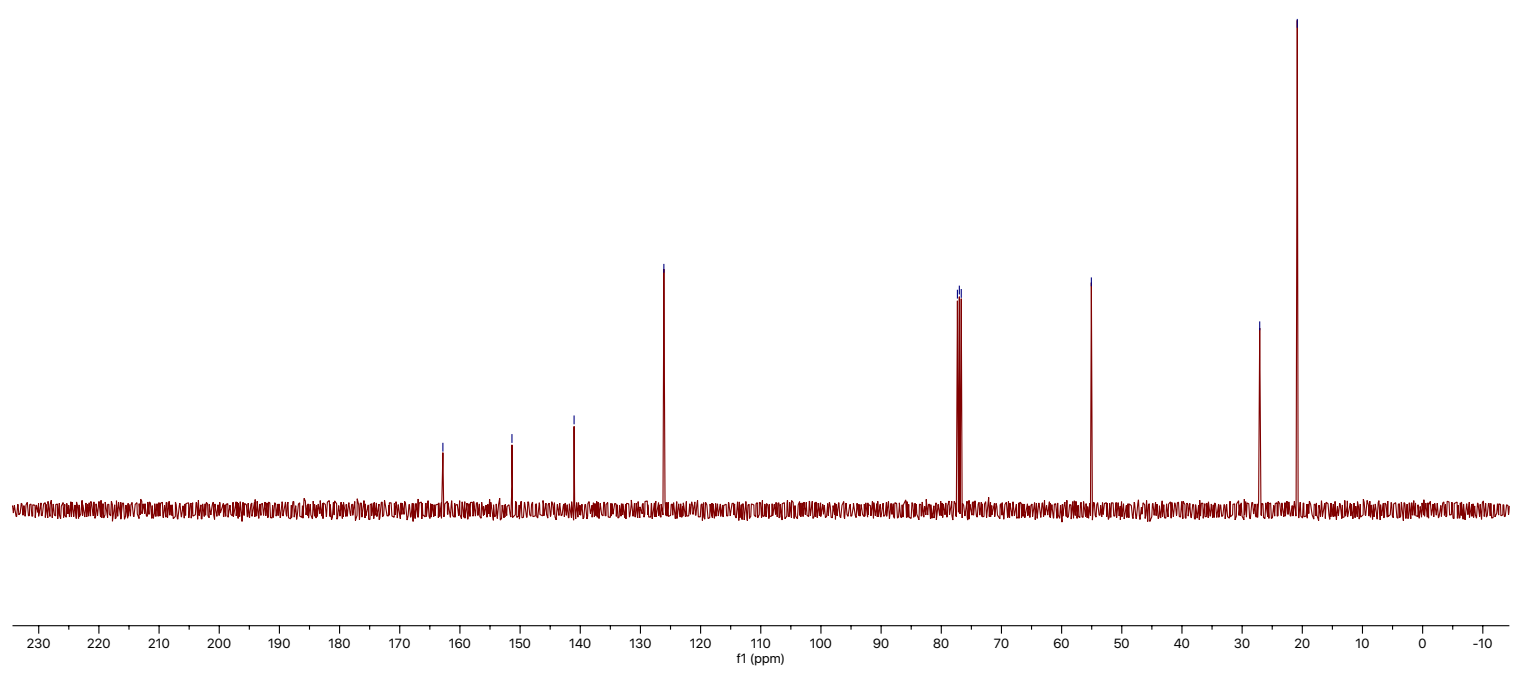




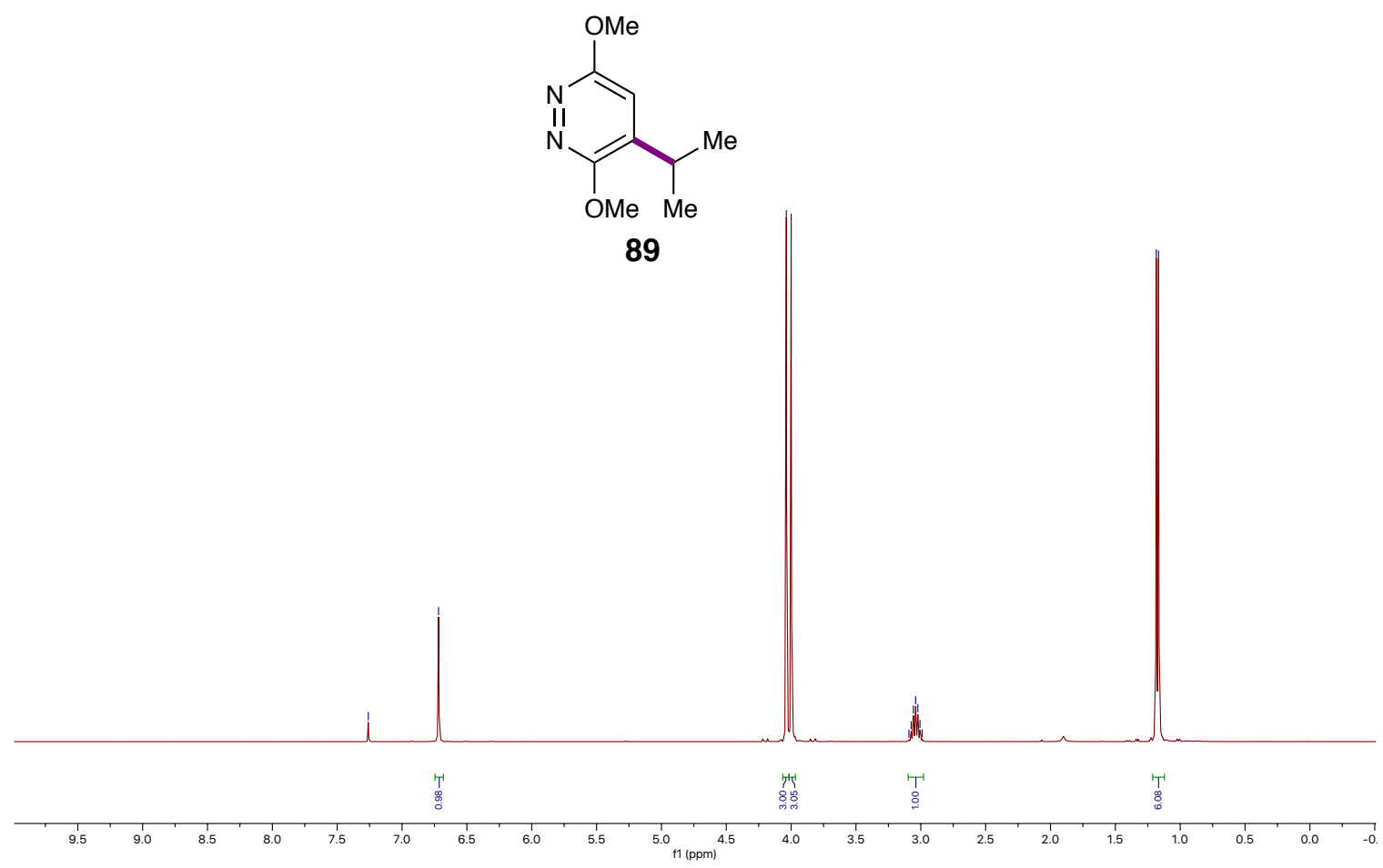

W-3-86DP-C

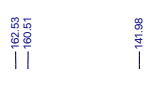
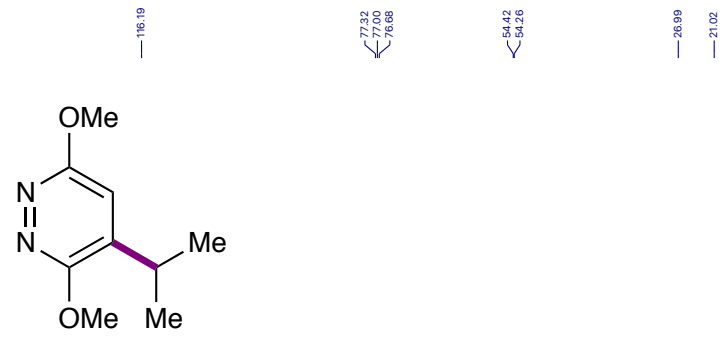

89

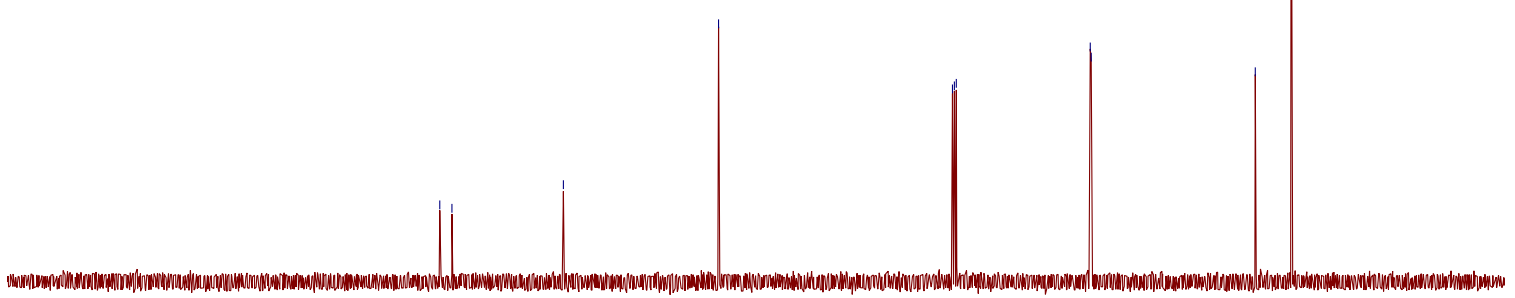

230

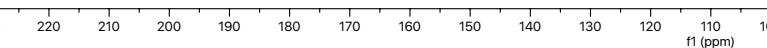




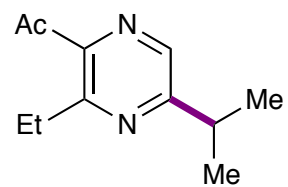

90

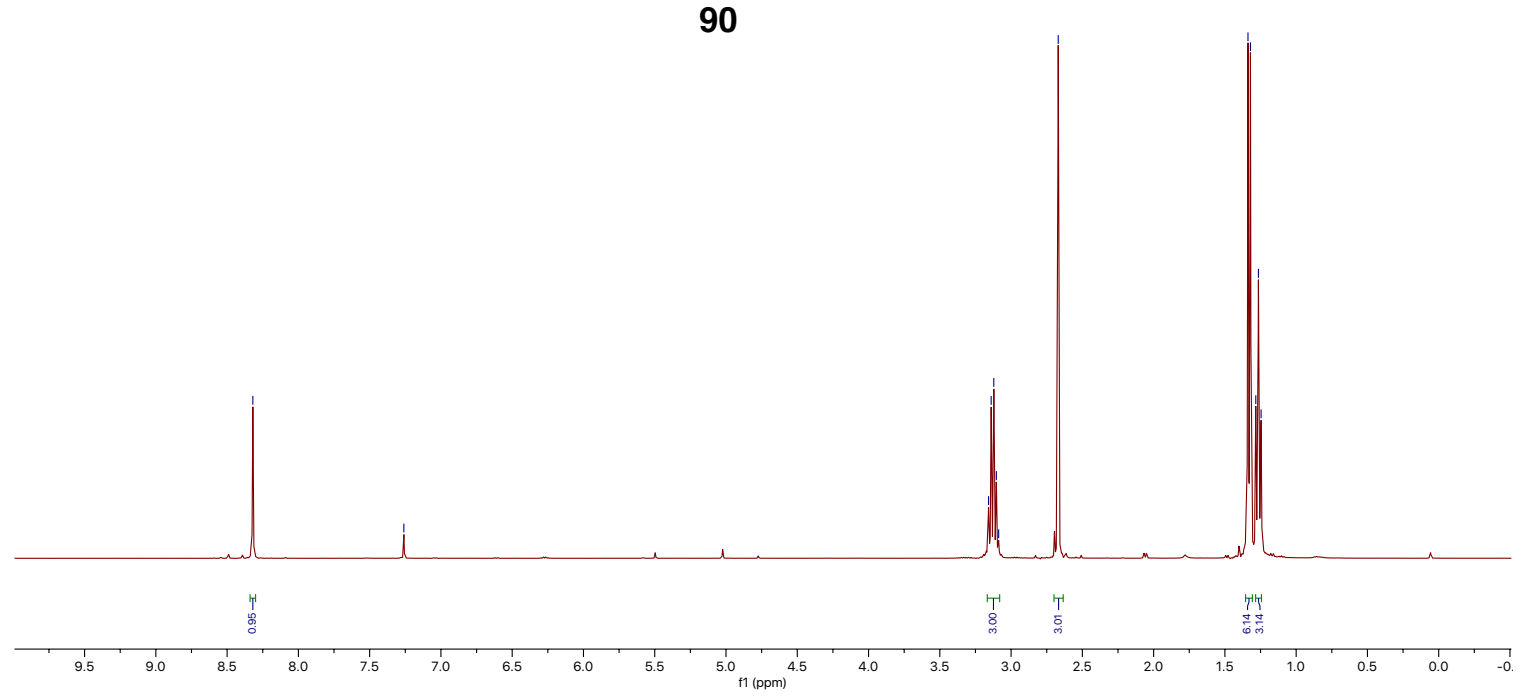

W-4-10GP

I

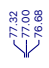

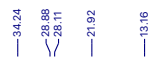

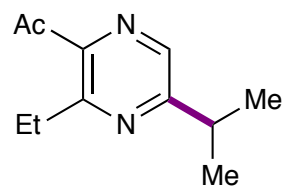

90

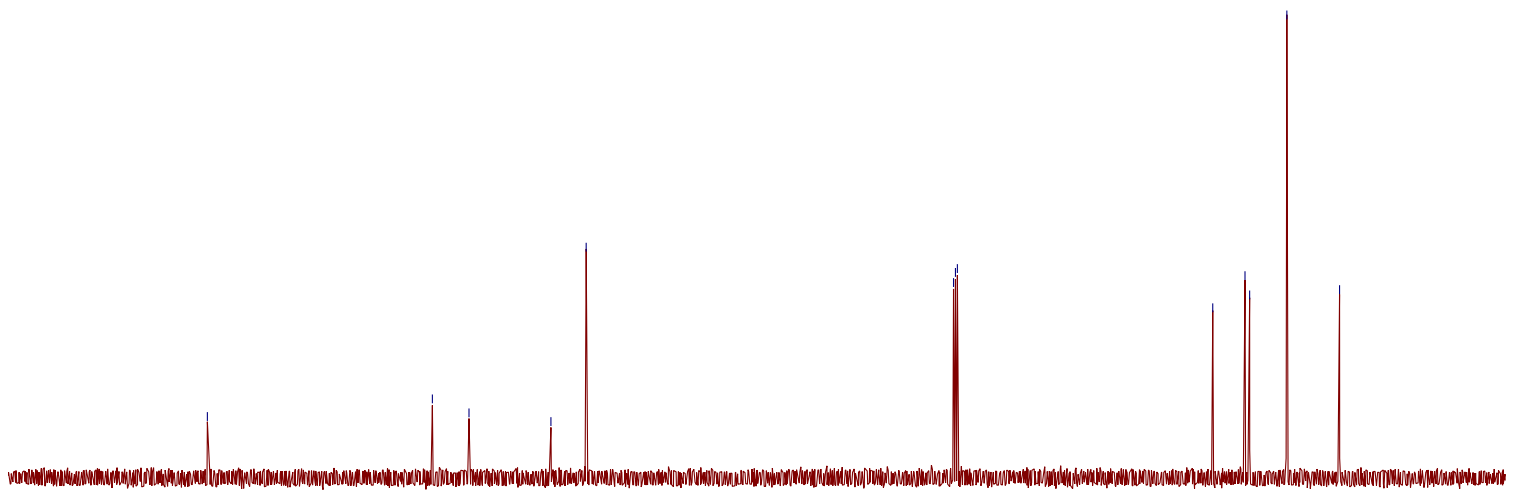

230

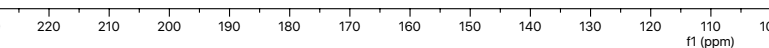




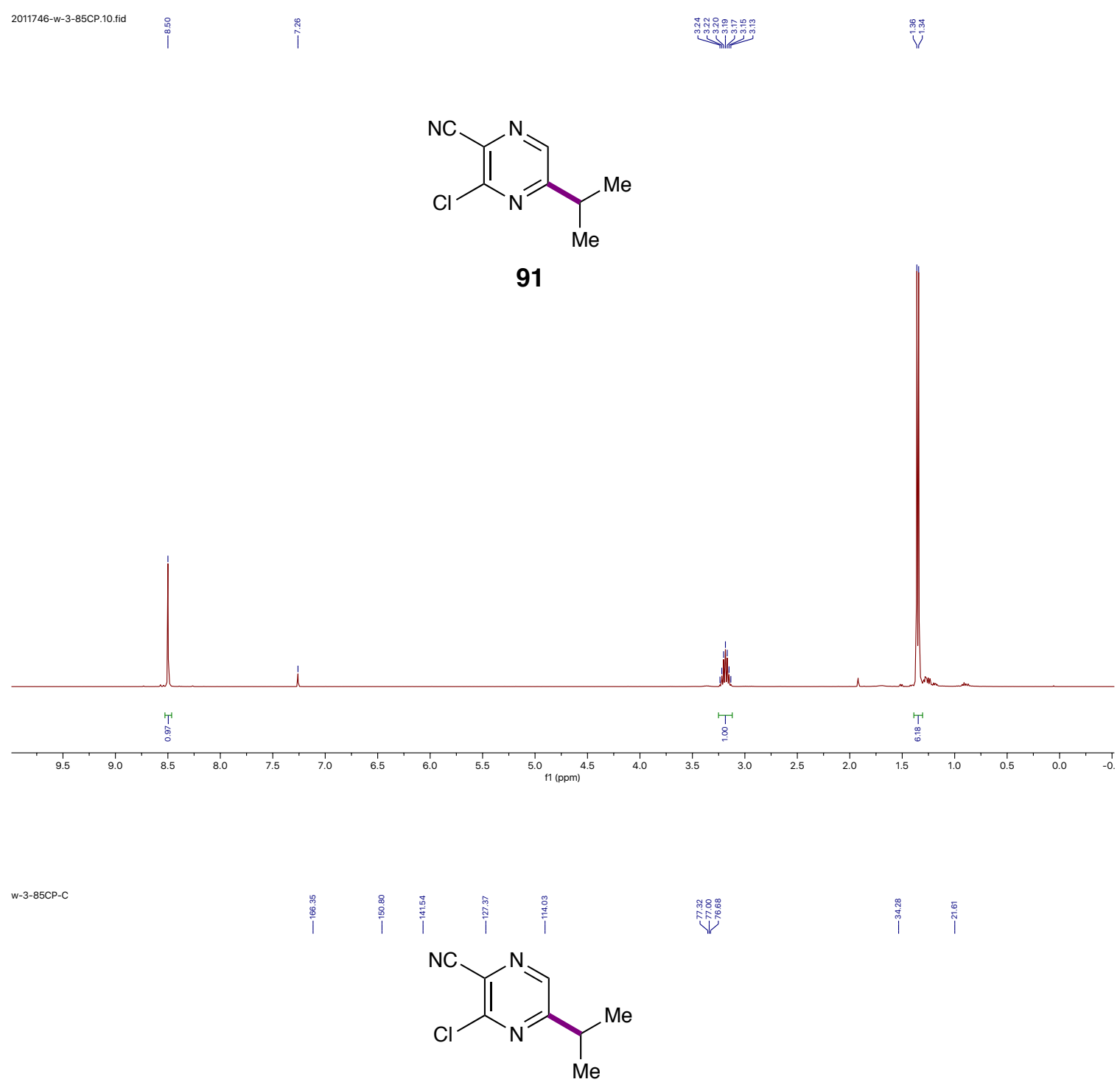

91

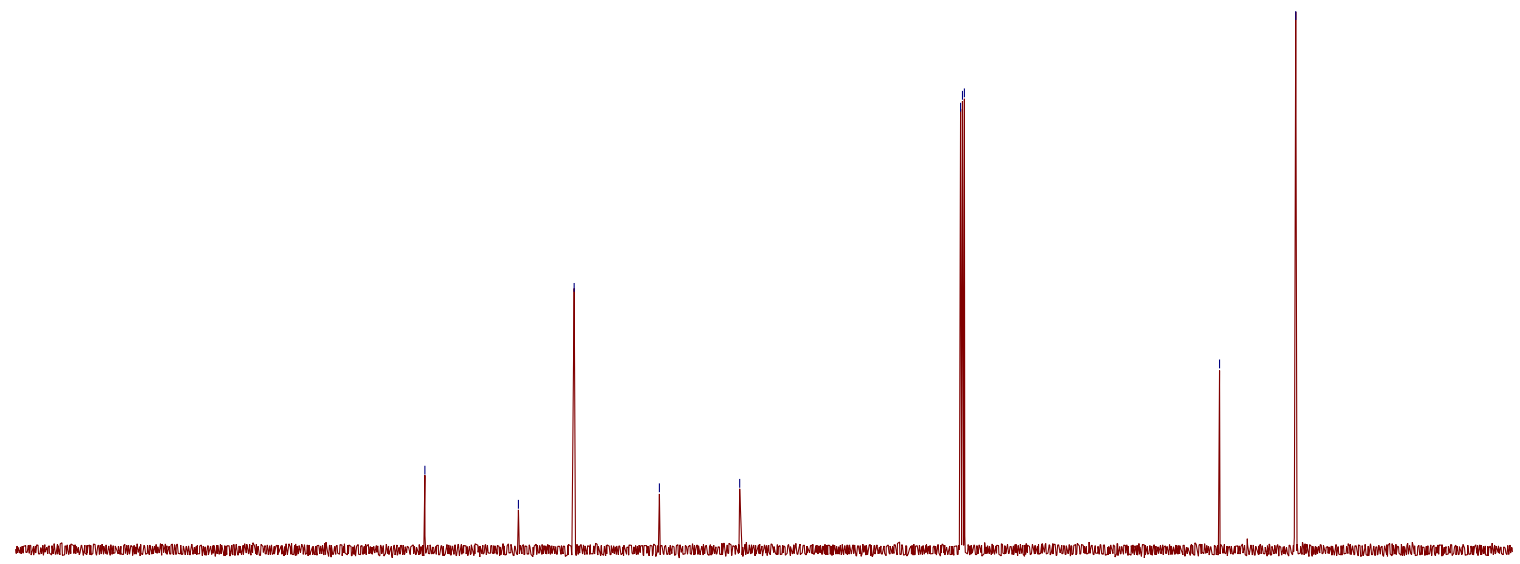

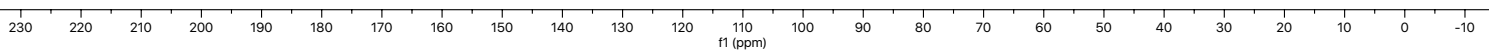


<smiles>CC(C)c1c(C=O)sc2ccccc12</smiles>

92

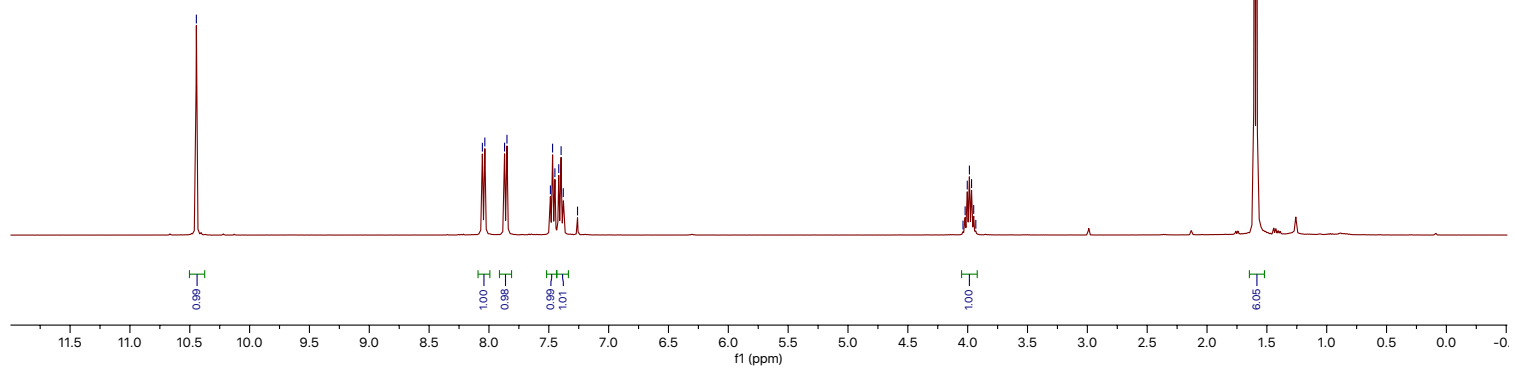

w-3-91Dp-C

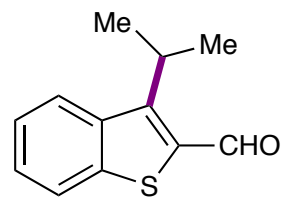

92

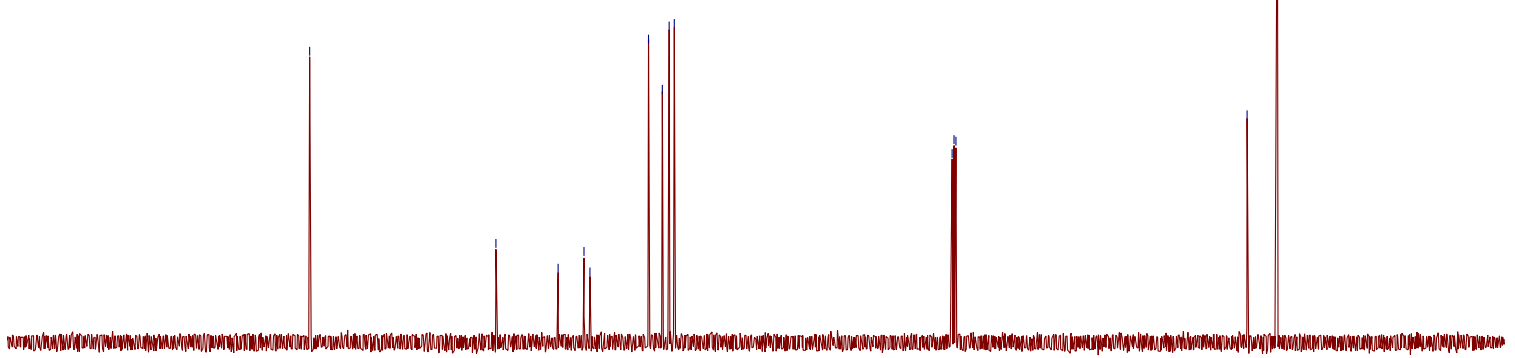

230

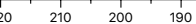

$\begin{array}{llll}180 & 170 & 160 & 150\end{array}$

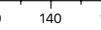

f1 (ppons 
2011746-w-3-90HP-re.10.fid

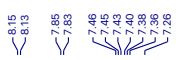

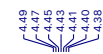<smiles>CC(C)c1c(C#N)sc2ccccc12</smiles>

93

-w-3-88dp-C

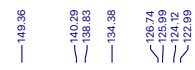

路影

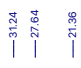<smiles>Cc1sc2ccccc2c1C(C)C</smiles>

93

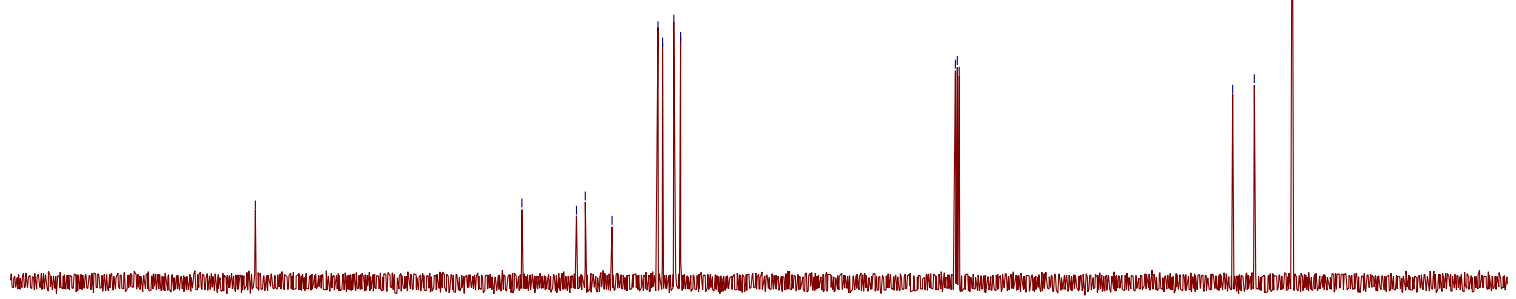

230

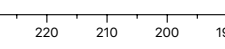

110
$\mathrm{f1}(\mathrm{pp})$ 


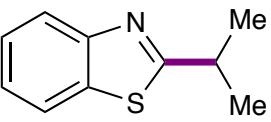

94

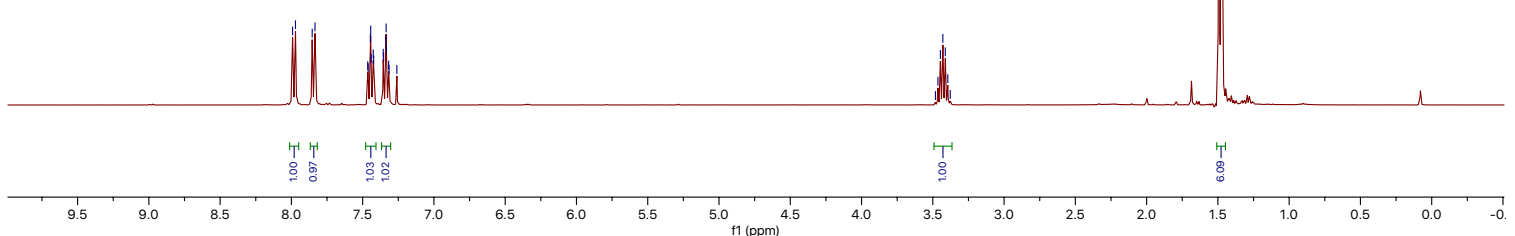

w-3-82pc
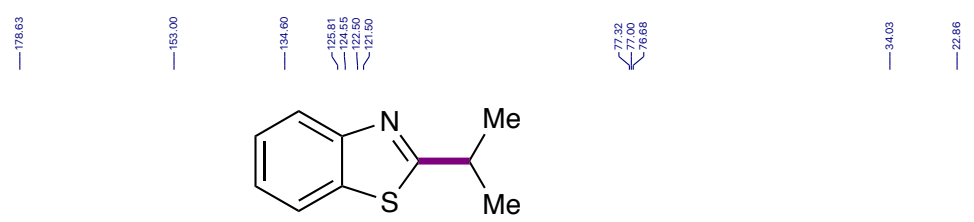

94 


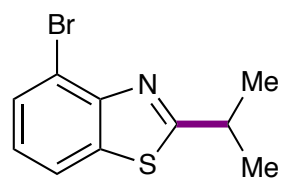

95

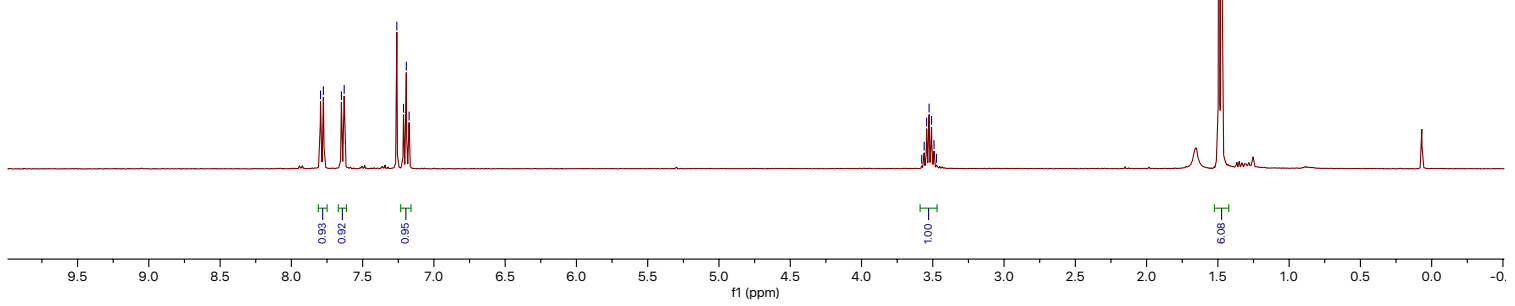

w-3-80p-C
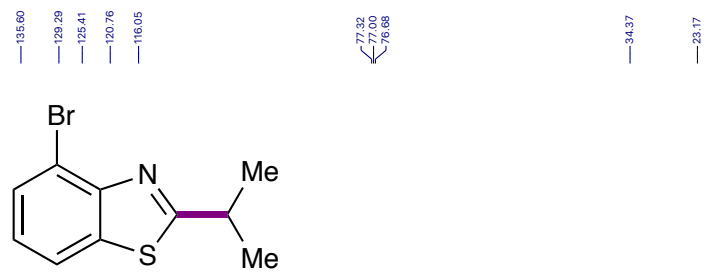

95
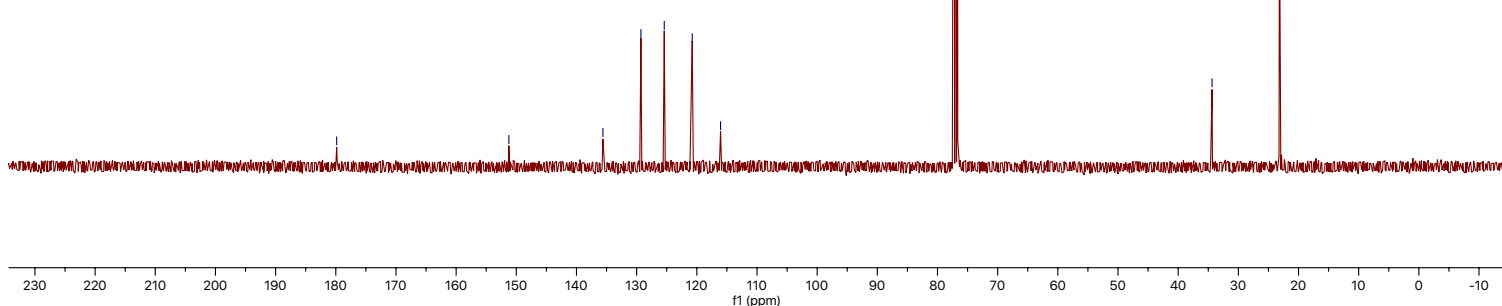


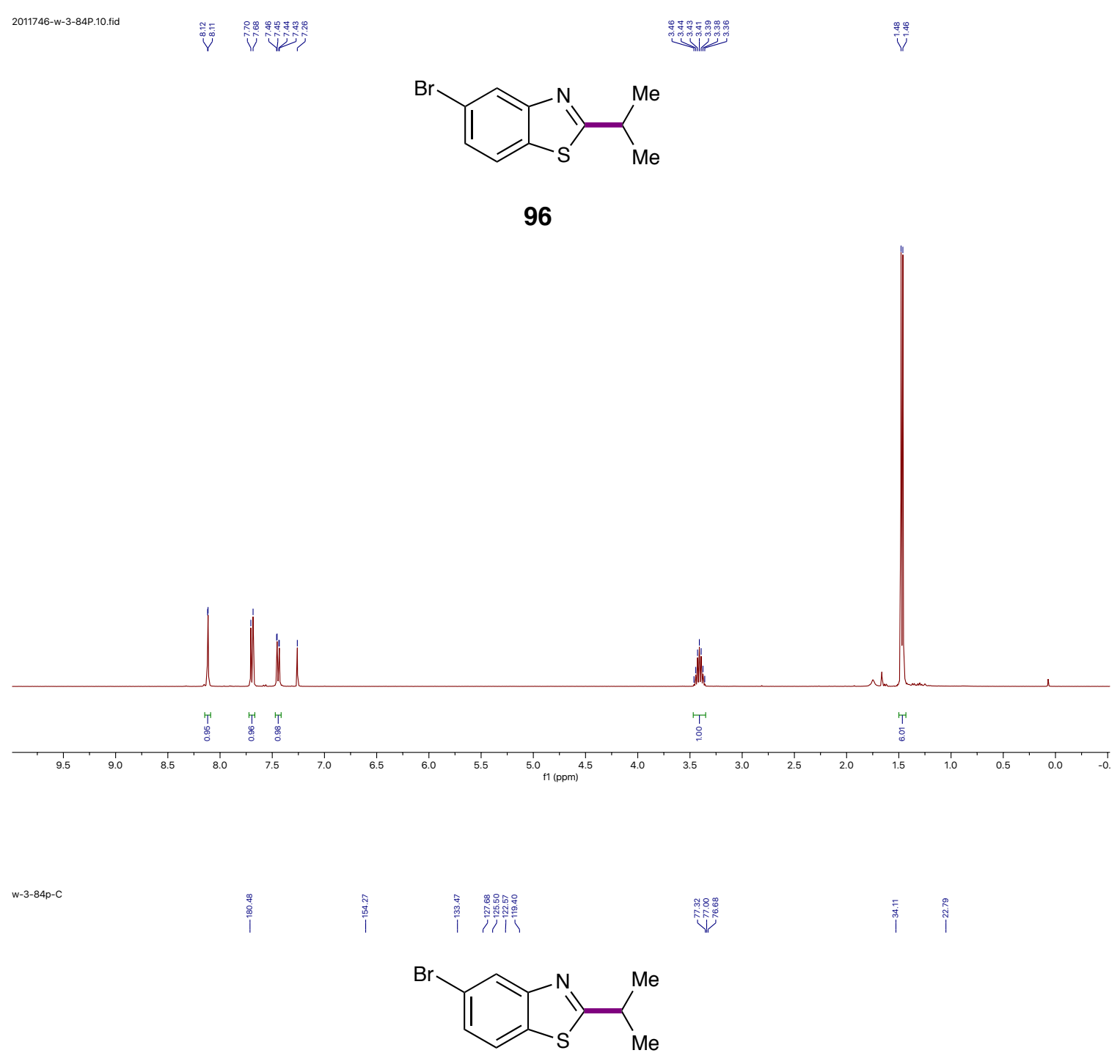

96
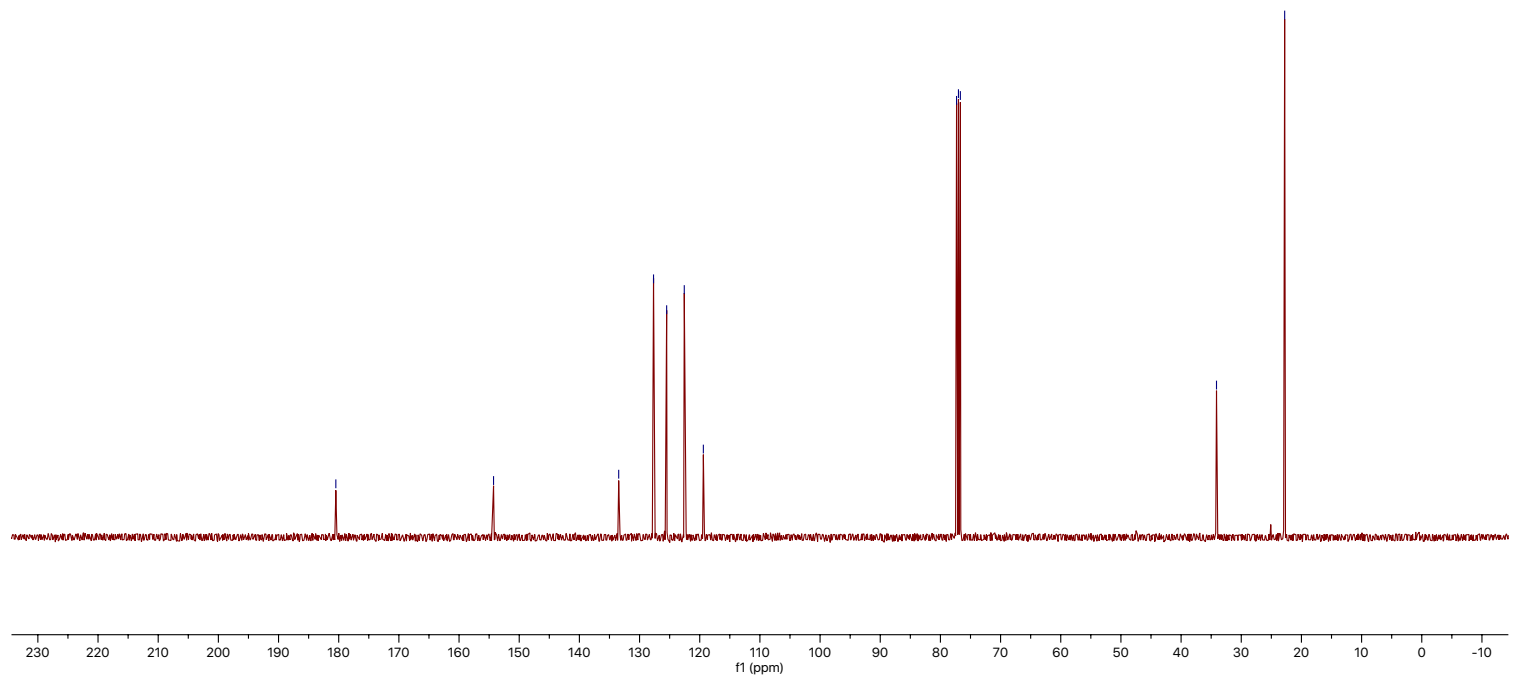


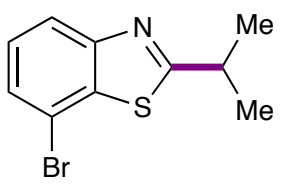

97

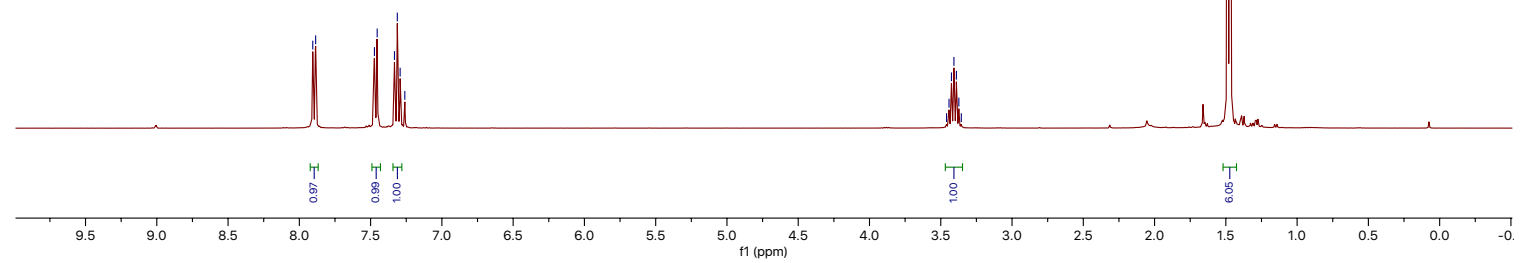

w-3-79p-C

|

的

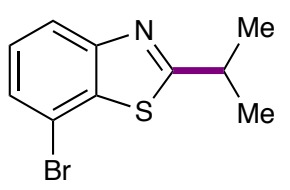

97

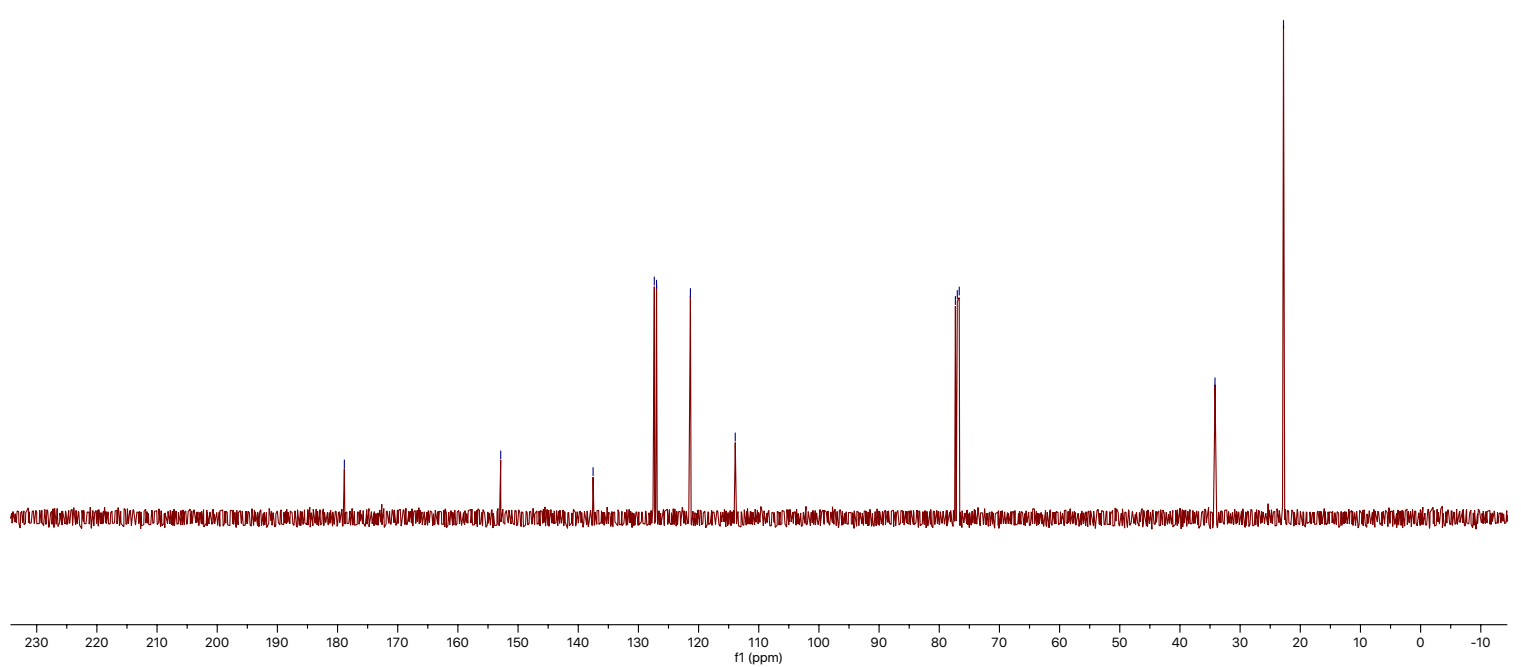


2011746-w-3-83P.10.fid

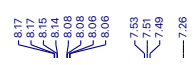

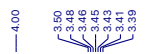

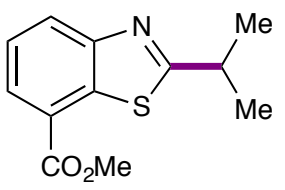

98

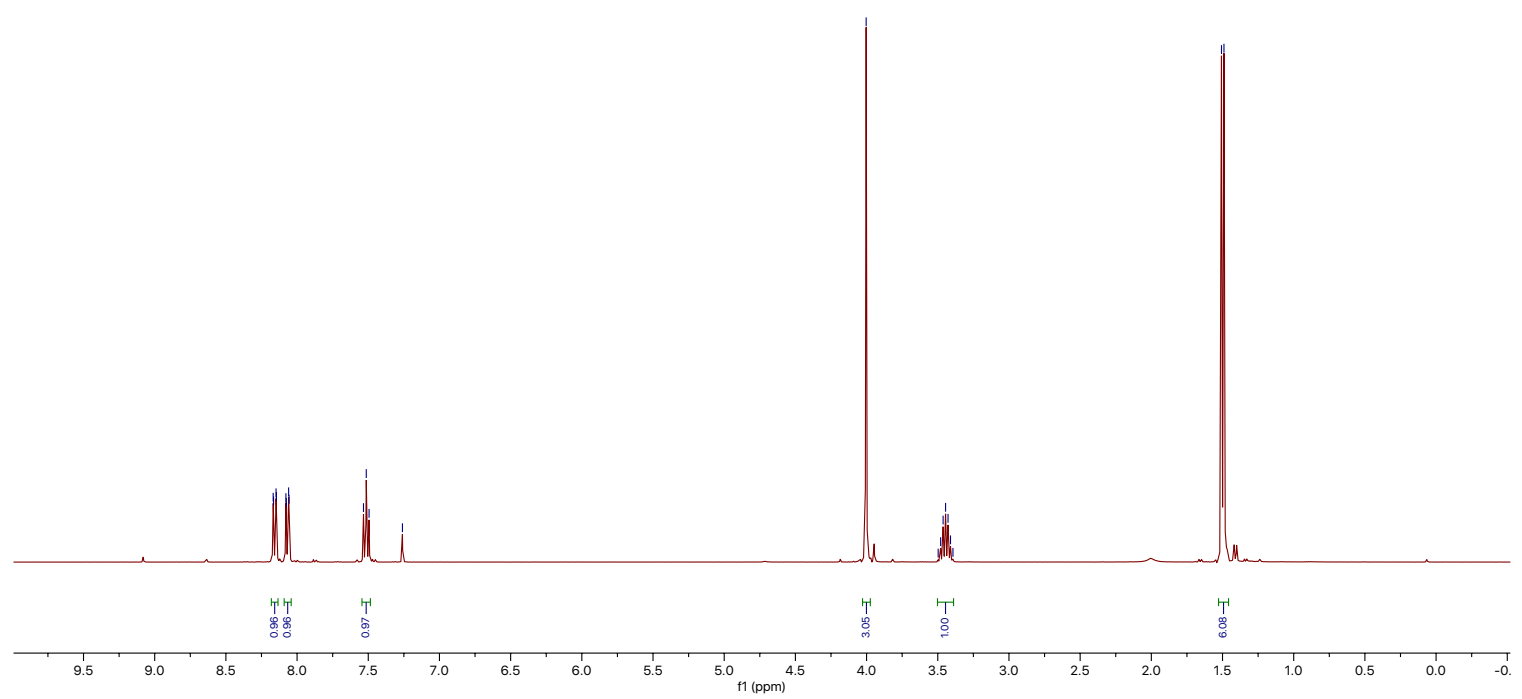

w-3-83p-c

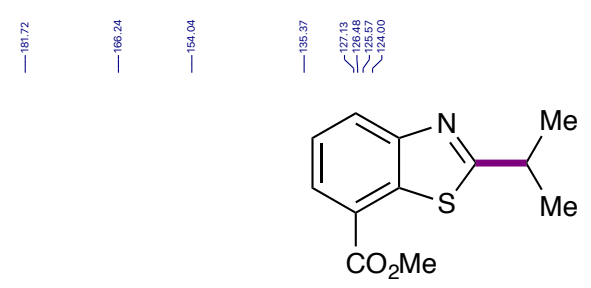

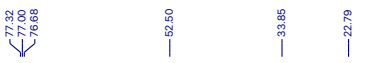

98

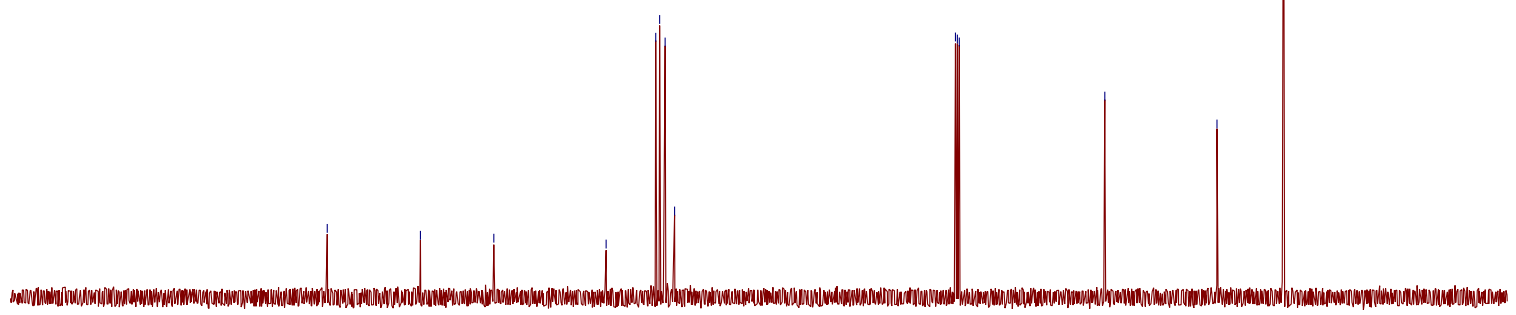

230 $\underset{11(\mathrm{ppm})}{11}$ 


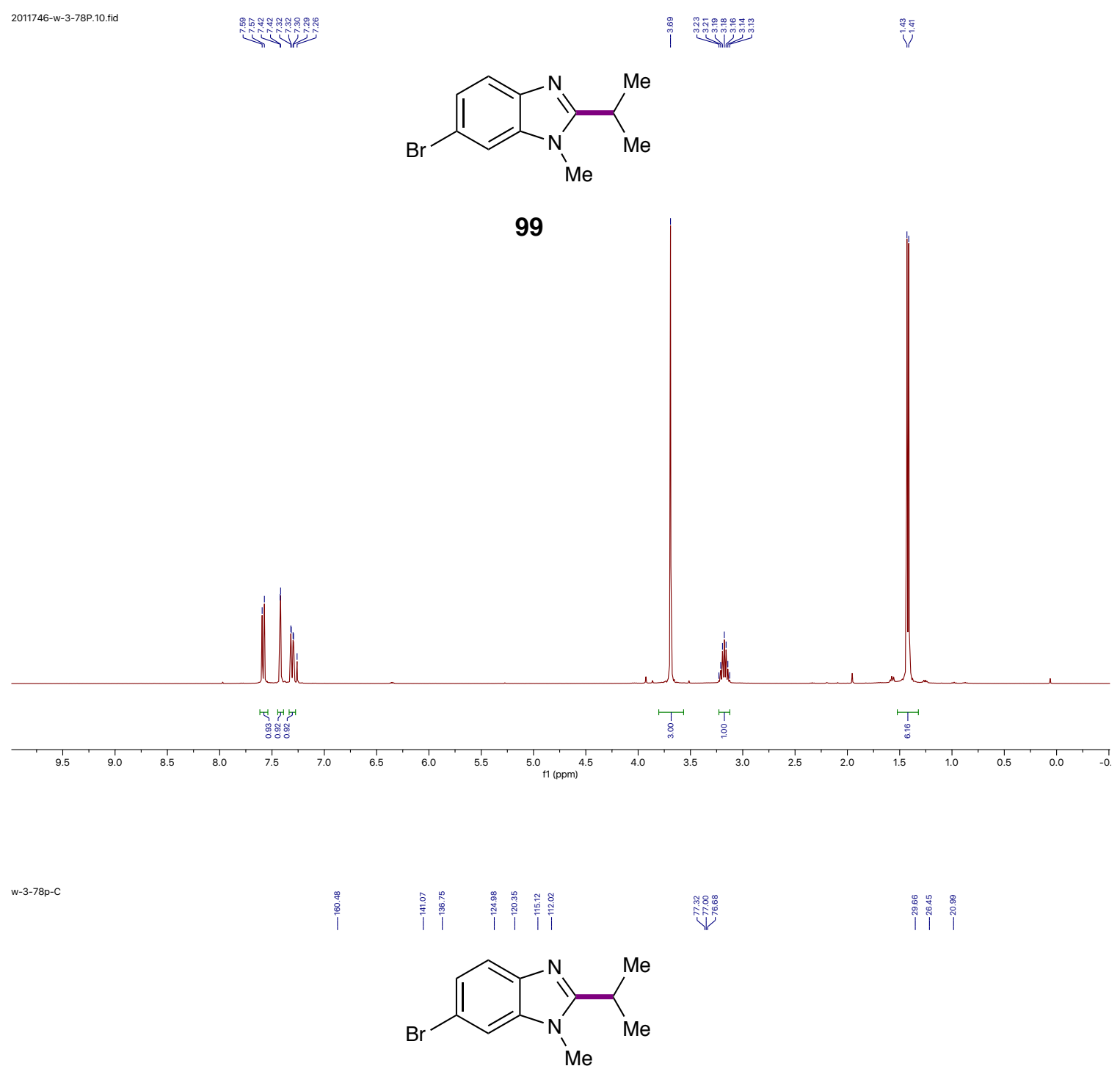

99

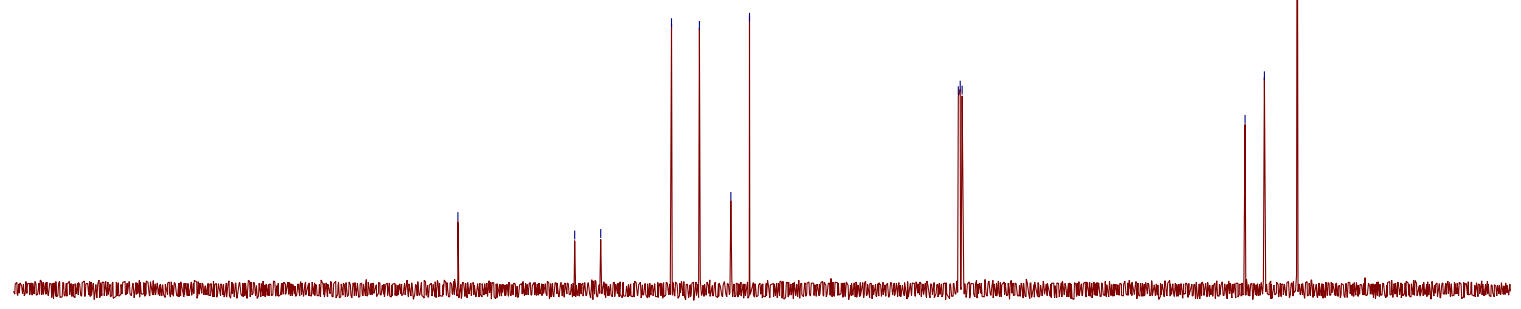

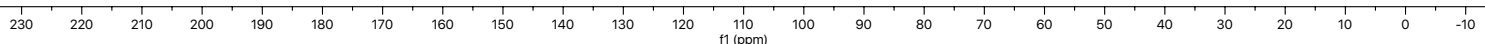



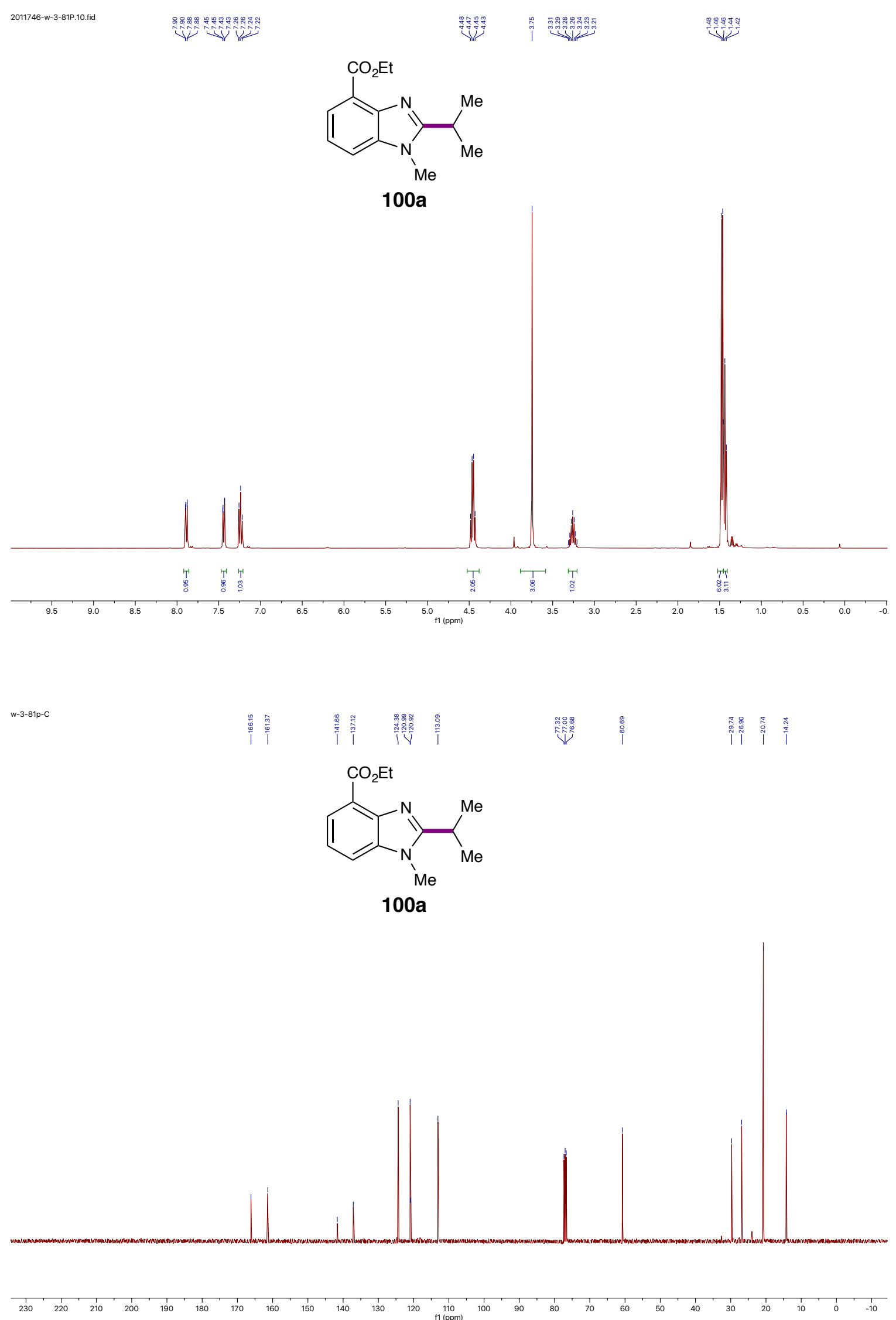

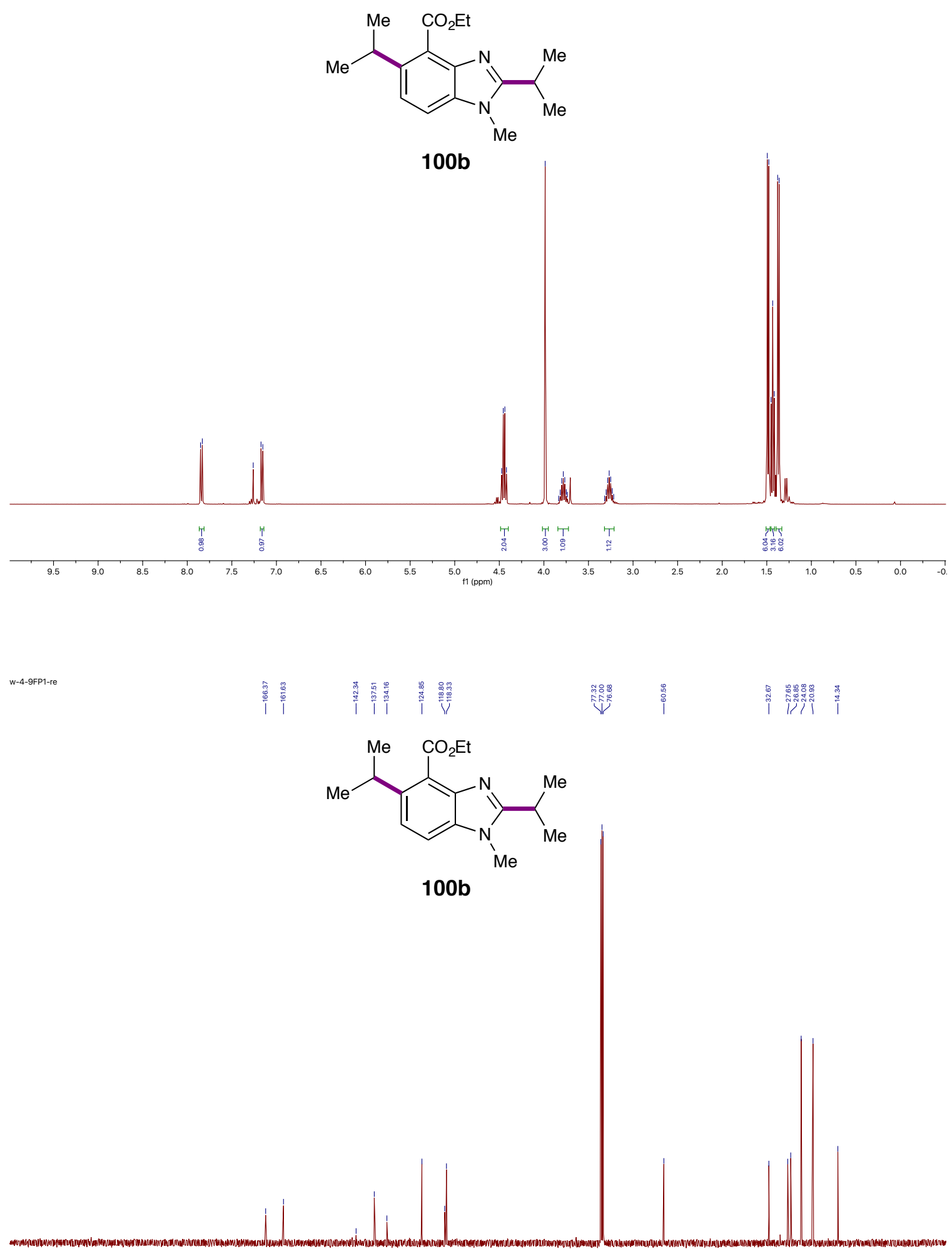

230

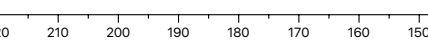

$120 \underset{\substack{110 \\ \mathrm{f} 1(\mathrm{ppm})}}{10} 10$ 


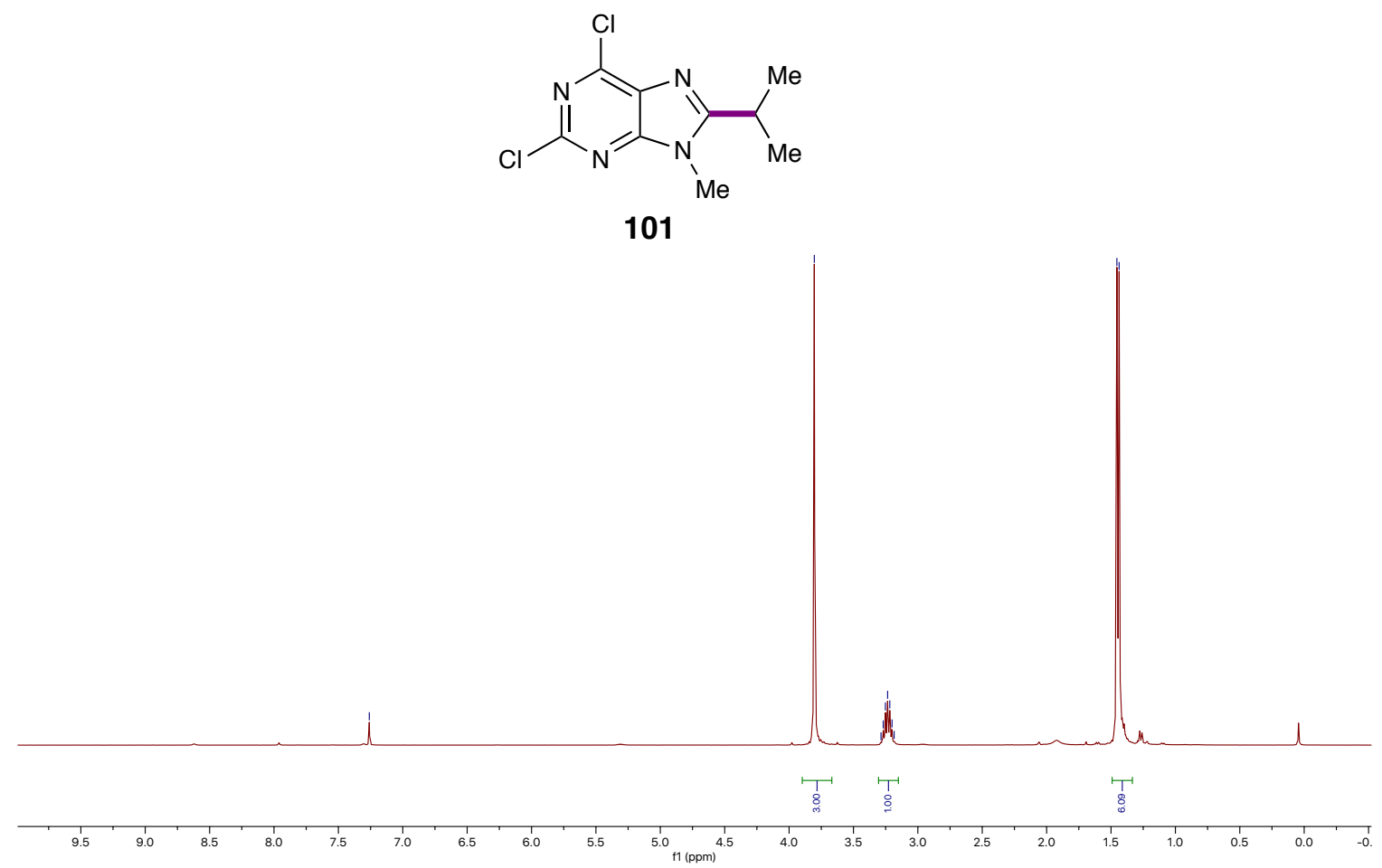

w-3-91BP-C

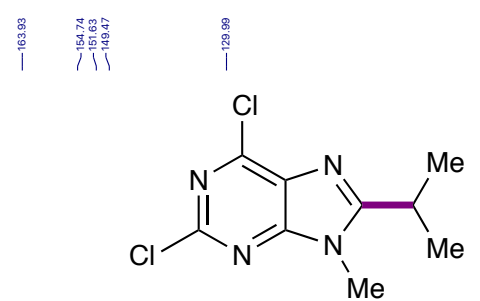

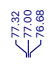

ำ

101

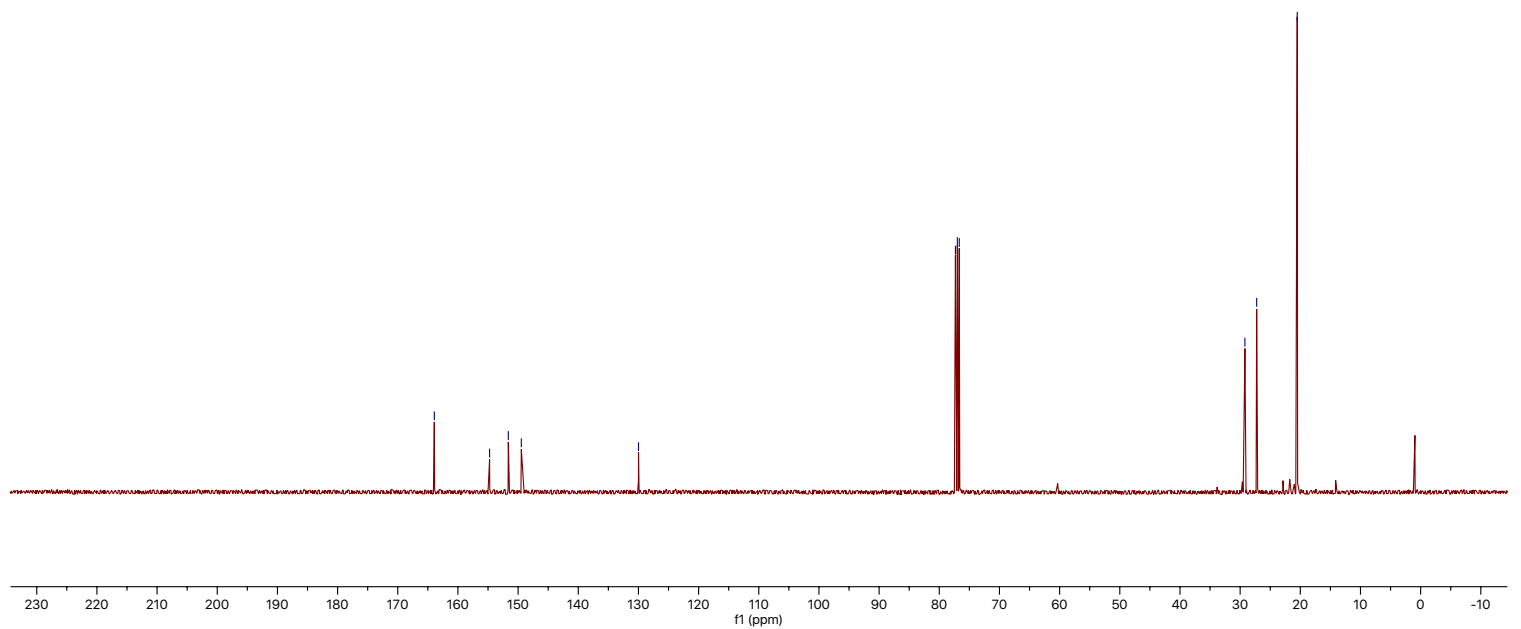

